\title{
Role of ASXL1 in Tumorigenesis and EMT in Breast Cancer
}

\author{
Doctoral Thesis
}

In partial fulfillment of the requirements for the degree

\author{
"Doctor rerum naturalium (Dr. rer. nat.)" \\ in the Molecular Medicine Study Program \\ at the Georg-August University Göttingen
}

submitted by

Anusha Budida

born in

Tuni, India

Göttingen, 2016 


\section{Members of the Thesis Committee:}

\section{Supervisor}

Prof. Dr. Steven A. Johnsen

Department of General, Visceral and Pediatric Surgery

University Medical Center Göttingen

Second member of the thesis committee

Prof. Dr. Michael Zeisberg

Department of Nephrology and Rheumatology

University Medical Center Göttingen

Third member of the thesis committee

Prof. Dr. med. Claudia Binder

Department of Haematology and Oncology

University Medical Center Göttingen

\section{Date of Disputation:}




\section{AFFIDAVIT}

Here I declare that my doctoral thesis entitled "Role of ASXL1 in Tumorigenesis and EMT in Breast Cancer" has been written independently with no other sources and aids than quoted.

Anusha Budida

Göttingen, November 2016 


\section{Table of contents}

Acknowledgements

Abbreviations iii

List of figures viii

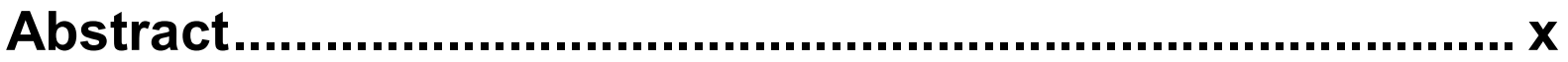

1. Introduction ...................................................................... 1

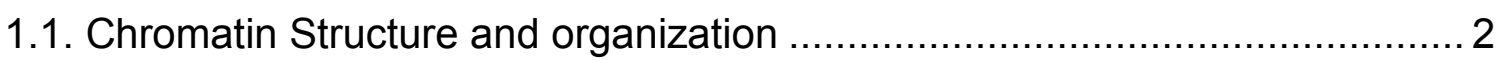

Figure. 1: The structure of the nucleosome. ........................ 3

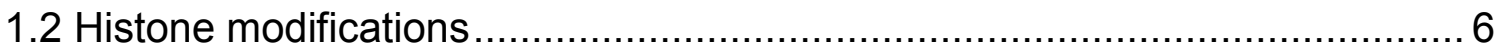

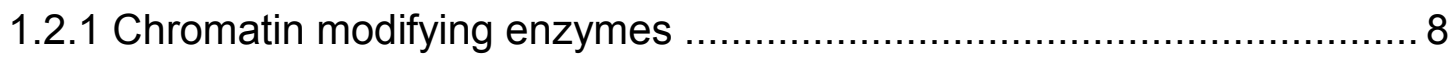

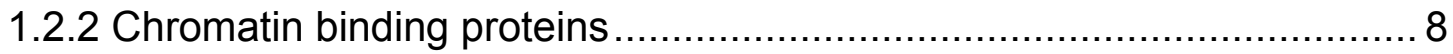

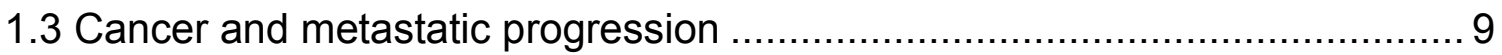

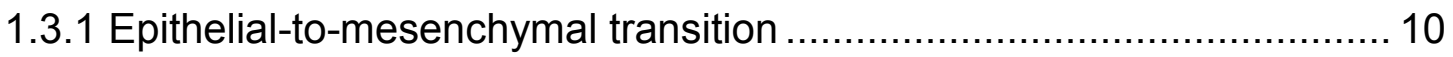

1.3.2 Mesenchymal-to-Epithelial transition .................................................. 11

1.3.3 Cancer stem cells and metastasis ............................................... 12

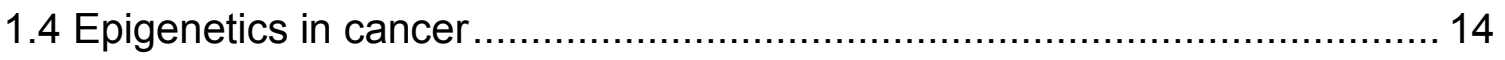

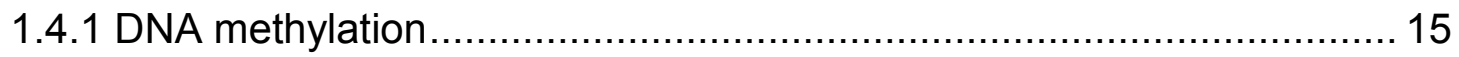

1.4.2 Histone modifications and their functional consequences ................... 16

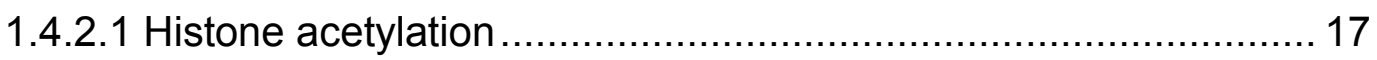

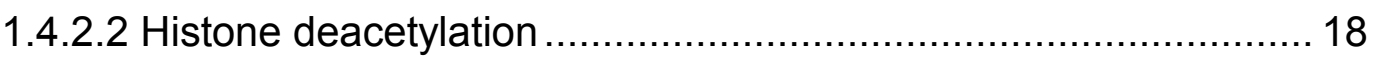

1.4.2.3 Histone methylation.......................................................... 18

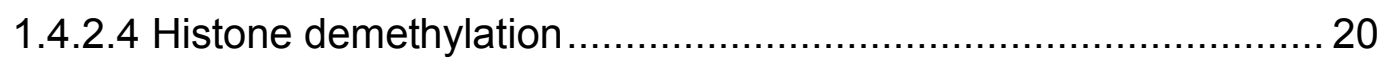

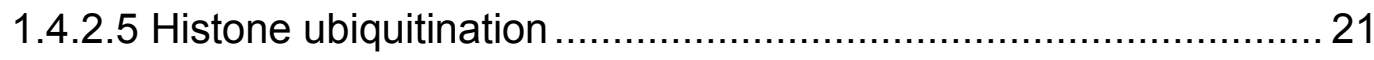

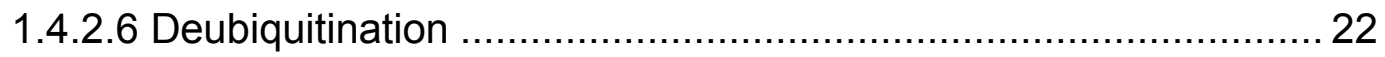

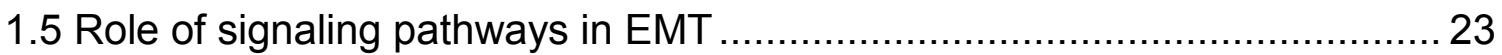




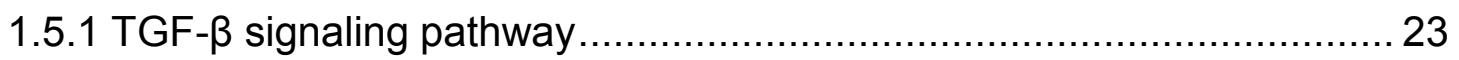

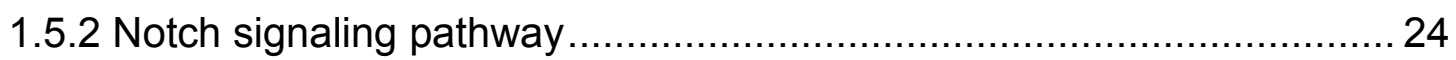

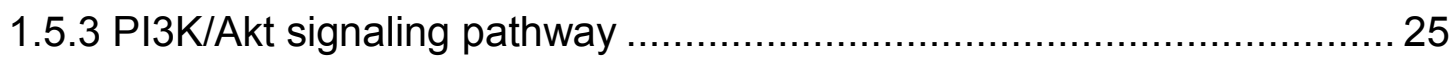

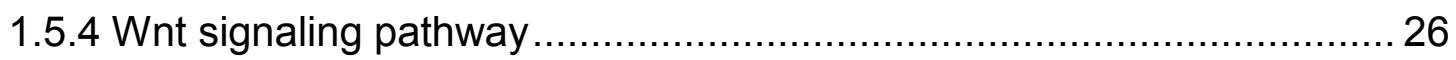

1.6 Additional Sex Comb-Like (ASXL), transcription regulator...........................2 27

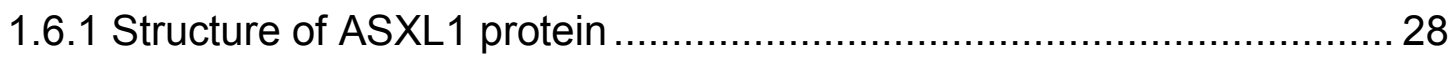

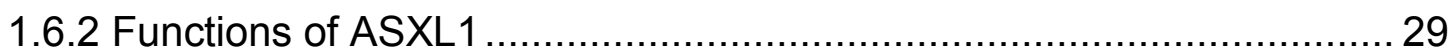

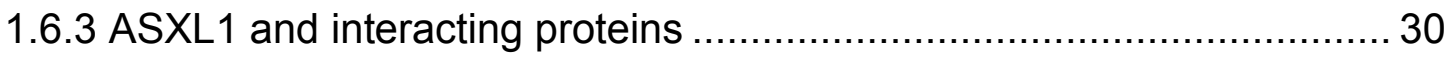

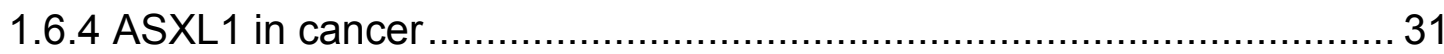

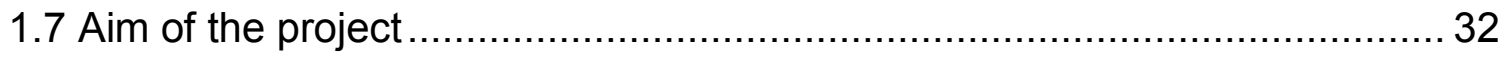

2. Materials ................................................................. 34

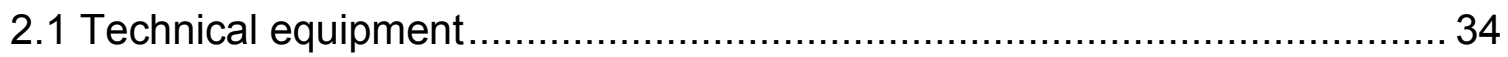

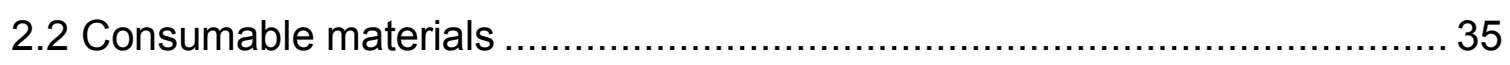

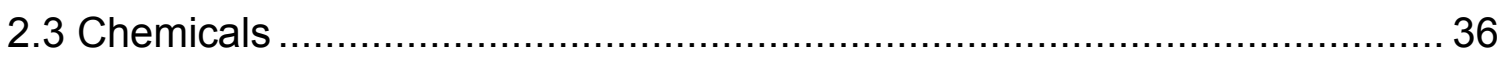

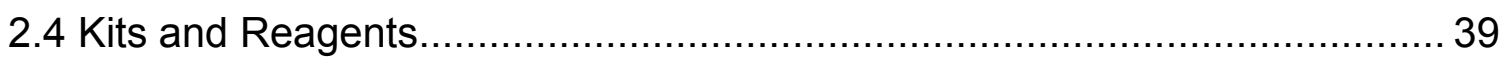

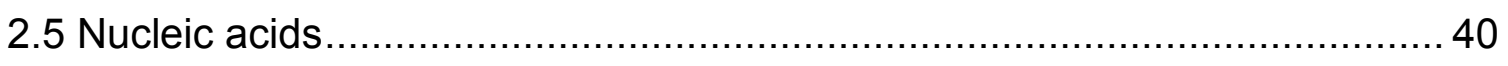

2.5.1 Vectors and expression constructs ................................................. 40

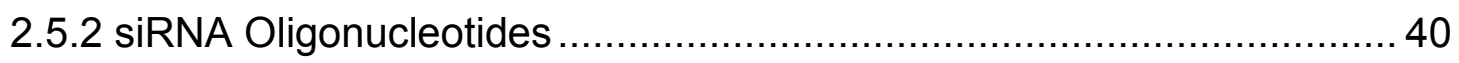

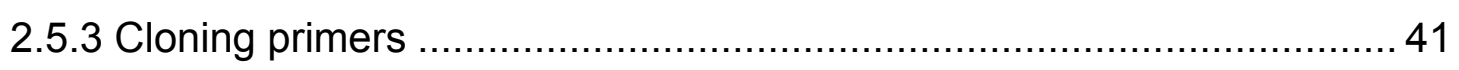

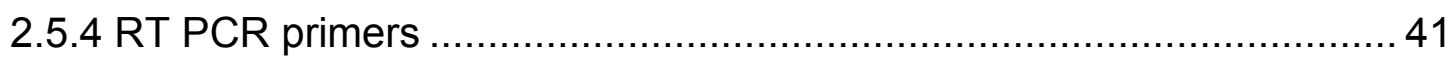

2.5.4.1 Quantitative PCR primers (qPCR) ........................................ 41

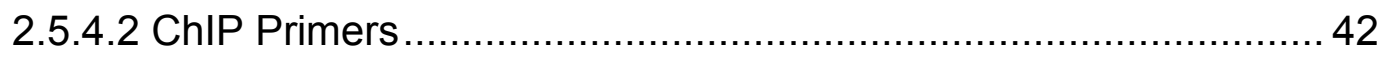

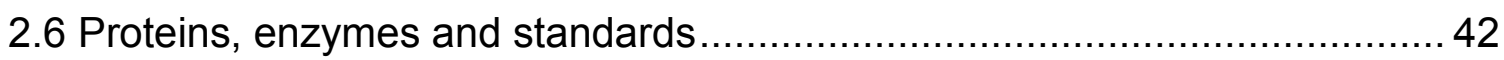

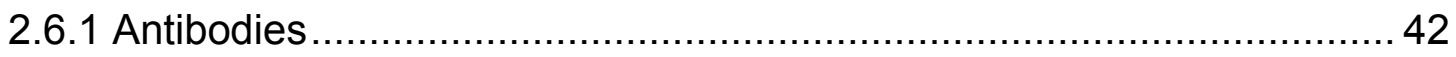

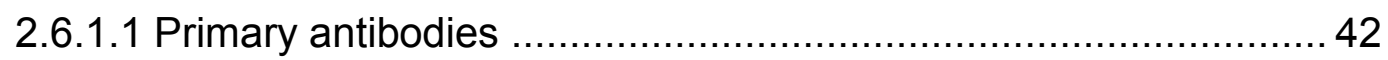

2.6.1.2 Secondary antibodies......................................................... 43 


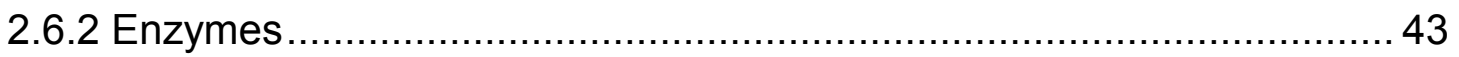

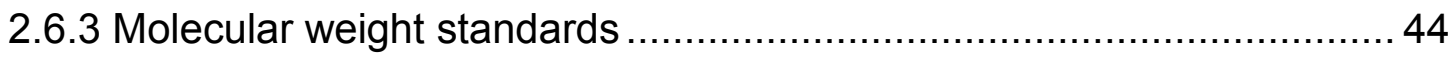

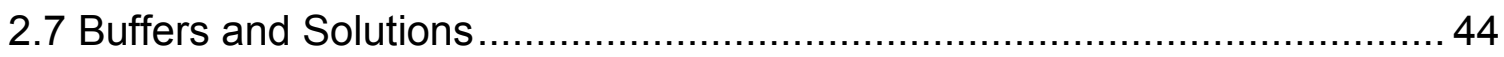

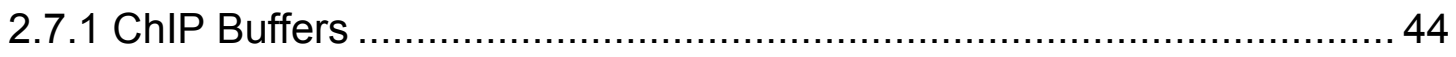

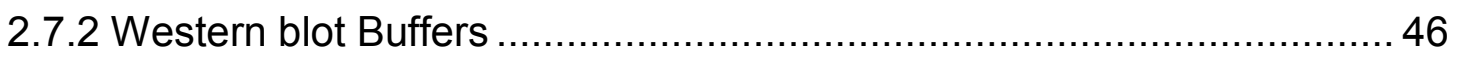

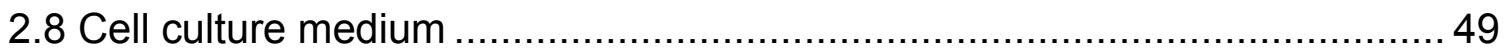

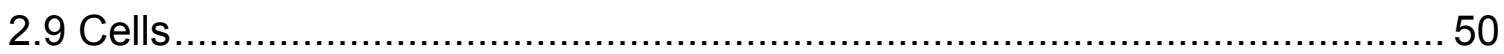

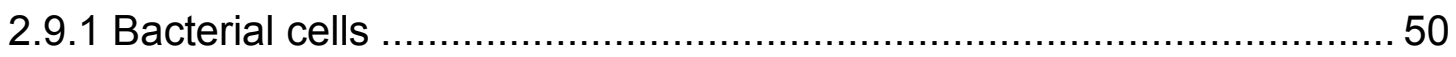

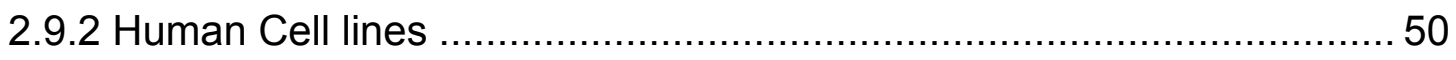

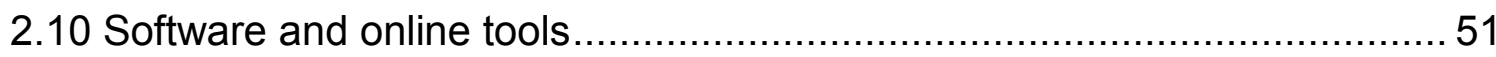

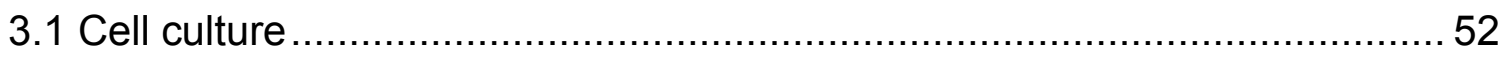

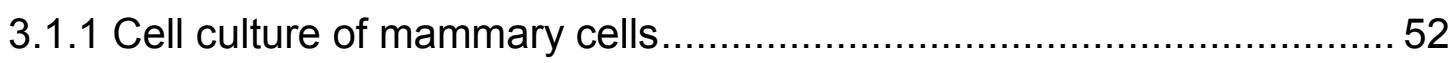

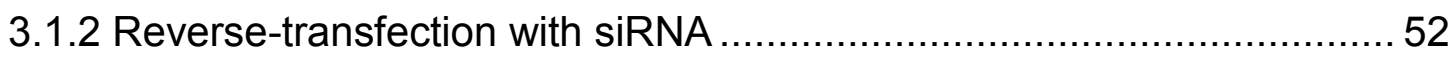

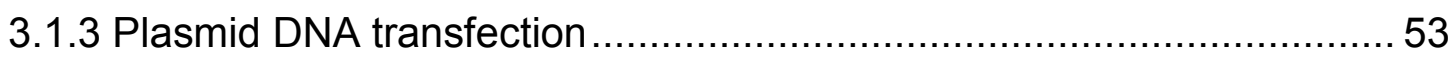

3.1.4 Stable transfection with plasmid DNA ............................................... 53

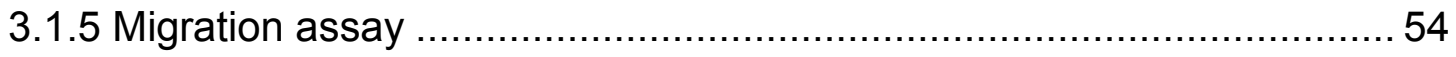

3.1.6 Mammosphere formation assay .................................................... 54

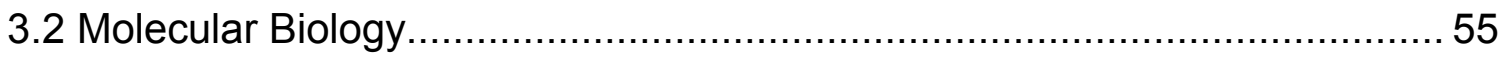

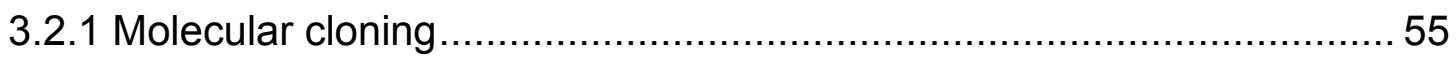

3.2.1.1 Polymerase chain reaction (Scott, Korfi et al.) ……................... 55

3.2.1.2 Restriction enzyme digestion ............................................... 56

3.2.1.3 Agarose gel purification of DNA fragments ............................... 57

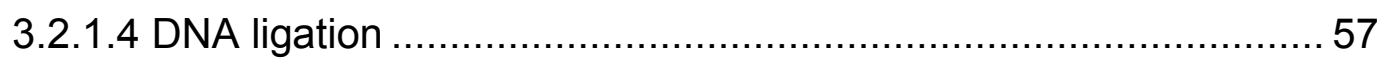

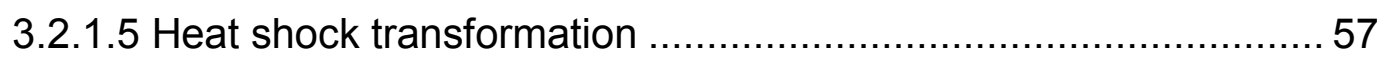

3.2.1.6 Screening of recombinant clones and plasmid isolation .............. 58

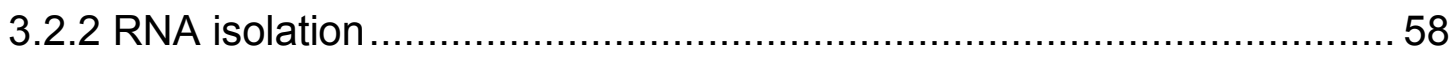




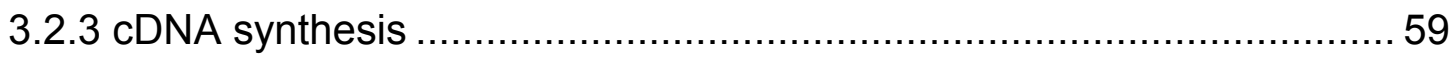

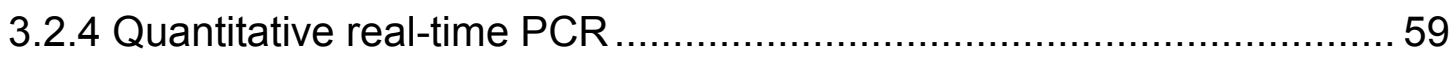

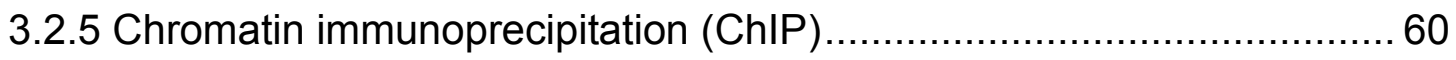

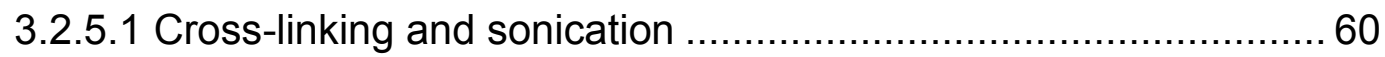

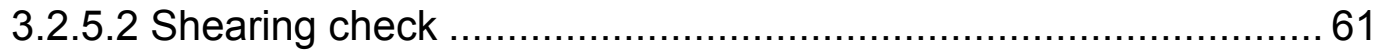

3.2.5.3 Pre-clearing and immunoprecipitation ...................................... 61

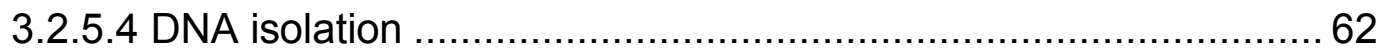

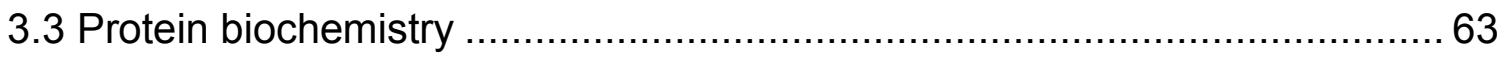

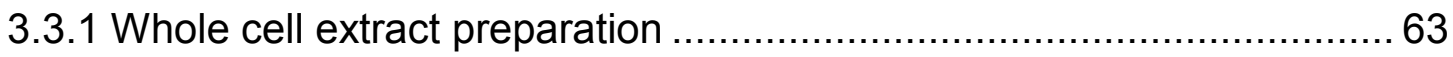

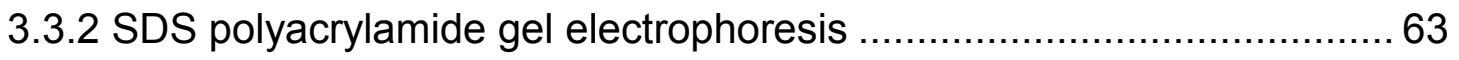

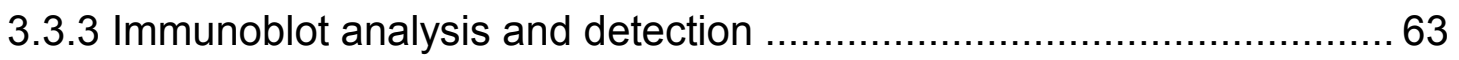

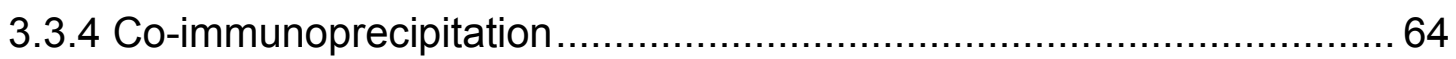

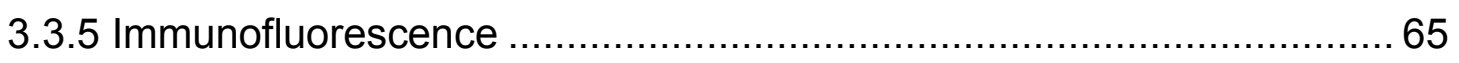

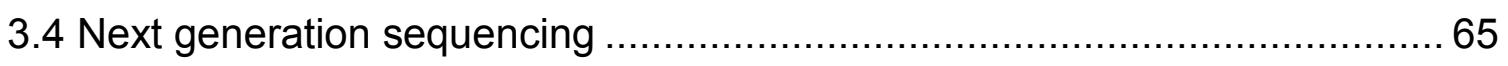

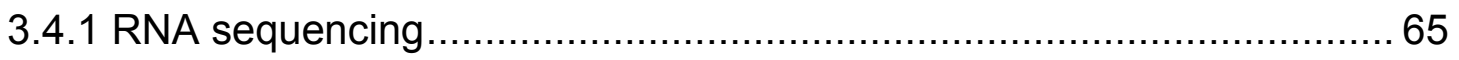

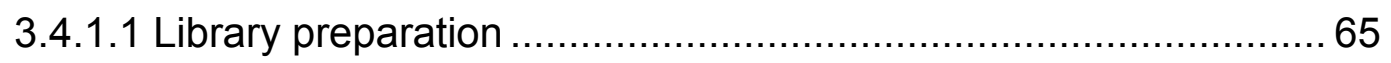

3.4.2 Chromatin immunoprecipitation sequencing (ChIP-seq) .....................66

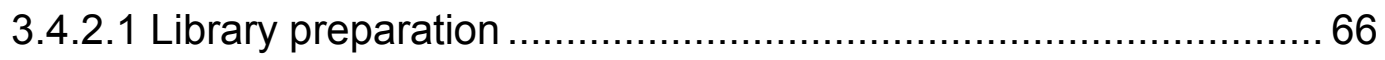

3.5 Bioinformatic analysis of RNA and ChIP sequencing data.........................67

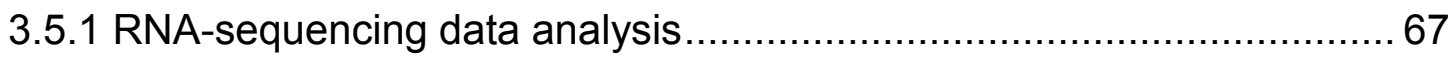

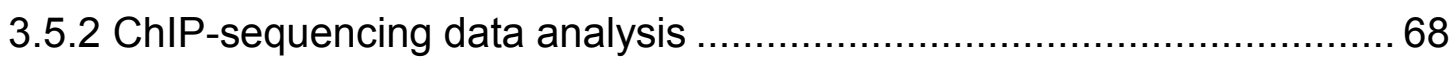

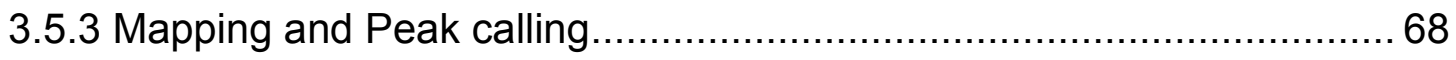

3.5.4 Normalization and visualization of ChIP- sequencing data .................. 69

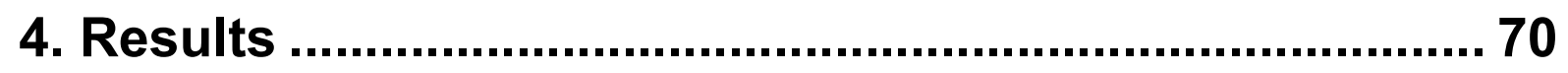

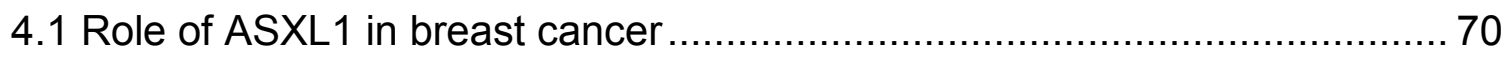


4.1.1 Expression of ASXL1 is downregulated in breast cancer ................... 70

4.1.2 ASXL1 expression and survival analysis ......................................... 71

4.2 ASXL1 and epithelial-to-mesenchymal transition.....................................72

4.2.1 ASXL1 downregulation perturbs the expression of EMT pathway genes

4.2.2 Loss of ASXL1 enhances EMT phenotype ....................................... 76

4.2.3 ASXL1 depletion enhances migratory properties.................................. 81

4.2.4 Loss of ASXL1 induces stem cell characteristics................................ 82

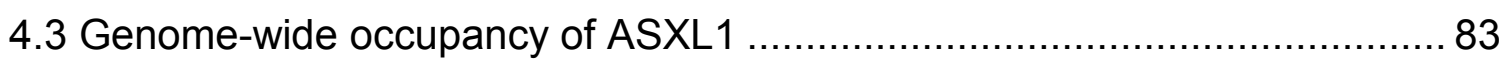

4.3.1 Enrichment of ASXL1 on promoter regions ……............................... 84

4.3.2 ASXL1 occupancy on individual genomic regions .............................. 86

4.4 ASXL1 occupancy correlate with gene expression ................................... 88

4.5 Generation of ASXL1 expressing stable cell line ...................................... 89

4.5.1 Ectopic expression of ASXL1 reverses EMT phenotype ..................... 89

4.5.2 ASXL1 expression reduces migratory properties ............................... 92

4.5.3 ASXL1 expression reduces stem cell characteristics ........................... 93

4.6 ASXL1 occupancy increased with ectopic ASXL1 expression ..................... 94

4.7.1 ASXL1 regulates epithelial gene expression by promoting the

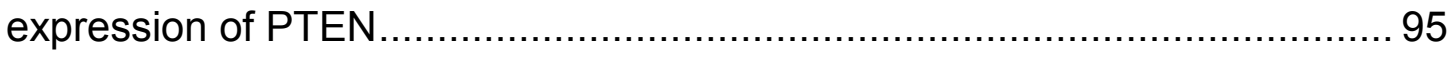

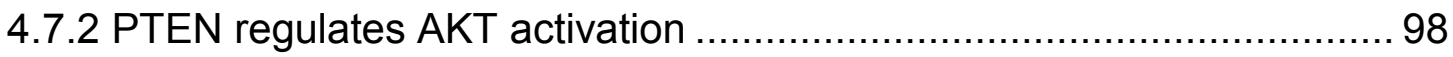

4.8 Depletion of ASXL1 regulates global levels of epigenomes ........................98

4.9.1 ASXL1 enhances the gene expression by epigenetic regulation ........ 100 4.9.2 ASXL1 depletion leads to decreased H3K4me3 on ASXL1 bound genes

4.9.3 ASXL1 cooperates with SET1 methylase complex to epigenetically

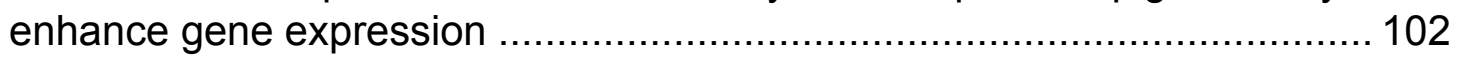

4.9.4 Depletion of ASXL1 leads to decreased expression of SET1 complex members 
5. Discussion 105

5.1 The role of ASXL1 as a potential tumor suppressor 106

5.2 ASXL1 downregulation perturbs the global gene expression. 107

5.3 The role of ASXL1 in cancer metastasis 108

5.3.1 The role of $A S X L 1$ in regulating EMT 108

5.3.2 ASXL1 expression regulates cancer stem cell pool 110

5.4 Genome-wide distribution of ASXL1 111

5.5 ASXL1 regulates EMT by targeting PTEN 112

5.6 ASXL1 and post-translational modifications 113

5.7 Loss of ASXL1 is associated with occupancy of H3K4me3 114

5.8 ASXL1 physically interacts with BAP1 and SET1 methyltransferase complex members

5.9 ASXL1 regulates expression of SET1 complex members. 


\section{Acknowledgements}

First of all, I would like to express my sincere gratitude to my mentor and my supervisor Prof. Dr. Steven A. Johnsen, for giving me an opportunity to work with him. I am extremely grateful to him for all the continuous support, freedom and encouragement he has extended starting from my application for DAAD fellowship and through the course of my studentship. I always admire his perseverance and passion towards science. Moreover, I would like to thank him for the friendly atmosphere that he created in our group and for being available for help at all times.

It is my pleasure to extend my sincere thanks to Prof. Hans K. Will for great help, discussion and valuable suggestions in the process of my thesis preparations. He is a great human being and a wonderful advisor.

I thank my thesis committee members Prof. Dr. Michael Zeisberg and Prof. Dr. Claudia Binder for their support and comments and thoughtful discussions.

I would like to thank German Academic Exchange Service (DAAD) for the financial support during my course of Ph.D. and it would not have possible to start my Ph.D. without their support.

I would like to thank Prof. M.R.S. Rao (JNCASR, India) for introducing me to the field of chromatin and epigenetics and for choosing me as one of the members of chromatin biology group. I express my thanks to the members of Prof. M.R.S. Rao's lab, especially Dr. Surbhi Dhar, for their help and making my work experience in the lab memorable.

I would like to thank all the current and former members of the AG Johnsen for a wonderful working atmosphere and also for their cooperation. 
I would like to acknowledge all my teachers, starting from my school to my post-graduation, for their motivation throughout the learning process. It is my great pleasure to thank my M.Sc. thesis supervisor Prof. Nihar Ranjan Jana (NBRC, India) for his encouragement and for motivating me to do research. I am also thankful to his group members, especially Dr. Sudheendra Rao, for their support and introducing me to molecular techniques.

It is my pleasure to express my sincere love to my mother, Satyaveni Thota. I consider myself lucky to have her in my life as she showered me with her care, love and affection and has given me all the freedom I wanted to carefully choose my life by myself. She encouraged me to pursue my studies even in the most difficult situations of our lives. I would like to thank my sister Haritha, my best friend for lending her patient ears and shoulder to lean on at the times of difficulty and for the love and constant support and also my bother-in-law Somasekhar, for his support and making our lives easier. My special thanks to all my friends for their love and caring during all the situations. I thank the divine soul of my father for showing his blessings and for making everything possible.

A big thanks to my husband, Ramachandramouli, the person I am blessed with, in my life. I am extremely grateful to him for his unconditional love and support. His encouraging words and his affection often made my dull days bright. Thank you so much for every good thing that you brought in my life.

Finally, I thank the almighty, for his blessings and showing me the path even in difficult situations. 


\section{Abbreviations}

$\% \quad$ Percentage

$(\mathrm{NH} 4)_{2} \mathrm{SO}_{4} \quad$ Ammonium Sulphate

${ }^{\circ} \mathrm{C}$ degree Celsius / centigrade

$\mu \mathrm{g} \quad$ microgram

$\mu \mathrm{l} \quad$ microliter

$\mu \mathrm{M} \quad$ micromolar

APS Ammonium persulfate

ASXL Additional Sex Combs Like

bam Binary Version of sam files

BAP1 BRCA1 Associated Protein 1

BGP $\quad \beta$-glycerophosphate

BGS Bovine Growth Serum

bigwig Binary Version of wiggle files

bp base pair

BSA Bovine Serum Albumin

CDH1 E-cadherin

$\mathrm{CDH} 2 \quad \mathrm{~N}$-cadherin

cDNA Complementary DNA

Cfp1 CxxC finger protein 1

ChIP Chromatin Immunoprecipitation

ChIP-seq ChIP followed by high-throughput sequencing

$\mathrm{CO}_{2} \quad$ Carbon dioxide

CpG Cytosine phosphate Guanine

CSCs Cancer Stem Cells

DAPI 4', 6-diamidino-2-phenylindole

DEPC Diethylpyrocarbonate

DMEM Dulbecco modified eagle's Minimal Essential medium

DMSO Dimethyl sulfoxide

DNA Deoxyribonucleic acid 
DNMT DNA Methyltransferase

dNTP Deoxy ribonucleotide triphosphate

DTT Dithiothreitol

DUB Deubiquitinating enzyme

EDTA Ethylene Diamine Tetra Acetic acid

EGF Epidermal Growth Factor

EMT Epithelial-to-mesenchymal transition

EMT-TF EMT-transcription factor

EZH2 Enhancer of zest homolog 2

FBS Fetal Bovine Serum

bFGF basic Fibroblast Growth Factor

FN1 fibronectin

g gravity

GO Gene Ontology

h hour

$\mathrm{H} 1 \quad$ histone 1

H2A Histone 2A

H2Aub1 Monoubiquitinated histone 2A

H2B Histone 2B

H2Bub1 Monoubiquitinated histone 2B

H3 Histone 3

H3K27me3 Histone 3 trimethylated at position lysine 27

H3K4me3 Histone 3 trimethylated at position lysine 4

H4 Histone 4

HAT Histone acetyltransferase

HMT Histone methyltransferase

HNRNPK heterogeneous nuclear ribonucleoprotein $\mathrm{k}$

HRP Horse Radish Peroxidase

HSC70 Heat Shock $70 \mathrm{KDa}$ protein

IAA lodacetamide 


\begin{tabular}{|c|c|}
\hline IF & Immunofluorescence \\
\hline $\lg G$ & Immunoglobulin G \\
\hline IGV & Integrative Genomics Viewer \\
\hline $\mathrm{K}_{2} \mathrm{HPO}_{4}$ & Dipotassium phosphate \\
\hline $\mathrm{kb}$ & kilo base pairs \\
\hline $\mathrm{KCl}$ & Potassium Chloride \\
\hline $\mathrm{KDa}$ & Kilo Dalton \\
\hline $\mathrm{kg}$ & kilogram \\
\hline $\mathrm{KH}_{2} \mathrm{PO}_{4}$ & Monopotassium phosphate \\
\hline LB & Lysogeny Broth \\
\hline $\mathrm{LiCl}$ & Lithium Chloride \\
\hline LSD1 & Lysine-Specific Demethylase 1 \\
\hline $\mathrm{m}$ & milli $\left(10^{-3}\right)$ \\
\hline M & molar, mol/L \\
\hline MACS & Model-based Analysis of ChIP-seq \\
\hline MCF10A & Michigan Cancer Foundation-10A \\
\hline MCF12A & Michigan Cancer Foundation-10A \\
\hline MET & Mesenchymal-to-epithelial transition \\
\hline $\mathrm{mg}$ & milligram \\
\hline $\mathrm{MgCl}_{2}$ & Magnesium Chloride \\
\hline $\min$ & minute \\
\hline $\mathrm{ml}$ & milliliter \\
\hline $\mathrm{mM}$ & millimolar \\
\hline mRNA & messenger RNA \\
\hline $\mathrm{n}$ & nano $\left(10^{-9}\right)$ \\
\hline n.s. & non-significant \\
\hline $\mathrm{Na}_{3} \mathrm{VO}_{4}$ & Sodium orthovanadate \\
\hline $\mathrm{NaCl}$ & Sodium Chloride \\
\hline NAF & Sodium Fluoride \\
\hline $\mathrm{NaH}_{2} \mathrm{PO}_{4}$ & Sodium dihydrogen phosphate \\
\hline
\end{tabular}




\begin{tabular}{|c|c|}
\hline $\mathrm{NaN}_{3}$ & Sodium Azide \\
\hline $\mathrm{NCBI}$ & national center for biotechnology information \\
\hline NEM & $\mathrm{N}$-ethylmaleimide \\
\hline NES & Normalized Enrichment Score \\
\hline $\mathrm{NiCl}_{2}$ & Nickel Chloride \\
\hline $\mathrm{nm}$ & nanometer \\
\hline NP-40 & Nonidet P40 \\
\hline p300 & e1a binding protein $\mathrm{p} 300$ \\
\hline pAdj & Adjacent $p$ value \\
\hline PBS & phosphate buffered saline \\
\hline PBST & phosphate Buffered Saline with Tween-20 \\
\hline PCR & Polymerase Chain Reaction \\
\hline $\mathrm{pH}$ & potential of hydrogen \\
\hline PHD & plant homeodomain \\
\hline $\mathrm{PI} 3 \mathrm{~K} / \mathrm{Akt}$ & Phosphotidylinositol-3-Kinase and Protein Kinase B \\
\hline PRC1 & Polycomb repressive Complex 1 \\
\hline PRC2 & Polycomb repressive Complex 2 \\
\hline PTEN & phosphatase and tensin homolog \\
\hline qRT-PCR & Quantitative real-time PCR \\
\hline RIPA & Radio-Immunoprecipitation Assay buffer \\
\hline RNA-seq & Sequencing of rt-transcribed RNA \\
\hline RNF20 & Ring Finger Protein 20 \\
\hline RNF40 & Ring Finger Protein 40 \\
\hline RT & Room Temperature \\
\hline RT-PCR & Reverse transcription PCR \\
\hline sam & Sequence Alignment map \\
\hline SDS & Sodium dodecyl Sulfate \\
\hline SDS-PAGE & SDS- polyacrylamide Gel Electrophoresis \\
\hline sec & second \\
\hline siControl & negative control sirna \\
\hline
\end{tabular}




\begin{tabular}{ll} 
SiRNA & small interfering RNA \\
TAE & Tris-acetate-EDTA \\
Taq & Thermus aquaticus \\
TBST & Tris-buffered saline and tween 20 \\
TE & Tris-EDTA \\
TEMED & Tetra methyl ethylene diamine \\
TGF- $\beta$ & transforming growth factor beta \\
TJP3 & Tight junction protein 3 \\
TR & Transcribed Region \\
Tris & Tris(hydroxymethyl)aminomethane \\
TSS & Transcription Start Site \\
U & unit (enzyme activity) \\
ub & ubiquitin \\
V & voltage \\
v/v & volume per volume \\
VIM & Vimentin \\
vs. & versus \\
w/v & weight per volume \\
Wdr & WD repeat domain \\
ZEB1 & Zinc finger E-box binding homeobox 1 \\
ZO1 & Zonula occludes1 \\
\hline
\end{tabular}




\section{List of figures}

Figure. 1: The structure of the nucleosome............................................. 3

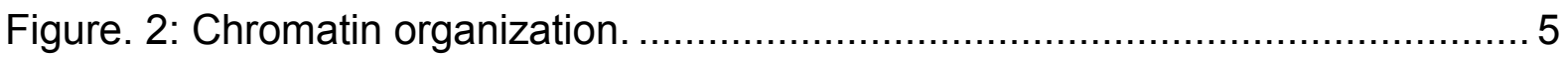

Figure. 3: Main post-translational histone modifications................................ 7

Figure. 4: EMT and MET in carcinogenesis and tumor metastasis. ..................... 13

Figure. 5: Schematic representation of ASXL1 protein structure ....................... 29

Figure. 6: ASXL1 gene expression in breast carcinoma................................ 71

Figure. 7: ASXL1 expression level predicts the disease outcome....................... 72

Figure. 8: ASXL1 regulate EMT and metastasis properties............................ 75

Figure. 9: Depletion of ASXL1 induces EMT phenotype. ............................. 78

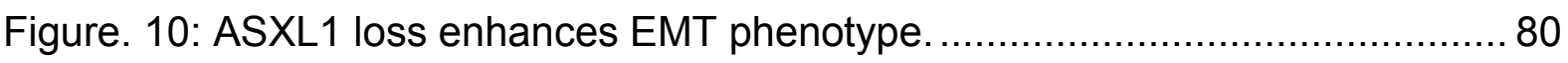

Figure. 11: Loss of ASXL1 promotes migratory potential. ............................... 82

Figure. 12: ASXL1 depletion induces stem-cell properties.............................. 83

Figure. 13: ASXL1 enrichment in the genome.......................................... 86

Figure. 14: ASXL1 enrichment on the promoters of single genes. ...................... 87

Figure. 15: ASXL1 directly regulates gene expression................................ 89

Figure. 16: Expression of ASXL1 induces MET phenotype........................... 91

Figure. 17: ASXL1 expression reverses EMT phenotype............................... 92

Figure. 18: Expression of ASXL1 suppresses migratory potential.......................93

Figure. 19: Expression of ASXL1 depletes stem-cell properties ......................... 94

Figure. 20: Enrichment of ASXL1 increased with ectopic ASXL1 expression. .........95 
Figure. 21: PTEN expression and its effect on EMT markers. 96

Figure. 22: ASXL1 loss enhances EMT phenotype. 97

Figure. 23: PTEN depletion enhances AKT activation. 98

Figure. 24: ASXL1 depletion regulates histone methylation and monoubiquitination levels.

Figure. 25: H3K4me3 enrichment correlates with gene expression. 100

Figure. 26: H3K4me3 enrichment on the promoters of ASXL1 target genes. 102

Figure. 27: ASXL1 and BAP1 associate with SET1 complex members. 103

Figure. 28: ASXL1 depletion regulates expression of SET1 complex members. ... 104 


\section{Abstract}

Breast cancer is one of the types of cancer that occurs in women frequently and most of the cancer deaths are due to metastasis. During cancer metastasis, the tumor epithelial cells lose their cell-cell contacts, acquire mesenchymal, migratory and invasive characteristics through epithelial-to-mesenchymal transition (EMT). Although many studies shed light on the mutations of ASXL family members in several cancers but the mechanism of action and the regulation remained poorly understood. ASXL1 is considered as a novel type of tumor suppressor in myeloid malignancies, which acts through the regulation of cell proliferation.

In this study, we showed that the depletion of ASXL1 in normal mammary epithelial cells leads to the loss of epithelial characteristics and gain stem cell-like, migratory and metastatic properties, which is characterized by the increased expression of the mesenchymal markers and decreased expression of the epithelial markers. Global RNA-seq transcriptome analysis revealed an enrichment of gene signatures associated with a mammary stem cell phenotype and EMT pathways upon ASXL1 knockdown. We also examined the genome-wide binding of ASXL1 via ChIP sequencing and identified a preferential occupancy of ASXL1 near transcriptional start sites of a subset of ASXL1 target genes. From our results, we found PTEN as a target gene and the depletion of PTEN promotes EMT phenotype.

Depletion of ASXL1 decreases the enrichment of $\mathrm{H} 3 \mathrm{~K} 4 \mathrm{me} 3$ at the promoters of target genes. ASXL1 is physically interacting with methyltransferase SET1 complex members (Cfp1 and Wdr82) and it might be required for the maintenance of the cellular expression of SET1 complex members (Cpf1 and Wdr82). Taken together, our results suggest that ASXL1 is a tumor suppressor and negatively regulates EMT. 
ASXL1 might promote transcription of a subset of ASXL1 affected genes either by directly binding to the target genes or through the active mark, H3K4me3 on the promoters. Further studies may provide additional insight which may be useful for developing new therapeutic targets for preventing or treating breast cancer metastasis. 


\section{Introduction}

Breast cancer is one of the most common among all cancers in women and the second most frequent cause of cancer-related deaths in females worldwide. And more importantly, around $90 \%$ of them are caused by invasion and metastasis of the tumor cells. It also occurs, but rarely, in men. It accounts $23 \%$ of total cancer patients and $14 \%$ of all cancer deaths (Jemal, Bray et al. 2011). The breast is made up of glands which contain lobules and ducts. The most common type of breast cancer is ductal carcinoma.

Cancer development generally begins with a series of molecular events that alter the properties of normal cells, which leads to abnormal cell growth and division. Normal cells consist of a sophisticated system to check and control cell overgrowth, which is dysregulated in the cancerous cells making them to proliferate in an uncontrolled and indefinite manner. The loss of growth control is a result of the accumulation of genetic abnormalities like mutations, deletions, translocation or amplification. The mutated or malfunctioning genes can be grouped into three classes, such as oncogenes, tumor suppressors and DNA repair genes. Oncogenes are a mutated version of proto-oncogenes which normally enhance cell division and prevent cell death. In contrast, tumor suppressors have opposite functions like inhibiting cell division or causing cell death. Aberrations in DNA repair genes cause inappropriate DNA repair, which leads to accumulation of mutations and cancer. Although the affected genes may differ between tumors, the cancer cells share most of the characteristics, such as changes in cell morphology, insensitivity to anti-growth signals, evasion of apoptosis, continuous division and gain of migratory, invasive and metastatic properties. (Hanahan and Weinberg 2011, Sandoval and Esteller 2012). 
Traditionally, cancer was considered as a genetic disease, but it is now realized that a combination of epigenetic abnormalities and genetic alterations work together to promote cancer development and progression (Jones and Laird 1999, Feinberg, Ohlsson et al. 2006, Jones and Baylin 2007).

\subsection{Chromatin Structure and organization}

Eukaryotic cells contain approximately two-meters-long genomic DNA, which is compacted and packed in the form of chromatin into $10 \mu \mathrm{m}$ nucleus. However, it can be accessible to the cellular processes such as gene expression and regulation at the same time (Felsenfeld and Groudine 2003).

Chromatin is a complex of DNA, histones and other proteins. It is a highly ordered structure made up of arrays of nucleosomes that forms the genetic material of a cell (Burgoyne, Mobbs et al. 1976). The nucleosome is the repeating unit of chromatin that comprises of approximately 147 base pairs of DNA wrapped in about 1.67 turns around the octamer of core histones. The core histones that form the octamer are $\mathrm{H} 2 \mathrm{~A}, \mathrm{H} 2 \mathrm{~B}, \mathrm{H} 3$ and $\mathrm{H} 4$ and each present as two copies within the nucleosome (Kornberg 1974, Fletcher and Hansen 1996, Luger, Mader et al. 1997, Suganuma and Workman 2011). Core histones contain positively charged lysine and arginine residues and their basic nature that allows them to be wrapped by the negatively charged DNA, which help in maintaining the stability of nucleosome. Within the histone octamer, histone proteins are known to dimerize through a structural feature called, histone fold, which constitutes three alpha-helices. In addition to the histone fold, histone possesses $\mathrm{N}$-terminal tails that project out of the nucleosome and are known to contribute to the overall structural organization of the chromatin due to their 
propensity to be modified post-translationally (Fletcher and Hansen 1996, Luger, Mader et al. 1997).

Each nucleosome is joined to the other through a linker DNA (180-240 bp) which is in turn bound by a fifth type of histone called linker histone H1. The linker histones are highly basic and bind to the DNA in the nucleosome core particles through their globular domains and the linker DNA with their tails (Das, Lucia et al. 2009, Vempati, Jayani et al. 2010, Suganuma and Workman 2011). The linker histones play a major role in the secondary level of chromatin organization by connecting adjacent nucleosomes to form a fiber-like structure, chromatin (Allan, Cowling et al. 1981, Fletcher and Hansen 1996, Dutnall and Ramakrishnan 1997, Luger, Mader et al. 1997, Thomas 1999) (Fig.1).

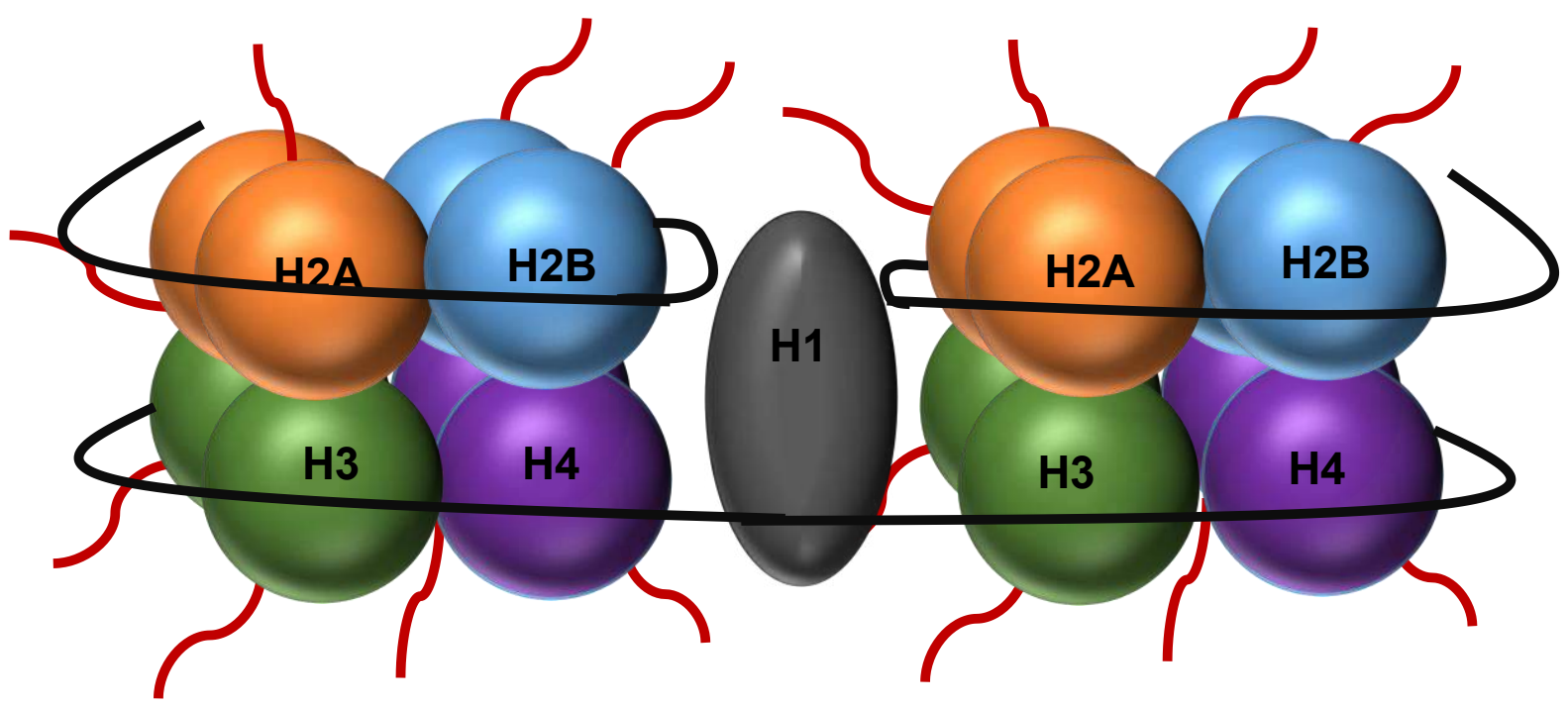

Figure. 1: The structure of the nucleosome. The basic structure of chromatin, nucleosome comprises of an octamer of histones $\mathrm{H} 2 \mathrm{~A}, \mathrm{H} 2 \mathrm{~B}, \mathrm{H} 3$ and $\mathrm{H} 4$ as dimers. Histones are wrapped around by 147 base pairs of DNA. The two nucleosomes are joined together by linker DNA and $\mathrm{H} 1$ binds to adjacent nucleosomes. The histone tails undergo posttranslational modifications.

The most striking property of chromatin is the way it is packed within a cell. Nucleosome along with linker DNA form linear $11 \mathrm{~nm}$ fiber-like structure, where 
nucleosomes are arranged as "beads on a string" which is further compacted into a thicker fiber with $30 \mathrm{~nm}$ diameter (Kornberg 1974, Marsden and Laemmli 1979, Turner 1993). The $30-\mathrm{nm}$ fiber achieves a compaction of roughly 50 -fold from the original DNA length, which is further packed to form the higher-order structures (Fig. 2) (Henikoff 2000, Richards and Elgin 2002, Felsenfeld and Groudine 2003).

Chromatin can be divided into two major states, "euchromatin" and "heterochromatin" Heterochromatin is a highly condensed form of chromatin that is inaccessible to DNA binding factors and transcriptionally inactive. In contrast, euchromatin is less condensed, open and easily accessible, comprises a high density of genes that are either actively transcribed or repressed (Owen-Hughes and Bruno 2004, Grewal and Elgin 2007). Heterochromatin is also known as a chromatin state that functions in controlling chromosomal stability and preventing mutations and translocations (Huang, Fan et al. 2004, Muegge 2005, Talbert and Henikoff 2006). 

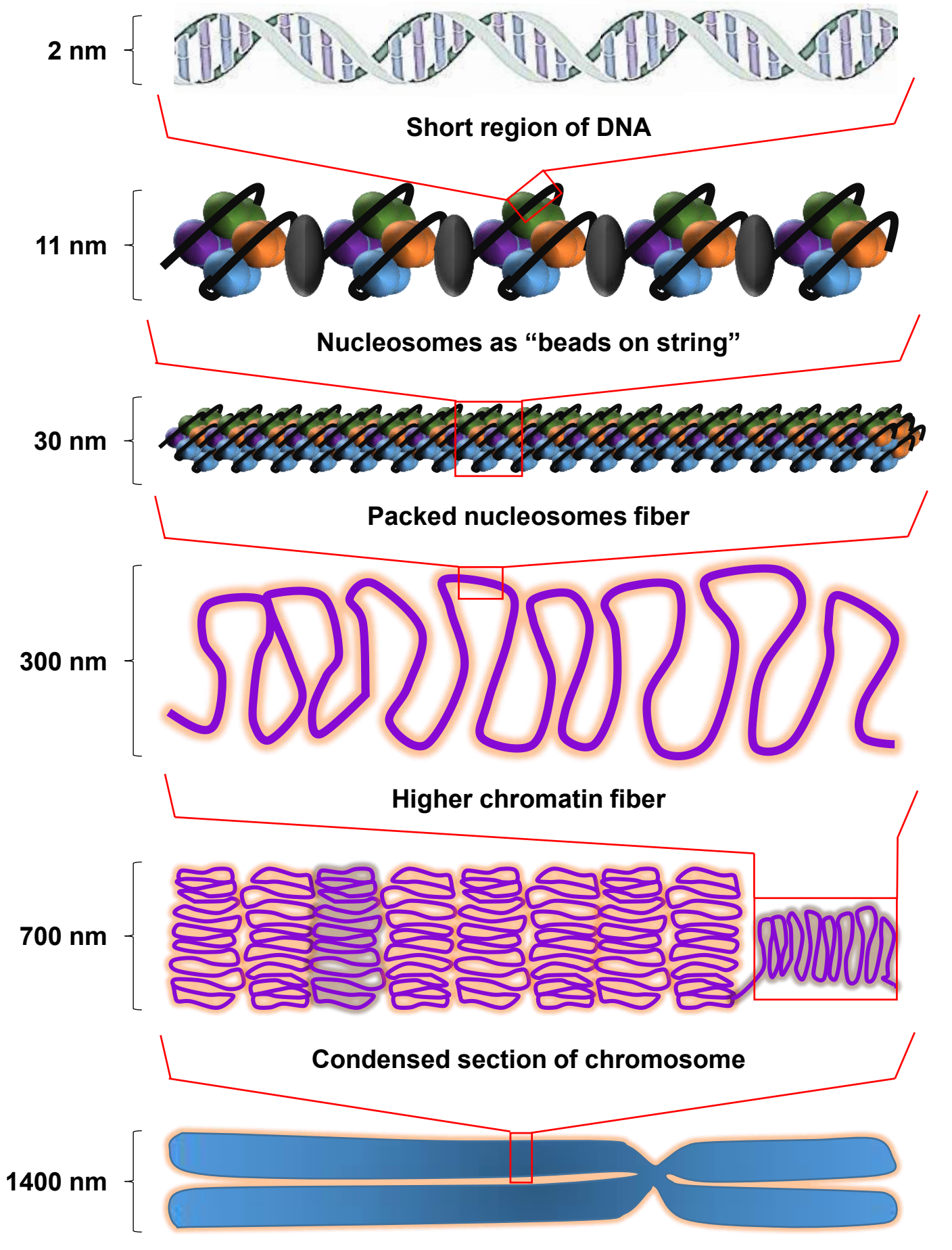

Chromosome

Figure. 2: Chromatin organization. DNA is wrapped around a histone octamer to form nucleosome and the two nucleosomes are connected by linker DNA to form $11 \mathrm{~nm}$ chromatin fiber. The chromatin fibers are condensed to form $30 \mathrm{~nm}$ fiber and then compacted to form higher order structures. Modified from (Felsenfeld and Groudine 2003) 


\subsection{Histone modifications}

In general, the core histones are subjected to different post-translational modifications in their $\mathrm{N}$ - and C-terminal tails, which are unstructured and freely accessible on the surface of the nucleosome core to various enzymatic machineries to act at specific amino acid residues (Luger, Mader et al. 1997, Jenuwein and Allis 2001, Rando and Chang 2009). The post-translational modifications include methylation of lysine and arginine residues, acetylation of lysine residues and phosphorylation of serine and threonine residues, which are well studied. There is also poorly understood category of the post-translational modifications, which includes ubiquitination and sumoylation of lysine residues (Fig.3) (Fischle, Wang et al. 2003, Osley 2004, Yang 2005, Johnsen 2012). Acetylation and methylation are the most elaborately studied modifications. Their functional consequences and the domains that recognize them are also well studied along with the enzymes that carry out these modifications. These modifications extend the histone's role in the regulation of processes like replication, transcription, recombination and repair besides scaffolding the chromatin structure (Jenuwein and Allis 2001).

The combination of post-translational modifications of histones creates a complex set of signals defined as "histone code". Before the histone code was proposed, histones were simply thought as proteins required for DNA compaction. Now it has become evident that the DNA sequence alone can not decide all the outcomes of chromatin and there are other factors that lead to various chromatin-templated events. 


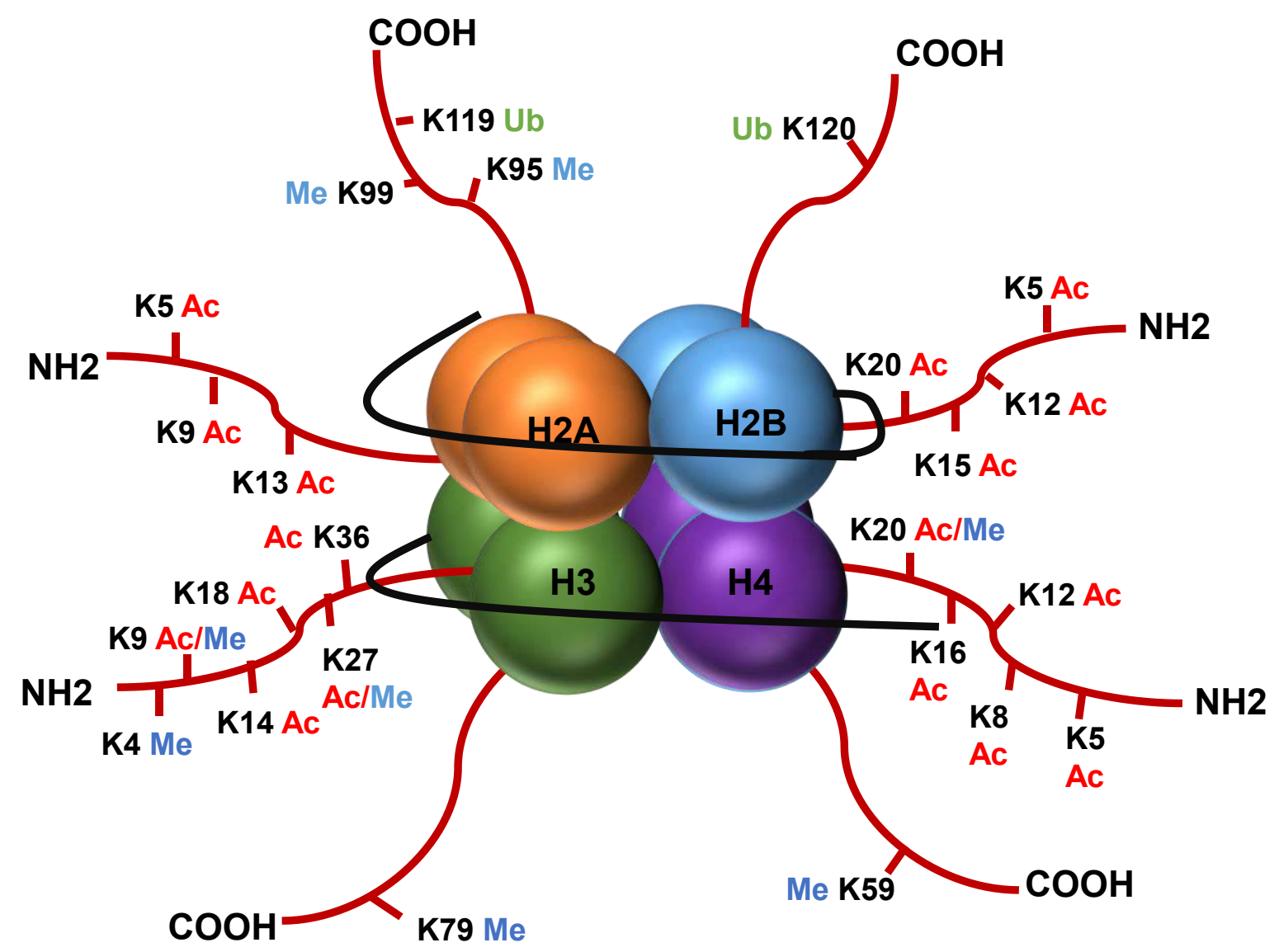

Figure. 3: Main post-translational histone modifications. Different types of posttranslational modifications occur at the $\mathrm{NH} 2$ - terminal and $\mathrm{COOH}$ - terminal tails of the histones which define the chromatin state. Histone modifications include acetylation, methylation, ubiquitination, phosphorylation and sumoylation. Some of the modifications are shown here. Modified from (Cota, Shafa et al.)

The "histone code" provides a specific binding site for selected effector proteins and can determine the cell fate by regulating key processes such as cell cycle, apoptosis and signal transduction (Strahl and Allis 2000, Jenuwein and Allis 2001, Latham and Dent 2007). Some histone modifications are associated with transcriptional silencing (H3K27me3) (Karpiuk, Najafova et al.) and others with activation (H3K4me3) (Strahl and Allis 2000, Bannister, Schneider et al. 2002). This function is mediated either directly by changing the physical or chemical properties of the chromatin fiber or indirectly by recruiting the chromatin-modifying proteins that can activate or repress the transcription. 


\subsubsection{Chromatin modifying enzymes}

Epigenetic modifications (DNA and histones) play an important role in the regulation of hetero and euchromatin. Epigenetic modifications remodel the chromatin either directly by influencing the nature of interactions with the DNA or by employing other non-histone proteins which regulate cell-specific gene expression or repression. The epigenetic regulators alter the chromatin structure and regulate its accessibility to the replication and transcriptional machinery (Luger, Mader et al. 1997, Horn and Peterson 2002, Narlikar, Fan et al. 2002). These modifications can be achieved by the specific types of enzymes and co-factors and they can be majorly classified into the following categories: "epigenetic writers" and "epigenetic erasers". Epigenetic writers are the enzymes that catalyze the addition of chemical groups onto either histone tails or the DNA such as acetyl or methyl groups or ubiquitin moieties (e.g. histone lysine acetyltransferases, histone lysine/arginine methyltransferases, and ubiquitin ligases, DNA methyltransferases) (Campos and Reinberg 2009, Cedar and Bergman 2009). Epigenetic erasers are a group of enzymes that can remove the histone modifications (e.g. histone demethylases and histone deacetylases) (Kangaspeska, Stride et al. 2008, Metivier, Gallais et al. 2008). Misregulation of factors that mediate the deposition or removal of histone modifications leads to epigenetic imbalance, which causes cancer initiation, progression and metastasis.

\subsubsection{Chromatin binding proteins}

"Epigenetic readers" are a special class of proteins that recognize and are recruited to the specific modifications on histones or nucleotides. Some of the well-studied examples are- bromodomain for acetylated lysine, chromodomain, PHD-finger 
domains and WDR40 for methylated lysine or arginine residues. (de la Cruz, Lois et al. 2005).

Chromatin is a highly dynamic structure that is constantly remodeled to provide accessibility to several factors facilitating important biological processes. In addition to the chromatin modifiers, there are chromatin remodeling enzymes and histone chaperones. that alter the chromatin architecture by removing, adding, moving or replacing histones in the chromatin (Strahl and Allis 2000, Loyola and Almouzni 2004, Taverna, Li et al. 2007, Wilson and Roberts 2011). Chromatin remodeling is known to participate in various DNA transaction processes like replication, recombination, transcription, repair and chromatin assembly. The chromatin remodeling complexes are classified into following families, such as SWI/SNF (switching defective/sucrose non-fermenting) family, the ISWI (imitation SWI) family, the NuRD (nucleosome remodeling and deacetylation)/Mi-2/CHD (chromodomain, helicase, DNA binding) family and the INO80 (inositol requiring 80) family (Morrison and Shen 2009, Hargreaves and Crabtree 2011).

\subsection{Cancer and metastatic progression}

Cancer metastasis is a complex and multistep process in which tumor cells disseminate from the primary tumor, migrate and survive during circulation, invade and adapt to the microenvironment of distant secondary site to form new tumors (secondary tumors) that result in the $90 \%$ of deaths in the cancer patients (Zajicek 1996, Gupta and Massague 2006). 


\subsubsection{Epithelial-to-mesenchymal transition}

Epithelial-to-mesenchymal transition (EMT) is first identified in the development of the embryo and it plays an essential role in the early developmental processes such as gastrulation, mesoderm formation and neural crest development. During EMT, polarized, immotile epithelial cells transform their plasticity into a migratory mesenchymal phenotype by transient dedifferentiation (Wang and Zhou 2013, Lamouille, Xu et al. 2014). To undergo EMT, the epithelial cancer cell loses the expression of E-cadherin and cell-cell adhesion proteins such as claudin, occludins and Zonula occludes 1 (ZO1) (Huang, Guilford et al. 2012). The adherent junctions are destabilized by degradation of epithelial cadherin (E-cadherin) and repression of cytokeratins. The down-regulation of E-cadherin is compensated by increased expression of mesenchymal neural cadherin ( $\mathrm{N}$-cadherin), fibronectin, Vimentin and facilitate cell migration and invasion (Maschler, Wirl et al. 2005, Kim, Litzenburger et al. 2007, Wheelock, Shintani et al. 2008, Yilmaz and Christofori 2009, Huang, Guilford et al. 2012).

EMT is also involved in wound healing, tissue regeneration and organ fibrosis (Wang and Zhou 2013). In cancer, the differentiated epithelial tumor cells convert into differentiated mesenchymal cells through the EMT process. EMT phenotype is associated with a reduction in tumor growth, increased resistance to apoptosis, increased motility and invasiveness and enhanced metastatic ability (Kang and Massague 2004, Eccles and Welch 2007).

During EMT, a set of genes, associated with cell adhesion, differentiation, migration and invasion, are transcriptionally altered which is achieved by several transcription factors, SNAL1, SLUG, TWIST1, ZEB1, ZEB2 and basic helix-loop-helix factors. 
Depletion of E-cadherin is an important characteristic of EMT during the development and cancer progression. SNAL1 repress $\mathrm{CDH} 1$ gene transcription by directly binding to the E-boxes of $\mathrm{CDH} 1$ promoter. In addition to SNAIL1, several other transcription factors are also capable of repressing E-cadherin transcription. SLUG, a close relative of SNAL1 and two other members of ZEB family- ZEB1 and ZEB2 also bind to the $\mathrm{CDH} 1$ promoter and downregulate the E-cadherin expression (Hennig, Behrens et al. 1995, Giroldi, Bringuier et al. 1997, Batlle, Sancho et al. 2000, Cano, Perez-Moreno et al. 2000, Comijn, Berx et al. 2001, Hajra, Chen et al. 2002, Eger, Aigner et al. 2005). These transcription factors act as transcriptional repressors and suppress the expression of E-cadherin and ZO-1, leading to the dissolution of cell-cell adhesions and tight junctions. Repression of epithelial markers is balanced by upregulation of mesenchymal markers, such as $\mathrm{N}$-cadherin, Vimentin and fibronectin. $\mathrm{N}$-cadherin upregulation stimulates the tumor metastasis and its expression is associated with poor survival. Vimentin is an intermediate filament which is involved in the migration and invasiveness and its expression is considered as a charcteristic of epithelial cells undergoing EMT (Franke, Grund et al. 1982, Nakajima, Doi et al. 2004). Altogether, these changes enable the cells to switch from an epithelial phenotype to mesenchymal phenotype by regulating expression of epithelial markers to promote cell migration and invasion.

\subsubsection{Mesenchymal-to-Epithelial transition}

Mesenchymal-to-Epithelial transition (MET) is a reverse-EMT process in which motile, multipolar or spindle- shaped mesenchymal cells are transformed into polarized epithelial cells. In general, this process occurs after the cell reaches the destination followed by their differentiation into specific tissue resident cells during 
embryo development or organogenesis (De Craene and Berx 2013, Nieto 2013). But in the case of cancer, the migrated mesenchymal tumor cells revert back into epithelial cells through MET process and form a secondary tumor (Peinado, Olmeda et al. 2007, Yang, Chen et al. 2009, Martin, Dwyer et al. 2010, Sun, Zhao et al. 2010).

EMT and MET consist the opposite processes by which cells switch between epithelial and mesenchymal phenotypes and play a vital role both in embryo development and organogenesis and in tumor metastasis. As the critical EMT event is the down-regulation or repression of E-cadherin, the reexpression of E-cadherin is an important hallmark of MET. (Fig.4). Both EMT and MET are dynamically balanced to maintain tissue homeostasis and development of embryo and metastatic cancer (Prindull 2005, Ricci-Vitiani, Lombardi et al. 2007, Thiery, Acloque et al. 2009)

MET participate in the stability of distant metastases by making the cancerous cells regain epithelial properties and integrate into distant organs (Micalizzi, Farabaugh et al. 2010, Heerboth, Housman et al. 2015). MET is also an essential developmental process and well-studied in kidney organogenesis, cardiogenesis, hepatogenesis and somitogenesis (Nakajima, Yamagishi et al. 2000, Nakaya, Kuroda et al. 2004, Li, Zheng et al. 2011).

\subsubsection{Cancer stem cells and metastasis}

Cancer stem cells (CSCs) or tumor-initiating cells represent a small percentage of tumor cells that have an ability to self-renew and differentiate into cancer cells (Lapidot, Sirard et al. 1994, Jaggupilli and Elkord 2012). 

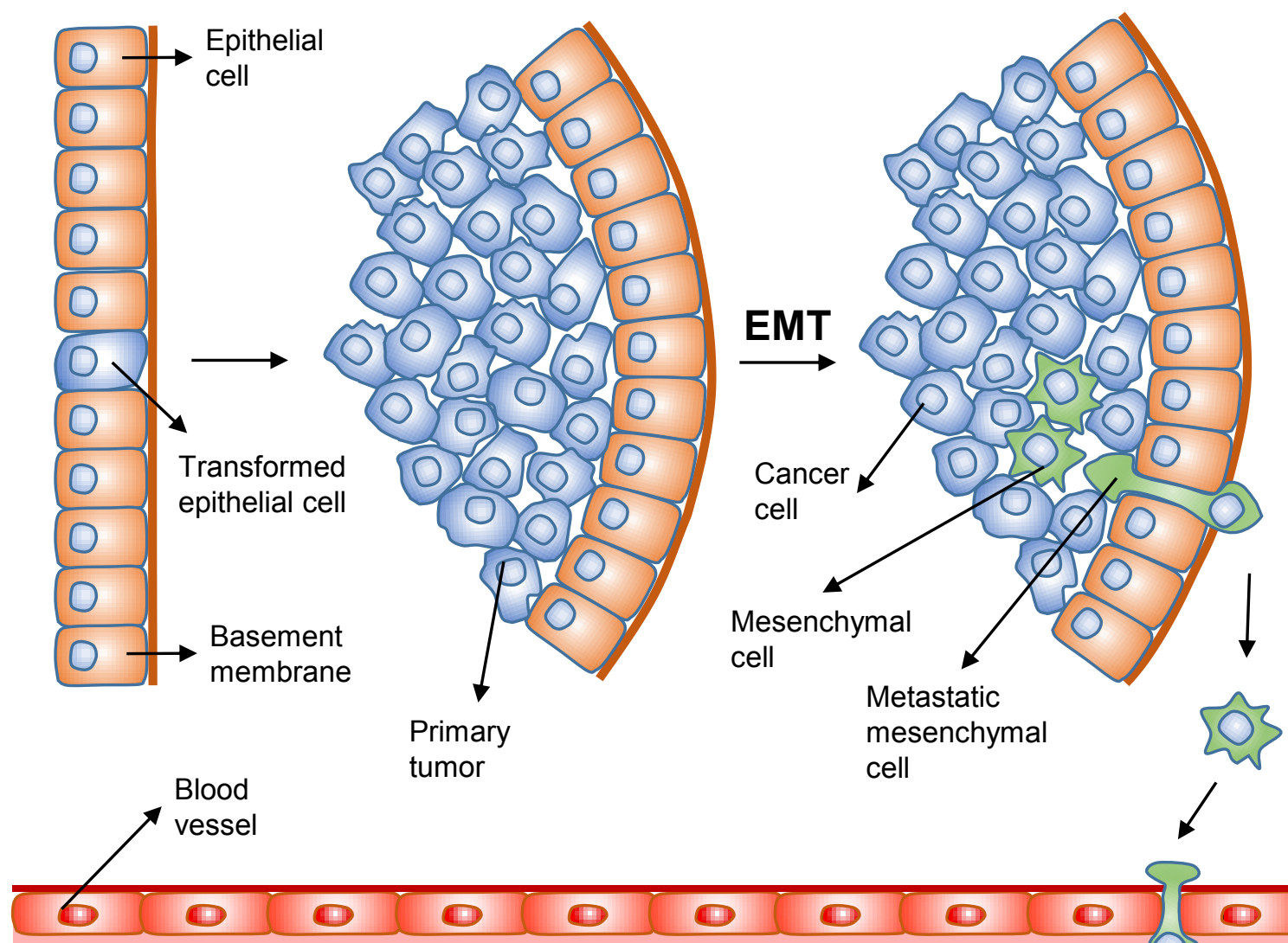

tumor cell

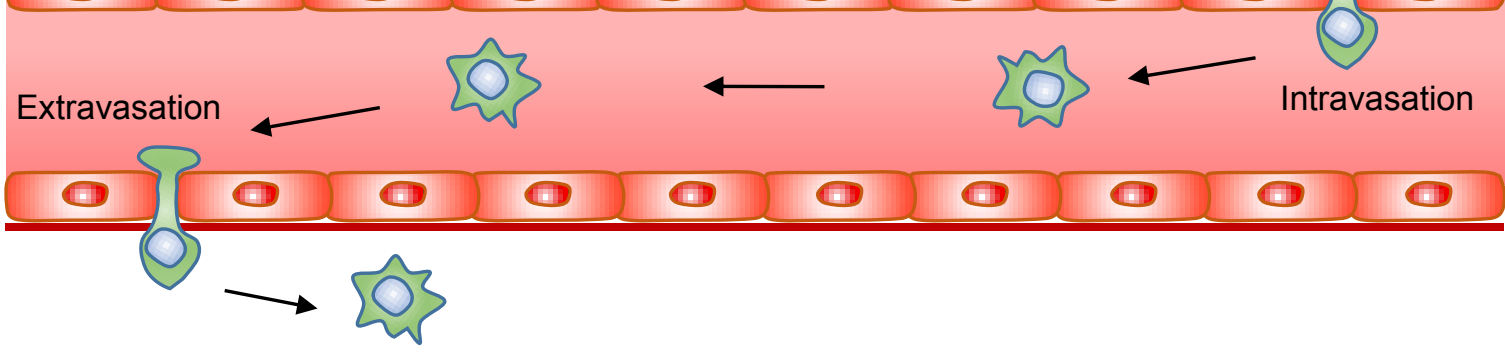

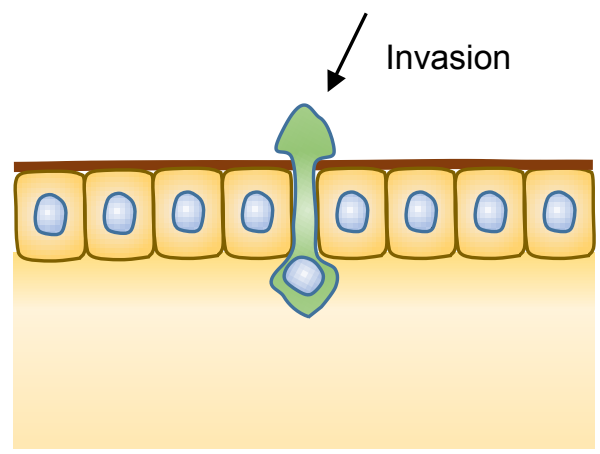

Distant organ

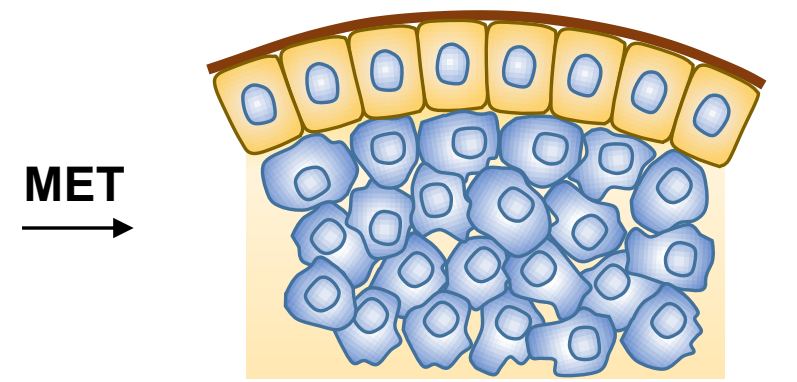

Secondary tumor

Figure. 4: EMT and MET in carcinogenesis and tumor metastasis. The transformed epithelial cell divide, grow and form a primary tumor. The epithelial cells are converted to mesenchymal cells through EMT process. Then the tumor cells disseminate from the primary tumor and enter into circulation (intravasation). The disseminated tumor cells exist the blood vessels (extravasation) and invade into the distant organ and the mesenchymal cells revert back into epithelial cells through MET to form a secodary tumor. Modified from (Peinado, Olmeda et al. 2007). 
Similar to the normal stem cells, CSCs can also develop into different types of cancer cells in the tumor and hence termed as cancer stem cells. CSCs are capable of dictating invasion, oncogenesis, metastasis, tumor growth and cancer recurrence. Differentiated cancer cells were shown to proliferate faster than CSCs, but have the little tumor-initiating capacity (Chen, Kasai et al. 2012). CSCs can form new tumors when injected into immunodeficient mice (Chen, Yang et al. 2012). CSCs are first identified in leukaemia and have been reported in different cancers including breast (Al-Hajj, Wicha et al. 2003), colon (Ricci-Vitiani, Lombardi et al. 2007), prostrate (Gu, Yuan et al. 2007), ovarian (Zhang, Balch et al. 2008), pancreas and other tissues (Lapidot, Sirard et al. 1994, Visvader and Lindeman 2008).

CSCs can be identified by both CSC-specific cell surface marker expression and also by functionality assays, such as sphere formation and aldehyde dehydrogenase (ALDH) activity assays. The surface marker expression profile of CSCs, that promotes breast cancer metastasis, is CD44 $4^{\text {high }} / \mathrm{CD} 24^{\text {low }}$ (Al-Hajj, Wicha et al. 2003). Signal transduction pathways of stem cell regulation also play an important role in carcinogenesis e.g. Notch, Sonic hedgehog (Gigi, Geiger et al.), and Wnt signaling. The traditional therapies used against cancer such as chemotherapy and radiotherapy are inefficient due to drug resistance of cancer cells and self-renewal properties of CSCs, which leads to cancer recurrence (Kopper and Hajdu 2004, Dragu, Necula et al. 2015).

\subsection{Epigenetics in cancer}

Cancer is initiated by genetic alterations such as mutations or by epigenetics, such as tumor suppressors and oncogenes, which regulate cell survival, proliferation and homeostatic functions. Epigenetic mechanisms play an indispensable role in normal 
development and maintenance of tissue-specific gene expression. (Egger, Liang et al. 2004, Sharma, Kelly et al. 2010). Disruption of epigenetic processes alters gene expression and malignant cellular transformation (Sharma, Kelly et al. 2010). The post-translational modifications of histones play a crucial role in regulating the accessibility of epigenetic regulators and transcription factors to the specific regions of chromatin. Tumorigenesis is hypothesized that alteration in the epigenetic modifications leads to inappropriate expression or silencing of genes (oncogenes or tumor suppressors) (Chi, Allis et al. 2010).

Following is a brief overview of different epigenetic modifications, the enzymes involved in maintaining their dynamics and their functional relevance.

\subsubsection{DNA methylation}

DNA methylation is one of the fundamental and most intensely studied epigenetic modifications, which plays an important role in regulating gene expression and stable gene silencing. DNA methylation is associated with histone modifications and regulate the genomic function by changing chromatin architecture. DNA methylation is a covalent modification of DNA that occurs at the cytosine residues in CpG dinucleotides, which form large clusters called CpG islands. DNA methylation is catalyzed by a family of DNA methyltransferases (DNMTs). CpG islands occupy approximately $60 \%$ of human gene promoters (Bird 2002, Takai and Jones 2002, Wang and Leung 2004).

Cancer cells exhibit aberrant DNA methylation patterns such as hypo and hypermethylation, which can drive malignant transformation. Tumor suppressor genes are often silenced in tumor cells due to hypermethylation whereas oncogenes are hypo methylated (Gonzalo 2010, Zhang, Cardarelli et al. 2011). DNMTs are 
overexpressed in breast cancers. It was reported that DNA methylation silences several genes in breast cancer and thus affects several pathways involved in breast tissue homeostasis, including cell cycle regulation, tumor susceptibility, carcinogen detoxification and cell adhesion (Widschwendter and Jones 2002).

E-cadherin $(\mathrm{CDH} 1)$ was reported to be downregulated by DNA methylation promoting an invasive phenotype in prostate and other epithelial cancers. It was shown that hypermethylation of $\mathrm{CpG}$ island in the promoter region of the $\mathrm{CDH} 1$ gene alters the expression of E-cadherin, leading to loss of tissue integrity which is an important step in tumor progression. (Caldeira, Prando et al. 2006, Keil, Abler et al. 2014). DNA methylation and histone modifications cooperate with each other to repress the target gene expression. E-Cadherin expression is significantly downregulated by suppressing demethylation of histone $\mathrm{H} 3$ on lysine 9 (H3K9me2) and DNA methylation by an interaction of EMT-TF, SNAIL with G9a and DNA methyltransferases (DNMTs) and recruits to the promoters (Dong, Wu et al. 2012). It was also reported that DNA methylation is involved in the generation of CSCs (Esquela-Kerscher and Slack 2006, Volinia, Calin et al. 2006). Altogether, alteration in DNA methylation plays an important role during the EMT induction.

\subsubsection{Histone modifications and their functional consequences}

Alteration in the post-translational modifications of histones contributes to the repression of tumor suppressor genes and the activation of oncogenic genes. Several histone readers and writers are mutated or transcriptionally altered in tumors. Histone modifications like acetylation, methylation and ubiquitination were shown to be associated with several disorders and cancers (Fraga and Esteller 2005, Seligson, Horvath et al. 2009). 


\subsubsection{Histone acetylation}

Histone acetylation is a reversible, very dynamic and well-characterized modification which is involved in chromatin structure, transcription, DNA repair and cancer progression (Wang, Allis et al. 2007, Choudhary, Kumar et al. 2009). Histone acetylation is a chemical reaction carried by various histone acetyltransferases (HATs), which transfer the acetyl group from acetyl coenzyme A (acetyl-CoA) to the $\varepsilon$-amino group of the lysine residues of histone proteins. Histone acetylation is generally associated with a more open chromatin structure and active transcription. The acetylation of histones neutralizes the positive charge of lysines, which can potentially reduce the interaction between DNA and histones and thereby increases DNA accessibility to the transcription factors (Imhof, Yang et al. 1997). Histone acetylation regulates many cellular processes and the unique acetylation marks either alone or in combination lead to distinct outcomes (Grunstein 1997, Tropberger and Schneider 2010).

The most prominent members of the acetyl transferases are the GNAT (Gcn5$\begin{array}{llll}\text { related } \quad \mathrm{N} \text {-acetyltransferases), } & \mathrm{CBP} / \mathrm{p} 300 \quad \text { and } & \text { MYST }\end{array}$ (MOZ/YBF2/SAS2/TIP60/HBO1) families. Broadly each of these enzymes is capable of modifying different lysine residues of histones. HATs can act as tumor suppressors and control cell cycle progression and proliferation. They can also function as oncogenes and activate malignant genes by altering acetylation profile and contribute to cancer (Di Cerbo and Schneider 2013). The HAT, p300 promotes EMT and tumor progression by affecting the regulation of SNAIL and ZEB1 in colon cancer (Pena, Garcia et al. 2006). The HATs such as KAT2A, KAT2B and KAT5 
acetylate the oncogene c-MYC and leads to cancer progression (Patel, Du et al. 2004).

\subsubsection{Histone deacetylation}

Deacetylation is a reverse reaction to acetylation, catalyzed by Histone deacetylases (HDACs), which restores the positive charge of the lysine residues. Histone deacetylation potentially results in chromatin compaction and transcriptional repression (Yang and Seto 2007). Eighteen mammalian HDACs are reported till now and are categorized into four major classes based on their sequence homology and cofactor dependency. Class I includes HDACs 1, 2, 3 and 8 (similar to yeast Rpd3), Class II consists HDAC 4, 6, 7, 9 and 10 (similar to yeast Hda1), Class III includes sirtuins, SIRT1-7(similar to yeast Sir2) and class IV consists of HDAC 11 (Ropero and Esteller 2007).

Loss of histone acetylation is not only involved in carcinogenesis but also in tumor invasion and metastasis (Yasui, Oue et al. 2003). Like HATs, HDACs are also important for breast cancer development. HDAC1 plays an important role in TGF $\beta 1$ induced EMT and inhibition of HDAC1 suppress TGF 11 induced EMT (Yoshikawa, Hishikawa et al. 2007, Lei, Zhang et al. 2010). HDAC3 interacts with a component of histone methyltransferase complex, WDR5 and induces hypoxia-mediated EMT by regulating acetylation and methylation patterns on EMT genes. SIRT1 was shown to interact with ZEB1 and repress CDH1 expression by deacetylation of its promoter (Wu, Tsai et al. 2011, Byles, Zhu et al. 2012). HDAC1/2 complex cooperates with either SNAIL or ZEB1 and recruited to the $\mathrm{CDH} 1$ promoter and represses its expression. 


\subsubsection{Histone methylation}

Histone methyltransferases (HMTs) transfer methyl groups from S-adenosyl methionine to the side chain of lysine and arginine residues. As methyl groups do not carry any charge on them, histone methylation does not alter the overall charge of the histone proteins. Lysines residues may be mono-, di-, or tri-methylated whereas arginines may be mono-, symmetrically or asymmetrically dimethylated (Bedford and Clarke 2009). The methyltransferases are classified into arginine (PRMTs) or lysine (KMTs) methyltransferases depending on the methylation of the amino acid residue. SET domain is the catalytic subunit of all histone methyltransferases (HMTs) such as G9a, EZH1/2, SUV39H1/H2, except for the DOT1L, H3K79 methyltransferase, and transfer methyl group (Miller, Krogan et al. 2001, Wang and Shang 2013). Lysines $4,9,27,36$ and 79 of histone $\mathrm{H} 3$ and 20 of histone $\mathrm{H} 4$ are methylated by lysine methyltransferases. Histone methylation is known to be associated with either transcriptional activation or repression depending upon the position of the amino acid residue modified. For example, trimethylation of histone H3 at K4 (H3K4me3), K79 (H3K79me3) and $\mathrm{K} 36$ (H3K36me3) are considered as active marks, whereas $\mathrm{H} 3 \mathrm{~K} 9 \mathrm{me} 2, \mathrm{H} 3 \mathrm{~K} 9 \mathrm{me} 3$ and $\mathrm{H} 3 \mathrm{~K} 27 \mathrm{me} 3$ are considered as transcriptional repressive marks (Kouzarides 2007).

Histone methylation is important for proper genome programming during development. Misregulation of methylation machinery can lead to diseased states such as cancer. Several studies reported that altered histone methylation might play a role in cancer, tumor recurrence and poor survival (Albert and Helin 2010, Chi, Allis et al. 2010). Aberrant regulation of G9a (H3K9 methyltransferase) was identified in several cancers. EMT-TF, SNAIL cooperates with histone methyltransferase G9a 
and DNMTs to the $\mathrm{CDH} 1$ gene promoter to modulate CDH1 expression (Dong, Wu et al. 2012). It is also reported that Suv39H1 can trimethylate H3K9 on the CDH1 promoter (Serrano-Gomez, Maziveyi et al. 2016). Other methyltransferases, MMSET can di- or trimethylate H3K36 at the TWIST promoter whereas SET8 methylates $\mathrm{H} 420$ at the $\mathrm{CDH} 1$ and $\mathrm{CDH} 2$ promoters (Serrano-Gomez, Maziveyi et al. 2016). Mesenchymal markers are marked with H3K4me3 by WDR5, part of MLL and SET1 HMT complex upon hypoxia (Wu, Tsai et al. 2011). The PRC2 complex which contains methyltransferase along with EED and SUZ12 plays a crucial role in transcriptional silencing by a repressive mark, $\mathrm{H} 3 \mathrm{~K} 27 \mathrm{me} 3$ in carcinogenesis and EMT (Orlando 2003, Herranz, Pasini et al. 2008)

\subsubsection{Histone demethylation}

Since methylation is a very specific and stable mark, it was initially thought to be an irreversible modification until the discovery of the lysine demethylase, LSD1 or KDM1A which can remove mono and dimethyl groups from $\mathrm{K} 4$ of histone $\mathrm{H} 3$ (H3K4me1/2), leading to transcription repression (Shi, Lan et al. 2004). JMJD6, a jumonii domain containing protein is the first arginine demethylase, which can demethylate methyl group from R2 and R3 of histone H3 (Chang, Chen et al. 2007). LSD1 is recruited to the epithelial gene promoters and repress the gene expression by removing the methyl groups from the H3K4me2 by interacting with EMT-TF, SNAIL (Lin, Ponn et al. 2010, Amente, Lania et al. 2013, Ferrari-Amorotti, Fragliasso et al. 2013). A histone H3K4 demethylase, KDM5B increases the expression of transcription factors ZEB1 which further downregulates the E-cadherin expression and upregulates the mesenchymal marker genes (Enkhbaatar, Terashima et al. 2013). Two other demethylases, JMJD3 (KDM6B) and JMJD2B (KDM4B) can 
demethylate $\mathrm{H} 3 \mathrm{~K} 27 \mathrm{me} 3$ and $\mathrm{H} 3 \mathrm{~K} 9 \mathrm{me} 3$ respectively and were shown recently to promote EMT (Ramadoss, Chen et al. 2012, Zhao, Li et al. 2013).

\subsubsection{Histone ubiquitination}

Ubiquitination is a basic biochemical process of covalent attachment of one or more ubiquitin molecules to lysine residues of proteins or to existing ubiquitin molecules on a protein. Ubiquitination is catalyzed by the sequential action of three enzymes, E1 ubiquitin-activating, E2 ubiquitin-conjugating and E3 ubiquitin-ligase enzymes (Hershko and Ciechanover 1998, Sadowski and Sarcevic 2010). Ubiquitination controls the protein functions, such as degradation and subcellular localization (Pickart and Eddins 2004). Monoubiquitination can regulate DNA repair and gene expression but polyubiquitination through UbK48 targets proteins for proteasome degradation (Passmore and Barford 2004).

$\mathrm{H} 2 \mathrm{~A}$ monoubiquitination is carried by PRC1 complex, which comprises RING1A and RING1B (Wang, Wang et al. 2004, Cao, Tsukada et al. 2005). H2B monoubiquitination is performed by RNF20/40 heterodimer (Zhu, Zheng et al. 2005). Ubiquitination plays a vital role in PRC-mediated silencing (Niessen, Demmers et al. 2009). Both histones, $\mathrm{H} 2 \mathrm{~A}$ and $\mathrm{H} 2 \mathrm{~B}$ are monoubiquitinated at $\mathrm{K} 119$ (H2Aub1) and K120 (H2Bub1) respectively. H2Aub1 is associated with heterochromatin and prevents transcription elongation, whereas H2Bub1 is localized to euchromatin and promotes transcription elongation (Wang, Wang et al. 2004, Cao, Tsukada et al. 2005, Xiao, Kao et al. 2005, Shukla, Stanojevic et al. 2006, Minsky, Shema et al. 2008). H2Bub1 physically disrupt chromatin structure and makes DNA accessible to transcription factors and DNA repair proteins and plays a vital role in transcription, DNA damage response and also stem cell differentiation. 
Decreased H2Bub1 levels were reported to be associated with tumor progression and tumor invasion (Shema, Tirosh et al. 2008, Prenzel, Begus-Nahrmann et al. 2011, Johnsen 2012). Global H2Bub1 levels are either decreased or absent in advanced cancers such as breast, lung, colorectal and parathyroid cancers. The components of the PRC1 complex ubiquitinates $\mathrm{H} 2 \mathrm{~A}$ and promotes EMT by upregulating SNAIL through regulating PI3K/Akt/GSK-3 $\beta$ signaling pathway and also targets other EMT-TFs, Twist1 and ZEB1 (Song, Li et al. 2009, Wellner, Schubert et al. 2009, Yang, Hsu et al. 2010).

\subsubsection{Deubiquitination}

As other histone modifications, ubiquitination is also a reversible process and the removal of ubiquitin molecules is termed as deubiquitination which is performed by deubiquitinases (DUBs). There are around 100 DUBs encoded by the human genome, which are classified into 5 families. They are ubiquitin C-terminal hydrolases (Firestein, Bass et al.), ubiquitin-specific proteases (USPs), ovarian tumor domain DUBs, machado Joseph domain DUBs and JAB1/MPN metalloenzyme. The activity of these enzymes affects the half-life, activity and localization of multiple proteins, which in turn regulate cell homeostasis, protein stability and signaling pathways. DUBs can also be categorized into oncogenes or tumor suppressors as they regulate the activity of the other proteins involved in tumor development and progression (Fraile, Quesada et al. 2012). EMT-TFs such as Snail/Slug, ZEB1/ZEB2, and Twist1 protein levels are tightly controlled by the ubiquitin-proteasome system (UPS) and several E3 ubiquitin ligases are shown to play crucial roles in the regulation of EMT. Genetic aberrations and alterations of these ligases have been detected in human cancer (Liu, Yang et al. 2011). USP22 
(Ubiquitin-specific-protease-22), an $\mathrm{H} 2 \mathrm{~B}$ deubiquitinating enzyme is upregulated in tumors with a stem cell-like phenotype exhibiting a poor patient outcome (Glinsky, Berezovska et al. 2005, Zhang, Varthi et al. 2008, Inoue, Itoh et al. 2016). BAP1 (BRCA1-Associated Protein 1), an $\mathrm{H} 2 \mathrm{~A}$ deubiquitinating enzyme, is reported as a tumor suppressor and its expression showed a negative correlation with tumorigenesis in lung cancer (Shen, Wang et al. 2016). Another report showed that H2A DUB, USP3 depletion induces mesenchymal cellular phenotype in A549 epithelial lung cells (Nicassio, Corrado et al. 2007, Buus, Faronato et al. 2009).

\subsection{Role of signaling pathways in EMT}

Several molecular signaling pathways play an important role in cancer development, progression and maintenance of CSC phenotype. Cell signaling pathways such as Wnt- $\beta$-catenin, Notch, transforming growth factor $\beta$ (TGF $\beta$ ), Sonic Hedgehog (Gigi, Geiger et al.), PI3K/Akt and Hypoxia are involved in the progression of EMT. These pathways are activated by various dynamic stimuli from the microenvironment, including cytokines, hypoxia etc.

\subsubsection{TGF- $\beta$ signaling pathway}

Transforming growth factor- $\beta$ (TGF- $\beta$ ) signaling is a well-studied pathway and plays a crucial role in diverse cellular processes like proliferation, apoptosis, differentiation and development. Misregulation of TGF- $\beta$ leads to defects in developmental processes, organ fibrosis and cancer. TGF- $\beta$ acts as a tumor suppressor in normal cells. Interestingly, it functions as a tumor promoter in advanced cancers. (Derynck and Akhurst 2007, Guo and Wang 2009, Nagaraj and Datta 2010). The signaling is initiated by TGF- $\beta$ binding to the transmembrane receptors that have serine/threonine kinase activity. After TGF- $\beta$ binding to the TGF- $\beta$ receptor II and 
TGF- $\beta$ receptor I is recruited into the complex and phosphorylated. This leads to the phosphorylation of the C-terminus of the receptor-regulated Smad proteins (RSmads). The activated R-Smads interact with other Smads (Co-Smads), Smad 4 and the Smad complex is translocated into the nucleus where it directly binds to the DNA and regulates TGF- $\beta$ target gene expression along with other DNA binding factors, like ZEB, SNAIL and Twist (Massague 2000, Shi and Massague 2003, Zavadil and Bottinger 2005, Gomis, Alarcon et al. 2006, Fuxe, Vincent et al. 2010). This results in the decreased expression of epithelial markers, E-cadherin and cytokeratins and upregulation of mesenchymal markers, Vimentin, N-cadherin and fibronectin.

TGF- $\beta$ is a potent driver of cancer progression through EMT induction which in turn is associated with metastatic cancer (Wood, Parsons et al. 2007, Jones, Zhang et al. 2008, Lamouille, Xu et al. 2014). TGF- $\beta$ stimulation in normal and transformed mammary epithelial cells generates cells with the capacity to propagate new tumor (Mani, Guo et al. 2008). TGF- $\beta$ induced EMT can also regulate cancer cells to dedifferentiate and gain CSC properties (Caja, Bertran et al. 2011, Fernando, Malfettone et al. 2015). Mutations in the genes encoding TGF- $\beta$ receptors and Smads can also result in carcinogenesis (Samanta and Datta 2012, Katsuno, Lamouille et al. 2013).

\subsubsection{Notch signaling pathway}

Notch signaling pathway is important for maintaining a balance between cell proliferation and apoptosis. Notch signaling regulates several cellular processes like cell proliferation, differentiation, development and cell death. It also affects the development and function of several organs. It also plays a role in neuronal function, 
embryonic development, angiogenesis, cardiac homeostasis and bone regeneration. It is also essential for the development of normal mammary gland (Miele and Osborne 1999, Miele 2006, Miele, Miao et al. 2006, Pannuti, Foreman et al. 2010, Ranganathan, Weaver et al. 2011). Activation of Notch signaling starts with the interaction between ligands such as Delta-like (Delta-like-1, -3 and -4) and Jagged (Jagged1 and Jagged2) and Notch receptors (Notch1-4) (Nichols, Miyamoto et al. 2007, Van de Walle, De Smet et al. 2011). After binding to the ligand, the Notch receptor's intracellular domain (NCID) is cleaved and released, which is then translocated to the nucleus and regulates the transcription complexes (CBF1/CSL/RBPjk/Su(H)) (Komatsu, Chao et al. 2008).

Alteration in the Notch signaling pathway is associated with a range of multiple human disorders from developmental syndromes to adult onset diseases and cancer. Overexpression of Notch1 and their ligand jagged1 was observed in breast cancer, bladder cancer leukemia and prostate cancer (Miele and Osborne 1999, Miele 2006, Wang, Banerjee et al. 2006, Wang, Zhang et al. 2006). Overexpression of Notch-1 induces Snail and promote EMT. Notch stimulates the Slug promoter, which results in the upregulation of Slug and EMT initiation (Timmerman, GregoBessa et al. 2004, Niessen, Fu et al. 2008). Dysregulation of this pathway causes several malignancies such as T-cell leukemia and breast cancer (Gallahan and Callahan 1997, Grabher, von Boehmer et al. 2006)

\subsubsection{PI3K/Akt signaling pathway}

Phosphatidylinositol 3-kinase (PI3K)/Akt pathway plays a vital role in regulating several biological processes such as cell proliferation, cell growth, metabolism, apoptosis and also maintaining the characteristics of the tumor cell. (Kanamori, 
Kigawa et al. 2001). PI3K/Akt pathway is a multistep process, which is modulated by various proteins, including $\mathrm{PI} 3 \mathrm{~K}$, phosphoinositide-dependent kinase 1 (PDK), phosphatase and tensin homolog (PTEN) and heat-shock protein 90 (HSP90). PI3K phosphorylates phosphatidylinositol 4,5-biphosphate $\left(\mathrm{PI}(4,5) \mathrm{P}_{2}\right)$ to phosphatidylinositol 3,4,5-triphosphate $\left(\mathrm{PIP}_{3}\right)$ which recruits Akt to the membrane. At the membrane, Akt is activated by PDK1 which leads to the translocation of Akt to the nucleus. Akt mediates several cellular processes such as apoptosis, cell proliferation, transcription, and cell migration and it also plays a vital role in promoting cell survival.

Dysregulation of PI3K/Akt pathway is implicated in several diseases such as diabetes, cancer, cardiovascular and neurological disorders. Genes in the PI3K/Akt pathway are frequently altered in several human cancers. Akt1 is frequently activated in breast, prostate and ovary cancers. Akt2 is overexpressed in ovarian, pancreatic, breast and thyroid cancers (Cheng, Godwin et al. 1992, Bellacosa, de Feo et al. 1995, Ringel, Hayre et al. 2001, Testa and Bellacosa 2001). PTEN is frequently mutated or depleted in various cancers (Grille, Bellacosa et al. 2003, Mayer and Arteaga 2016). Akt has been shown to repress the expression of Ecadherin and upregulate the expression of EMT inducers, Snail, Slug, BMI-1 and EZH2 which in turn promote EMT and CSC phenotype (Huang, Zhang et al. 2011, Dong, Konno et al. 2014). As a result of these somatic alterations, PI3K/Akt pathway is aberrantly activated and is associated with cellular transformation, tumorigenesis, cancer progression, and drug resistance. 


\subsubsection{Wnt signaling pathway}

Wnt is an extremely conserved developmental signaling pathway. Wnt proteins are secreted ligands that act as local mediators to regulate many aspects of development in all animals. The Wnt signaling pathway regulates cell proliferation, morphology, cell-contacts, migration and structural remodeling (Cadigan and Nusse 1997, Reya and Clevers 2005). Wnt signaling is important for activating multiple intracellular pathways related to cell proliferation, differentiation and polarity. This pathway propagates through either canonical pathway or non-canonical pathway (Bhanot, Brink et al. 1996, Yang-Snyder, Miller et al. 1996, He, Saint-Jeannet et al. 1997). The wnt proteins interact with the transmembrane receptor, Frizzled (FZD) (along with co-receptor LRP), which activates Disheveled (Dvl) results in the formation of the activated receptor complex. The complex then triggers removal of

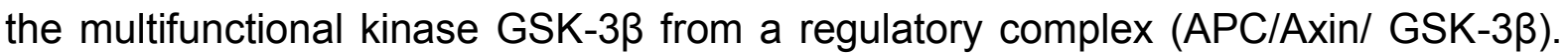
Stabilized $\beta$-catenin then translocates to the nucleus, where it binds to LEF/TCF transcription factors to activate transcription of the target genes (Pandur, Maurus et al. 2002, Logan and Nusse 2004).

Wnt pathway plays a vital role in both developmental and cancer-related EMTs (Heldin, Vanlandewijck et al. 2012). Wht signaling also plays a basic role in reprogramming and maintenance of CSC phenotype that is activated by EMT. Mutations in Wnt genes or components of Wnt pathway results in cancer progression, including tumor initiation, tumor growth, cell senescence, cell death, differentiation and metastasis. Aberrant Wnt signaling was reported to be associated with several tumors (Anastas and Moon 2013, Kim and Kahn 2014). $\beta$-catenin, along with TCF/LEF binds to the promoter region of SNAIL1 and activates its transcription 
(Easwaran, Pishvaian et al. 1999, ten Berge, Koole et al. 2008). Abnormal activation of Wnt/ $\beta$-catenin pathway also strongly correlates with carcinogenesis and cancer progression through maintaining cancer initiating cells (CICs) (Nguyen, Vanner et al. 2012).

\subsection{Additional Sex Comb-Like (ASXL), transcription regulator}

The Additional Sex Combs-like (ASXL) genes such as ASXL1, ASXL2 and ASXL3 are the mammalian homologues of the Drosophila gene, Addition of sex combs (Asx) (Fisher, Berger et al. 2003, Katoh and Katoh 2003, Katoh and Katoh 2004). The Asx protein has dual roles in the transcriptional regulation of homeotic and non-homeotic genes as a cofactor of both the Polycomb group (PcG) repressor complex and the Trithorax-group (trxG) activator complex and is involved in the transcriptional repression and activation of target genes in a context-dependent manner (Sinclair, Milne et al. 1998, Gildea, Lopez et al. 2000, Brock and van Lohuizen 2001, Dietrich, Moore et al. 2001, Grimaud, Negre et al. 2006, Halachmi, Schulze et al. 2007, Petruk, Smith et al. 2008). ASXL1 and ASXL2 are expressed in a wide variety of mammalian tissues but ASXL3 expression is confined to the brain and the eye (Fisher, Randazzo et al. 2006, Bainbridge, Hu et al. 2013, LaFave, Beguelin et al. 2015).

\subsubsection{Structure of ASXL1 protein}

The ASXL family proteins share common domains that include, an ASXN helix-turnhelix domain in the $\mathrm{N}$-terminal region, an $\mathrm{ASXH}$ globular domain in the $\mathrm{N}$-terminal adjoining region, $\mathrm{ASXM} 1$ and $\mathrm{ASXM} 2$ domains in the central part and a PHD domain in the C-terminal region. The full length human ASXL1 codes for a nuclear protein of 1541 amino acids and weighs about $170 \mathrm{kDa}$. The ASXN domain is a DNA binding 
domain and it is structurally similar to the Forkhead-box (FOX) domain of FOXA3, FOXK1, FOXO1 and FOXO4, which regulates transcription and DNA repair during embryogenesis and carcinogenesis. (Sanchez-Pulido, Kong et al. 2012, Katoh 2013). The ASXH domain directly binds to the H2A deubiquitinating enzyme BAP1, an ubiquitin carboxy-terminal hydrolase $(\mathrm{UCH})$ (Scheuermann, de Ayala Alonso et al. 2010) and Lysine Specific Demethylase-1A (LSD1/KDM1A) (Lee, Cho et al. 2010, Sanchez-Pulido, Kong et al. 2012). The ASXM1 and ASXM2 domains are proteinprotein interacting domains, which directly binds to the nuclear hormone receptors (NHRs) such as RAR, RXR, ER, AR, GR and TR as well as to the NHR-coactivator NCOA1. ASXM2 domain consists of the LVxxLL motif. (Cho, Kim et al. 2006, Grasso, Wu et al. 2012). And the PHD domain is a histone- or DNA-binding domain of chromatin regulators and transcription factors (Sanchez and Zhou 2011, Li and Li 2012, Liu, Qin et al. 2012). The functional divergence between ASXL1 and ASXL2 is due to the presence of CBX5 (HP1 $1 \alpha$ )-binding PxVxL motif in ASXL1 and ASXL3, but absent in ASXL2 (Lee, Cho et al. 2010) (Fig.5). 


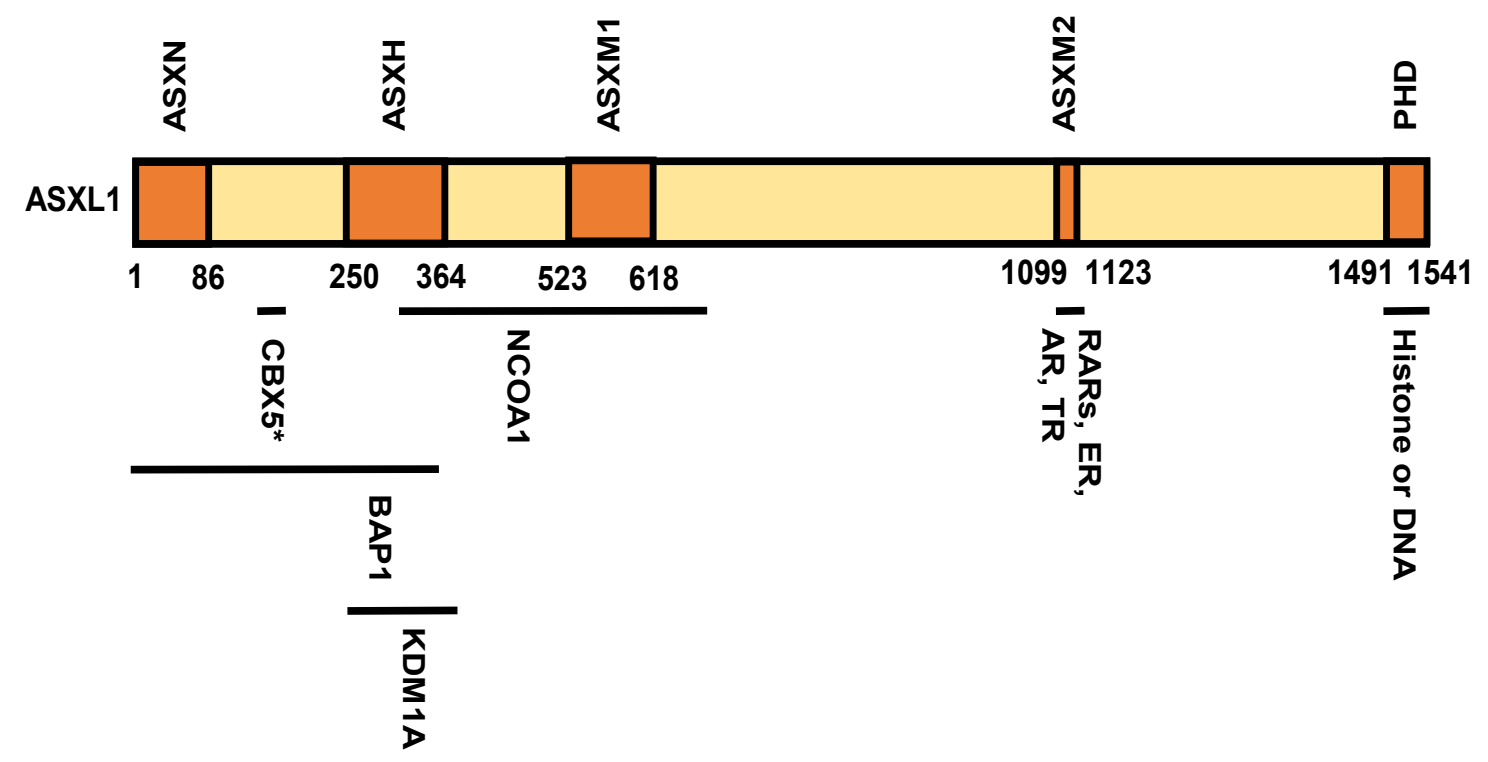

Figure. 5: Schematic representation of ASXL1 protein structure. Human ASXL1 encodes a 1541 amino acid protein. The ASXN and PHD domains bind to modified histones or DNA. The ASXH, ASXM1 and ASXM2 domains are binding modules for BAP1, LSD1 (KDM1A), NCOA1 and NHRs. CBX5 * indicates that CBX5 binds to ASXL1, but not to ASXL2 (modified from Katoh 2013).

ASXL1 contains a number of putative motifs, such as nuclear localization sequences (NLSs), PEST (proline $(P)$, glutamate $(E)$, serine $(S)$ and threonine $(T)$ ) sequences , cyclin interaction substrate recognition sites and phosphorylation sites for cyclindependent kinases (CDKs) (Fisher, Randazzo et al. 2006).

\subsubsection{Functions of ASXL1}

ASXL family members are scaffolding proteins that recruit epigenetic regulators and transcription factors to specific genomic loci with specific histone modifications. ASXL proteins have divergent functions in developing tissues. ASXL1 is highly expressed in the developing and adult hematopoietic cells and depletion of ASXL1 leads to misregulation of hematopoietic progenitor proliferation and differentiation (Fisher, Pineault et al. 2010, Abdel-Wahab, Gao et al. 2013, Wang, Li et al. 2014). ASXL1 is essential for the regulation of Hox gene expression during embryogenesis 
in mice (Fisher, Lee et al. 2010). ASXL1 is required for the maturation of lung and development of eye and cardiovascular system. ASXL1 interacts with polycomb proteins and transcription activators and repressors and regulate epigenetic marks and transcription (Cho, Kim et al. 2006, Boultwood, Perry et al. 2010, Scheuermann, de Ayala Alonso et al. 2010). ASXL1 is important for normal and myeloid differentiation. Both $A S X L 1$ and $A S X L 2$ are essential for the anteroposterior patterning of the mesoderm and homeotic transformation of the axial skeleton. ASXL1 and its paralog ASXL2 play an important role in the regulation of peroxisome proliferator-activated receptors (PPARs) and play opposite roles in adipocyte differentiation. ASXL2 promotes adipocyte differentiation whereas ASXL1 blocks in mouse 3T3-L1 cells (Park, Yoon et al. 2011). It was reported that loss or deletion of ASXL1 in osteoblasts and their progenitors leads to dramatic bone loss and decreased a number of bone marrow stromal cells (BMSCs) (Zhang, Xing et al. 2016).

\subsubsection{ASXL1 and interacting proteins}

ASXL proteins are known to be the members of Enhancer of Trithorax and Polycomb (ETP) group proteins and are activators and repressors of gene transcription, depending on their interacting partners and cellular context. ASXL1 along with BAP1 is a part of the Polycomb repressive deubiquitinase complex (PR-DUB) which deubiquitinates monoubiquitinated H2AK119 mark generated by PRC1 complex and is an important Polycomb Repressor group protein, responsible for repressing genes controlling cell differentiation. Both ASXL1 \& 2 regulate the de-ubiquitinating activity of BAP1 (Scheuermann, de Ayala Alonso et al. 2010, Sahtoe, van Dijk et al. 2016). ASXL1 is associated with the components of the polycomb repressive complex 2 
(PRC2), such as SUZ12 and EZH2, which increases the repressive mark, histone H3K27 methylation. Inhibition or loss of ASXL1 function leads to a decrease in H3K27me3 levels (Lee, Cho et al. 2010, Sugimoto, Muramatsu et al. 2010). ASXL1 also interacts with $\mathrm{HP} 1 \alpha / \mathrm{CBX} 5$, a component of the heterochromatin repressive complex through PxVxL motif and regulates the activity of LSD1 (Lysine-Specific Demethylase 1) (Lee, Cho et al. 2010, Beisel and Paro 2011). Apart from BAP1, ASXL1 is also known to interact with the UTX (Ubiquitously Transcribed Tetratricopeptide Repeat, X chromosome) and LSD1, histone demethylases as well as the MLL (Mixed-Lineage Leukemia) and EZH2 (Enhancer of Zeste Homologue 2) histone methyltransferases (Gelsi-Boyer, Brecqueville et al. 2012).

\subsubsection{ASXL1 in cancer}

Mutations of ASXL1 are associated with several cancers. ASXL family members have dual functions, as they act either as tumor suppressors or as oncogenes in a context-dependent manner. Truncation mutations in the ASXL1 gene are frequently observed in myeloid malignancies, whereas amplification, translocation or point mutations of the ASXL1 gene have been observed in other types of human cancers (Gelsi-Boyer, Trouplin et al. 2009). Nonsense point mutations or frame-shift mutations of ASXL1 occur in hematological malignancies such as myelodysplastic syndrome (MDS), myeloproliferative neoplasms (MPN), acute myeloid leukemia (AML) and chronic lymphocytic leukemia (CLL) (Carbuccia, Murati et al. 2009, GelsiBoyer, Trouplin et al. 2009, Quesada, Conde et al. 2012). ASXL1 mutations lead to aggressive disease and the patients have poor over survival rate. Whole-exome sequencing analyses revealed that ASXL1 is mutated in $2.9 \%$ of CLL cases (Quesada, Conde et al. 2012), whereas meta-analyses revealed that ASXL1 is 
mutated in $45.3 \%$ of $C M M L$ cases, $30 \%$ of secondary $A M L$ cases, $16.2 \%$ of MDS cases, $34.5 \%$ of MPN cases, and $6.5 \%$ of de novo AML cases (Gelsi-Boyer, Brecqueville et al. 2012). ASXL1 is most frequently mutated in colorectal cancer (CRC) cell lines with microsatellite instability (MSI) (Williams, Bird et al. 2010). ASXL1 is also mutated in several other cancers such as castration-resistant prostate cancer, breast cancer, liver cancer and head and neck squamous cell carcinoma ( $\mathrm{Li}$, Zhao et al. 2011, Stransky, Egloff et al. 2011, Grasso, Wu et al. 2012). ASXL1 is amplified and overexpressed in $5.1 \%$ of cervical cancers (Scotto, Narayan et al. 2008). ASXL1 mutations are associated with adverse outcome in leukemia patients (Katoh 2013, Aumann and Abdel-Wahab 2014). Mutations in ASXL1 leads to depletion of ASXL1 protein or truncated protein expression. The truncated proteins lead to gain-of-function as they retain the $\mathrm{N}$-terminal BAP1 interacting domain and leads to enhanced H2AK119Ub deubiquitinating activity.(Scheuermann, de Ayala Alonso et al. 2010, Balasubramani, Larjo et al. 2015)

\subsection{Aim of the project}

ASXL family members are known to be mutated in several cancers and their role in cancers was poorly characterized. Many reports showed that ASXL proteins have epigenetic regulatory functions by recognizing and replacing several histone modifications by interacting with demethylases, methyltransferases and deubiquitinases. In this study our aim is to elucidate the role of ASXL1 during tumor progression and metastasis, especially in epithelial-to-mesenchymal transition (EMT) and decipher its epigenetic mode of action. To understand the ASXL1 mediated effects on tumorigenesis, two approaches were followed. In our first approach, ASXL1 was either depleted or ectopically overexpressed in normal mammary cells 
and breast cancer cells. In the other approach, RNA-seq and ChIP-seq analyses were performed to elucidate its molecular mechanism of action. 


\section{Materials}

\subsection{Technical equipment}

\begin{tabular}{|c|c|}
\hline EQUIPMENT & COMPANY \\
\hline 2100 Bioanalyzer & Agilent Technology, Santa Clara, USA \\
\hline 5100 Cryo $1^{\circ} \mathrm{C}$ Freezing Container & Thermo Fisher Scientific, Waltham, USA \\
\hline Agarose gel chamber & Harnischmacher Labortechnik, Kassel \\
\hline Balance & Sartorius AG, Goettingen \\
\hline Bandelin Sonoplus Sonicator & Bandelin electr. GmbH \& Co. KG, Berlin \\
\hline Biological Safety Cabinet " Hera Safe " & Thermo Fisher Scientific, Waltham, USA \\
\hline Bioruptor $^{\circledR}$ Plus Sonicator & Diagenode SA, Liege, Belgium \\
\hline C1000 TM Thermal Cycler & Bio-Rad Laboratories GmbH, Muenchen \\
\hline Centrifuge (Megafuge 1.OR) & Thermo Fisher Scientific, Waltham, USA \\
\hline Centrifuge $4^{\circ} \mathrm{C}(5417 \mathrm{R})$ & Eppendorf AG, Hamburg \\
\hline Centrifuge $4^{\circ} \mathrm{C}$ (Fesco 21) & Thermo Fisher Scientific, Waltham, USA \\
\hline CFX96 ${ }^{\text {TM }}$ Optical Reaction Module & Bio-Rad Laboratories GmbH, Muenchen \\
\hline Confocal microscope LSM510 META & Leica, Germany \\
\hline Counting chamber (Neubauer) & Brand GmbH \& Co. KG, Wertheim \\
\hline DynaMag $^{\mathrm{TM}} 2$ & Life Technology, Carlsbad, USA \\
\hline DynaMag $^{\text {TM }} 96$ side & Life Technology, Carlsbad, USA \\
\hline Eclipse TS100 & Nikon, Tokyo, Japan \\
\hline Electrophoresis \& Electro transfer Unit & Bio-Rad Laboratories GmbH, Muenchen \\
\hline Freezer $-150^{\circ} \mathrm{C}$ (MDF-C2156VAN) & Panasonic, Kadoma, Japan \\
\hline Freezer $-20^{\circ} \mathrm{C}$ & Liebherr $\mathrm{GmbH}$, Biberach \\
\hline Freezer $-80^{\circ} \mathrm{C}$ "Hera freeze" & Thermo Fisher Scientific, Waltham, USA \\
\hline Gel Imager Gel iX Imager & Intas Science Imaging $\mathrm{GmbH}$, Goettingen \\
\hline HERA cell 150i CO2 Incubator & Thermo Scientific, Waltham, USA \\
\hline Incubator (bacteria culture) & Infors AG, Bottmingen \\
\hline Incubator (bacteria) & Memmert GmbH \& Co. KG, Schwabach \\
\hline Incubator (cell culture) "Hera cell 150“ & Thermo Fisher Scientific, Waltham, USA \\
\hline Inverse Microscope "Axiovert 40 CFL" & Carl Zeiss Microlmaging GmbH, Goettingen \\
\hline Isotemp ${ }^{\circledR}$ water bath & Thermo Fischer Scientific, Waltham, USA \\
\hline Magnet stirrer "MR3001" & Heidolph GmbH \& Co. KG, Schwabach \\
\hline Microwave & Clatronic International GmbH, Kempen \\
\hline Mini Trans-Blot ${ }^{\mathrm{TM}}$ Cell & Bio-Rad Laboratories, Hercules, USA \\
\hline
\end{tabular}




\begin{tabular}{|c|c|}
\hline Mini-PROTEAN Tetra Cell & Bio-Rad Laboratories, Hercules, USA \\
\hline Mr.Frosty ${ }^{\circledR}$ Cryo Freezer & Thermo Fischer Scientific, Waltham, USA \\
\hline Nano Drop ${ }^{\circledR}$ ND-1000 & Peqlab Biotechnology $\mathrm{GmbH}$, Erlangen \\
\hline pH meter inoLab ${ }^{\circledR}$ & WTW GmbH, Weilheim \\
\hline Pipette Aid $^{\circledR}$ portable XP & Drummond Scientific Co., Broomall, USA \\
\hline Pipettes "Research" Series & Eppendorf AG, Hamburg \\
\hline Power supply "Power Pack P25T" & Biometra GmbH, Goettingen \\
\hline PowerPac ${ }^{\top \mathrm{M}}$ Basic Power Supply & Bio-Rad Laboratories, Hercules, USA \\
\hline PowerPac $^{\mathrm{TM}} \mathrm{HC}$ Power Supply & Bio-Rad Laboratories, Hercules, USA \\
\hline Qubit $\circledast 2.0$ Fluorometer & Invitrogen $\mathrm{GmbH}$, Karlsruhe \\
\hline Refrigerator & Liebherr GmbH, Biberach \\
\hline Repeat pipette & Gilson Inc; Middleton, USA \\
\hline Scanner Epson V700 Photo & Seiko Epson, Suwa, Japan \\
\hline ScanScope XT & LMS Co., Ltd., Tokyo, Japan \\
\hline Shaker "Rocky" & Schuett Labortechnik GmbH, Goettingen \\
\hline Table centrifuge (VWR Mini Star) & VWR International, LLC, South Korea \\
\hline Test tube rotator & Schuett Labortechnik GmbH, Goettingen \\
\hline Thermo mixer $\mathrm{C}$ & Eppendorf AG, Wessling-Berzdorf \\
\hline Ultrapure Water System "Aquintus" & Scientific Industries, Inc., Bohemia, USA \\
\hline Vacuum pump (BVC Control) & Vacuubrand $\mathrm{GmbH}$ and Co. KG, Germany \\
\hline Vortex-Genie 2 & Electro Scientific Industr. Inc; Portland, USA \\
\hline Water bath "TW 20" & noLab® WTW GmbH, Weilheim \\
\hline X- Ray Cassettes & Rego X-ray GmbH, Augsburg \\
\hline
\end{tabular}

\subsection{Consumable materials}

\begin{tabular}{|l|l|}
\hline \multicolumn{1}{|c|}{ MATERIAL } & \multicolumn{1}{c|}{ COMPANY } \\
\hline 96 Multiply ${ }^{\circledR}$ PCR plate white & Sarstedt AG \& Co., Nuembrecht \\
\hline $\begin{array}{l}\text { 96-well Multiplate PCR plate white } \\
\text { (Pena-Llopis, Vega-Rubin-de-Celis et } \\
\text { al.) }\end{array}$ & Bio-Rad Laboratories GmbH, Muenchen \\
\hline Cell scraper $(16 \mathrm{~cm}, 25 \mathrm{~cm})$ & Sarstedt AG \& Co., Nuembrecht \\
\hline Cellstar 6- and $12-$ well cell culture plate & Greiner Bio-One $\mathrm{GmbH}$, Frickenhausen \\
\hline Cellstar PP-tube 15 and $50 \mathrm{ml}$ & Greiner Bio-One $\mathrm{GmbH}$, Frickenhausen \\
\hline Cellstar tissue culture dish $100 \times 20 \mathrm{~mm}$ & Greiner Bio-One $\mathrm{GmbH}$, Frickenhausen \\
\hline
\end{tabular}




\begin{tabular}{|c|c|}
\hline Cellstar tissue culture dish $145 \times 20 \mathrm{~mm}$ & Greiner Bio-One GmbH, Frickenhausen \\
\hline Cryo Tube $^{\mathrm{TM}}$ Vial $(1.8 \mathrm{ml})$ & Thermo Fisher Scientific, Waltham, USA \\
\hline DNA loBind Tube (0.5 and $1.5 \mathrm{ml})$ & Eppendorf AG, Wessling-Berzdorf \\
\hline Falcon ${ }^{\circledR}$ assay plate, 96 well & VWR Int., LLC, West Chester, USA \\
\hline Gel blotting paper (Whatman paper) & Sartorius AG, Goettingen \\
\hline Glass coverslips (18 mm) & Gebr. Rettberg GmbH, Goettingen \\
\hline Hybond ${ }^{\mathrm{TM}}$-PVDF Transfer Membrane & GE Healthcare Europe GmbH, Muenchen \\
\hline Microtube $0,5 \mathrm{ml}, 1.5 \mathrm{ml}, 2 \mathrm{ml}$ & Sarstedt AG \& Co., Nuembrecht \\
\hline Microtube $1.5 \mathrm{ml}$, conical & VWR International GmbH, Darmstadt \\
\hline $\begin{array}{l}\text { NORM-JECT Syringes of different } \\
\text { volume }\end{array}$ & Henke Sass Wolf $\mathrm{GmbH}$, Tuttlingen \\
\hline Parafilm ${ }^{\circledR}$ "M" & $\begin{array}{l}\text { Pechiney Plastic Packaging, Chicago, } \\
\text { USA }\end{array}$ \\
\hline PCR plate white (96-well Multiplate $\left.{ }^{\circledR}\right)$ & Bio-Rad Laboratories GmbH, Muenchen \\
\hline PET track-etched cell culture inserts & BD Bioscience, Franklin Lakes, NJ, USA \\
\hline Petri dish $92 \times 16 \mathrm{~mm}$ & Sarstedt AG \& Co., Nuembrecht \\
\hline Pipette filter tips & Sartorius AG, Goettingen \\
\hline Pipette tips & Greiner Bio-One GmbH, Frickenhausen \\
\hline $\begin{array}{l}\text { Protan }^{\circledR} \text { Nitrocellulose transfer } \\
\text { membrane }\end{array}$ & Whatman GmbH, Dassel \\
\hline Shandon Coverplate & Thermo Fisher Scientific, Waltham, USA \\
\hline Syringe filter, Ca-membrane & Sartorius AG, Goettingen \\
\hline Tissue microarrays US & Biomax,Inc., Rockville, MD, USA \\
\hline Ultralow attachment plates & Corning Life sciences, NY, USA \\
\hline X-ray films "Super RX" & Fujifilm Corp., Tokyo, Japan \\
\hline
\end{tabular}

\subsection{Chemicals}

\begin{tabular}{|l|l|}
\hline \multicolumn{1}{|c|}{ CHEMICAL } & \multicolumn{1}{c|}{ COMPANY } \\
\hline Acetic acid & Carl Roth GmbH \& Co. KG, Karlsruhe \\
\hline Adefodur WB developing concentrate & Adefo-Chemie GmbH, Dietzenbach \\
\hline Adefodur WB fixing concentrate & Adefo-Chemie GmbH, Dietzenbach \\
\hline Agar & USB Corporation, Cleveland, USA \\
\hline Agarose & Biozym Scientific GmbH, Oldendorf \\
\hline Agencount ${ }^{\circledR}$ AMPure ${ }^{\circledR}$ XP Beads & Beckman Coulter Inc. Brea USA \\
\hline Albumin Fraction V (BSA) & Carl Roth GmbH \& Co. KG, Karlsruhe \\
\hline
\end{tabular}




\begin{tabular}{|c|c|}
\hline Ammonium persulfate (APS) & Carl Roth GmbH \& Co. KG, Karlsruhe \\
\hline Ammonium sulfate $(\mathrm{NH} 4)_{2} \mathrm{SO}_{4}$ & Carl Roth GmbH \& Co. KG, Karlsruhe \\
\hline Ampicillin & AppliChem GmbH, Darmstadt \\
\hline Aprotinin & Carl Roth GmbH \& Co. KG, Karlsruhe \\
\hline Bovine Serum Albumin (BSA) & Carl Roth GmbH \& Co. KG, Karlsruhe \\
\hline Bromophenol blue & Sigma-Aldrich Co., St. Louis, USA \\
\hline Calcium Chloride $\left(\mathrm{CaCl}_{2}\right)$ & Carl Roth GmbH \& Co. KG, Karlsruhe \\
\hline Chloroform & Carl Roth GmbH \& Co. KG, Karlsruhe \\
\hline Cholera Toxin & Sigma-Aldrich Co., St. Louis, USA \\
\hline Colorless co-precipitant & Bioline, Luckenwalde \\
\hline Co-precipitant Pink & Sigma-Aldrich Co., St. Louis, USA \\
\hline Crystal violet & Carl Roth GmbH \& Co. KG, Karlsruhe \\
\hline DePeX mounting media & VWR International GmbH \\
\hline Diethylpyrocarbonate (DEPC) & Carl Roth GmbH \& Co. KG, Karlsruhe \\
\hline Dimethyl sulfoxide (DMSO) & AppliChem GmbH, Darmstadt \\
\hline di-Sodium hydrogen phosphate & Carl Roth GmbH \& Co. KG, Karlsruhe \\
\hline Dithiothreitol (DTT) & GIBCO ${ }^{2}$, Invitrogen $\mathrm{GmbH}$, Darmstadt \\
\hline DMEM & $\mathrm{GIBCO}{ }^{\circledR}$, Invitrogen $\mathrm{GmbH}$, Darmstadt \\
\hline DMEM/F12 & Carl Roth GmbH \& Co. KG, Karlsruhe \\
\hline dNTPs & Th. Geyer GmbH \& Co. KG, Renningen \\
\hline Epidermal Growth Factor (EGF) & Carl Roth GmbH \& Co. KG, Karlsruhe \\
\hline Ethanol absolute & Carl Roth GmbH \& Co. KG, Karlsruhe \\
\hline Ethidium bromide & Sigma-Aldrich Co., St. Louis, USA \\
\hline Ethylene diamine tetraacetic acid (EDTA) & Thermo Scientific HyClone, Logan, USA \\
\hline Fetal Bovine Serum (FBS) & Sigma-Aldrich Co., St. Louis, USA \\
\hline Formaldehyde & Carl Roth GmbH \& Co. KG, Karlsruhe \\
\hline Glycerol & Sigma-Aldrich Co., St. Louis, USA \\
\hline Glycine & Carl Roth GmbH \& Co. KG, Karlsruhe \\
\hline Horse Serum & Sigma-Aldrich Co., St. Louis, USA \\
\hline Hydrochloric acid $(\mathrm{HCl})$ & Carl Roth GmbH \& Co. KG, Karlsruhe \\
\hline Hydrocortisone & Sigma-Aldrich Co., St. Louis, USA \\
\hline Immobilon ${ }^{\mathrm{TM}}$ Western HRP substrate & Merck,Millipore KGaA, Darmstadt \\
\hline Insulin & Sigma-Aldrich Co., St. Louis, USA \\
\hline lodacetamide & Sigma-Aldrich Co., St. Louis, USA \\
\hline Isopropanol & Carl Roth GmbH \& Co. KG, Karlsruhe \\
\hline
\end{tabular}




\begin{tabular}{|c|c|}
\hline Leupeptin & Carl Roth GmbH \& Co. KG, Karlsruhe \\
\hline Linear Acrylamide & $\begin{array}{l}\text { Thermo Fischer Scientific, Waltham, } \\
\text { USA }\end{array}$ \\
\hline Lithium chloride (LiCl), 8M & Sigma-Aldrich Co., St. Louis, USA \\
\hline Magnesium chloride $\left(\mathrm{MgCl}_{2}\right)$ & Carl Roth GmbH \& Co. KG, Karlsruhe \\
\hline Methanol & M. Baker B.V., Deventer, Netherlands \\
\hline Monopotassium phosphate & Carl Roth GmbH \& Co. KG, Karlsruhe \\
\hline N-ethylmaleimide (NEM) & Sigma-Aldrich Co., St. Louis, USA \\
\hline Nickel chloride $\left(\mathrm{NiCl}_{2}\right)$ & Sigma-Aldrich Co., St. Louis, USA \\
\hline Nonidet $^{\mathrm{TM}}$ P40 (NP-40) & Sigma-Aldrich Co., St. Louis, USA \\
\hline Opti-MEM & GIBCO $®$, Invitrogen $\mathrm{GmbH}$, Darmstadt \\
\hline PBS tablets & GIBCO $®$, Invitrogen $\mathrm{GmbH}$, Darmstadt \\
\hline Pefabloc SC Protease Inhibitor & Carl Roth GmbH \& Co. KG, Karlsruhe \\
\hline Penicillin-Streptomycin solution & Sigma-Aldrich Co., St. Louis, USA \\
\hline Peptone & Carl Roth GmbH \& Co. KG, Karlsruhe \\
\hline Potassium acetate & Carl Roth GmbH \& Co. KG, Karlsruhe \\
\hline Potassium chloride $(\mathrm{KCl})$ & AppliChem GmbH, Darmstadt \\
\hline Potassium dihydrogen phosphate & Carl Roth GmbH \& Co. KG, Karlsruhe \\
\hline Protein-A Sepharose CL-4B & GE Healthcare, Uppsala, Sweden \\
\hline Protein-G Sepharose 4 Fast Flow & GE Healthcare, Uppsala, Sweden \\
\hline RNAiMAX & $\begin{array}{l}\text { New England Biolabs, Frankfurt am } \\
\text { Main }\end{array}$ \\
\hline RNase inhibitor & Invitrogen $\mathrm{GmbH}$, Karlsruhe \\
\hline Roti®-Phenol & Carl Roth GmbH \& Co. KG, Karlsruhe \\
\hline Rotiphorese ${ }^{\circledR}$ Gel 30 & Carl Roth GmbH \& Co. KG, Karlsruhe \\
\hline Rotipuran ${ }^{\circledR}$ Chloroform & Carl Roth GmbH \& Co. KG, Karlsruhe \\
\hline Rotipuran $^{\circledR}$ isoamylalcohol & Carl Roth GmbH \& Co. KG, Karlsruhe \\
\hline Sepharose $^{\mathrm{TM}} \mathrm{CL}-4 \mathrm{~B}$ & GE Healthcare, Uppsala, Sweden \\
\hline Skim milk powder & Carl Roth GmbH \& Co. KG, Karlsruhe \\
\hline Sodium acetate & Carl Roth GmbH \& Co. KG, Karlsruhe \\
\hline Sodium azide & AppliChem GmbH, Darmstadt \\
\hline Sodium chloride $(\mathrm{NaCl})$ & Carl Roth GmbH \& Co. KG, Karlsruhe \\
\hline Sodium deoxycholate & AppliChem GmbH, Darmstadt \\
\hline Sodium dodecylsulfate (SDS) & Carl Roth GmbH \& Co. KG, Karlsruhe \\
\hline Sodium Fluoride (NaF) & AppliChem GmbH, Darmstadt \\
\hline
\end{tabular}




\begin{tabular}{|c|c|}
\hline Sodium hydroxide $(\mathrm{NaOH})$ & Carl Roth GmbH \& Co. KG, Karlsruhe \\
\hline Sodium pyruvate (Na-Pyr) & USB Corporation, Cleveland, USA \\
\hline ß-Glycerolphosphate (BGP) & Carl Roth GmbH \& Co. KG, Karlsruhe \\
\hline SYBR Green I & Carl Roth GmbH \& Co. KG, Karlsruhe \\
\hline TEMED & GIBCO $^{\circledR}$, Invitrogen $\mathrm{GmbH}$, Darmstadt \\
\hline Tris & Carl Roth GmbH \& Co. KG, Karlsruhe \\
\hline Triton X-100 & Carl Roth GmbH \& Co. KG, Karlsruhe \\
\hline TRIzol $^{\circledR}$ Reagent & AppliChem GmbH, Darmstadt \\
\hline Trypsin-EDTA (0.05\%) & Invitrogen $\mathrm{GmbH}$, Karlsruhe \\
\hline Tween-20 & GIBCO $\AA$, Invitrogen $\mathrm{GmbH}$, Darmstadt \\
\hline Xylene & AppliChem GmbH, Darmstadt \\
\hline Yeast extract & USB Corporation, Cleveland, USA \\
\hline$\alpha, \alpha$-Trehalose Dihydrate & Carl Roth GmbH \& Co. KG, Karlsruhe \\
\hline
\end{tabular}

\subsection{Kits and Reagents}

\begin{tabular}{|l|l|}
\hline \multicolumn{1}{|c|}{ KIT and REAGENT } & \multicolumn{1}{c|}{ COMPANY } \\
\hline Agilent High Sensitivity DNA Kit & Agilent Technology, Santa Clara, USA \\
\hline Bioanalyzer DNA High sensitivity kit & Agilent Technology, Santa Clara, USA \\
\hline $\begin{array}{l}\text { Immobilon Western Chemiluminescent } \\
\text { HRP Substrate }\end{array}$ & Millipore, Billerica, USA \\
\hline ISOLATE II PCR and Gel Kit & Bioline USA Inc; Taunton, USA \\
\hline LipofectamineTM 2000 & Life technology, Carlsbad, USA \\
\hline LipofectamineTM RNAiMAX & Life technology, Carlsbad, USA \\
\hline Microplex Library PreparationTM V2 Kit & Diagenode SA, Liege, Belgium \\
\hline Microplex Library PreparationTM Kit & Diagenode SA, Liege, Belgium \\
\hline NEBNEXT® UltraTM Library Prep Kit & New England Biolabs, Ipswich, USA \\
\hline NucleoBond® Xtra Midi & Macherey-Nagel Inc; Bethlehem, USA \\
\hline PageRuler ${ }^{\top M}$ Prestained Protein Ladder & Fermentas GmbH, St. Leon--Rot \\
\hline QIAprep Spin Miniprep Kit & Qiagen GmbH, Hilden \\
\hline Qubit dsDNA HS assay & Life technology, Carlsbad, USA \\
\hline SuperSigmal囚 West Femto Maximum & $\begin{array}{l}\text { Thermo Fisher Scientific, Waltham, } \\
\text { USA }\end{array}$ \\
\hline SuperSignal® West Dura & $\begin{array}{l}\text { Thermo Fisher Scientific, Waltham, } \\
\text { USA }\end{array}$ \\
\hline
\end{tabular}




\subsection{Nucleic acids}

\subsubsection{Vectors and expression constructs}

\begin{tabular}{|l|l|}
\hline \multicolumn{1}{|c|}{ Name } & \multicolumn{1}{c|}{ Reference } \\
\hline pCMV6-Flag-ASXL1 & (Abdel-Wahab, Adli et al. 2012) \\
\hline pSG5-HA-P2A-ERT2-Hygromycin & This study \\
\hline pSG5-HA-P2A-ASXL1-Hygromycin & This study \\
\hline
\end{tabular}

\subsection{2 siRNA Oligonucleotides}

For transfections, the individual siRNAs (- \#4) against the respective genes were pooled in a 1:1:1:1 ratio.

\begin{tabular}{|l|l|l|l|}
\hline \multicolumn{1}{|c|}{ siRNA } & Target sequence 5'-3' direction & \multicolumn{1}{c|}{ Source } & \multicolumn{1}{c|}{ Cat. No. } \\
\hline siASXL1\#1 & GAUCGUCAGUCCUUUCGUA & Dharmacon & D-012856-01 \\
\hline siASXL1\#2 & GGAUUCAACUUUCACGUAU & Dharmacon & D-012856-02 \\
\hline siASXL1\#3 & GCAGUGCACUAAAUAACGA & Dharmacon & D-012856-03 \\
\hline siASXL1\#4 & GAAAGUGUACGUCAGAUCU & Dharmacon & D-012856-04 \\
\hline $\begin{array}{l}\text { Non-targetting } \\
\text { siRNA-5 }\end{array}$ & - & Dharmacon & $\begin{array}{l}\text { D-001210-05- } \\
05\end{array}$ \\
\hline $\begin{array}{l}\text { SiPTEN } \\
\text { SMARTpool }\end{array}$ & - & Dharmacon & $\begin{array}{l}\text { L-003023-00- } \\
0002\end{array}$ \\
\hline
\end{tabular}

\subsubsection{Cloning primers}

\begin{tabular}{|l|l|c|}
\hline \multicolumn{1}{|c|}{ Gene Name } & \multicolumn{1}{|c|}{ Primer Sequence (5' $\mathbf{-} \mathbf{3}^{\prime}$ ) } & Reference \\
\hline ASXL1 F & $\begin{array}{l}\text { GCTGACGCGGCCGCAATGAAGGA } \\
\text { CAAACAGAAGAAG }\end{array}$ & This study \\
\hline ASXL1 R & $\begin{array}{l}\text { GCTGACGCTAGCTCTCACCACAA } \\
\text { GGCACAATACACAG }\end{array}$ & This study \\
\hline
\end{tabular}

\subsubsection{RT PCR primers}

Reverse transcription primers were ordered from Sigma-Aldrich, Germany. 


\subsubsection{Quantitative PCR primers (qPCR)}

Quantitative PCR primers were designed using the NCBI primer designing tool (http://www.ncbi.nlm.nih.gov/tools/primer-blast/).

\begin{tabular}{|l|l|c|}
\hline \multicolumn{1}{|c|}{ Gene Name } & \multicolumn{1}{|c|}{ Primer Sequence (5' - $\mathbf{3}^{\prime}$ ) } & Reference \\
\hline ARID1B F & GCAAGGTGTGAGTGGTTACTG & This study \\
\hline ARID1B R & GGACTGGGACGGCAGATACT & This study \\
\hline ASXL1 F & GGTCCTGTCTCAGTCCCTCA & This study \\
\hline ASXL1 R & ATAACCACGGGGTCAGAGGT & This study \\
\hline CD24 F & GCTCCTACCCACGCAGATTT & This study \\
\hline CD24 R & GAGACCACGAAGAGACTGGC & This study \\
\hline CDH1 F & CTTTGACGCCGAGAGCTACA & This study \\
\hline CDH1 R & AAATTCACTCTGCCCAGGACG & This study \\
\hline CDH2 F & GGGTCATCCCTCCAATCAAC & This study \\
\hline CDH2 R & ACCTGATCCTGACAAGCTCT & This study \\
\hline DLL1 F & GCAAGCGTGACACCAAGTG & This study \\
\hline DLL1 R & AAGTTGAACAGCCCGAGTCC & This study \\
\hline FN1 F & CCCTGGTGTCACAGAGGCTA & This study \\
\hline FN1 R & GAGAGAGAGCTTCTTGTCCTGTC & This study \\
\hline HNRNPK F & ATCCGCCCCTGAACGCCCAT & $\begin{array}{l}\text { (Karpiuk, Najafova } \\
\text { et al. 2012) }\end{array}$ \\
\hline HNRNPK R & ACATACCGCTCGGGGCCACT & $\begin{array}{l}\text { (Karpiuk, Najafova } \\
\text { et al. 2012) }\end{array}$ \\
\hline PTEN F & GTGGCGGAACTTGCAATCCT & This study \\
\hline PTEN R & CGGCTGAGGGAACTCAAAGT & This study \\
\hline TJP3 F & CAGAGCATGGAGGATCGTGG & This study \\
\hline TJP3 R & TCAGGTTCTGGAATGGCACG & This study \\
\hline VIM F & GGCTCGTCACCTTCGTGAAT & This study \\
\hline VIM R & CAGAGAAATCCTGCTCTCCTCG & This study \\
\hline
\end{tabular}

\subsubsection{ChIP Primers}

\begin{tabular}{|l|l|l|}
\hline \multicolumn{1}{|c|}{ Gene Name } & \multicolumn{1}{c|}{ Primer Sequence $\left(\mathbf{5}^{\prime} \mathbf{-} \mathbf{3}^{\prime}\right)$} & Reference \\
\hline PTEN TSS F & AACCCTCCTAGGTCTCCTCG & This study \\
\hline PTEN TSS R & TCGGTTCTCAGAGACCACCT & This study \\
\hline
\end{tabular}




\begin{tabular}{|l|l|l|}
\hline TJP3 TSS F & GGCCGATTGACTGTTTCCAG & This study \\
\hline TJP3 TSS R & GTTCTGGGGTCCTGGGTC & This study \\
\hline ARID1B TSS F & GGGAGTAATGCGAGCGAAGT & This study \\
\hline ARID1B TSS R & TCCTCTCTAGCCCGGATATGG & This study \\
\hline
\end{tabular}

\subsection{Proteins, enzymes and standards}

\subsubsection{Antibodies}

\subsubsection{Primary antibodies}

Primary antibodies for western blot, ChIP and immunofluorescence were used in the respective dilutions and concentrations. The antibody dilutions for immunoblot analysis were supplemented with $0.01 \%$ sodium azide.

\begin{tabular}{|c|c|c|c|c|c|c|}
\hline Antibody & Source & Cat. No. & Clone & WB & IF & ChIP \\
\hline ASXL1 & Santacruz & sc-98302 & $\mathrm{H}-105$ & $1: 250$ & & $1 \mu \mathrm{g}$ \\
\hline E-Cadherin & Cell Signalling & 3195 & 24E10 & $1: 1000$ & $1: 200$ & \\
\hline N-Cadherin & Cell Signalling & $\# 13116$ & $\mathrm{D} 4 \mathrm{R} 1 \mathrm{H}$ & $1: 1000$ & & \\
\hline CD24 & Hans Peter & - & SWA11 & $1: 2$ & & \\
\hline Vimentin & Santacruz & sc-6260 & V9 & $1: 1000$ & $1: 200$ & \\
\hline $\mathrm{ZO}-1$ & Cell Signalling & 8193 & D7D12 & $1: 1000$ & & \\
\hline ZEB1 & Sigma & HPA027524 & - & $1: 500$ & & \\
\hline BAP1 & Santacruz & sc-28383 & C-4 & $1: 500$ & & $1 \mu g$ \\
\hline AKT & Cell Signalling & 9272 & & $1: 1000$ & & \\
\hline P-AKT & Cell Signalling & 4060 & & $1: 1000$ & & \\
\hline $\mathrm{H} 3 \mathrm{~K} 4 \mathrm{me} 3$ & Diagenode & - & $\begin{array}{l}\text { pAb- } \\
003-050\end{array}$ & $1: 1000$ & & $1 \mu \mathrm{g}$ \\
\hline H3K27me3 & Diagenode & - & $\begin{array}{l}\text { pAb- } \\
195-050\end{array}$ & $1: 1000$ & & \\
\hline H2Aub1 & Diagenode & - & & $1: 1000$ & & \\
\hline H2Bub1 & Hybridoma & - & 7B4 & $1: 10$ & & \\
\hline $\mathrm{H} 2 \mathrm{~B}$ & Cell Signalling & 2934 & $53 \mathrm{H} 3$ & $1: 5000$ & & \\
\hline HSC-70 & Santacruz & sc-7298 & B-6 & $\begin{array}{l}1: 10,00 \\
0\end{array}$ & & \\
\hline
\end{tabular}




\begin{tabular}{|l|l|c|c|c|c|c|}
\hline $\begin{array}{l}\text { IgG (non- } \\
\text { specific) }\end{array}$ & Abcam & Ab37415 & - & & & $1 \mu \mathrm{g}$ \\
\hline WDR82 & David G.Skalnik & - & - & $1: 500$ & & \\
\hline Cfp1 & David G.Skalnik & - & - & $1: 250$ & & \\
\hline
\end{tabular}

\subsubsection{Secondary antibodies}

\begin{tabular}{|l|l|l|c|c|}
\hline \multicolumn{1}{|c|}{ Antibody } & \multicolumn{1}{|c|}{ Source } & Cat. No. & WB & IF \\
\hline Goat Anti-Mouse IgG-HRP & Santacruz & sc-2005 & $1: 10,000$ & \\
\hline Goat Anti-Rabbit IgG-HRP & Santacruz & sc-2004 & $1: 10,000$ & \\
\hline $\begin{array}{l}\text { Alexa Fluor } \\
\text { Mouse IgG Goat Anti- }\end{array}$ & Life Technologies & A11005 & & $1: 500$ \\
\hline $\begin{array}{l}\text { Alexa Fluor } \\
\text { Rabbit IgG }\end{array}$ & Life Technologies & A11008 & & $1: 500$ \\
\hline
\end{tabular}

\subsubsection{Enzymes}

\begin{tabular}{|l|l|}
\hline \multicolumn{1}{|c|}{ Enzyme } & \multicolumn{1}{c|}{ Company } \\
\hline Phusion Polymerase & New England Biolabs, Frankfurt am Main \\
\hline Proteinase K & Life Technology, Carlsbad, USA \\
\hline Restriction enzymes & New England Biolabs, Frankfurt am Main \\
\hline T4 DNA Ligase & New England Biolabs, Frankfurt am Main \\
\hline Reverse Transcriptase (M-MuLV) & New England Biolabs, Frankfurt am Main \\
\hline RNase A & Qiagen GmbH, Hilden \\
\hline Taq DNA Polymerase & Prime Tech, Minsk, Belarus \\
\hline RNase Inhibitor & New England Biolabs, Frankfurt am Main \\
\hline
\end{tabular}

\subsubsection{Molecular weight standards}

\begin{tabular}{|l|l|}
\hline \multicolumn{1}{|c|}{ Standard } & \multicolumn{1}{c|}{ Company } \\
\hline Gene Ruler ${ }^{\mathrm{TM}}$ DNA-Ladder & Fermentas GmbH, St. Leon-Rot \\
\hline Page Ruler ${ }^{\mathrm{TM}}$ Prestained Protein Ladder & Fermentas $\mathrm{GmbH}$, St. Leon-Rot \\
\hline
\end{tabular}




\subsection{Buffers and Solutions}

\subsubsection{ChIP Buffers}

Nelson Buffer (modified)

\begin{tabular}{|l|l|}
\hline \multicolumn{1}{|c|}{ Component } & Final concentration \\
\hline $\mathrm{NaCl}$ & $150 \mathrm{mM}$ \\
\hline EDTA $(\mathrm{pH}$ 8.0) & $20 \mathrm{mM}$ \\
\hline Tris $(\mathrm{pH} 7.5)$ & $50 \mathrm{mM}$ \\
\hline $\mathrm{NP}-40(\mathrm{v} / \mathrm{v})$ & $0.5 \%$ \\
\hline Triton-X-100 (v/v) & $1 \%$ \\
\hline $\mathrm{NaF}$ & $20 \mathrm{mM}$ \\
\hline
\end{tabular}

Gomes Lysis Buffer

\begin{tabular}{|l|l|}
\hline \multicolumn{1}{|c|}{ Component } & Final concentration \\
\hline $\mathrm{NaCl}$ & $150 \mathrm{mM}$ \\
\hline $\mathrm{NP}-40(\mathrm{v} / \mathrm{v})$ & $1 \%$ \\
\hline Sodium deoxycholate & $0.5 \%$ \\
\hline Tris-HCl $\mathrm{pH} 8.0$ & $50 \mathrm{mM}$ \\
\hline EDTA & $20 \mathrm{mM}$ \\
\hline $\mathrm{NaF}$ & $20 \mathrm{mM}$ \\
\hline SDS & $0.1 \%$ \\
\hline
\end{tabular}

Weinmann Lysis Buffer

\begin{tabular}{|l|l|}
\hline \multicolumn{1}{|c|}{ Component } & Final concentration \\
\hline Tris- $\mathrm{HCl} \mathrm{pH} 8.0$ & $50 \mathrm{mM}$ \\
\hline EDTA & $10 \mathrm{mM}$ \\
\hline SDS $(\mathrm{w} / \mathrm{v})$ & $1 \%$ \\
\hline
\end{tabular}

Gomes Wash Buffer 


\begin{tabular}{|l|l|}
\hline Tris- $\mathrm{HCl} \mathrm{pH} 8$ & $100 \mathrm{mM}$ \\
\hline $\mathrm{LiCl}$ & $500 \mathrm{mM}$ \\
\hline $\mathrm{NP}-40(\mathrm{v} / \mathrm{v})$ & $1 \%$ \\
\hline Sodium deoxycholate $(\mathrm{w} / \mathrm{v})$ & $1 \%$ \\
\hline EDTA & $20 \mathrm{mM}$ \\
\hline $\mathrm{NaF}$ & $20 \mathrm{mM}$ \\
\hline
\end{tabular}

TE Buffer

\begin{tabular}{|l|l|}
\hline \multicolumn{1}{|c|}{ Component } & Final concentration \\
\hline Tris- $\mathrm{HCl} \mathrm{pH} 8.0$ & $10 \mathrm{mM}$ \\
\hline EDTA $\mathrm{pH} 8.0$ & $1 \mathrm{mM}$ \\
\hline
\end{tabular}

\section{Cross-linking buffer}

\begin{tabular}{|l|l|}
\hline \multicolumn{1}{|c|}{ Component } & Final concentration \\
\hline Formaldehyde & $1 \%$ \\
\hline PBS & $1 \mathrm{x}$ \\
\hline
\end{tabular}

Proteinase inhibitors

\begin{tabular}{|l|l|l|}
\hline \multicolumn{1}{|c|}{ Component } & \multicolumn{1}{c|}{$\begin{array}{c}\text { Stock } \\
\text { concentration }\end{array}$} & \multicolumn{1}{c|}{$\begin{array}{c}\text { Final } \\
\text { concentration }\end{array}$} \\
\hline $\mathrm{NiCl}_{2}$ & $1 \mathrm{mM}$ & $1: 1000$ \\
\hline Pefabloc & $1 \mathrm{mM}$ & $1: 100$ \\
\hline IAA & $10 \mu \mathrm{M}$ & $1: 10000$ \\
\hline $\mathrm{BGP}$ & $10 \mathrm{mM}$ & $1: 100$ \\
\hline $\mathrm{NEM}$ & $1 \mathrm{mM}$ & $1: 100$ \\
\hline Aprotinin/Leupeptin & $1 \mathrm{ng} / \mu \mathrm{l}$ & $1: 1000$ \\
\hline
\end{tabular}

\subsubsection{Western blot Buffers}

SDS separating gel (X \%)

\begin{tabular}{|l|l|}
\hline Acrylamide & $\mathrm{X} \%$ \\
\hline Tris-HCl pH 8.8 & $375 \mathrm{mM}$ \\
\hline
\end{tabular}




\begin{tabular}{|l|l|}
\hline SDS $(w / v)$ & $0.1 \%$ \\
\hline APS $(v / v)$ & $0.1 \%$ \\
\hline TEMED & $0.04 \%$ \\
\hline
\end{tabular}

SDS stacking gel (5\%)

\begin{tabular}{|l|l|}
\hline Acrylamide & $5 \%$ \\
\hline Tris-HCl pH 6.8 & $125.5 \mathrm{mM}$ \\
\hline SDS (w/v) & $0.1 \%$ \\
\hline APS (v/v) & $0.1 \%$ \\
\hline TEMED & $0.1 \%$ \\
\hline
\end{tabular}

Transfer buffer

\begin{tabular}{|l|l|}
\hline Western salts (10X) (v/v) & $10 \%$ \\
\hline Methanol (v/v) & $20 \%$ \\
\hline
\end{tabular}

Western salts (10X)

\begin{tabular}{|l|l|}
\hline Glycine & $1.92 \mathrm{M}$ \\
\hline Tris-HCl pH 8.3 & $250 \mathrm{mM}$ \\
\hline SDS & $0.02 \%$ \\
\hline
\end{tabular}

\section{X Laemmli buffer}

\begin{tabular}{|l|l|}
\hline Tris-HCl pH 6.8 & $0.35 \mathrm{M}$ \\
\hline Glycerol & $30 \%$ \\
\hline SDS (w/v) & $10 \%$ \\
\hline DTT & $9.3 \%$ \\
\hline Bromophenol blue & $0.02 \%$ \\
\hline
\end{tabular}

\section{SDS Running Buffer}

\begin{tabular}{|l|l|}
\hline Glycine & $200 \mathrm{mM}$ \\
\hline Tris-HCl & $25 \mathrm{mM}$ \\
\hline
\end{tabular}




\begin{tabular}{|l|l|}
\hline SDS $(w / v)$ & $0.1 \%$ \\
\hline
\end{tabular}

TBS 10X pH 7.6

\begin{tabular}{|l|l|}
\hline Tris- $\mathrm{HCl}$ & $100 \mathrm{mM}$ \\
\hline $\mathrm{NaCl}$ & $150 \mathrm{mM}$ \\
\hline
\end{tabular}

TBS-T

$$
\text { TBS }+0.1 \%(w / v) \text { Tween-20 }
$$

RIPA buffer

\begin{tabular}{|l|l|}
\hline PBS & $1 \mathrm{X}$ \\
\hline NP-40 $(\mathrm{v} / \mathrm{v})$ & $1 \%$ \\
\hline Sodium deoxycholate & $0.5 \%$ \\
\hline SDS $(\mathrm{w} / \mathrm{v})$ & $0.1 \%$ \\
\hline
\end{tabular}

\section{Blocking solution}

\begin{tabular}{|l|l|}
\hline TBST & $1 \mathrm{X}$ \\
\hline Skimmed milk (w/v) & $5 \%$ \\
\hline
\end{tabular}

Proteinase inhibitors

\begin{tabular}{|l|l|l|}
\hline \multicolumn{1}{|c|}{ Component } & \multicolumn{1}{c|}{$\begin{array}{c}\text { Stock } \\
\text { concentration }\end{array}$} & \multicolumn{1}{c|}{$\begin{array}{c}\text { Final } \\
\text { concentration }\end{array}$} \\
\hline Pefabloc & $1 \mathrm{mM}$ & $1: 100$ \\
\hline BGP & $10 \mathrm{mM}$ & $1: 100$ \\
\hline NEM & $1 \mathrm{mM}$ & $1: 100$ \\
\hline Aprotinin/Leupeptin & $1 \mathrm{ng} / \mu \mathrm{l}$ & $1: 1000$ \\
\hline
\end{tabular}

10X PBS (pH 7.4)

\begin{tabular}{|l|l|}
\hline $\mathrm{NaCl}$ & $730 \mathrm{mM}$ \\
\hline $\mathrm{KCl}$ & $27 \mathrm{mM}$ \\
\hline
\end{tabular}




\begin{tabular}{|l|l|}
\hline $\mathrm{NaH}_{2} \mathrm{PO}_{4}{ }^{*} 7 \mathrm{H}_{2} \mathrm{O}$ & $14.3 \mathrm{mM}$ \\
\hline $\mathrm{KH}_{2} \mathrm{PO}_{4}$ & $14.7 \mathrm{mM}$ \\
\hline
\end{tabular}

PBS-T

$$
\text { PBS }+0.1 \%(w / v) \text { Tween-20 }
$$

PCR-Mix 10X

\begin{tabular}{|l|l|}
\hline \multicolumn{1}{|c|}{ Component } & \multicolumn{1}{c|}{ Final concentration } \\
\hline Tris- $\mathrm{HCl} \mathrm{pH} \mathrm{8.8}$ & $750 \mathrm{mM}$ \\
\hline$\left(\mathrm{NH}_{4}\right)_{2} \mathrm{SO}_{4}$ & $200 \mathrm{mM}$ \\
\hline Tween-20 & $0.1 \%$ \\
\hline
\end{tabular}

\section{RT-PCR Master Mix}

\begin{tabular}{|l|l|}
\hline \multicolumn{1}{|c|}{ Component } & \multicolumn{1}{c|}{ Concentration } \\
\hline PCR-Mix & $1 \mathrm{X}$ \\
\hline $\mathrm{MgCl}_{2}$ & $3 \mathrm{mM}$ \\
\hline SYBR Green & $1: 80,000$ \\
\hline dNTPs & $0.2 \mathrm{mM}$ \\
\hline Taq-polymerase & $20 \mathrm{U} / \mathrm{ml}$ \\
\hline Triton X-100 & $0.25 \%$ \\
\hline Trehalose & $300 \mathrm{mM}$ \\
\hline
\end{tabular}

TAE buffer (50X)

\begin{tabular}{|l|l|}
\hline Tris & $2 \mathrm{M}$ \\
\hline Acetic acid & $1 \mathrm{M}$ \\
\hline EDTA & $0.1 \mathrm{M}$ \\
\hline
\end{tabular}

\subsection{Cell culture medium}




\section{LB Agar}

LB medium

$1 \mathrm{X}$

Agar

$1.5 \%$

\section{LB medium}

Peptone

$1 \%$

Yeast extract

$0.5 \%$

$\mathrm{NaCl}$

$86 \mathrm{mM}$

DMEM cell culture medium

DMEM phenol red medium

Fetal bovine serum

$10 \%$

Penicillin

$100 \mathrm{U} / \mathrm{ml}$

Streptomycin

$100 \mu \mathrm{g} / \mathrm{ml}$

\section{DMEM/F12 cell culture medium}

Phenol red-free high-glucose DMEM/F12

Horse serum

$5 \%$

EGF $100 \mu \mathrm{g} / \mathrm{ml}$

Hydrocortisone $1 \mathrm{mg} / \mathrm{ml}$

Cholera toxin $1 \mathrm{mg} / \mathrm{ml}$

Insulin $10 \mathrm{mg} / \mathrm{ml}$

Penicillin $100 \mathrm{U} / \mathrm{ml}$

Streptomycin $100 \mu \mathrm{g} / \mathrm{ml}$

Cell freezing medium

\begin{tabular}{|l|l|}
\hline DMEM & $42 \%$ \\
\hline FBS & $50 \%$ \\
\hline DMSO & $8 \%$ \\
\hline
\end{tabular}

PBS for cell culture

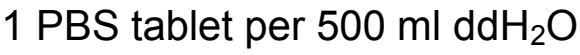




\subsection{Cells}

\subsubsection{Bacterial cells}

Escherichia coli DH10BTM from Invitrogen $\mathrm{GmbH}$, Karlsruhe

\subsubsection{Human Cell lines}

\begin{tabular}{|c|c|c|c|c|}
\hline Cell Line & Species & Tissue Origin & Disease & Source \\
\hline MCF10A & Human & $\begin{array}{l}\text { Mammary } \\
\text { gland/breast }\end{array}$ & $\begin{array}{l}\text { Nontumorigenic } \\
\text { epithelial cell line }\end{array}$ & $\begin{array}{l}\text { ATCC } \\
\text { (CRL- } \\
10317)\end{array}$ \\
\hline MCF12A & Human & $\begin{array}{c}\text { Mammary } \\
\text { gland/breast }\end{array}$ & $\begin{array}{l}\text { Nontumorigenic } \\
\text { epithelial cell line }\end{array}$ & $\begin{array}{l}\text { ATCC } \\
\text { (CRL- } \\
10782)\end{array}$ \\
\hline MDA-MB-231 & Human & $\begin{array}{l}\text { Mammary } \\
\text { gland/breast }\end{array}$ & adenocarcinoma & $\begin{array}{c}\text { ATCC } \\
(\text { HTB-26) }\end{array}$ \\
\hline $\begin{array}{l}\text { MCF10A with stable } \\
\text { pSG5 } \\
\text { overexpression }\end{array}$ & Human & $\begin{array}{c}\text { Mammary } \\
\text { gland/breast }\end{array}$ & $\begin{array}{l}\text { Nontumorigenic } \\
\text { epithelial cell line }\end{array}$ & This study \\
\hline $\begin{array}{l}\text { MCF10A with stable } \\
\text { pSG5-ASXL1 } \\
\text { overexpression }\end{array}$ & Human & $\begin{array}{c}\text { Mammary } \\
\text { gland/breast }\end{array}$ & $\begin{array}{l}\text { Nontumorigenic } \\
\text { epithelial cell line }\end{array}$ & This study \\
\hline $\begin{array}{l}\text { MDA-MB-231 with } \\
\text { stable pSG5 } \\
\text { overexpression }\end{array}$ & Human & $\begin{array}{c}\text { Mammary } \\
\text { gland/breast }\end{array}$ & adenocarcinoma & This study \\
\hline $\begin{array}{l}\text { MDA-MB-231 with } \\
\text { stable pSG5-ASXL1 } \\
\text { overexpression }\end{array}$ & Human & $\begin{array}{c}\text { Mammary } \\
\text { gland/breast }\end{array}$ & adenocarcinoma & This study \\
\hline
\end{tabular}

\section{Co-IP Lysis Buffer}

\begin{tabular}{|l|l|}
\hline \multicolumn{1}{|c|}{ Component } & Final concentration \\
\hline Tris- $\mathrm{HCl} \mathrm{pH} \mathrm{7.1}$ & $5 \mathrm{mM}$ \\
\hline $\mathrm{NaCl}$ & $25 \mathrm{mM}$ \\
\hline Triton $\mathrm{X}-100$ & $0.5 \%$ \\
\hline $\mathrm{NaF}$ & $25 \mathrm{mM}$ \\
\hline $\mathrm{Na}_{3} \mathrm{VO}_{4}$ & $0.5 \mathrm{mM}$ \\
\hline DTT & $0.2 \mathrm{mM}$ \\
\hline
\end{tabular}


2.10 Software and online tools

\begin{tabular}{|l|l|}
\hline \multicolumn{1}{|c|}{ NAME } & \multicolumn{1}{c|}{ SOURCE } \\
\hline useGalaxy & $\underline{\text { https://usegalaxy.org/ }}$ \\
\hline Galaxy Cistrome & $\underline{\text { http://cistrome.org/ap/root }}$ \\
\hline DAVID GO analysis & $\underline{\text { http://david.ncifcrf.gov }}$ \\
\hline Galaxy Deeptools & $\underline{\text { http://deeptools.ie-freiburg.mpg.de/ }}$ \\
\hline Kaplan-Meier plotter & $\underline{\text { http://kmplot.com/analysis/index.php?p=background }}$ \\
\hline Bio-Rad CFX Manager 3.1 & Bio-Rad Laboratories, Hercukes, USA \\
\hline $\begin{array}{l}\text { Integrative Genome } \\
\text { Viewer 2 }\end{array}$ & $\underline{\text { https://www.broadinstitute.org/software/igv/download }}$ \\
\hline $\begin{array}{l}\text { Gene Set Enrichment } \\
\text { Analysis }\end{array}$ & $\underline{\text { http://software.broadinstitute.org/gsea/index.jsp }}$ \\
\hline $\begin{array}{l}\text { Image Lab Version 5.2 } \\
\text { build 14 }\end{array}$ & Bio-Rad Laboratories, Hercukes, USA \\
\hline $\begin{array}{l}\text { R statistical software } \\
\text { Onttp://www.r-project.org }\end{array}$ \\
\hline $\begin{array}{l}\text { Oncomine database } \\
\text { Primer designing } \\
\text { tool/NCBI primer-BLAST }\end{array}$ & $\underline{\text { http://www.oncomine.org/resource/main.html }}$ \\
\hline
\end{tabular}




\section{Methods}

\subsection{Cell culture}

\subsubsection{Cell culture of mammary cells}

MCF10A and MCF12A (human breast epithelial) cells were cultured in phenol redfree Dulbecco's Modified Eagle Medium: Nutrient Mixture F-12 (DMEM/F12) medium supplemented with $5 \%$ horse serum, $0.5 \mu \mathrm{g} / \mathrm{ml}$ hydrocortisone, $10 \mu \mathrm{g} / \mathrm{ml}$ Insulin, 20 $\mathrm{ng} / \mathrm{ml}$ human EGF (epithelial growth factor), $0.1 \mu \mathrm{g} / \mathrm{ml}$ Cholera toxin, $100 \mathrm{units} / \mathrm{ml}$ penicillin and $100 \mathrm{\mu g} / \mathrm{ml}$ streptomycin at $37^{\circ} \mathrm{C}$ under $5 \% \mathrm{CO}_{2}$ atmosphere. MDA-MB231 (human breast cancer, adenocarcinoma) cells were cultured in high-glucose Dulbecco's modified Eagles medium (DMEM) and GlutaMAX supplemented with $10 \%$ fetal bovine serum (FBS), 100 units $/ \mathrm{ml}$ penicillin and $100 \mu \mathrm{g} / \mathrm{ml}$ streptomycin at $37^{\circ} \mathrm{C}$ under $5 \% \mathrm{CO}_{2}$ atmosphere.

\subsubsection{Reverse-transfection with siRNA}

Reverse-siRNA transfections were performed using Lipofectamine ${ }^{T M}$ RNAiMAX according to the manufacturer's instructions. For each 6-well transfection, $30 \mathrm{pmol}$ of respective siRNAs were diluted with $500 \mu$ l of Opti-MEM and $5 \mu \mathrm{l}$ of Lipofectamine ${ }^{\mathrm{TM}}$ RNAiMAX was added and mixed gently. The transfection mixture was then incubated for 20 min at RT. Meanwhile, MCF10A, MCF12A or MDA-MB-231 cells were washed with 1XPBS, trypsinized and diluted in "respective normal medium" without antibiotics. Neubauer counting chamber was used to count the cells and nearly 250,000 cells (MCF10A or MCF12A) or 300,000 cells (MDA-MB-231) from the diluted cells were added to each 6 -well containing the siRNA-Lipofectamine ${ }^{\mathrm{TM}}$ RNAiMAX complexes. After $24 \mathrm{~h}$, the medium was replaced with fresh medium 
containing antibiotics. Cells were harvested after $72 \mathrm{~h}$. For transfections in $10 \mathrm{~cm}$ plates, four times more cell number and transfection reagents were used.

\subsubsection{Plasmid DNA transfection}

A day before transfection, cells were plated in $10 \mathrm{~cm}$ plates to attain approximately $70-80 \%$ confluency on the day of transfection. The cells were washed twice with 1XPBS and the medium was replaced with the respective antibiotic free medium. Plasmid transfection was performed using Lipofectamine ${ }^{\mathrm{TM}} 2000$ according to the manufacturer's instructions. For each transfection, 8-10 $\mu \mathrm{g}$ of plasmid DNA was mixed with $2 \mathrm{ml}$ of Opti-MEM in a reaction tube. In a second tube, $20 \mu \mathrm{l}$ of Lipofectamine $^{\mathrm{TM}} 2000$ was mixed with $2 \mathrm{ml}$ of Opti-MEM and incubated for $5 \mathrm{~min}$ at RT. After combining the contents of both tubes and mixing gently by inverting, the samples were incubated for another $20 \mathrm{~min}$ at RT. The transfection mixture was then added to the respective plates containing cells and incubated for $4 \mathrm{~h}$ at $37^{\circ} \mathrm{C}$. After 4 $\mathrm{h}$, the transfection medium was removed, the cells were washed with 1XPBS and fresh medium with antibiotics was added.

\subsubsection{Stable transfection with plasmid DNA}

To create a stable cell line MCF10A or MDA-MB-231 cells were transfected with respective linearized plasmid DNA (using Vspl) as described in section 3.1.2. After transfection, cells were grown for $4 \mathrm{~h}$ in media without antibiotics and then the media was replaced with media containing antibiotics. Then the cells were trypsinized, diluted and transferred to new plates. The cells were grown in a selective media containing Hygromycin $(100 \mu \mathrm{g} / \mathrm{ml})$. The cells were grown in a selection medium for 3-4 weeks and these cells were further used for the later experiments. 


\subsubsection{Migration assay}

To monitor the migration potential of the cells, a transwell migration assay was performed. The stable cell lines (MCF10A and MDA-MB-231 with stable ASXL1 ectopic expression) or cells (MCF10A and MCF12A) transfected with respective siRNAs were used for this experiment. To study migration of the cells, $8.0 \mu \mathrm{m}$ PET track-etched membrane cell culture inserts, pre-equilibrated with serum-free medium for at least 20-30 mins, were used. The cells were trypsinized and approximately 50,000 MCF10A and MCF12A cells and 20,000 MDA-MB-231 cells were seeded into the cell culture inserts with normal culture medium and cells were allowed to migrate through the membrane. The cells were cultured for further $48 \mathrm{~h}$ and the cells were gently scraped from the upper side of the membrane using a Q-Tip. 100\% methanol was used for the fixation of the migrated cells for 10 mins. Migrated cells were then stained using crystal violet staining (1\% (w/v) crystal violet dissolved in $20 \%(\mathrm{v} / \mathrm{v})$ ethanol) for 10 mins. The inserts were rinsed twice with distilled water to remove the excess staining and were allowed to dry. The crystal violet stained membrane with the migrated cells was visualized under the microscope and the images were taken using Nikon light microscope.

\subsubsection{Mammosphere formation assay}

In vitro proliferative capacity of the single-cell suspension was determined by mammosphere formation assay. In this method, single cells were grown in nondifferentiating and non-adherent conditions. The cell lines (stable cell lines or siRNA transfected) were trypsinized to form single-cell suspensions and were seeded at a density of nearly 2500 cells/ml in DMEM/F12 medium supplemented with $2 \%(\mathrm{v} / \mathrm{v})$ B27 (serum-free supplement), $10 \mu \mathrm{g} / \mathrm{ml}$ insulin, $0.5 \mu \mathrm{g} / \mathrm{ml}$ hydrocortisone, $20 \mathrm{ng} / \mathrm{ml}$ 
basic fibroblast growth factor (bFGF) and $20 \mathrm{ng} / \mathrm{ml}$ human epidermal growth factor (EGF) were seeded into Corning ${ }^{\circledR}$ flat bottom ultra-low-attachment 96 well microplates $(200 \mu \mathrm{l})$. The cells were fed every $48 \mathrm{~h}$ by addition of fresh medium and grown for 6 days. The mammospheres formed were counted and images were taken using Nikon light microscope. Images were analyzed (used Celigo) and quantified and represented as "relative number of spheres". The statistical significance of the triplicates was calculated.

\subsection{Molecular Biology}

\subsubsection{Molecular cloning}

The ASXL1 construct used in this study was obtained by amplification of synthetic ASXL1 and cloning into pSG5-HA-P2A-ERT2-Hygromycin vector.

\subsubsection{Polymerase chain reaction (Scott, Korfi et al.)}

The pCMV6 plasmid with human ASXL1 (NM_015338) with an N-terminal FLAG tag was a generous gift from Dr. Ross L. Levine (Memorial Sloan-Kettering Cancer Center (MSKCC)) (Abdel-Wahab, Adli et al. 2012). This plasmid was used as a template for the amplification of hASXL1. The PCR reaction was set up as follows: $10 \mu \mathrm{l}$ of $5 \mathrm{x}$ Phusion High-Fidelity buffer, $100 \mathrm{ng}$ of plasmid template, dNTPs mix to a final concentration of $200 \mu \mathrm{M}, 5 \mu \mathrm{M}$ of sense and $5 \mu \mathrm{M}$ of antisense primers, 1 unit of Phusion ${ }^{\circledR}$ High-Fidelity DNA Polymerase and the total volume was made up to $50 \mu \mathrm{l}$ by adding water.

A PCR reaction was performed using the following gradient protocol. 


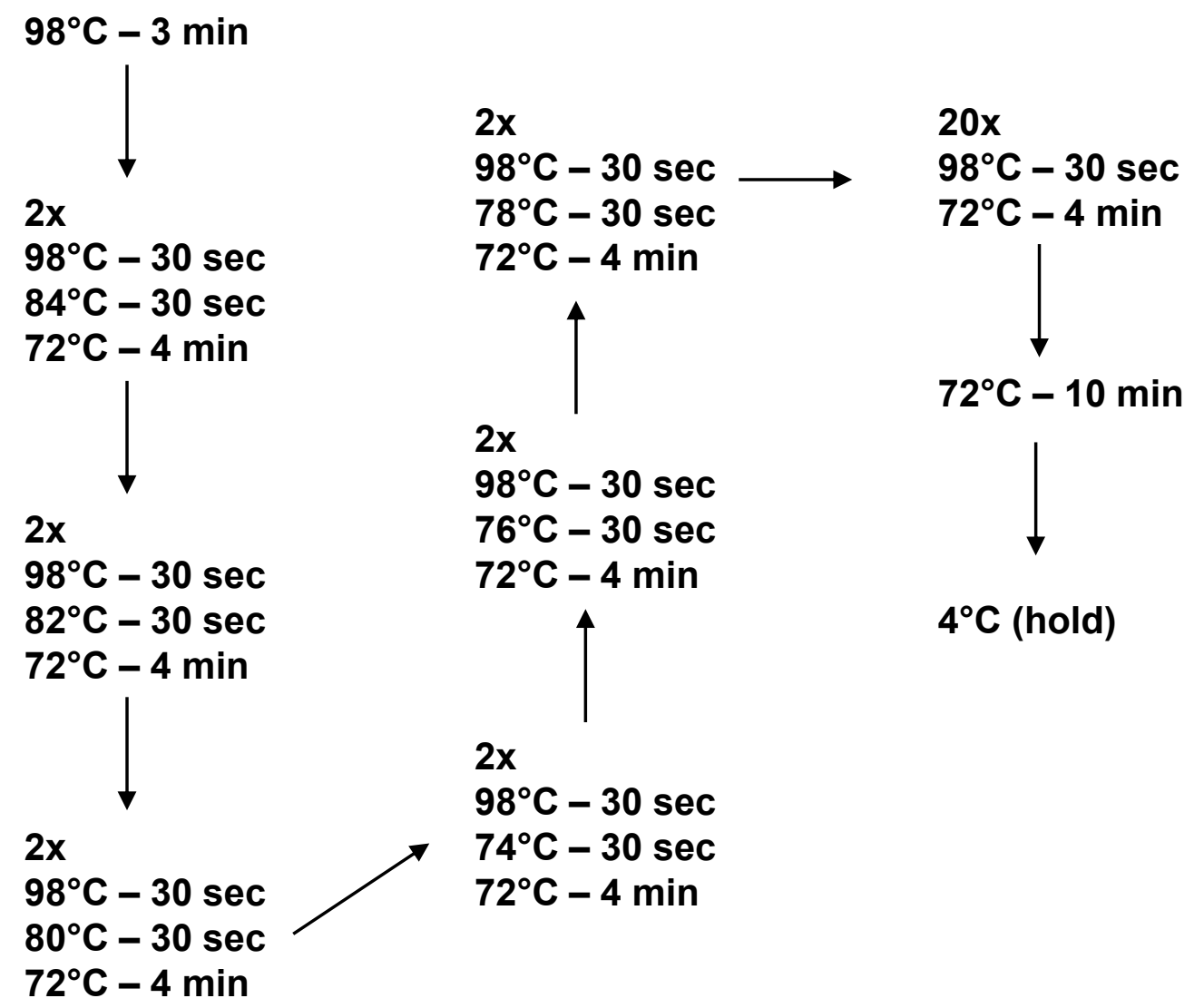

The final PCR product was analyzed on a $1 \%$ agarose gel with appropriate molecular weight markers and purified by using agarose gel purification kit (Bioline).

\subsubsection{Restriction enzyme digestion}

To clone hASXL1 into a pSG5 vector, restriction enzyme digestion was performed to cut the hASXL1 PCR product and the vector using Notl and Spel. The restriction digestion mixture was set up as follows: $2-4 \mu \mathrm{g}$ of DNA, $3 \mu \mathrm{l}$ of $10 \mathrm{x}$ restriction enzyme buffer, 5 units of each restriction enzyme, made up with $\mathrm{ddH}_{2} \mathrm{O}$ to a total volume of $30 \mu \mathrm{l}$. The reaction was incubated at $37^{\circ} \mathrm{C}$ for overnight followed by heat inactivation of restriction enzymes at $95^{\circ} \mathrm{C}$ for $10 \mathrm{~min}$. The digested PCR products or 
vectors were separated on a $1 \%$ agarose gel and subsequently gel eluted for further use.

\subsubsection{Agarose gel purification of DNA fragments}

DNA fragments of interest (amplified PCR products, digested PCR products and vector) were purified by electrophoresis on a $1 \%$ low melting agarose gel in $1 \mathrm{x}$ TAE. The samples were loaded along with molecular size markers to aid in excising out the right sized band of interest in a UV transilluminator. The DNA in the gel piece was isolated using agarose gel purification kit (Bioline).

\subsubsection{DNA ligation}

In order to ligate the hASXL1 insert into the pSG5-HA-ERT2-P2A-Hygromycin vector, the ligation mixture was set up as follows: $100 \mathrm{ng}$ of restriction enzyme digested vector DNA, 300 ng of restriction enzyme digested insert DNA (ratio vector: insert, approximately 1:3), $2 \mu \mathrm{l}$ of $10 \mathrm{x}$ ligase buffer, $400 \mathrm{U}$ of T4 DNA ligase and reaction volume was made up to $20 \mu \mathrm{l}$ with $\mathrm{ddH}_{2} \mathrm{O}$. The ligation reaction was performed at $16^{\circ} \mathrm{C}$ overnight.

\subsubsection{Heat shock transformation}

For transformation, the competent bacterial $(\mathrm{DH} 5 \alpha)$ cells were thawed on ice and incubated with DNA or ligation mixture on ice for 20-30 min with occasional mixing. After incubation, the cells were applied with heat shock at $42^{\circ} \mathrm{C}$ for $90 \mathrm{sec}$ followed by chilling on ice for $3-5 \mathrm{~min}$. The cells then were supplemented with $1 \mathrm{ml}$ of LB medium and incubated at $37^{\circ} \mathrm{C}$ for $1 \mathrm{~h}$ followed by centrifugation. The supernatant was discarded and the cell pellet was resuspended in $200 \mu \mathrm{l}$ of LB medium, which was then plated onto LB agar plates containing the appropriate antibiotic. The plates 
were incubated at $37^{\circ} \mathrm{C}$ overnight in a bacterial incubator after which the colonies were picked for further screening.

\subsubsection{Screening of recombinant clones and plasmid isolation}

The bacterial colonies obtained in the above protocol were randomly picked and inoculated into $3 \mathrm{ml}$ LB medium with ampicillin and grown to saturation. The culture was centrifuged at $12,000 \mathrm{rpm}$ for one $\min$ at $4^{\circ} \mathrm{C}$ and the supernatant was discarded. The cell pellet was resuspended, lysed and neutralized using MachereyNagel buffers. The contents were spun at $11,000 \mathrm{~g}$ for $10 \mathrm{~min}$ at $4^{\circ} \mathrm{C}$ and the supernatant containing the plasmid was transferred to a fresh microfuge tube. The nucleic acids were precipitated by adding an equal volume of isopropanol at room temperature for $20 \mathrm{~min}$ and stored at $-80^{\circ} \mathrm{C}$ for at least an hour. The DNA was pelleted by centrifuging at $11,000 \mathrm{~g}$ for 10 min.at $4^{\circ} \mathrm{C}$. The pellet was washed with $70 \%$ alcohol, dried and resuspended in a minimum volume of autoclaved water. An aliquot of the DNA was then digested with suitable restriction enzymes to release the insert analyzed $1-1.5 \%$ agarose gel electrophoresis. The positive clones were further confirmed by DNA sequencing. The positive bacteria culture was used to inoculate $100 \mathrm{ml}$ of LB medium supplemented with Ampicillin and incubated with shaking at $37^{\circ} \mathrm{C}$ overnight. On the next day, the cells were pelleted and the plasmid DNA was purified using the Pure Macherey-Nagel (MN) Plasmid Midiprep kit according to the manufacturer's instructions.

\subsubsection{RNA isolation}

RNA was isolated from the cultured cells using QIAzo|® reagent according to the manufacturer's instructions. Culture medium was aspirated and cells were washed twice with 1XPBS. The cells were lysed by adding $500 \mu$ l of QIAzol $尺$ reagent to each 
well (6 well plates), scraped and collected into $1.5 \mathrm{ml}$ tubes. $100 \mu \mathrm{l}$ of chloroform was added to the samples, the tubes were then vortexed approximately $20 \mathrm{sec}$ and centrifuged at $12,000 \mathrm{~g}$ for $20 \mathrm{~min}$ at $4^{\circ} \mathrm{C}$. The upper aqueous phase was collected into a fresh $1.5 \mathrm{ml}$ tube and then samples were precipitated with isopropanol at $80^{\circ} \mathrm{C}$ for $2 \mathrm{~h}$. After $2 \mathrm{~h}$ the samples were centrifuged at $12,000 \mathrm{~g}$ for 30 min at $4^{\circ} \mathrm{C}$. The pellets formed were washed with $70 \%$ ethanol, dried and resuspended in $40 \mu \mathrm{l}$ of DEPC water. RNA concentration was measured using a NanoDrop. The isolated RNA was used for cDNA synthesis or RNA-sequencing.

\subsection{3 cDNA synthesis}

For cDNA synthesis $1 \mu \mathrm{g}$ of total RNA was mixed with $6 \mu \mathrm{l}$ of a master mix containing $4 \mu \mathrm{l}$ of $2.5 \mathrm{mM}$ dNTP mix and $2 \mu \mathrm{l}$ of $15 \mu \mathrm{M}$ random primers. DEPC water was added to make the volume up to $16 \mu \mathrm{l}$. The mixture was then incubated at $70^{\circ} \mathrm{C}$ for $5 \mathrm{~min}$ followed by cooling on ice for few sec. To this, $4 \mu$ of reverse transcription master mix containing $2 \mu \mathrm{l}$ of $10 \mathrm{x}$ reaction buffer, 10 units of RNAse inhibitor, 25 units of reverse transcriptase M-MuLV and $1.625 \mu \mathrm{l}$ of DEPC water was added to each sample. cDNA synthesis was performed at $42^{\circ} \mathrm{C}$ for 1 hour. Then the enzyme was inactivated at $95^{\circ} \mathrm{C}$ for $5 \mathrm{~min}$. Finally, cDNA was diluted up to $50 \mu \mathrm{l}$ with DEPC water.

\subsubsection{Quantitative real-time PCR}

Quantitative real-time PCR was performed with a final reaction volume of $25 \mu \mathrm{l}$. One $\mu \mathrm{l}$ of cDNA or ChIP DNA and $24 \mu \mathrm{l}$ of master mix was used for each reaction. Each $24 \mu \mathrm{l}$ of master mix contains $14 \mu \mathrm{l}$ of the qRT-PCR mix, $1.5 \mu \mathrm{l}$ of $5 \mu \mathrm{M}$ primer mix (forward and reverse) and $8.5 \mu \mathrm{l}$ of $\mathrm{ddH}_{2} \mathrm{O}$. qRT-PCR mixture consists of $20 \mathrm{mM}$ $\left(\mathrm{NH}_{4}\right) \mathrm{SO}_{4}, 3 \mathrm{mM} \mathrm{MgCl}, 200 \mu \mathrm{M}$ dNTPs, $75 \mathrm{mM}$ Tris- $\mathrm{HCl}(\mathrm{pH}$ 8.8), 0.01\% Tween- 
20, 0.5 U/reaction Taq DNA Polymerase. 0.25\% Triton X-100, 1:80,000 SYBR Green I and $300 \mathrm{mM}$ Trehalose.

A two-step PCR reaction was performed using the following protocol.

\section{$95^{\circ} \mathrm{C}-2 \mathrm{~min}$}

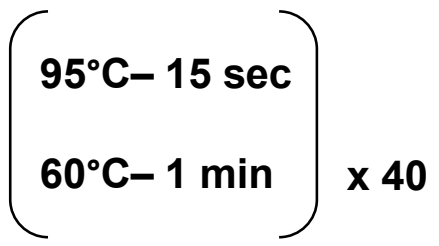

The PCR reaction was followed by a melting curve analysis from $60^{\circ} \mathrm{C}$ to $95^{\circ} \mathrm{C}$ with read every $0.5^{\circ} \mathrm{C}$.

cDNA was quantified using a standard curve made from all cDNA samples. HNRNPK or 36B4 primers were used as an internal reference gene to normalize all qRT-PCR samples before the statistical analysis. The expression levels were represented as relative to the control samples and expressed as "relative mRNA expression".

A standard curve made from the ChIP input DNA was used for the quantification of ChIP and ChIP input samples. ChIP samples were normalized to their corresponding input samples and represented as "\% of input".

\subsubsection{Chromatin immunoprecipitation (ChIP)}

\subsubsection{Cross-linking and sonication}

For ChIP experiments, cells were grown in either $10 \mathrm{~cm}$ or $15 \mathrm{~cm}$ plates. ProteinDNA complexes in cells were cross-linked with $1 \%$ formaldehyde in 1 XPBS for 20 min (ASXL1 ChIP) or 10 min (histone modifications ChIP) at RT. 1.25 M glycine was 
added in order to quench the formaldehyde and incubated for $5 \mathrm{~min}$. Cells were washed twice with ice- cold 1 XPBS and $1 \mathrm{ml}$ of Nelson buffer containing protease inhibitor cocktail was added. Then the cells were scraped and collected into $1.5 \mathrm{ml}$ tubes. The nuclear pellet was isolated by centrifugation at $12,000 \mathrm{~g}$ for $1 \mathrm{~min}$ at $4^{\circ} \mathrm{C}$ and the supernatant was discarded. The nuclear pellet was washed with $1 \mathrm{ml}$ of Nelson buffer and again centrifuged. The final nuclear pellet was resuspended in 200-250 $\mu \mathrm{l}$ of Gomes Lysis buffer and incubated on the rotor for $15 \mathrm{~min}$ at $4^{\circ} \mathrm{C}$. Samples were then sonicated using Bioruptor ${ }^{\circledR}$ Pico (Diagenode) at high power with $30 \mathrm{sec}$ on/off pulse for $15-30$ cycles. Sonicated samples were centrifuged at 12,000 $g$ at $8^{\circ} \mathrm{C}$ for $10 \mathrm{~min}$.

\subsubsection{Shearing check}

To check the sonication efficiency of chromatin, the shearing check was performed before proceeding with a pre-clearing step. $10 \mu \mathrm{l}$ of the sonicated chromatin sample was taken into a separate tube and $100 \mu$ l of Weinmann Lysis buffer (WB) and $1 \mu \mathrm{l}$ of Proteinase- $\mathrm{K}(20 \mathrm{mg} / \mathrm{ml})$ were added and incubated in a thermo-shaker at $65^{\circ} \mathrm{C}$ (800 rpm) overnight. Samples were then spun down and DNA was isolated by phenol/chloroform/isoamyl alcohol extraction as described below (for visualization pink precipitant was used). The isolated DNA pellet was dissolved in $15 \mu$ of $10 \mathrm{mM}$ Tris- $\mathrm{HCl}\left(\mathrm{pH}\right.$ 8) containing $100 \mu \mathrm{g} / \mathrm{ml}$ RNAse A and incubated for $1 \mathrm{~h}$ at $37^{\circ} \mathrm{C}(800$ rpm). Then the DNA was mixed with the loading dye and allowed to run on $1.5 \%$ agarose gel at $100 \mathrm{~V}$ followed by analysis on gel documentation. The shearing of DNA was considered efficient if the DNA fragments were concentrated around 150300 bp. 


\subsubsection{Pre-clearing and immunoprecipitation}

For pre-clearing, $100 \mu \mathrm{l}$ of $50 \%$ sepharose beads slurry was added to the chromatin extract and incubated on a rotor for $1 \mathrm{~h}$ at $4^{\circ} \mathrm{C}$. The samples were centrifuged and the supernatant was transferred to a fresh tube. The samples were diluted using Gomes Lysis Buffer containing proteinase inhibitor cocktail. Chromatin samples were aliquoted and samples were either snap frozen in liquid nitrogen and stored in $-80^{\circ} \mathrm{C}$ or proceeded for immunoprecipitation reaction. $10 \%$ of ChIP extract per corresponding ChIP sample was collected as input into a separate tube and snap frozen. For each immunoprecipitation reaction, $100 \mu \mathrm{l}$ of chromatin extract was diluted to $500 \mu \mathrm{l}$ with Gomes Lysis Buffer containing proteinase inhibitor cocktail and 1-2 $\mu \mathrm{g}$ of respective antibody was added and incubated overnight on a rotor at $4^{\circ} \mathrm{C}$. Chromatin complexes were pulled down by adding $30 \mu \mathrm{l}$ of $50 \%$ Protein-A sepharose slurry and incubated for $2 \mathrm{~h}$ at $4^{\circ} \mathrm{C}$. After $2 \mathrm{~h}$, samples were centrifuged at $2000 \mathrm{~g}$ for 2 min at $4^{\circ} \mathrm{C}$. The samples were washed with different ice-cold buffers as follows: twice with Gomes Lysis Buffer, twice with Gomes Wash Buffer, twice with Gomes Lysis Buffer and twice with TE buffer. The samples were mixed gently with the buffers during washings.

\subsubsection{DNA isolation}

Immunoprecipitated chromatin complexes and input samples were treated with $15 \mu \mathrm{l}$ of RNAse A $(10 \mu \mathrm{g})$ diluted in $10 \mathrm{mM}$ Tris- $\mathrm{HCl} \mathrm{pH} 8(0.2 \mu \mathrm{g} / \mu \mathrm{l})$ and incubated at $37^{\circ} \mathrm{C}$ for $30 \mathrm{~min}$. Protein bound to DNA was removed by treating the samples with 1 $\mu \mathrm{l}$ of Proteinase- $\mathrm{K}(20 \mathrm{mg} / \mathrm{ml})$ and incubated at $65^{\circ} \mathrm{C}$ on a thermo-shaker overnight. The samples were centrifuged at $2,000 \mathrm{~g}$ for $2 \mathrm{~min}$ at RT and supernatant was transferred to a separate tube. DNA was precipitated by adding $10 \mu \mathrm{l}$ of $8 \mathrm{M} \mathrm{LiCl}$ and 
$4 \mu \mathrm{l}$ of colorless co-precipitant (Bioline). Subsequently, 200 $\mu \mathrm{l}$ of phenol/chloroform/isoamyl alcohol (25:24:1) was added, vortexed for $30 \mathrm{sec}$ and centrifuged at a maximum speed at $37^{\circ} \mathrm{C}$ for $2 \mathrm{~min}$. The aqueous phase was transferred to a fresh tube and back extraction was performed by adding $200 \mu \mathrm{l}$ of 10 $\mathrm{mM}$ Tris- $\mathrm{HCl} \mathrm{pH} 8+0.4 \mathrm{M} \mathrm{LiCl}$ and vortexed for $30 \mathrm{sec}$. Samples were centrifuged at a full speed and the aqueous phase was collected and pooled with the first one. Precipitation was performed by adding $1 \mathrm{ml}$ of $100 \%$ ethanol for $2 \mathrm{~h}$ at $-80^{\circ} \mathrm{C}$ followed by centrifugation at $15,000 \mathrm{~g}$ for $30 \mathrm{~min}$ at $4^{\circ} \mathrm{C}$. DNA pellets were washed with $70 \%$ ethanol and centrifuged at $15,000 \mathrm{~g}$ for $5 \mathrm{~min}$. The pellets were dried and resuspended in $40 \mu \mathrm{l}$ of water. $5 \mu \mathrm{l}$ of ChIP DNA was aliquoted into a fresh tube and diluted for analysis by quantitative real-time PCR to check the efficiency of ChIP. The background (nonspecific) binding was determined by performing a ChIP with a nonspecific IgG antibody. ChIP samples were normalized to input DNA samples, and displayed as "\% of input".

\subsection{Protein biochemistry}

\subsubsection{Whole cell extract preparation}

For total protein extraction, the cells were washed with 1XPBS and lysed in ice-cold RIPA buffer containing proteinase inhibitor cocktail (1 mM Pefabloc, 10 mM BGP, 1 $\mathrm{mM}$ NEM and $1 \mathrm{ng} / \mu \mathrm{l}$ Aprotinin/Leupeptin). The extracts were then sonicated for 15 cycles for $30 \mathrm{sec}$ on/off pulse using a Bioruptor® Pico (Diagenode) at high power to shear genomic DNA. 


\subsubsection{SDS polyacrylamide gel electrophoresis}

After sonication, the protein samples were boiled with Laemmli Buffer for $10 \mathrm{~min}$ at $95^{\circ} \mathrm{C}$. The proteins were separated on sodium dodecyl sulfate (SDS) polyacrylamide gels (SDS-PAGE) (Laemmli 1970). The resolving and stacking gel were prepared as described in the Materials section. Polyacrylamide gels were run in SDS running buffer at $100 \mathrm{~V}$ till the dye front reached the bottom of the gel.

\subsubsection{Immunoblot analysis and detection}

The proteins resolved according to their molecular weight by SDS-PAGE were later detected by immunoblot (Towbin, Staehelin et al. 1992) using specific antibodies against the protein of interest. Separated proteins were then transferred at $100 \mathrm{~V}$ to nitrocellulose membrane for $90 \mathrm{~min}$. The membrane with transferred proteins was then incubated with blocking solution (5\% skimmed milk in TBST) at room temperature for $1 \mathrm{~h}$ to block non-specific antibody binding. Then the membrane was incubated at $4^{\circ} \mathrm{C}$ for overnight with appropriate primary antibody diluted in blocking solution as described in the materials section. On the next day, membrane was washed thrice with TBST buffer and incubated for $1 \mathrm{~h}$ with horseradish peroxidaseconjugated secondary antibody diluted in blocking solution at RT. Membrane was then washed thrice with TBST buffer and analyzed for HRP signals by using Enhanced chemiluminescence reagent and further exposed in western blot imager (Biorad) or on X-ray films

\subsubsection{Co-immunoprecipitation}

Cells were washed with 1XPBS and scraped in $1.0 \mathrm{ml}$ of ice-cold Co-IP lysis buffer containing protease inhibitors and incubated for $1 \mathrm{~h}$ on a rotator at $4^{\circ} \mathrm{C}$. The cells 
were sonicated and centrifuged at $10,000 \mathrm{rpm}$ for $10 \mathrm{~min}$ at $4^{\circ} \mathrm{C}$. The supernatant was then transferred to a fresh $1.5 \mathrm{ml}$ tube and resonicated followed by centrifugation. The supernatant was transferred to a fresh tube and $100 \mu \mathrm{l}$ was collected to use as input. An appropriate amount of supernatant was incubated with 1-2 $\mu \mathrm{g}$ of antibody for overnight at $4^{\circ} \mathrm{C}$ on a rotator. The next day, $30 \mu \mathrm{l}$ of $50 \%$ slurry of Protein $A$ or $\mathrm{G}$ sepharose was added and incubated for $2 \mathrm{~h}$ on a rotator at $4^{\circ} \mathrm{C}$. Beads were collected by centrifugation at $1000 \mathrm{rpm}$ for $2 \mathrm{~min}$, washed twice with lysis buffer and resuspended in $50-100 \mu \mathrm{l}$ of lysis buffer. Beads were boiled in 6x Laemmli buffer, loaded on SDS-PAGE and proteins of interest were detected using specific antibodies.

\subsubsection{Immunofluorescence}

MCF10A cells were transfected with respective siRNAs and grown on chamber slides. The cells were washed with 1XPBS and then fixed with $4 \%$ paraformaldehyde (PFA) for $20 \mathrm{~min}$ at RT. Cells were washed thrice with $1 \mathrm{x}$ PBS to remove the residual PFA and permeabilized using $0.1 \%$ Triton $\mathrm{X}-100$ for $10 \mathrm{~min}$. After washing thrice with1XPBS, cells were blocked with 3\% BSA (bovine serum albumin) for 20 min followed by overnight incubation with primary antibody diluted in 3\% BSA. Next day, unbound antibody was washed away thrice with 1XPBS and incubated with Alexa-488 or Alexa-594 conjugated secondary antibodies diluted in 3\% BSA. Cells were washed thrice with 1 XPBS and the nuclei were stained with DAPI followed by 1xPBS wash three times. The samples were mounted with coverslips using the mounting medium. Images were taken using Leica inverted-2 confocal microscope followed by processing of images using the LSM Image browser. 


\subsection{Next generation sequencing}

\subsubsection{RNA sequencing}

RNA sequencing was performed in duplicates. RNA integrity was checked by visualizing the RNA bands on a $1 \%$ formaldehyde-agarose gel before proceeding for library preparation. The RNA samples with proper integrity were used for library preparation.

\subsubsection{Library preparation}

RNA library preparation was performed using the NEBNext ${ }^{\circledR}$ Ultra ${ }^{T M}$ Library Prep according to the manufacturer's instructions. $1 \mu \mathrm{g}$ of total RNA was used for the downstream processing. From the total RNA, mRNA enrichment was performed using polyadenylated magnetic beads. The enriched mRNA was fragmented by incubating the samples at $94^{\circ} \mathrm{C}$ for $15 \mathrm{~min}$ in a thermocycler. Fragmented and primed mRNA was used for the first and the second strand cDNA synthesis. Doublestranded cDNA was purified using Agencourt AMPure XP beads and afterward end repair reaction (single base overhangs at $5^{\prime}$ end) which was then followed by adaptor ligation (sequencing adaptor) and purification steps. Purified adaptor-ligated cDNA samples were PCR amplified by using universal primer for all samples and a specific index primer for each sample which later allows the separation of individual sample loaded on the same lane of the sequencer.

\subsubsection{Chromatin immunoprecipitation sequencing (ChIP-seq)}

After confirming the efficiency of chromatin immunoprecipitation by quantitative realtime PCR, isolated ChIP DNA was used for sequencing. The concentration of isolated ChIP DNA was measured using a Qubit dsDNA HS assay on a Qubit ${ }^{2} 2.0$ 
Fluorometer before starting the library preparation. ChIP sequencing was performed in duplicates.

\subsubsection{Library preparation}

2-10 ng of ChIP DNA was used for the library preparation. DNA samples were resonicated to make fragments of $200-300$ bp size using Bioruptor® Pico (Diagenode). Then fragmented DNA was used for the library preparation. Library preparation was performed using NEBNext Ultra DNA library preparation kit (New England Biolabs) for histone modifications and Microplex ${ }^{\mathrm{TM}}$ Library preparation kit v2 for ASXL1 from Diagenode $®$ according to the manufacturer's instructions. End prep reaction was set by adding end prep reaction buffer and end prep enzyme mix (total reaction volume $65 \mu \mathrm{l}$ ) to each sample and incubated at $20^{\circ} \mathrm{C}$ for $30 \mathrm{~min}$ and then at $65^{\circ} \mathrm{C}$ for $30 \mathrm{~min}$. Adaptor ligation reaction was set up by adding following reagents to the samples: Blunt/TA ligase master mix $(15 \mu \mathrm{l})$, ligation enhancer $(1 \mu \mathrm{l})$, NEBNext adaptor for Illumina $(2.5 \mu \mathrm{l})$ making the reaction volume to $83.5 \mu \mathrm{l}$. Then the samples were incubated at $20^{\circ} \mathrm{C}$ in a thermocycler for $15 \mathrm{~min} .3 \mu \mathrm{l}$ of USER enzyme was added to the samples and incubated at $37^{\circ} \mathrm{C}$ for $15 \mathrm{~min} .0 .9 \mathrm{x}$ AMPure $\mathrm{XP}$ magnetic beads were added to the adaptor-ligated DNA for size selection followed by two washes using $80 \%$ ethanol. Beads were dried and resuspended in $28 \mu \mathrm{l}$ of $10 \mathrm{mM}$ Tris- $\mathrm{HCl}$ pH 8.0. $23 \mu \mathrm{l}$ of purified adaptor ligated DNA was PCR amplified by using specific index primers (barcode) for each library, which later allows the separation of individual sample loaded on the same lane of the sequencer. PCR amplified DNA was cleaned using AMPure XP beads and the DNA was resuspended in $33 \mu \mathrm{l}$ of 10 $\mathrm{mM}$ Tris- $\mathrm{HCl} \mathrm{pH}$ 8.0. 
Each ChIP library DNA concentration was measured using Qubit ${ }^{\circledR}$ 2.0 Fluorometer and the fragment size of the libraries were analyzed using Agilent Bioanalyzer 2100 (High Sensitivity DNA assay). ChIP DNA libraries were pooled together to a final concentration of $10 \mathrm{nM}$ and then diluted to $2 \mathrm{nM}$. The pooled libraries were sequenced using HiSeq 2500 (Illumina) sequencer at the Transcriptome Analysis Laboratory (TAL), Göttingen.

\subsection{Bioinformatic analysis of RNA and ChIP sequencing data}

\subsubsection{RNA-sequencing data analysis}

RNA sequencing raw data was obtained as Fastq files and the quality was checked using the FastQC tool on Galaxy. Then the Fastq files were then mapped to the human genome (hg 19) using the TopHat tool (settings were set to 'very sensitive) (Kim, Pertea et al. 2013). SortSam (version 1.126.0) was used to sort the coordinates from Picard tools on Galaxy. The resulted files were then used for read counting using the HTSeq tool (version 0.6.0) (Anders, Pyl et al. 2015). The output htseqcount files were used for DEseq package in R-script (Bioconductor version 3.2) (Love, Huber et al. 2014). The list of differentially regulated genes was used for Gene Set Enrichment Analysis (GSEA). GSEA was performed with standard parameters (1000 permutations of gene sets, Signal2Noise ranking metric) and significantly enriched pathways (c5.all gene sets) were selected (Subramanian, Tamayo et al. 2005). Gene Ontology (GO) analysis was performed using the DAVID (Database for Annotation, Visualization, and Integrated Discovery) software. DAVID is a web-based program for analyzing the list of genes associated with the biological annotation. The significantly enriched biological pathways were selected. 


\subsubsection{ChIP-sequencing data analysis}

Raw ChIP sequencing data was obtained as Fastq files. The quality of the raw data was checked by running Fastq quality check (FastQC) tool on Galaxy (S.Andrews Babraham Institute). After checking the quality of the raw data, the data was further analyzed using publicly available servers (Galaxy, Galaxy/Cistome and Galaxy/deepTools).

\subsubsection{Mapping and Peak calling}

The Fastq files were mapped to the human genome (hg 19, GRch37) ((Langmead and Salzberg 2012) using Bowtie2 tool on Galaxy, which involves alignment of short

DNA reads to the human genome. The end result of the Bowtie2 was in SAM (Sequence Alignment Map) format which was then converted to BAM (Binary Alignment Map) format using SAM-to-BAM conversion tool ( $\mathrm{Li}$, Handsaker et al. 2009). BAM files were then used for Model- Based Analysis of ChIP-seq 2 (MACS2) tool (Zhang, Liu et al. 2008), which helps in the identification of the peaks for the protein binding, commonly referred as peak calling with p-values $\leq 10 \mathrm{e}-5$ and minimum FDR (q-value) cutoff for peak detection was set to 0.05 .

\subsubsection{Normalization and visualization of ChIP-sequencing data}

The generated Bed file containing the peak location and Wiggle (Wig) file containing signal profile was further used to analyze the genome-wide enrichment of proteins or histone modifications. The total number of mapped reads were then normalized using the reads per kilobase per million (RPKM) option. Bigwig file was used to visualize the enrichment at the individual genomic regions using Integrative Genomics Viewer (IGV) software (Robinson, Thorvaldsdottir et al. 2011). Cis- 
regulatory Element Annotation System (CEAS) was used to determine the enrichment of the ChIP regions at specific genomic locations compared to the whole genome (Shin, Liu et al. 2009). Bigwig files were used to generate aggregate profile plots over defined genomic regions (deep Tools and Cistrome). 


\section{Results}

In this study, the role of Additional Sex Combs Like 1 (ASXL1) in Epithelial-tomesenchymal transition (EMT) was examined. Our main goal was to reveal the potential role of ASXL1 as a tumor suppressor and understand the molecular mechanism involved in suppressing the stem cell phenotype in mammary epithelial and breast cancer cells. Cellular events such as cell migration, adhesion, invasion and stemness are essential for normal cells. Previous studies reported that ASXL1 is perturbed in different cancers but the mechanistic role of ASXL1 as a tumor suppressor and EMT suppression is not yet studied. We also focused on studying the epigenetic regulation of ASXL1 in EMT which is a potent driver for cancer progression and metastasis. For this we used cell lines derived from human breast tissue such as MCF10A, MCF12A and MDA-MB-231. MCF10A and MCF12A are normal mammary epithelial cells and MDA-MB-231 is breast cancer cells and they all were used as a model system to study EMT.

\subsection{Role of ASXL1 in breast cancer}

\subsubsection{Expression of ASXL1 is downregulated in breast cancer}

In order to demonstrate the functional role of ASXL1, we compared the expression of ASXL1 mRNA between normal and cancer tissues. For this purpose, we used publicly available 'Oncomine' database. The Oncomine database contains a huge collection of gene expression datasets for a wide range of cancers, which allows the users to examine the expression of individual genes in context to different cancers and also in comparison with normal tissues. Breast carcinoma datasets were utilized to check the expression of ASXL1 mRNA levels and found that ASXL1 mRNA levels 
were significantly downregulated in breast carcinoma compared to the normal breast tissues in four independent datasets (Fig. 6). Reduced levels of ASXL1 expression in breast cancer samples further supports the tumor suppressor role of ASXL1 in breast cancer.
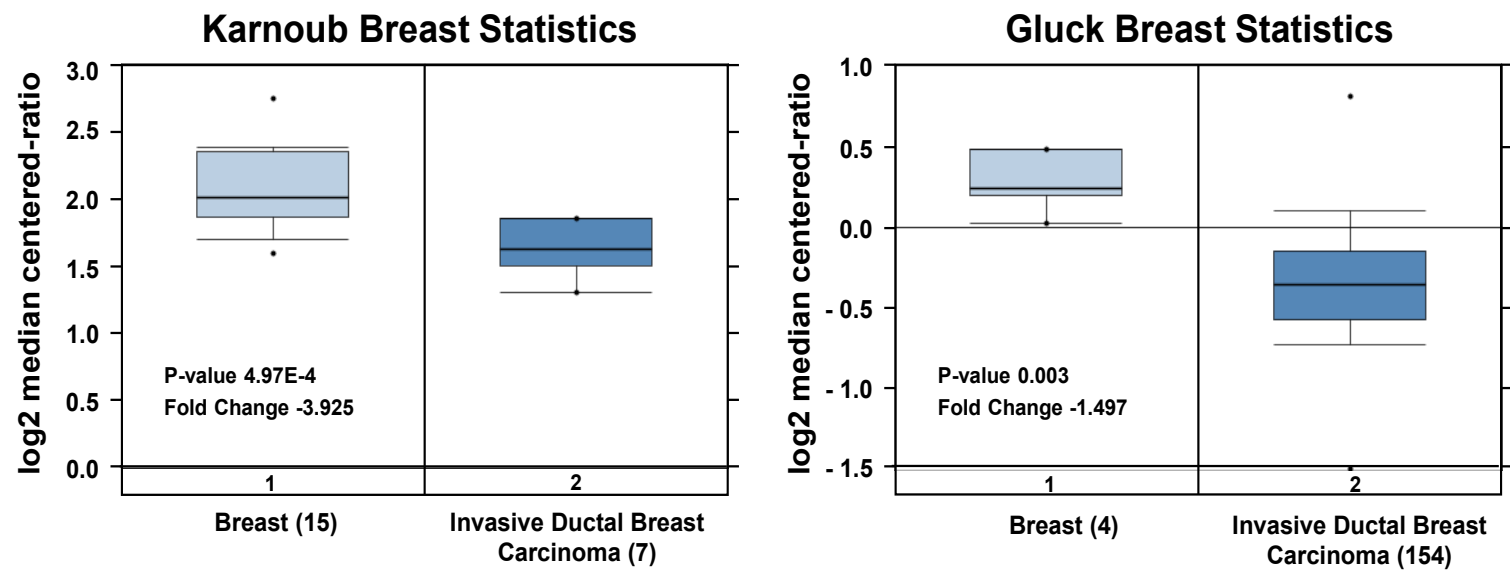

Finak Breast Statistics
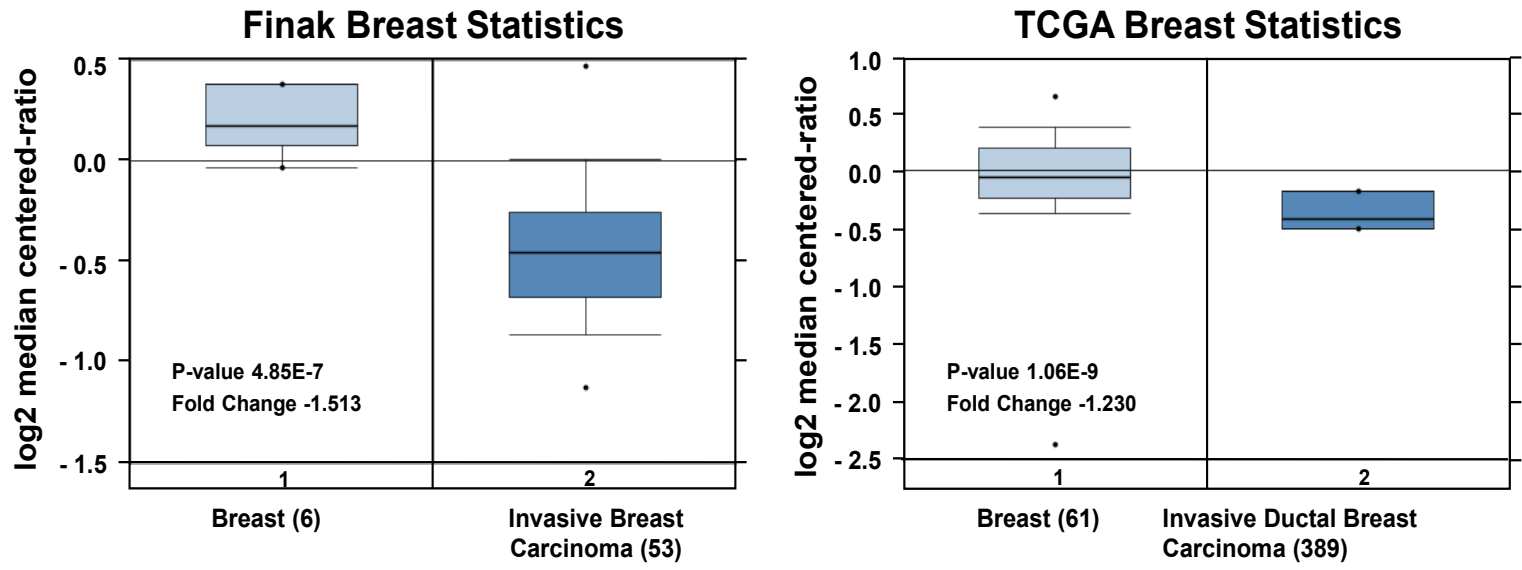

Figure. 6: ASXL1 gene expression in breast carcinoma. Expression of ASXL1 in breast cancer samples was found to be significantly downregulated when compared to the normal breast samples as depicted in four independent datasets. Publicly available Oncomine database was used to generate the above data.

\subsubsection{ASXL1 expression and survival analysis}

To investigate whether expression of ASXL1 can be used as a prognostic marker for the disease outcome in cancer patients, an online survival analysis tool, Kaplan- 
Meier Plotter was used to elucidate the prognostic role of ASXL1 in breast cancer. The Kaplan-Meier plotter is a publicly available online software, which can be used to assess the effect of genes on survival using several cancer samples. KaplanMeier relapse-free survival for all breast cancer patients was analyzed and we observed that breast cancer patients with low ASXL1 expression levels have poor relapse-free survival (RFS) compared to patients with high ASXL1 (Fig. 7).

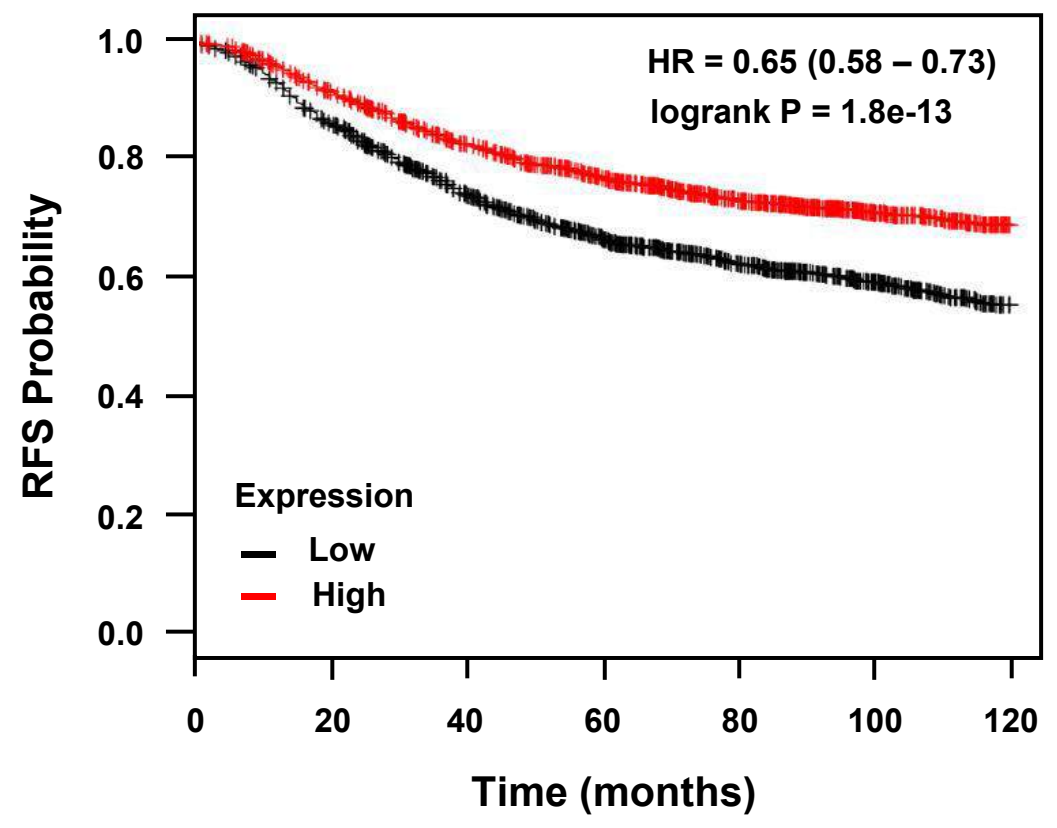

Figure. 7: ASXL1 expression level predicts the disease outcome. Kaplan - Meier plot for breast cancer showing that patients with low ASXL1 expression have significantly poor relapse-free survival rate compared to the patients with high ASXL1 expression.

\subsection{ASXL1 and epithelial-to-mesenchymal transition}

\subsubsection{ASXL1 downregulation perturbs the expression of EMT pathway genes}

As a first step towards understanding the biological functions of ASXL1 in mammary epithelial cells, we performed siRNA-mediated downregulation of ASXL1 in MCF10A cells (non-transformed mammary epithelial cells). As ASXL1 expression was perturbed in breast cancer patients, we employed MCF10A cell line. To perform 
transcriptome-wide analysis, MCF10A cells were transfected with either nontargeting control siRNA or ASXL1 siRNA. After $72 \mathrm{~h}$, total RNA was isolated and was used for mRNA sequencing. A thorough analysis of differentially regulated genes was carried out, where the genes are initially sorted based on fold change and significance values. For further analyses the genes that are differentially perturbed, up regulated $(F C \geq 1.0)$ or down regulated $(F C \leq 0.65)$ with a padj $\leq 0.05$ were selected.

As assumed, a significant fraction of EMT regulated genes was affected by depletion of ASXL1 compared with control (ASXL1 vs Control). However, some genes are not affected by depletion of $A S X L 1$, which implies that $A S X L 1$ is not required for their regulation. Interestingly, a number of other genes were differentially regulated by ASXL1 loss. To understand the mechanistic role of the ASXL1-regulated genes, we employed DAVID (Database for Annotation, Visualization, and Integrated Discovery), a web-based Gene ontology application. DAVID was used to determine the GO terms related to transcriptome-wide regulation and several biological pathways regulating important functions such as cell adhesion, motility were identified upon ASXL1 depletion (Fig. 8A). One of the primary and crucial characteristics of epithelial and differentiated cells in the tissues is to adhere to the extracellular matrix and to the adjacent cells. However, during metastasis, the altered cells eventually lose cell to cell and also cell to extracellular matrix contacts, enabling them to migrate to distant sites.

To further understand the role of ASXL1-regulated genes, we employed GSEA (Gene Set Enrichment Analysis), which identifies the significantly enriched pathways, which are proposed for specific sets of genes. Using 'c2.all' curated gene set for 
GSEA analysis, we could identify significantly enriched pathways associated with EMT and epithelial differentiation pathways in control conditions compared to ASXL1 depletion (Fig. 8B). GSEA analysis suggests that the ASXL1 loss results in an EMTlike phenotype, which may lead to metastasis. Furthermore, it also revealed an enrichment of gene signatures associated with a mammary stem cell phenotype and EMT pathways upon ASXL1 depletion. The graphical representation (enrichment plots) of pathways for epithelial differentiation and EMT identified from GSEA were shown in Fig. 8C.

A

\begin{tabular}{|c|c|c|c|c|c|}
\hline GO_TERM & $\begin{array}{l}\text { Enriched } \\
\text { Pathways }\end{array}$ & Count & $\%$ List & P-Value & $\begin{array}{c}\text { Benjamini } \\
\text { (FDR) }\end{array}$ \\
\hline SP_PIR_KEYWORDS & Proto-oncogene & 20 & 5.3 & 1.10E-07 & 2.60E-05 \\
\hline GOTERM_BP_FAT & $\begin{array}{l}\text { Response to } \\
\text { wounding }\end{array}$ & 34 & 9 & $9.20 \mathrm{E}-07$ & 2.20E-03 \\
\hline GOTERM_BP_FAT & $\begin{array}{c}\text { Regulation of cell } \\
\text { proliferation }\end{array}$ & 41 & 10.8 & $1.00 \mathrm{E}-05$ & 4.10E-03 \\
\hline GOTERM_BP_FAT & $\begin{array}{c}\text { Regulation of cell } \\
\text { death }\end{array}$ & 42 & 11.1 & $9.90 \mathrm{E}-06$ & $4.80 \mathrm{E}-03$ \\
\hline SP_PIR_KEYWORDS & Cell adhesion & 20 & 5.3 & 5.70E-04 & $1.70 \mathrm{E}-02$ \\
\hline GOTERM_BP_FAT & $\begin{array}{l}\text { Biological } \\
\text { adhesion }\end{array}$ & 33 & 8.7 & $5.60 \mathrm{E}-04$ & 4.90E-02 \\
\hline GOTERM_BP_FAT & $\begin{array}{l}\text { regulation of cell- } \\
\text { cell adhesion }\end{array}$ & 5 & 1.3 & $1.90 \mathrm{E}-03$ & $9.00 \mathrm{E}-02$ \\
\hline GOTERM_BP_FAT & $\begin{array}{c}\text { Locomotary } \\
\text { behaviour }\end{array}$ & 16 & 4.2 & $3.30 \mathrm{E}-03$ & $1.20 \mathrm{E}-01$ \\
\hline GOTERM_BP_FAT & Cell motion & 23 & 6.1 & $3.40 \mathrm{E}-03$ & $1.20 \mathrm{E}-01$ \\
\hline GOTERM_BP_FAT & Angiogenesis & 11 & 2.9 & $3.60 \mathrm{E}-03$ & $1.20 \mathrm{E}-01$ \\
\hline GOTERM_BP_FAT & $\begin{array}{l}\text { Blood vessel } \\
\text { development }\end{array}$ & 15 & 4 & $3.00 \mathrm{E}-03$ & 1.20E-01 \\
\hline GOTERM_BP_FAT & $\begin{array}{c}\text { Response to } \\
\text { hypoxia }\end{array}$ & 10 & 2.6 & $5.90 \mathrm{E}-03$ & $1.60 \mathrm{E}-01$ \\
\hline
\end{tabular}


B

\begin{tabular}{|c|c|c|}
\hline Gene Set Name & Size & NES \\
\hline BOSCO_EPITHELIAL_DIFFERENTIATION_MODULE & 63 & 2.03 \\
\hline AMIT_SERUM_RESPONSE_120_MCF10A & 61 & 1.94 \\
\hline CHARAFE_BREAST_CANCER_LUMINAL_VS_MESENCHYMAL_UP & 415 & 1.90 \\
\hline CHARAFE_BREAST_CANCER_BASAL_VS_MESENCHYMAL_UP & 114 & 1.84 \\
\hline JAEGER_METASTASIS_DN & 246 & 1.84 \\
\hline KOBAYASHI_EGFR_SIGNALING_24HR_UP & 92 & 1.84 \\
\hline SARRIO_EPITHELIAL_MESENCHYMAL_TRANSITION_DN & 92 & 1.82 \\
\hline WANG_HCP_PROSTATE_CANCER & 106 & 1.62 \\
\hline LIM_MAMMARY_STEM_CELL_DN & 395 & 1.58 \\
\hline ONDER_CDH1_TARGETS_2_DN & 438 & 1.43 \\
\hline
\end{tabular}


C

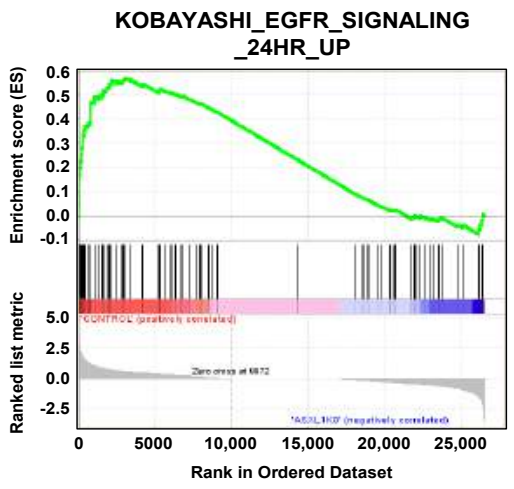

CHARAFE BREAST CANCER LUMINAL _VS_MESENCHYMAL_UP
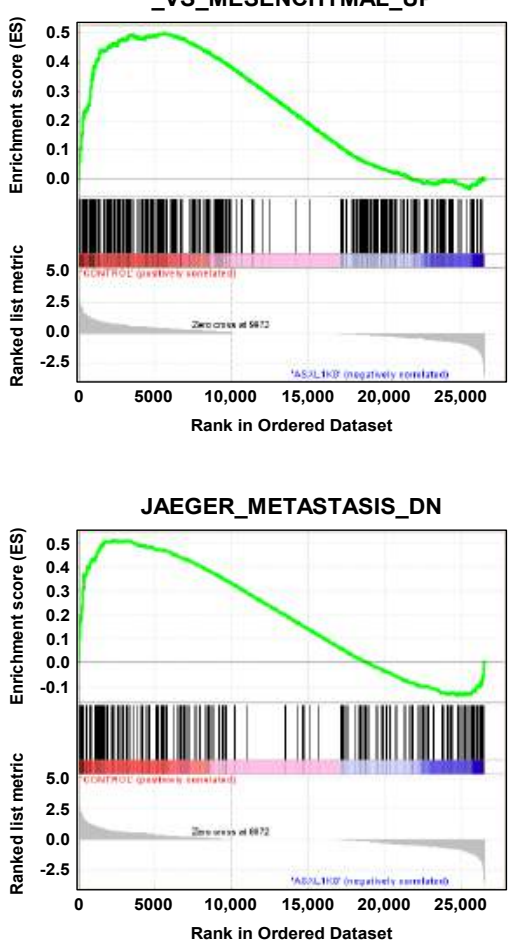

SARRIO_EPITHELIAL_MESENCHYMAL TRANSITION_DN

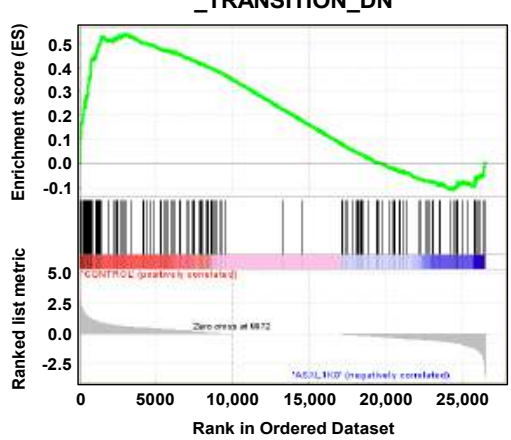

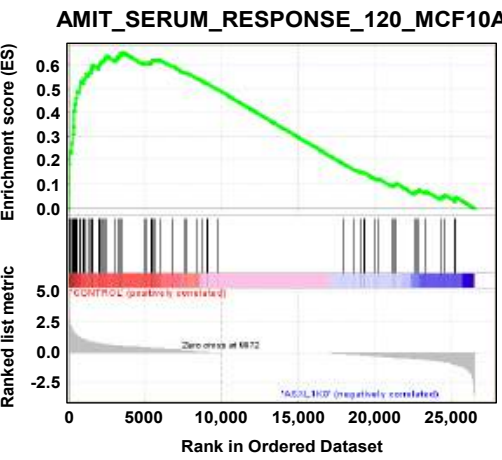

BOSCO_EPITHELIAL_DIFFE-

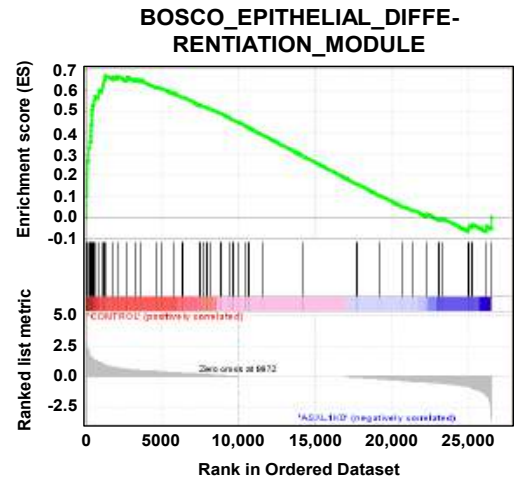

CHARAFE_BREAST_CANCER_BASAL

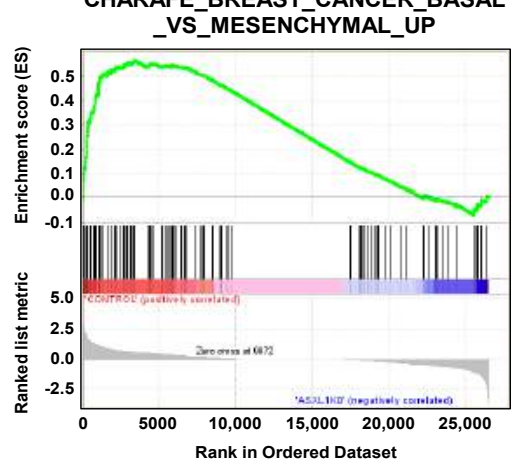

WANG_HCP PROSTATE CANCER

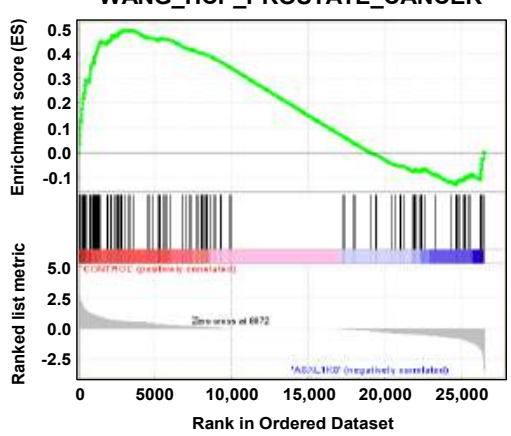

Figure. 8: ASXL1 regulate EMT and metastasis properties. (A) The upregulated and downregulated genes were used for DAVID Gene Ontology analysis. The significantly enriched biological pathways involved in cell migration and adhesion. (B and C) GSEA analysis recognizes EMT and epithelial differentiation pathways. 


\subsubsection{Loss of ASXL1 enhances EMT phenotype}

To elucidate the effect of ASXL1 expression on EMT phenotype, we performed several cell culture based assays in MCF10A and MCF12A cells. During EMT, epithelial cells lose their epithelial markers like E-cadherin and junction proteins and express mesenchymal markers like fibronectin, Vimentin and $\mathrm{N}$-cadherin. This leads the cells to undergo morphological changes, lose cell-cell adhesion properties and become elongated. ASXL1 knockdown in MCF10A using siASXL1 transfection resulted in mesenchymal-like sporadic long spindle phenotype compared to sicontrol transfected cells with intact ASXL1 (Fig. 9A). Based on RNA-seq data, several epithelial and mesenchymal genes involved in EMT were selected for further validation. For this, gene expression analysis for EMT markers was performed by qRT-PCR and the results were depicted as "relative mRNA expression". As expected, the epithelial markers (CDH1, TJP3) were significantly down-regulated while the mesenchymal markers $(\mathrm{CDH} 2, \mathrm{VIM}, \mathrm{FN} 1)$ were significantly upregulated in ASXL1 depleted cells compared to siControl transfected cells. We also checked the expression of CD24, a marker associated with a differentiated phenotype, which was reported to be absent in cancer stem-like cells (Al-Hajj, Wicha et al. 2003) (Ponti, Costa et al. 2005). In support of the other data, CD24 expression was significantly decreased upon ASXL1 depletion. Furthermore, DLL1, a ligand for Notch signaling was significantly up-regulated in ASXL1 depleted cells (Fig. 9B \& C). These results suggest that ASXL1 is required for the expression of epithelial genes and for suppression of mesenchymal genes in basal-like mammary epithelial cells. 
A

\section{MCF10A}

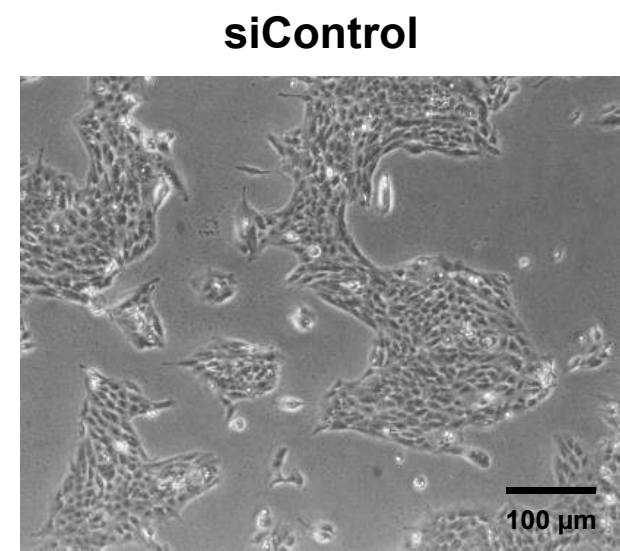

siASXL1

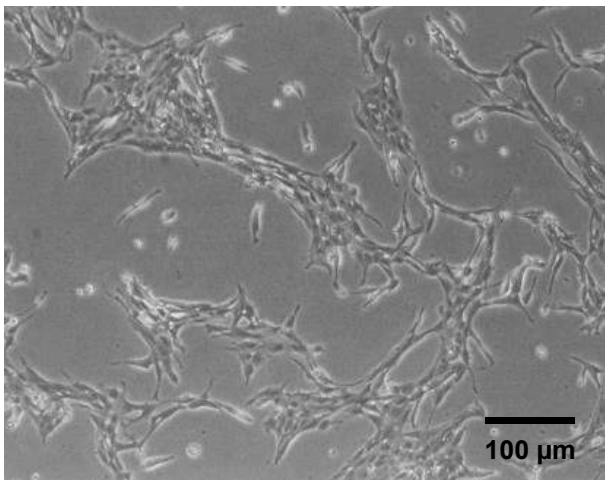

B
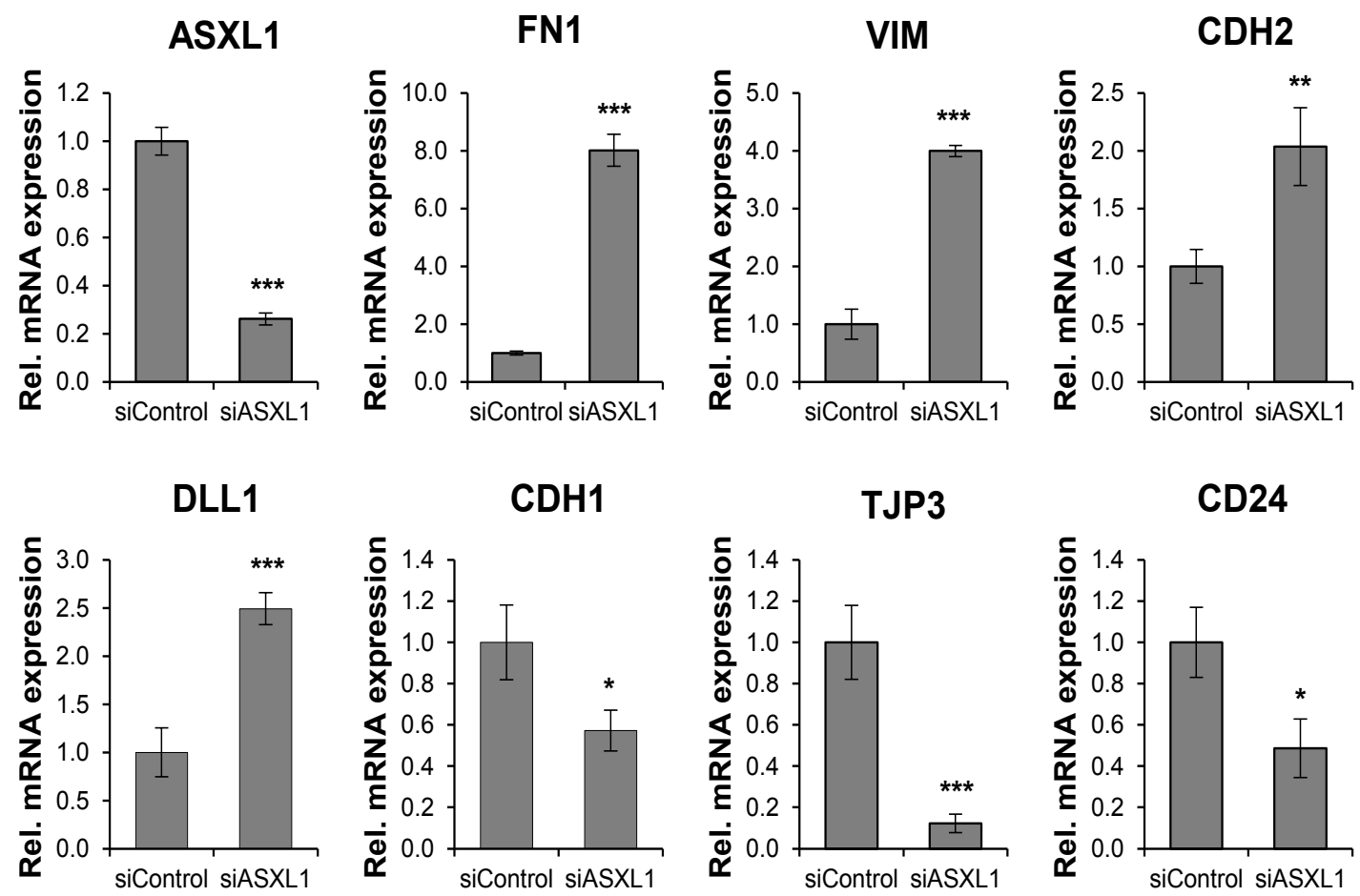
C

ASXL1

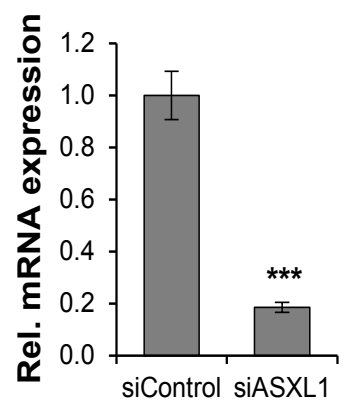

DLL1

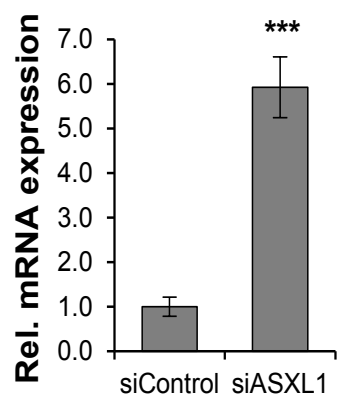

FN1

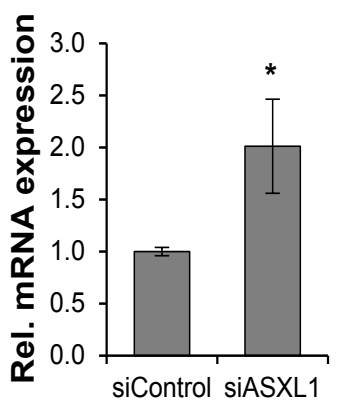

CDH1

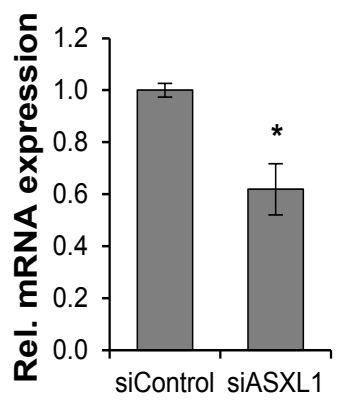

VIM

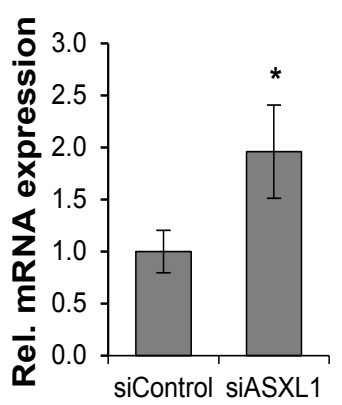

TJP3

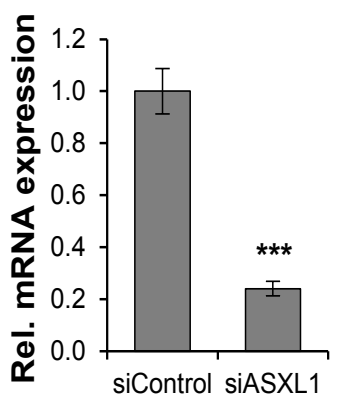

$\mathrm{CDH} 2$

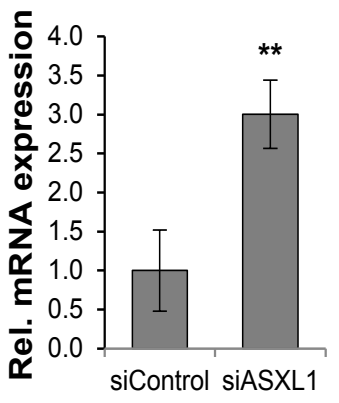

CD24

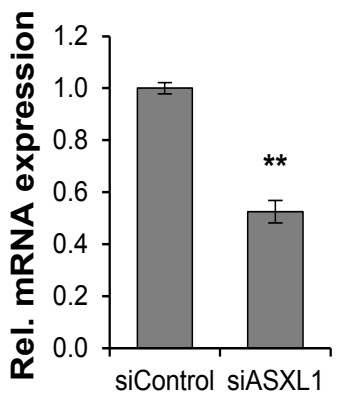

Figure. 9: Depletion of ASXL1 induces EMT phenotype. (A) Phase contrast images exhibiting the morphological changes of MCF10A cells upon ASXL1 knockdown. Scale bars are represented as $100 \mu \mathrm{m}$. (B) Gene expression levels of epithelial markers (CDH1 and TJP3) and mesenchymal markers (CDH2, FN1 and VIM) were analyzed by qRT-PCR in (B) MCF10A and (C) MCF12A cells and represented as "relative mRNA expression" normalized to HNRNPK expression level. Data are shown as mean \pm SD. $n=3 .{ }^{* * *} p \leq 0.005,{ }^{* *} p \leq 0.01,{ }^{*} p$ $\leq 0.05$.

We also checked the expression of EMT markers by Western blot following the depletion of ASXL1 in MCF10A and MCF12A cells. Consistent with our gene expression analysis, ASXL1 depletion resulted in upregulation of mesenchymal markers, Vimentin, N-cadherin and ZEB1 and downregulation of epithelial markers, E-cadherin and ZO1 (Fig. 10A). To further support the findings of EMT marker's gene expression at RNA and protein level in ASXL1 depleted cells, we visualized selected EMT markers, by immunofluorescence staining and confocal microscopy. For this, we used MCF10A cells transfected with siControl and siASXL1 and stained with antibodies for the epithelial marker, E-cadherin and mesenchymal marker, 
Vimentin $72 \mathrm{~h}$ post transfection. Confocal analyses of the immunofluorescence staining revealed a significant downregulation of the epithelial marker, E-cadherin and a strong upregulation of the mesenchymal marker, Vimentin in ASXL1 depleted cells (Fig. 10B). Taken together, these results revealed the involvement of ASXL1 in suppressing EMT.

A
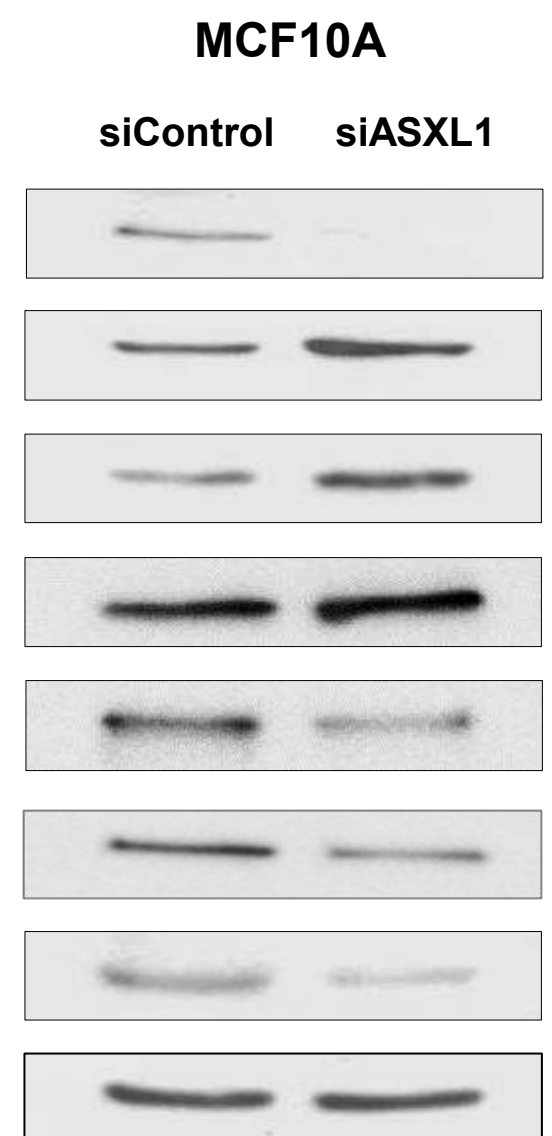

MCF12A

siControl siASXL1

ASXL1

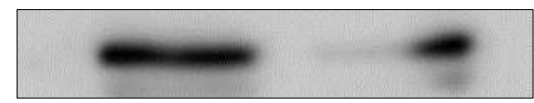

Vimentin

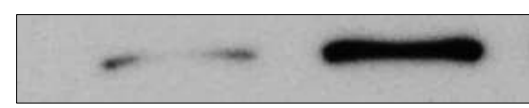

$\mathrm{CDH} 2$

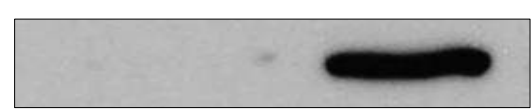

ZEB1

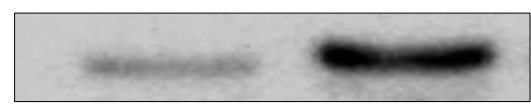

Z01

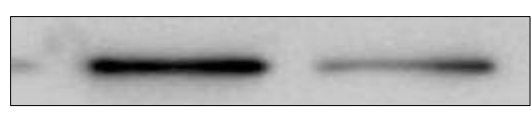

CDH1

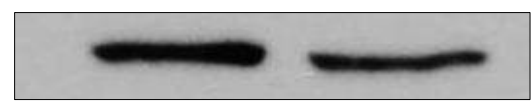

CD24

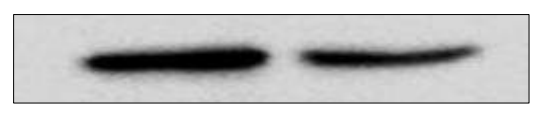

HSC70

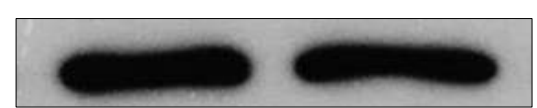


B
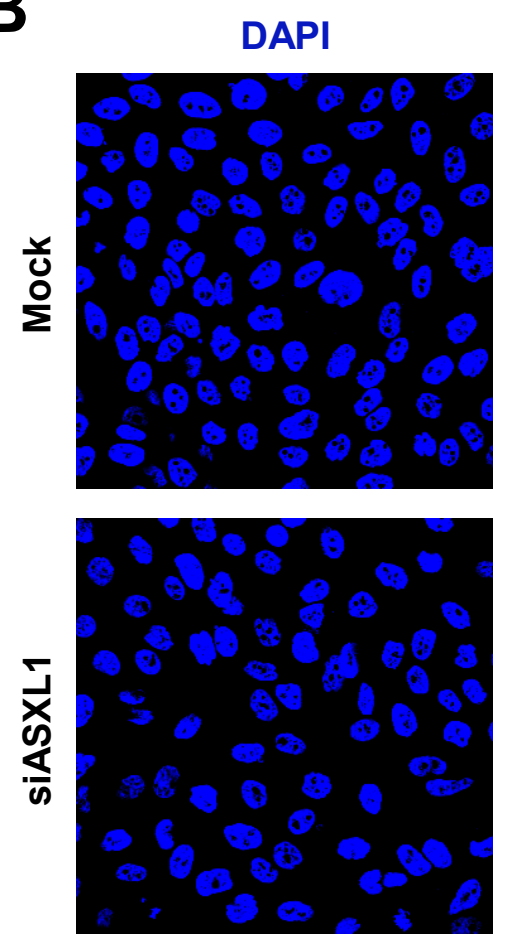

\section{DAPI}
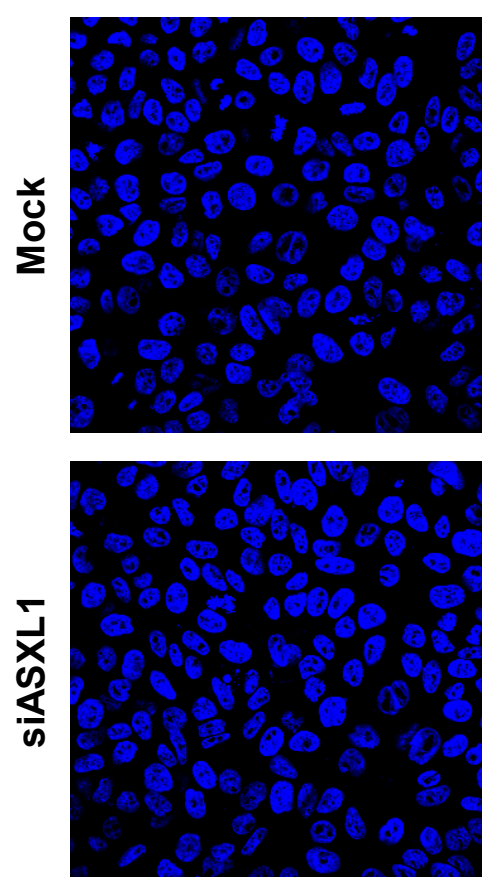

CDH1
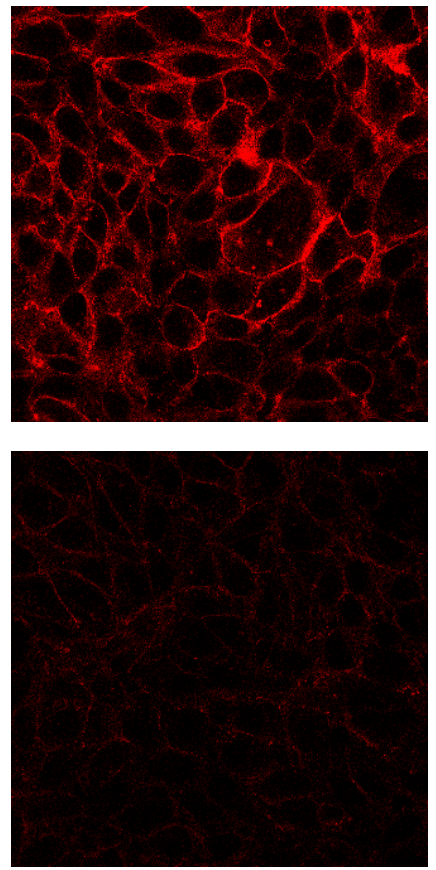

Vimentin
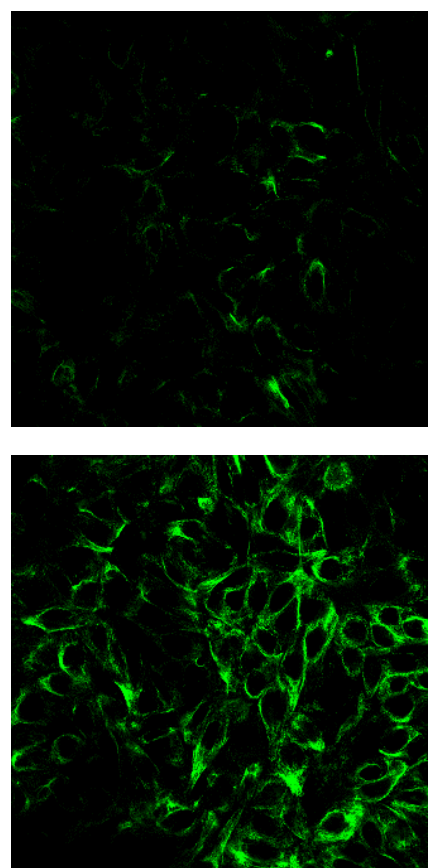

MERGE

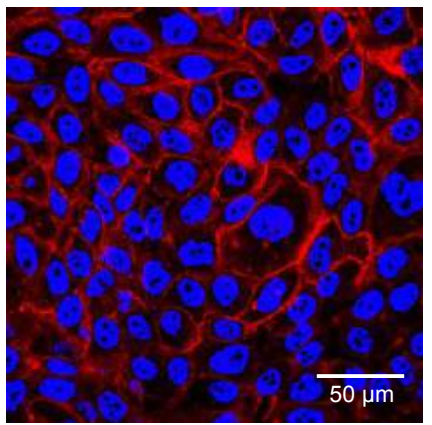

$50 \mu \mathrm{m}$

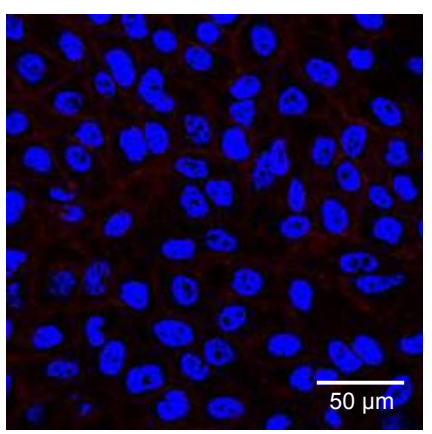

MERGE
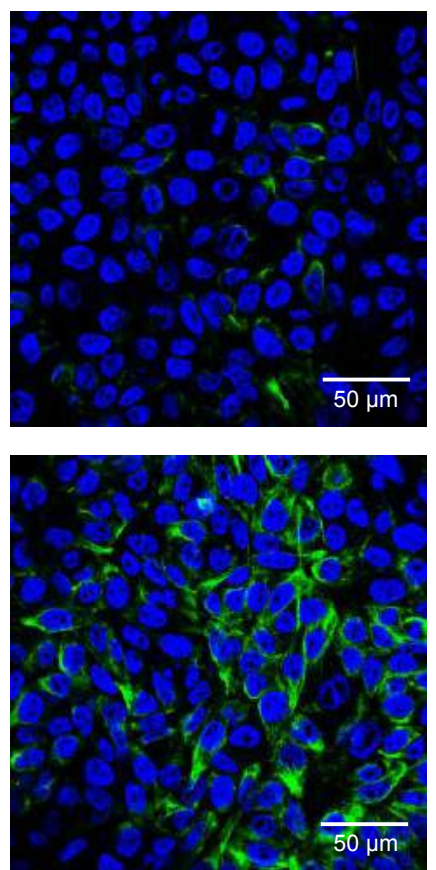

Figure. 10: ASXL1 loss enhances EMT phenotype. (A) Immunoblotting analysis of whole cell lysates depicting decreased expression of epithelial markers (E-cadherin and ZO1) and increased expression of mesenchymal markers ( $N$-cadherin, Vimentin and ZEB1) upon loss of ASXL1 in MCF10A and MCF12A cells. ASXL1 immunoblot shows the knockdown efficiency. HSC70 was used as a loading control. (B) Immunofluorescence staining of epithelial marker, E-cadherin and mesenchymal marker. Vimentin in ASXL1 depleted cells 
indicates enhanced EMT. DAPI was used to stain the nuclei. Scale bars are represented as $50 \mu \mathrm{m}$.

\subsubsection{ASXL1 depletion enhances migratory properties}

In cancer cells, acquiring EMT phenotype is the foremost step for migration which helps to transform differentiated cells into the stem-cell-like state (Mani, Guo et al. 2008) (Polyak and Weinberg 2009). One of the main features of EMT is to increase the migratory potential of cells to travel to distant places to develop a secondary tumor (metastasis). The earlier results revealed that the loss of ASXL1 enhances EMT-like phenotype. To understand whether this in turn enhances the migratory potential of the cells, we performed a transwell migratory assays in MCF10A and MCF12A cells. For this, the cells were transfected with either control or ASXL1 siRNAs and grown for 48 hours. After 48 hours, cells were seeded into $8.0 \mu \mathrm{m}$ PET track-etched membrane cell inserts and allowed to migrate through the membrane for further 48 hours. Finally, the migrated cells on the membrane were visualized by crystal violet staining, which revealed that ASXL1 depletion leads to enhanced migratory potential compared to the control cells (Fig. 11). Together, these findings demonstrate that ASXL1 depletion is associated with migration. 

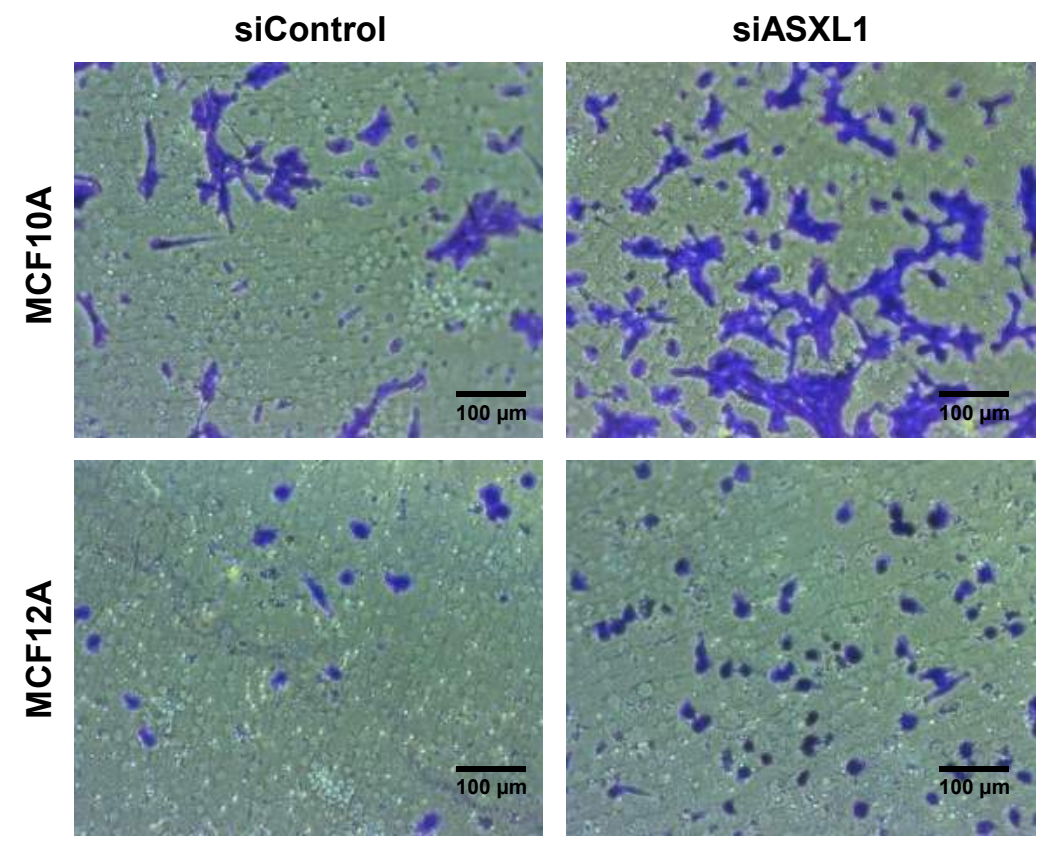

Figure. 11: Loss of ASXL1 promotes migratory potential. MCF10A and MCF12A cells were used for the transwell migration assay. Cells transfected with siASXL1 and siControl were seeded as single cells $(50,000)$ into the upper Chamber of the insert after 48 hours. Cells are allowed to migrate for additional 48hrs and fixed with $100 \%$ methanol. The membrane was stained by using crystal violet. There is an increased migration in ASXL1 depleted in MCF10A and MCF12A cells. Scale bars are represented as $100 \mu \mathrm{m}$.

\subsubsection{Loss of ASXL1 induces stem cell characteristics}

In order to identify the enrichment of stem cell populations in vitro, the mammospheres culture in breast cancer has been widely used (Grimshaw, Cooper et al. 2008). The cells transfected with Control and ASXL1 siRNAs were seeded as single cells into low attachment plates and allowed to grow in non-adherent and nondifferentiating conditions. Therefore, single cells possessing stem cell properties could give rise to $3 \mathrm{D}$ spheres called mammospheres the number of which were later counted. ASXL1 depletion significantly increased the formation of the number of mammospheres (Fig. 12A \& B) which suggests an important role of ASXL1 in suppressing stem cell phenotype and maintaining epithelial phenotype. 
A
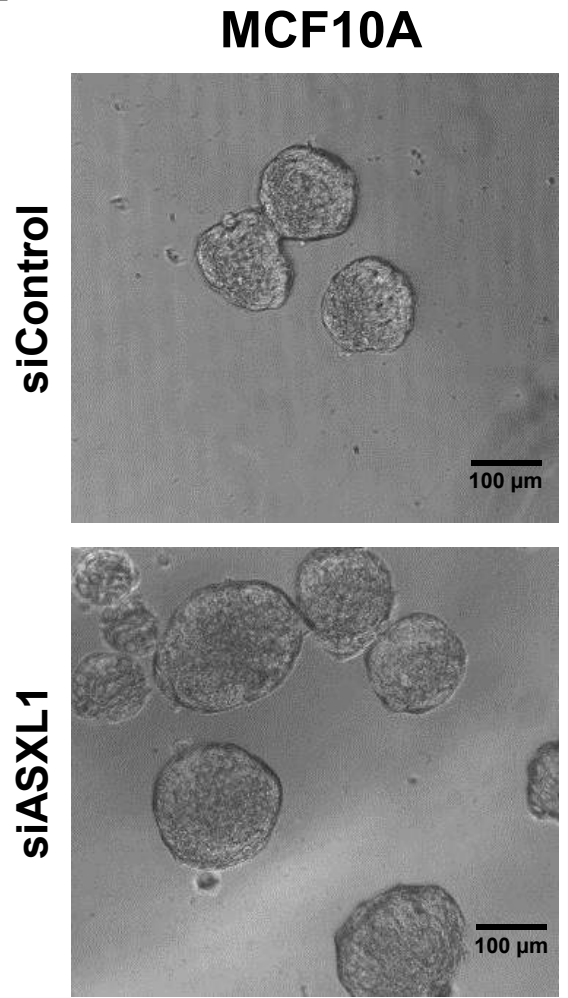

B

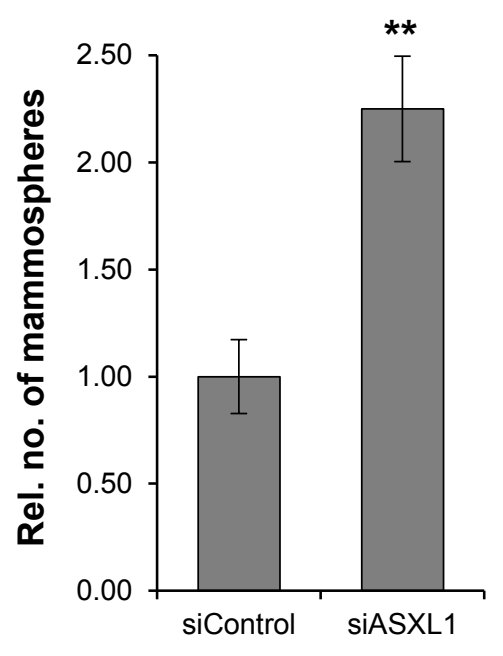

Figure. 12: ASXL1 depletion induces stem-cell properties. (A) Phase contrast images of mammospheres upon control and ASXL1 siRNAs transfection in MCF10A cells. 10x magnification. Scale bars are represented as $100 \mu \mathrm{m}$. (B) The mammospheres formed were quantified and represented as "relative number of mammospheres". The data indicated that there is an increase in mammospheres number upon ASXL1 knockdown. The data are represented as \pm SD. $n=3 .{ }^{* \star *} p \leq 0.005,{ }^{* *} p \leq 0.01,{ }^{*} p \leq 0.05$.

\subsection{Genome-wide occupancy of ASXL1}

Our results demonstrated that ASXL1 plays an important role in suppressing EMT phenotype in normal breast epithelial cells, which was supported by our transcriptome-wide analysis. However, additional studies are needed to further understand and characterize the functional and mechanistic role of ASXL1 in EMT during tumor progression and metastasis. Genome-wide occupancy studies were performed to understand the mechanism of action and determine the vital target genes of ASXL1. For this, we performed chromatin immunoprecipitation followed by deep sequencing (ChIP-seq). 


\subsubsection{Enrichment of ASXL1 on promoter regions}

ChIP-sequencing of ASXL1 was performed in MCF10A to investigate the occupancy of ASXL1 on specific genome locations like promoters, introns and coding exons using a tool, CEAS (Cis-regulatory Annotation System). CEAS is a part of Cistrome package which is used to determine the relative enrichment of ChIP-binding regions at specific genome regions with respect to the whole genome. CEAS data pie chart depicts the distribution of ASXL1 enrichment in various genomic regions. CEAS analysis revealed that the majority of ASXL1 binding sites $(70.2 \%)$ were confined to the distal intergenic regions compared to the whole genome (Fig. 13A \& B). CEAS also revealed that a significant portion ( $8 \%$ and $9.5 \%$, respectively) of ASXL1 binding sites were confined to promoter regions and introns compared to the whole genome. A small portion (4.8\%) of binding sites was confined to coding exons. Furthermore, aggregate plot analysis was performed to observe the ASXL1 enrichment around the transcription start site (TSS) of the ASXL1 bound genes, which revealed that the highest ASXL1 enrichment near the TSS region of the genes and a low signal at the $5 \mathrm{~kb}$ upstream and downstream stream of the TSS (Fig. 13C). Taken together these results show that ASXL1 is mostly recruited to promoter and distal intergenic regions of target genes genome-wide. Furthermore, a very few ASXL1 binding sites were enriched on introns and coding exons. 
A

\section{Genome}

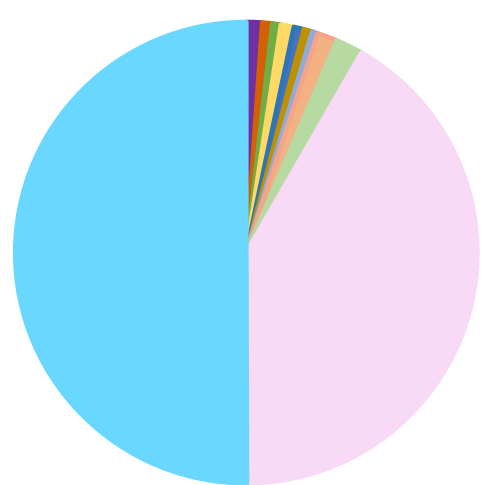

\begin{tabular}{|l|l|c|c|}
\hline & & Genome & ChIP \\
\hline$\square$ & Promoter (<=1000 bp) & 1.1 & 8 \\
\hline$\square$ & Promoter (1000-2000 bp) & 0.7 & 1.4 \\
\hline$\square$ & Promoter (2000-3000 bp) & 0.6 & 0.7 \\
\hline$\square$ & Downstream (<=1000 bp) & 0.9 & 0.6 \\
\hline$\square$ & Downstream (1000-2000 bp) & 0.7 & 0.7 \\
\hline$\square$ & Downstream (2000-3000 bp) & 0.6 & 0.5 \\
\hline$\square$ & 5'UTR & 0.4 & 2.4 \\
\hline$\square$ & 3'UTR & 1.4 & 1.2 \\
\hline$\square$ & Coding exon & 1.9 & 4.8 \\
\hline$\square$ & Intron & 41.6 & 9.5 \\
\hline$\square$ & Distal intergenic & 50 & 70.2 \\
\hline
\end{tabular}

\section{ChIP}

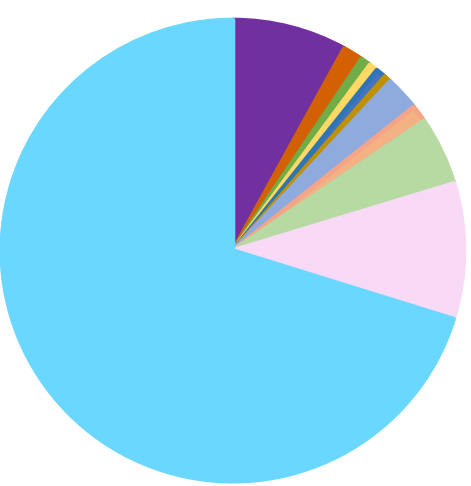

7

6

2

9.5

B
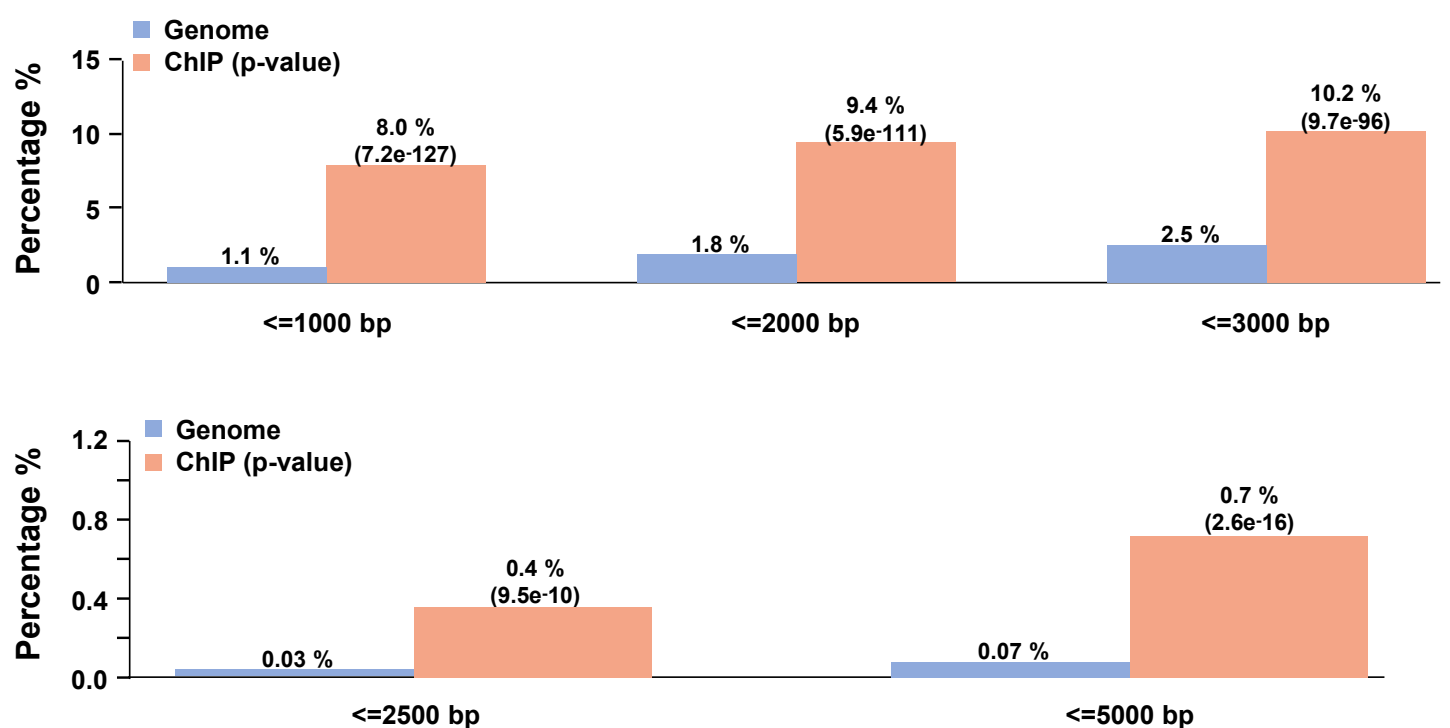
C

\section{Average Profile of ASXL1 around the Centre of Sites}

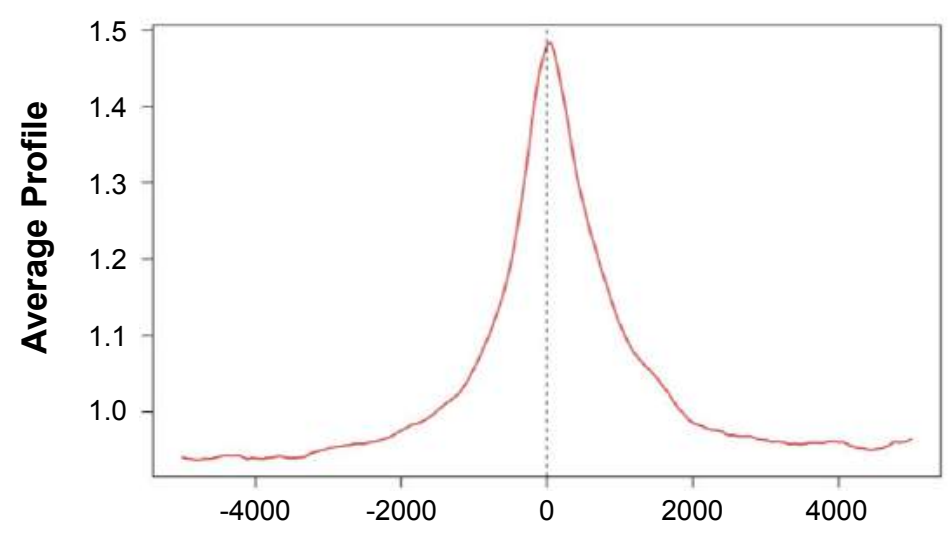

Relative Distance from the Centre (bp)

Figure. 13: ASXL1 enrichment in the genome. (A) Pie chart depicting the relative occupancy of ASXL1 on various genomic regions with respect to the whole genome. (B) Percentage enrichment of ASXL1 enrichment regions around the promoter relative to the genome. (C) Aggregate plot showing average ASXL1 enrichment $\pm 5 \mathrm{~kb}$ around the transcriptional start site (TSS).

\subsubsection{ASXL1 occupancy on individual genomic regions}

To examine the mechanistic role of ASXL1 in EMT, ChIP-seq analysis was performed in MCF10A cells. Genome-wide and single gene analyses show that ASXL1 largely occupies regions of the transcription start site (TSS). Remarkably, we observed a prominent ASXL1 peak around the promoter regions of ASXL1 affected genes (PTEN, TJP3 and ARID1B) (Fig. 14A). To confirm whether ASXL1 indeed directly bind to the above genes, we designed primers across the TSS, for ChIPqRT-PCR analysis, where the ASXL1 peak was visualized. And, ASXL1 ChIP was performed in MCF10A cells with or without ASXL1 depletion followed by the confirmation of ASXL1 enrichment using ChIP-qRT-PCR. Consistent with the ChIPseq results, ChIP-qRT-PCR analysis of ChIP samples showed a significant enrichment of ASXL1 binding on the TSS region of PTEN, TJP3 and ARID1B genes. 
Furthermore, the ChIP-qRT-PCR analysis showed a significant decrease of ASXL1 binding on the TSS upon ASXL1 depletion, which proves the specificity of ASXL1 binding (Fig. 14B).

A

\section{ASXL1}
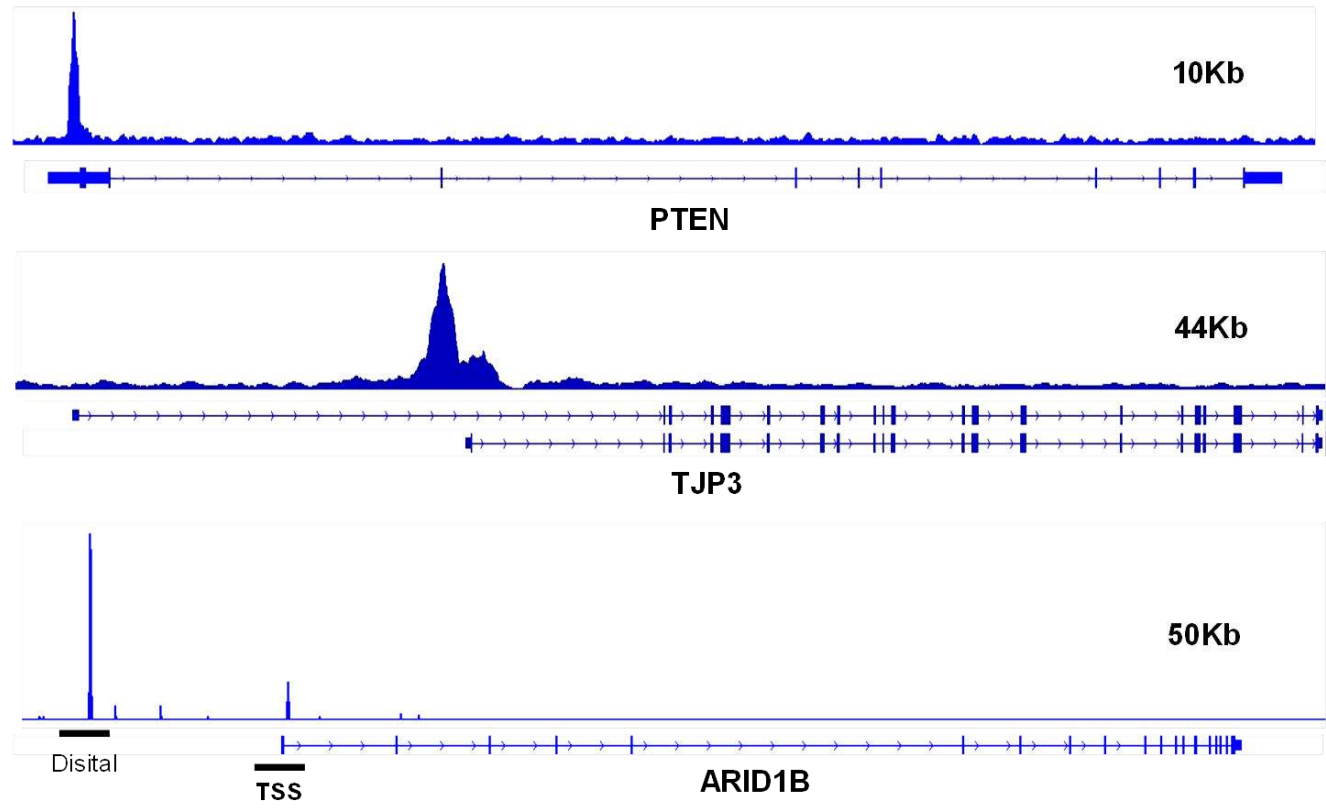

B
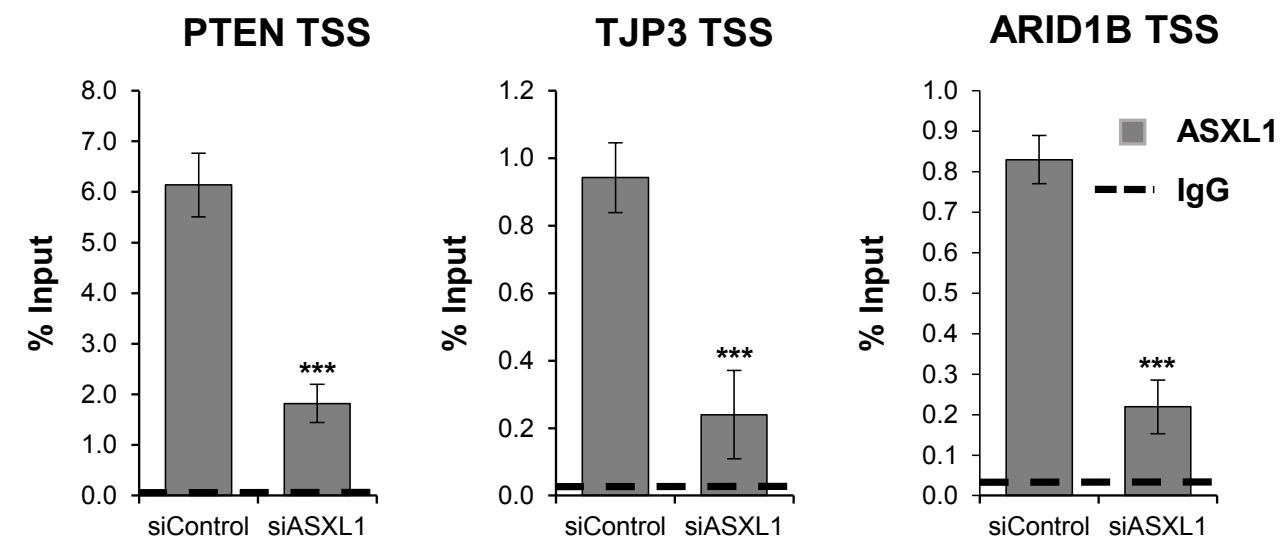

Figure. 14: ASXL1 enrichment on the promoters of single genes. (A) ChIP-seq profile of ASXL1 on PTEN, TJP3 and ARID1B genes. Significant peak was observed around the TSS. (B) ChIP analysis of ASXL1 enrichment on TSS of single genes in MCF10A cells with and without ASXL1 depletion. Immunoprecipitated DNA is compared to input and represented as 
"\% of Input". IgG antibody was used a negative control to measure the background signal level and displayed as a dotted line. Data are shown as mean \pm SD. $n=3$.

\subsection{ASXL1 occupancy correlate with gene expression}

To determine whether ASXL1 play a direct role in regulating transcription of its target genes, the expression of these genes was studied in presence or absence of ASXL1. For this, MCF10A and MCF12A cells were transfected with siControl or siASXL1 followed by RNA isolation $72 \mathrm{~h}$ post transfection and cDNA preparation. This cDNA was used to assess the changes in expression of PTEN, TJP3 and ARID1B mRNA levels by qRT-PCR, which revealed that there was a significant decrease in gene expression of PTEN, TJP3 and ARID1B upon ASXL1 depletion in both MCF10A and MCF12A cells (Fig. 15A \& B). These results confirm that ASXL1 directly regulates the expression of PTEN, TJP3 and ARID1B.

A

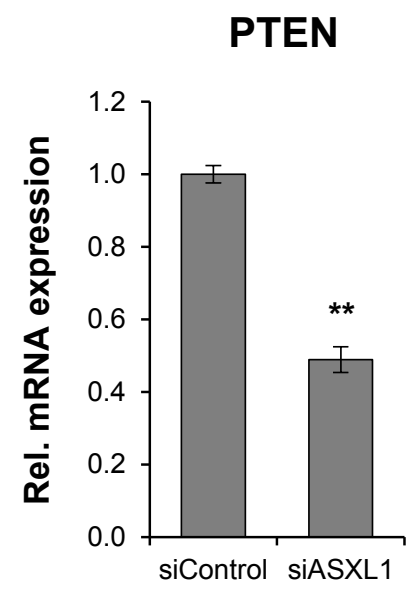

MCF10A

TJP3

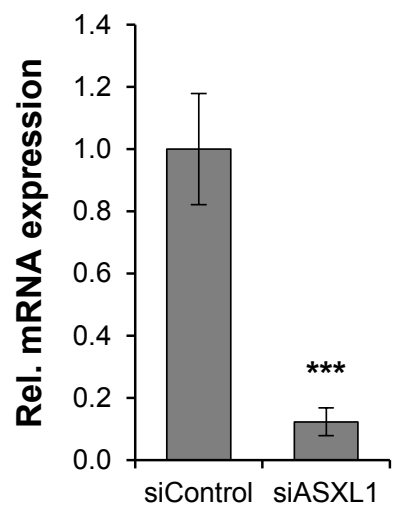

ARID1B

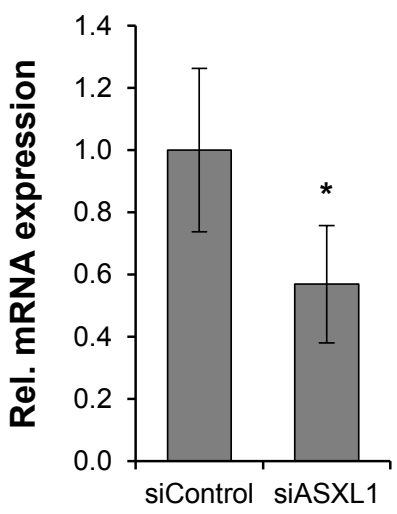


B

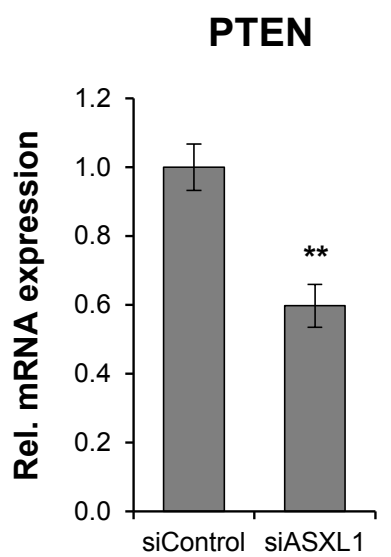

MCF12A

TJP3

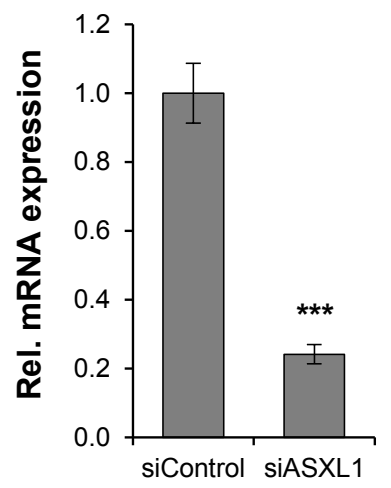

ARID1B

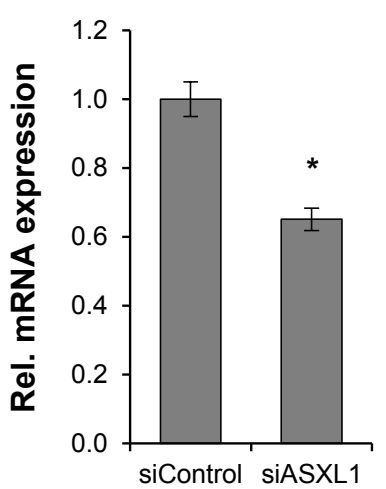

Figure. 15: ASXL1 directly regulates gene expression. Gene expression levels of ASXL1 bound genes (PTEN, TJP3 and ARID1B) were analyzed by qRT-PCR in (A) MCF10A and (B) MCF12A cells and represented as "relative mRNA expression" normalized to HNRNPK expression level. The qRT-PCR reveals that there is a significant decrease in expression of genes in ASXL1 depleted cells. Data are shown as mean \pm SD. $n=3 .{ }^{* * *} p \leq 0.005,{ }^{* *} p \leq 0.01$, $p \leq 0.05$.

\subsection{Generation of ASXL1 expressing stable cell line}

To further validate the role of ASXL1 in EMT, MCF10A (normal breast epithelial cells) and MDA-MB231 (human breast cancer, adenocarcinoma) cells were stably transfected with linearized expression vector for ASXL1 or control vector and grown in Hygromycin selection medium for a few weeks. These stable cells were utilized for further studies.

\subsubsection{Ectopic expression of ASXL1 reverses EMT phenotype}

To reveal the effect of ASXL1 expression on EMT phenotype, we performed several cell culture based assays in the stable cell lines expressing ASXL1. As already shown, ASXL1 depletion results in downregulation of epithelial markers and upregulation of mesenchymal markers. We then checked whether overexpression of ASXL1 can reverse the effects of ASXL1 depletion i.e. increase in the expression of 
epithelial markers and decrease in the expression of mesenchymal markers in MCF10A and MDA-MB-231 cells. For this, we isolated RNA and protein samples from the stable cell lines. The gene expression levels were analyzed by qRT-PCR and depicted as "relative mRNA expression". The epithelial markers (CDH1, TJP3) were significantly up-regulated and mesenchymal markers (CDH2, VIM, FN1) were significantly down-regulated in ASXL1 expressing stable cells compared to the control cells. Furthermore, the genes bound by ASXL1 were also regulated by ASXL1 overexpression (TJP3, PTEN and ARID1B) (Fig.16A \& B).

A
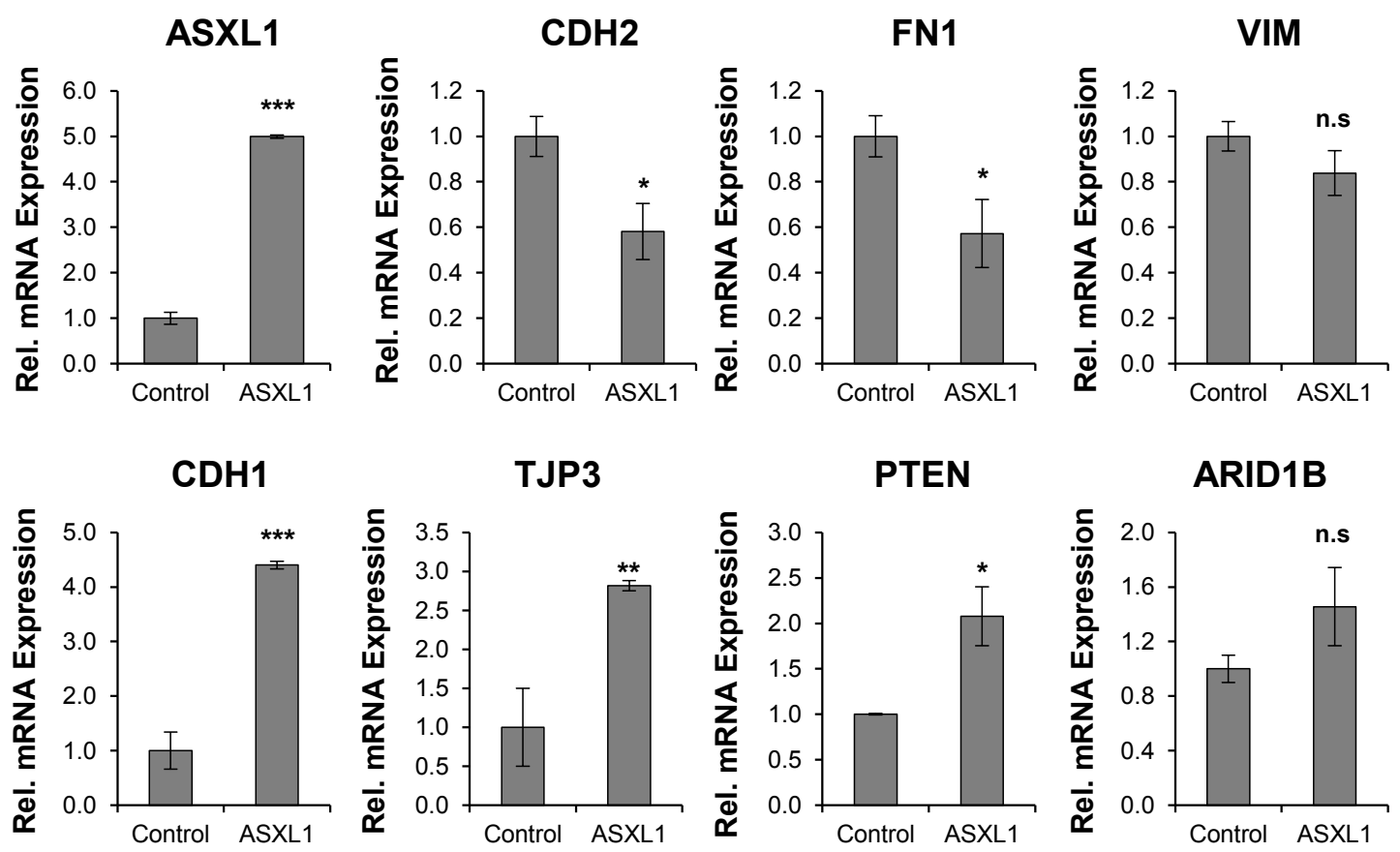
B
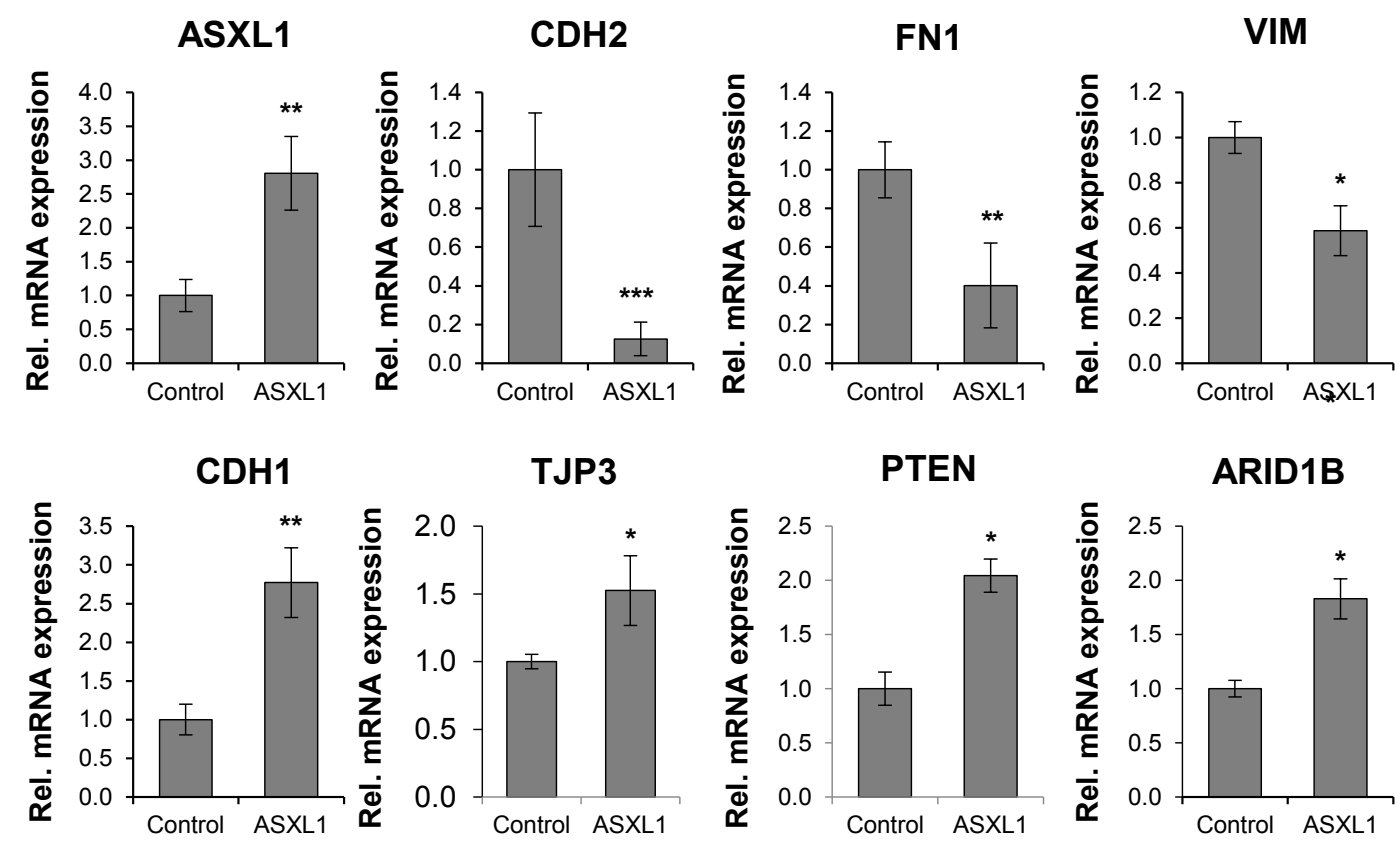

Figure. 16: Expression of ASXL1 induces MET phenotype. (A) Gene expression levels of epithelial markers (CDH1 and TJP3) and mesenchymal markers (CDH2, FN1 and VIM) were analyzed by qRT-PCR in (A) MCF10A and (B) MDA-MB-231 cells and represented as "relative mRNA expression" normalized to HNRNPK or 36B4 expression level. Data are shown as mean $\pm \mathrm{SD}$. $\mathrm{n}=3 .{ }^{* *} \mathrm{p} \leq 0.005,{ }^{* *} \mathrm{p} \leq 0.01,{ }^{*} \mathrm{p} \leq 0.05$.

To further support our findings from qRT-PCR data in the stable MCF10A and MDAMB-231 cells, the changes in EMT markers expression were analyzed by Western blot analysis. It was observed that epithelial markers, E-cadherin and ZO1 were upregulated and mesenchymal markers, Vimentin and $\mathrm{N}$-cadherin were downregulated (Fig. 17). Altogether, these results demonstrate that ASXL1 suppresses EMT and promotes mesenchymal-to-epithelial (MET) phenotype. 
MCF10A

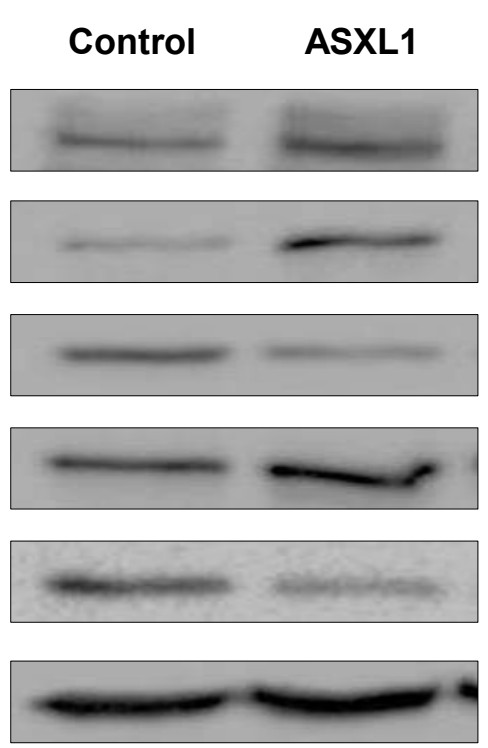

MDA-MB-231

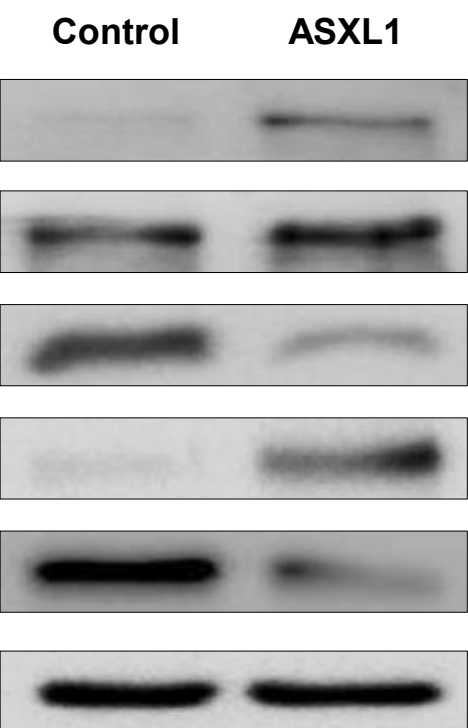

Figure. 17: ASXL1 expression reverses EMT phenotype. Immunoblotting analysis of whole cell lysates depicting increased expression of epithelial markers (E-cadherin and ZO1) and decreased expression of mesenchymal markers (N-cadherin, Vimentin and ZEB1) upon ectopic expression of ASXL1 in MCF10A and MDA-MB-231 cells. ASXL1 immunoblot shows the increase in ASXL1 expression. HSC70 was used as a loading control.

\subsubsection{ASXL1 expression reduces migratory properties}

From the earlier results, we demonstrated that ectopic expression of ASXL1 reverses EMT-like phenotype whereas ASXL1 depletion promotes it. To understand whether an ectopic expression can inhibit the cell migration, as it is one of the important characteristics of EMT or metastasis, we performed transwell migratory assays in MCF10A and MDA-MB-231 cells, stably transfected with ASXL1 expressing plasmid. For this the cells were trypsinized and 50,000 MCF10A and 15,000 MDA-MB-231 cells each were seeded into $8.0 \mu \mathrm{m}$ PET track-etched membrane cell inserts and allowed to migrate through the membrane for 48 hours. Visualization by crystal violet staining of the membrane revealed that ASXL1 overexpression leads to a reduced migratory potential compared with the control cells (Fig. 18). 

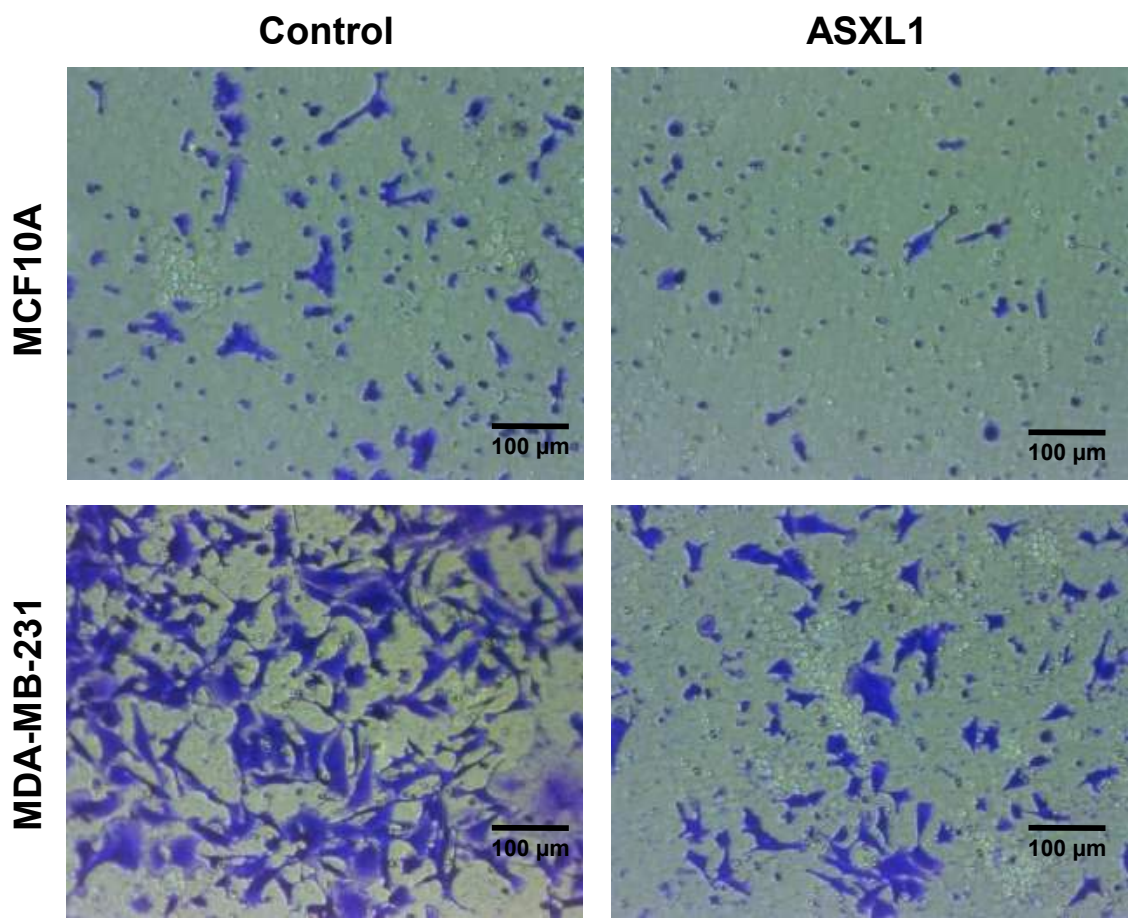

Figure. 18: Expression of ASXL1 suppresses migratory potential. MCF10A and MDAMB-231 stable cells were used for the transwell migration assay. Stable cell lines, MCF10A $(50,000)$ and MDA-MB-231 $(15,000)$ were seeded as single cells into the upper Chamber of the insert. Cells are allowed to migrate for $48 \mathrm{hrs}$ and fixed with $100 \%$ methanol. The membrane was stained by using crystal violet. There is decreased migration in ASXL1 overexpressed (A) MCF10A and (B) MDA-MB-231 cells. Scale bars are represented as 100 $\mu \mathrm{m}$.

\subsubsection{ASXL1 expression reduces stem cell characteristics}

To understand the role of ASXL1 on stem cell phenotype, the stable cell line, MCF10A was seeded as single cells into low attachment plates and allowed to grow in non-adherent and non-differentiating conditions. The mammospheres formed were counted and observed that the number of mammospheres were reduced upon ASXL1 overexpression which demonstrates that ASXL1 negatively regulates the mammospheres formation and anchorage-independent growth by suppressing the stem cell phenotype. (Fig. 19A \& B). 
A

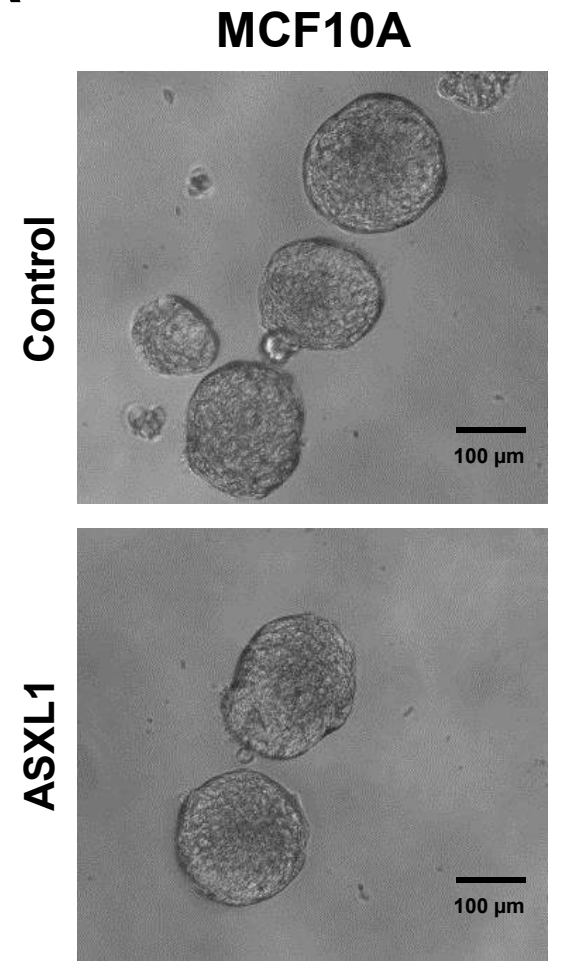

B

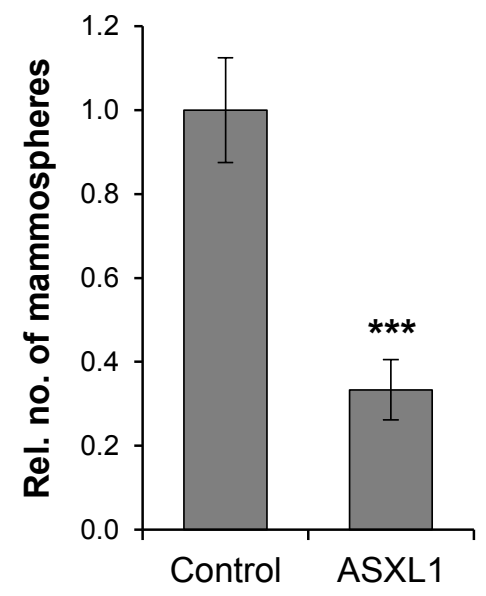

Figure. 19: Expression of ASXL1 depletes stem-cell properties. (A) Phase contrast images of mammospheres upon control and ASXL1 expressing stable cells (MCF10A). The scale bars are represented as $100 \mu \mathrm{m}$. (B) The mammospheres formed were quantified and represented as "relative number of mammospheres". The data indicated that there is a decrease in mammospheres number upon ASXL1 overexpression. The data are represented as $\pm S D . n=3 .{ }^{* \star *} p \leq 0.005,{ }^{* *} p \leq 0.01,{ }^{*} p \leq 0.05$.

\subsection{ASXL1 occupancy increased with ectopic ASXL1 expression}

To investigate the effect of ASXL1 expression on ASXL1 occupancy, ASXL1 ChIP was performed in MDA-MB-231 cells with ASXL1 stable expression. ASXL1 enrichment was checked by ChIP-qRT-PCR, which showed a significant enrichment of ASXL1 occupancy on the TSS of TJP3, PTEN and ARID1B genes. Furthermore, the ChIP-qRT-PCR analysis showed a significant increase of ASXL1 binding on the TSS upon ASXL1 stable expression, which proves the specificity of ASXL1 binding (Fig. 20). 


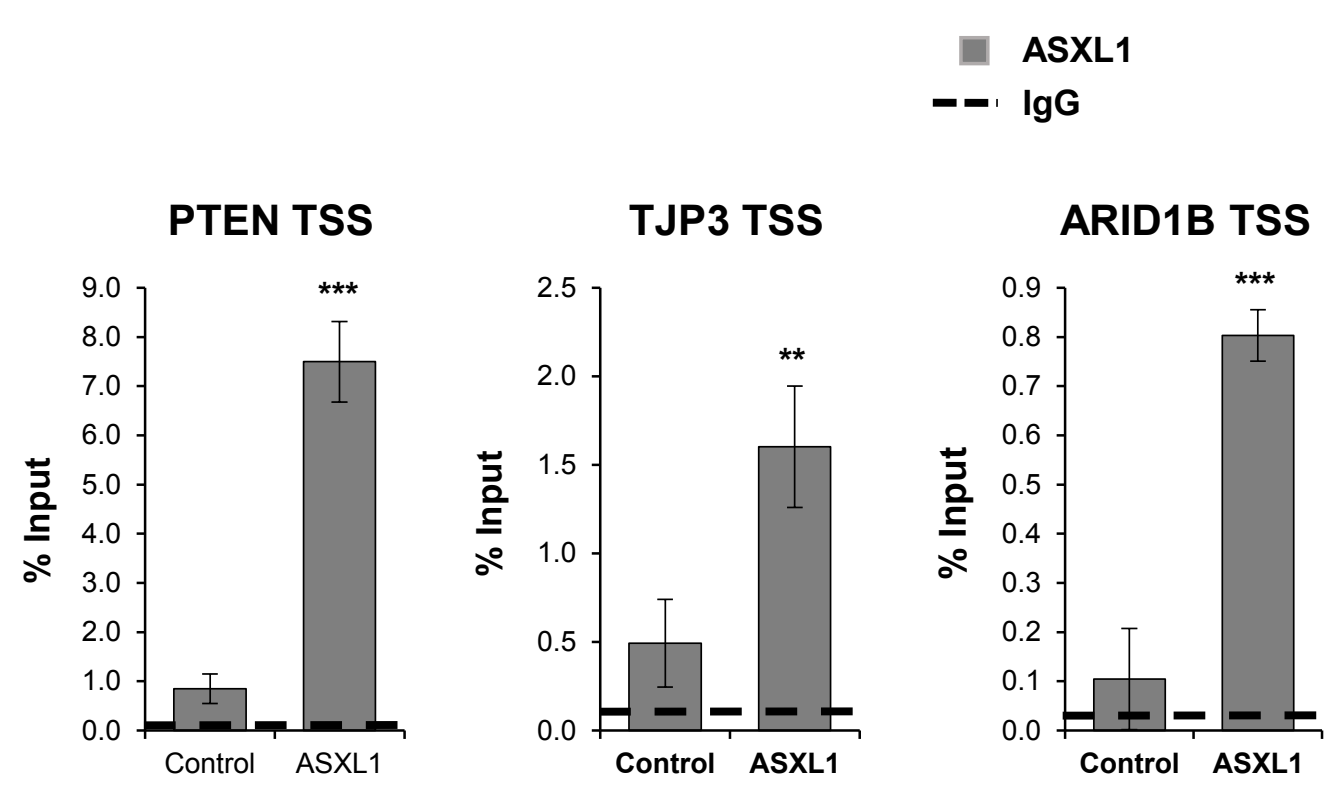

Figure. 20: Enrichment of ASXL1 increased with ectopic ASXL1 expression. ChIP analysis of ASXL1 enrichment on the promoter of PTEN, TJP3 and ARID1B genes in MDAMB-231 stable cells with and without ectopic ASXL1 expression. Immunoprecipitated DNA is compared to input and represented as "\% of Input". IgG antibody was used a negative control to measure the background signal level and displayed as a dotted line. Data are shown as mean \pm SD. $n=3$.

\subsubsection{ASXL1 regulates epithelial gene expression by promoting the expression of PTEN}

To further elucidate the mechanism by which ASXL1 promotes EMT, we examined the genes regulated following perturbation of ASXL1. We found that the expression of PTEN was significantly down-regulated by ASXL1 depletion and also up-regulated by ectopic expression of ASXL1. PTEN is a tumor suppressor and a multifunctional enzyme belonging to the phosphatase family which is reportedly mutated in several cancers and also known to regulate cell migration (Bowen, Doan et al. 2009, Fournier, Fata et al. 2009). 
A
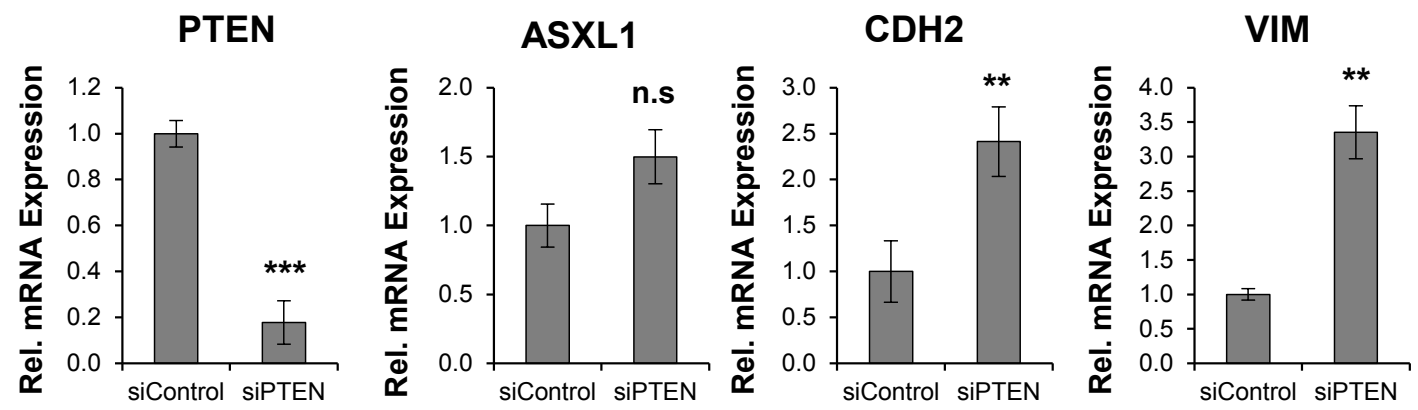

FN1

$\mathrm{CDH} 1$

TJP3
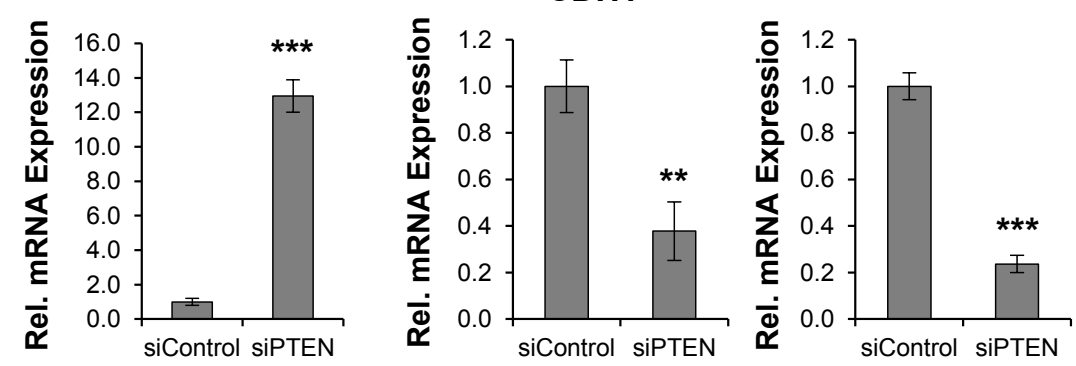

B

PTEN

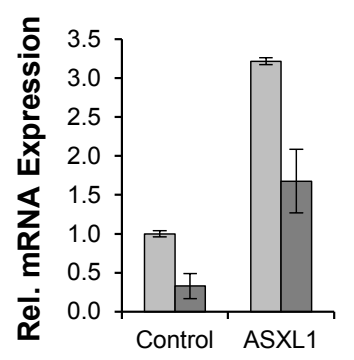

FN1

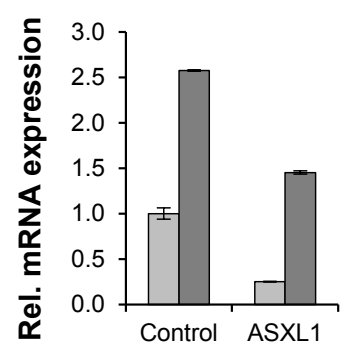

ASXL1

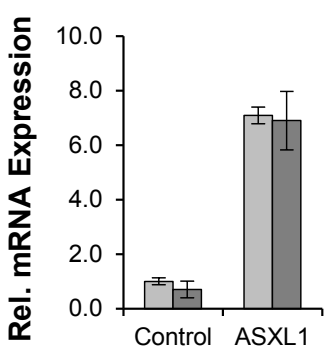

$\mathrm{CDH} 2$

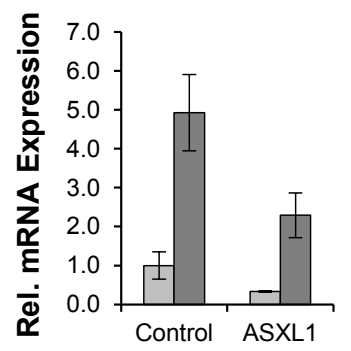

TJP3

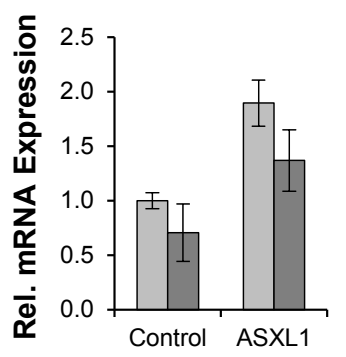

VIM

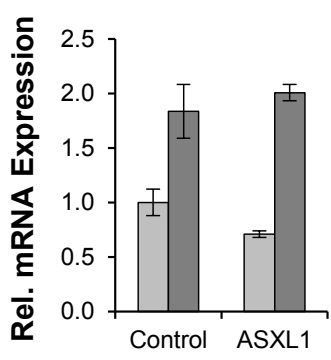

CDH1

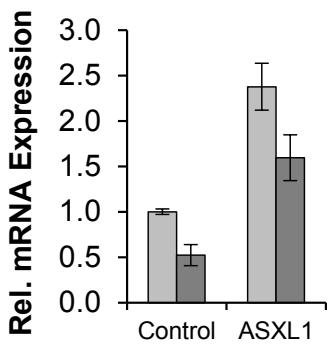

$\square$ siControl

$\square$ SIPTEN

Figure. 21: PTEN expression and its effect on EMT markers. Gene expression levels of epithelial markers (CDH1 and TJP3) and mesenchymal markers (CDH2, FN1 and VIM) were analyzed by qRT-PCR in (A) MCF10A and (B) MDA-MB-231 stable cells and represented as "relative mRNA expression" normalized to HNRNPK expression level. Data are shown as mean \pm SD. $n=3 .{ }^{* * *} p \leq 0.005,{ }^{* *} p \leq 0.01,{ }^{*} p \leq 0.05$. 
To elucidate the effect of PTEN on EMT phenotype, PTEN was depleted using siRNA in MCF10A and stable MDA-MB-231 cells followed by gene expression analysis of EMT markers using qRT-PCR. The epithelial markers, CDH1 and TJP3 were downregulated and mesenchymal markers, $\mathrm{CDH} 2, \mathrm{VIM}$, and $\mathrm{FN} 1$ were upregulated in PTEN depleted cells compared to siControl transfected cells. However, ASXL1 expression was not effected by PTEN depletion. Furthermore, the presence of ASXL1 ectopic expression in MDA-MB-231 cells was able to counteract the upregulation of mesenchymal markers, CDH2 and FN1, but not VIM (Fig. 21A and B).

To further support the role of PTEN in suppressing EMT, the changes in EMT markers were validated by Western blot analysis in PTEN depleted cells. As expected, mesenchymal markers, Vimentin and $\mathrm{N}$-cadherin were upregulated and epithelial marker, E-cadherin was downregulated (Fig. 22). Taken together, our studies showed that PTEN depletion has similar effects observed following ASXL1 loss. 
MCF10A

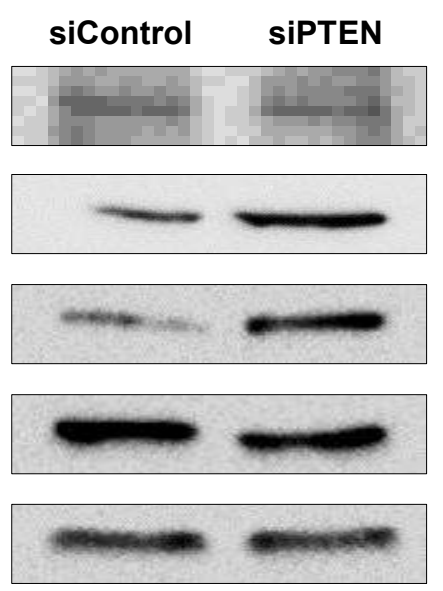

MDA-MB-231

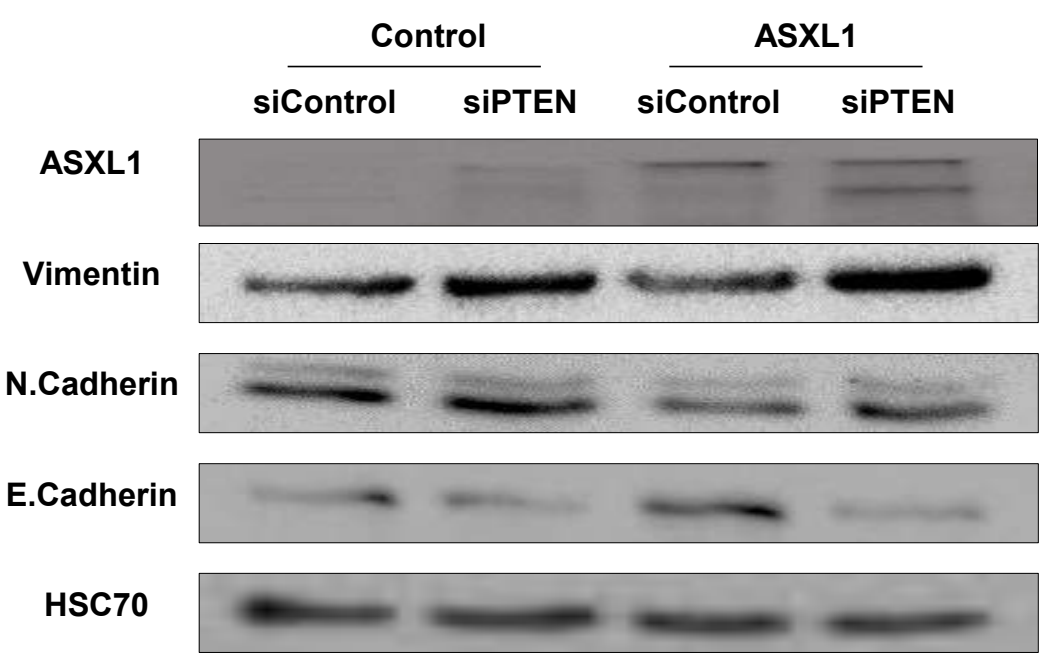

Figure. 22: ASXL1 loss enhances EMT phenotype. Immunoblotting analysis of whole cell lysates depicting decreased expression of the epithelial marker, E-cadherin and increased expression of mesenchymal markers, $\mathrm{N}$-cadherin and Vimentin upon loss of PTEN in MCF10A and MDA-MB-231 stable cells. HSC70 was used as a loading control.

\subsubsection{PTEN regulates AKT activation}

PTEN is known to inhibit the PI3K-AKT signaling pathway by catalyzing phosphatidylinositol-3, 4, 5-trisphosphate (PIP3) to phosphatidylinositol-4, 5bisphosphate (PIP2) (Cully et.al, 2006). AKT signaling is associated with the epithelial-to-mesenchymal transition, epithelial differentiation, migration and metastasis (Larue and Bellacosa, 2005; Zhuang et al., 2012). To study whether PTEN depletion is associated with activation of the PI3K-AKT signaling pathway, an immunoblot for p-AKT was performed. In MCF10A cell lines, we observed an inverse correlation between PTEN expression and p-AKT levels. Furthermore, cells with depleted expression of ASXL1 and PTEN had increased p-AKT expression whereas cells with ASXL1 expression had decreased p-AKT expression (Fig. 23). With this, our results indicate that the depletion of ASXL1 and PTEN may lead to activation of PI3K-AKT pathway, which in turn promotes epithelial-to-mesenchymal transition. 
MCF10A

siControl siASXL1 SIPTEN

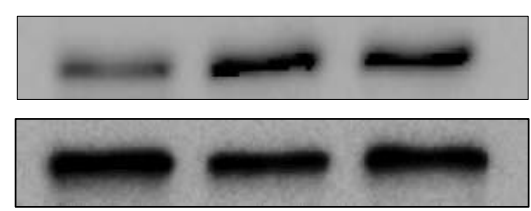

MDA-MB-231

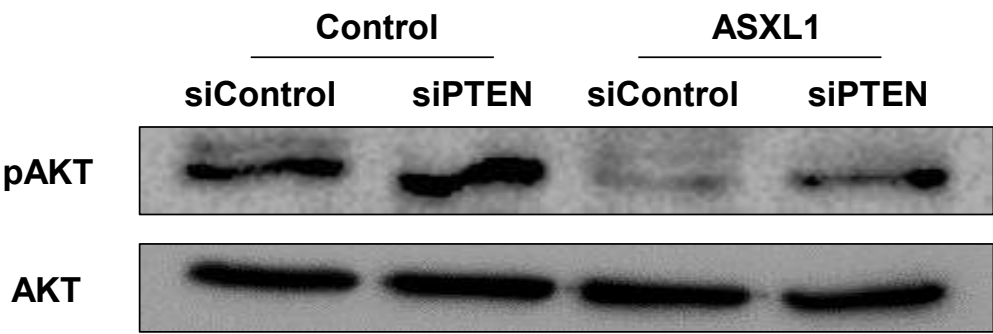

Figure. 23: PTEN depletion enhances AKT activation. Immunoblotting analysis of whole cell lysates depicting increased expression of p-AKT upon loss of ASXL1 and PTEN in MCF10A and decreased expression of p-AKT in ASXL1 expressing MDA-MB-231 stable cells. AKT was used as a loading control.

\subsection{Depletion of ASXL1 regulates global levels of epigenomes}

Previously, it was shown that ASXL1 binds to the polycomb repressive complex 2 (PRC2) members, specifically EZH2, EED, and SUZ12 and ASXL1 depletion inhibits trimethylation of $\mathrm{H} 3 \mathrm{~K} 27$ (H3K27me3). ASXL1 also associates with the H2A deubiquitinating enzyme BAP1 (Abdel-Wahab, Adli et al. 2012, Sahtoe, van Dijk et al. 2016). Thus, ASXL1 appears to be involved in both PRC2-mediated gene repression and counteracting PRC1 ubiquitination (Scheuermann, de Ayala Alonso et al. 2010, Abdel-Wahab, Adli et al. 2012, Chung, Schatoff et al. 2012, Dey, Seshasayee et al. 2012, Abdel-Wahab and Dey 2013). Furthermore, it was shown that H2Bub1 plays a tumor suppressor function and also reported to be decreased during tumor progression in breast cancer (Shema, Tirosh et al. 2008, Prenzel, Begus-Nahrmann et al. 2011). To understand epigenetic roles of ASXL1, we examined whether depletion of ASXL1 altered the global histone methylation and monoubiquitination levels in MCF10A cells. Western blot analysis showed decreased levels of global H3K4me3 and H2Bub1 and increased levels of H2Aub1 and no effect on H3K27me3 levels in ASXL1 depleted cells compared with control cells. 
These results indicate that $A S X L 1$ is required to maintain $H 3$ methylation and $H 2 A$ monoubiquitination (Fig. 24).

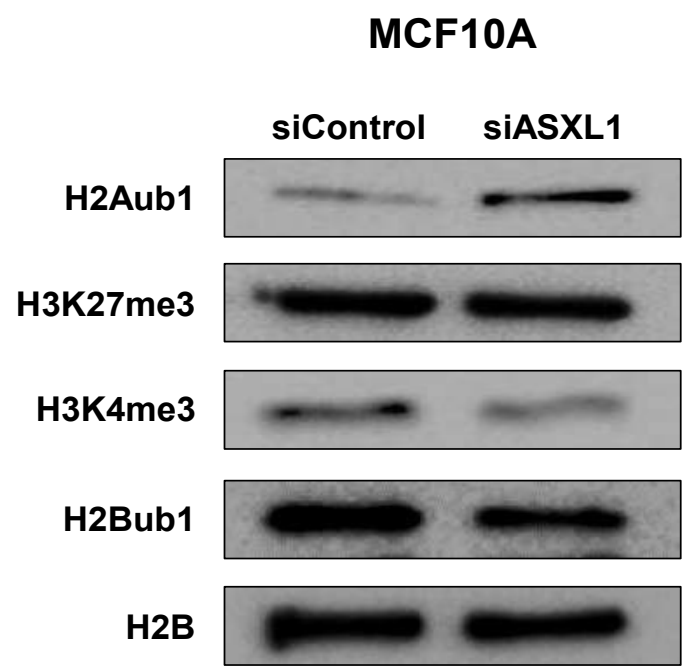

Figure. 24: ASXL1 depletion regulates histone methylation and monoubiquitination levels. ASXL1 depletion by siASXL1 decreases H2Bub1, H3K4me3 protein levels and increased H2AK119Ub protein levels. H2B was used as a loading control.

\subsubsection{ASXL1 enhances the gene expression by epigenetic regulation}

Methylation of lysine 4 on histone $\mathrm{H} 3(\mathrm{H} 3 \mathrm{~K} 4)$ at promoters is tightly linked to transcriptional regulation in human cells and also $\mathrm{H} 3 \mathrm{~K} 4 \mathrm{me} 3$ is known to be an important histone modification coupled with transcription activation (Okitsu, Hsieh et al. 2010, Dong, Tsuji et al. 2015). In order to investigate the mechanisms by which ASXL1 regulates PTEN, ARID1B and TJP3 expression, we checked the average H3K4me3 signals $( \pm 5 K b)$ around the TSS region of ASXL1-bound genes genomewide in an aggregate plot for all the ASXL1-bound genes. And, we observed H3K4me3 enrichment across the ASXL1 bound peaks. Published ChIP-seq data for H3K4me3 (SRR398029) (Choe, Hong et al. 2012) were used for the analysis (Fig. 25) 


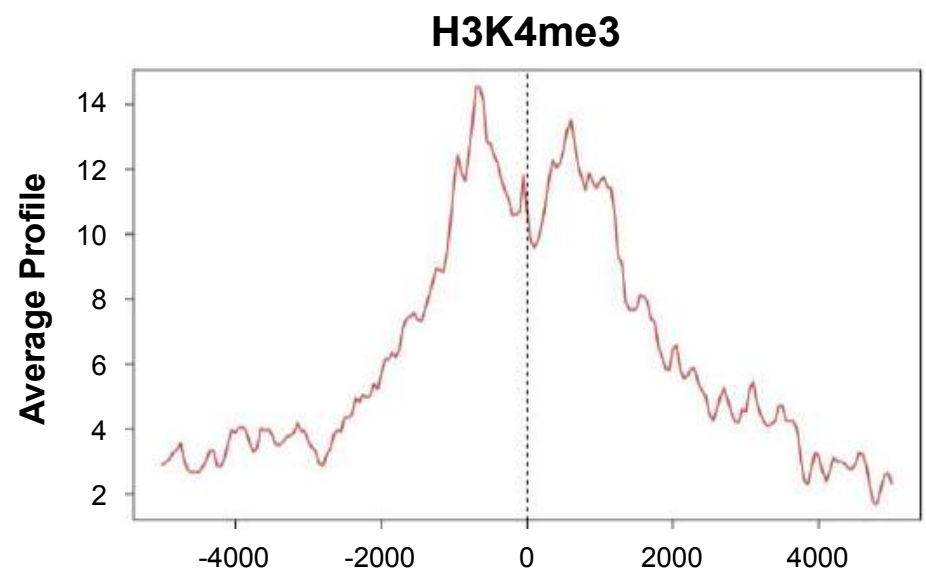

Relative Distance from the Centre (bp)

Figure. 25: H3K4me3 enrichment correlates with gene expression. Published H3K4me3 ChIP-seq was used for the aggregate plot analysis on the ASXL1-bound peaks genomewide. The analysis was performed around the $( \pm 5 \mathrm{~Kb})$ TSS of the genes.

\subsubsection{ASXL1 depletion leads to decreased H3K4me3 on ASXL1 bound genes}

To investigate the occupancy of H3K4me3 on ASXL1 bound genes, H3K4me3 ChIPseq data was used for the genome-wide and single gene analyses, which showed H3K4me3 enrichment around the transcription start site (TSS). Prominent peak was observed around the promoters of ASXL1 bound genes (PTEN and ARID1B) (Fig. 26A). To confirm the enrichment of H3K4me3 on ASXL1 bound genes, we performed H3K4me3 ChIP in MCF10A cells with or without ASXI1 depletion and also in MDA-MB-231 cells with stable ASXL1 expression. The enrichment of H3K4me3 was confirmed by ChIP-qRT-PCR. Consistent with the ChIP-seq results, qRT-PCR analysis of ChIP samples showed a significant enrichment of ASXL1 binding on the TSS region of ASXL1 bound (PTEN, TJP3 and ARID1B) genes. Furthermore, the qRT-PCR analysis showed a significant decrease of H3K4me3 enrichment on the TSS upon ASXL1 depletion whereas a significant increase of H3K4me3 enrichment on the TSS upon ASXI1 over-expression (Fig. 26B). Taken together, these results 
confirm that ASXL1 promotes increased H3K4me3 occupancy on the genes and subsequent gene activation.

A

H3K4me3

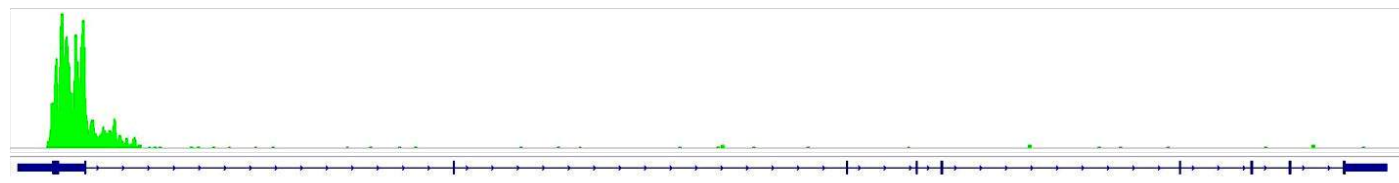

PTEN

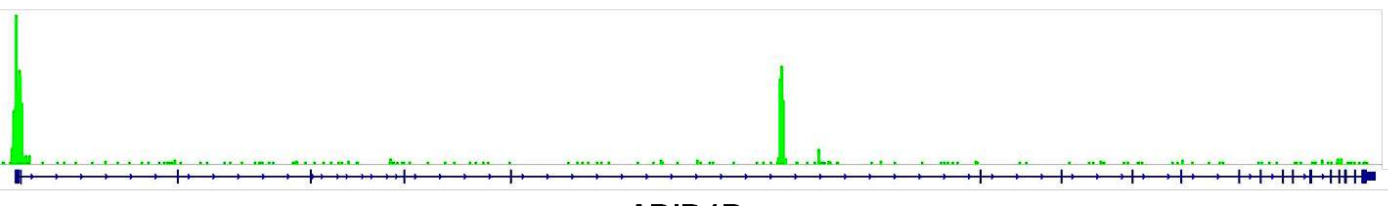

ARID1B 
B

\section{MCF10A}

PTEN TSS

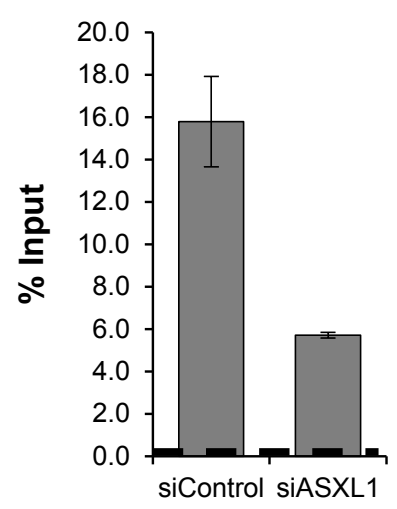

PTEN TSS

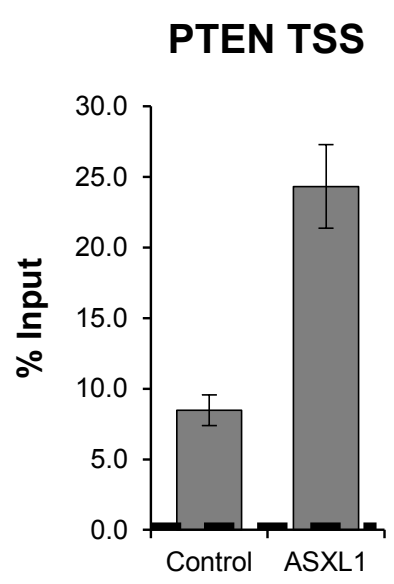

TJP3 TSS

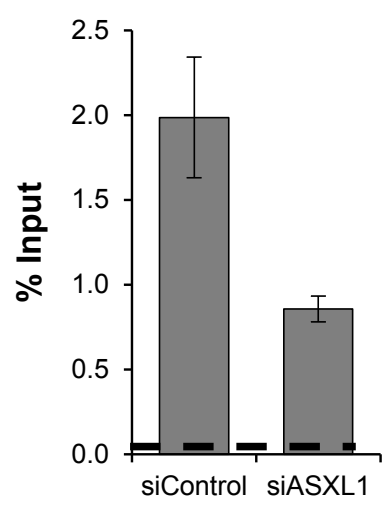

MDA-MB-231
TJP3 TSS

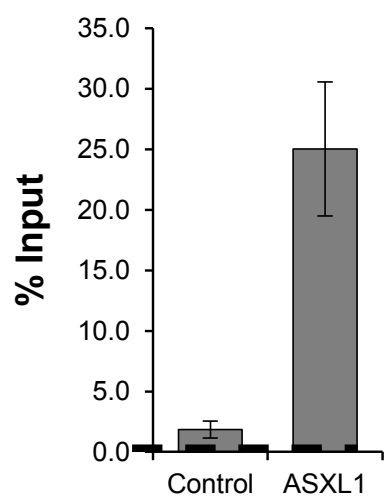

ARID1B TSS

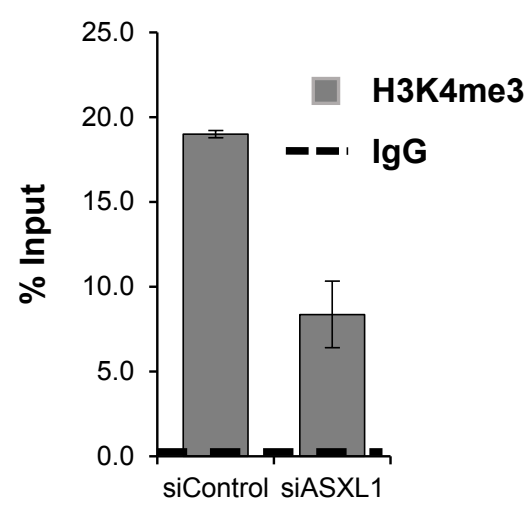

ARID1B TSS

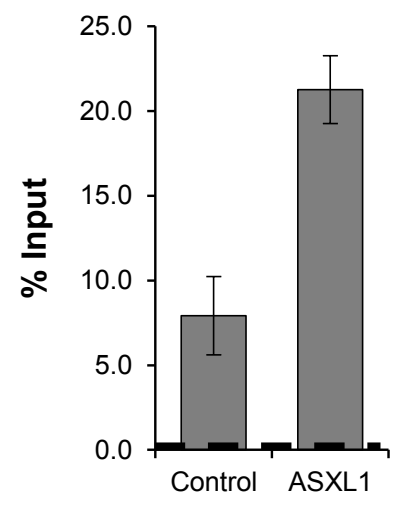

Figure. 26: H3K4me3 enrichment on the promoters of ASXL1 target genes. (A) ChIPseq profile of H3K4me3 on PTEN and ARID1B genes. Significant peak was observed around the TSS. (B) ChIP analysis of H3K4me3 enrichment on TSS of single genes in MCF10A cells with and without ASXL1 depletion and with and without ASXL1 overexpression in MDA-MB-231 cells. Immunoprecipitated DNA is compared to input and represented as "\% of Input". IgG antibody was used a negative control to measure the background signal level and displayed as a dotted line. Data are shown as mean $\pm S D$. $n=3$.

\subsubsection{ASXL1 cooperates with SET1 methylase complex to epigenetically enhance gene expression}

To further explain how ASXL1 positively regulates the H3K4me3 level at the promoters of the ASXL1 bound genes, we performed co-immunoprecipitation of 
ASXL1 to check the interaction with SET1 complex and BAP1. SET1 complex methylate the histone 3 lysine 4 (H3K4) (Thornton, Westfield et al. 2014). Recently, BAP1 was shown to interact with and deubiquitinate the transcriptional regulator host cell factor 1 (HCF-1) which is one of the members of SET1 complex (Lee, Tate et al. 2007, Narayanan, Ruyechan et al. 2007, Machida, Machida et al. 2009). We observed that ASXL1 physically interacts with H3K4-specific methyltransferase SET1 complex members (Cfp1 and Wdr82) and furthermore we also found that BAP1 also interacts with Wdr82 (Fig. 27)

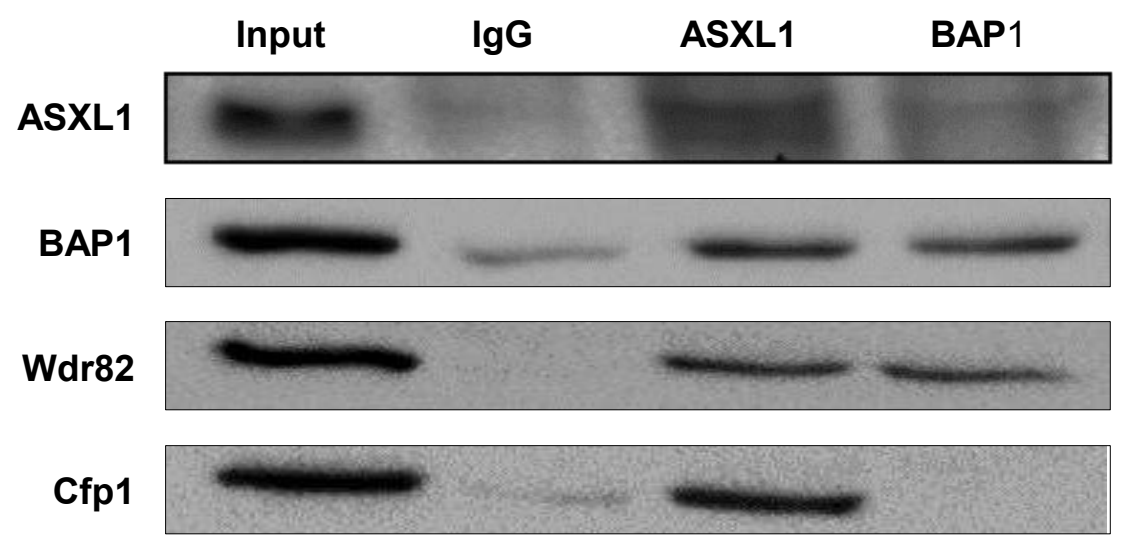

Figure. 27: ASXL1 and BAP1 associate with SET1 complex members. Immunoprecipitation followed by immunoblotting showed that ASXL1 interacts with Wdr82 and Cpf1 whereas BAP1 interacts with only Wdr82.

\subsubsection{Depletion of ASXL1 leads to decreased expression of SET1 complex members}

It was reported that the coactivator HCF-1 is required for recruitment of Setd1A and MLL1 and BAP1 is known to deubiquitinate HCF-1. From our Co-IP results, we found that ASXL1 and BAP1 were interacting with SET1 complex members. We performed immunoblotting to check the expression of Wdr82 and Cfp1 in ASXL1 depleted cells and also in ASXL1 overexpressing cells (Fig. 28). In ASXL1 depleted cells, we observed that Wdr82 expression was significantly decreased whereas Cfp1 
expression was partially decreased, which may affect the stability of SET1 complex and decreased methylation. In ASXL1 overexpressing cells there was increased Wdr82 expression whereas Cfp1 expression was not affected. Previously, it was shown that Wdr82 depletion leads to decreased Setd1A and Cfp1 expression (Lee and Skalnik 2008). Hence, Wdr82 is required for the targeting of Setd1A-mediated histone H3-Lys4 trimethylation near transcription start sites.

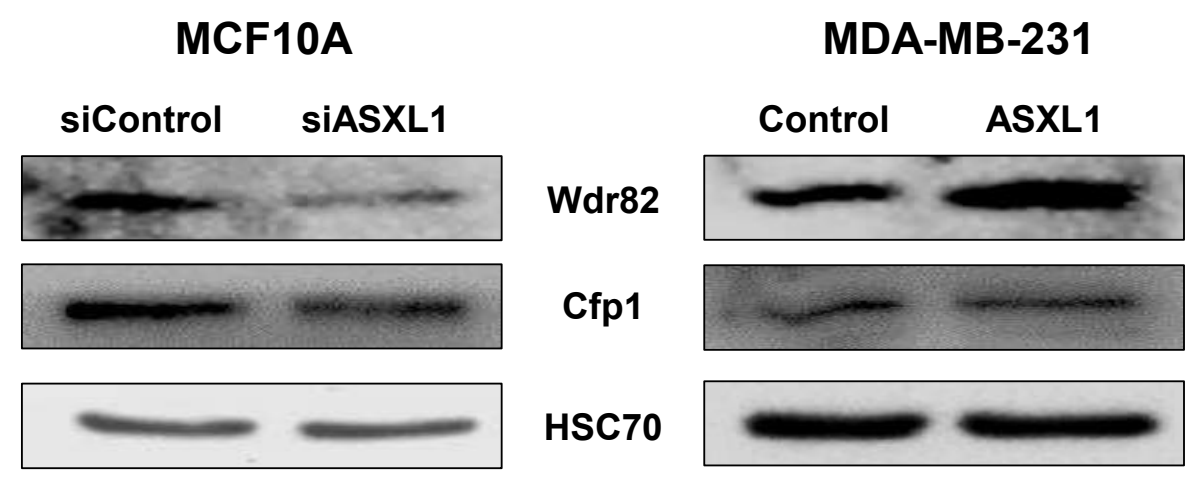

Figure. 28: ASXL1 depletion regulates expression of SET1 complex members. The expression of SET1 complex members, Cfp1 and Wdr82 was checked by immunoblotting. 


\section{Discussion}

Breast cancer is the most frequently diagnosed cancer and the leading cause of cancer death in females and most of them are caused due to tumor invasion and metastasis. Genetic and epigenetic alterations in the normal cells lead to the formation of the malignant cells. The malignant cells lose control over the cellular processes including cell morphology, proliferation, migration, cell death and eventually forms a tumor. The cellular plasticity, the fundamental characteristic required by the cells during embryo development, plays an essential role in cancer development and metastasis, in which the epithelial tumor cells undergo enormous cellular modifications and transdifferentiate into mesenchymal cells by activating the epithelial-to-mesenchymal transition (EMT) process (Boyer and Thiery 1993, Hay 1995, Vicente-Duenas, Gutierrez de Diego et al. 2009). In order to metastasize to distant sites, these cells reorganize their cytoskeleton, breakaway from tumor and migrate, invade and colonize to form secondary tumor by undergoing mesenchymalto-epithelial transition (MET) (Chambers, Groom et al. 2002, Bacac and Stamenkovic 2008, Tsai and Yang 2013). Epigenetic processes play a vital role in the normal development and tissue-specific gene expression and its maintenance. However, the aberrant epigenetic state leads to an altered gene expression which can transform a normal cell into the malignant cell (Sharma, Kelly et al. 2010, Tam and Weinberg 2013). ASXL1 is one among the frequently mutated genes in malignant myeloid diseases (Gelsi-Boyer, Brecqueville et al. 2012). ASXL1 was reported to be one of the components of various chromatin modifying complexes which regulate gene expression. (Abdel-Wahab, Adli et al. 2012). In this study, we focused on characterizing the role of ASXL1 in breast cancer with an aim to reveal the 
mechanism involved in ASXL1 mediated tumorigenesis and metastasis and to establish new therapeutic targets for preventing cancer progression and metastasis in various cancers.

\subsection{The role of ASXL1 as a potential tumor suppressor}

The development of cancer is a multistep and complex process and is initiated by the inactivation of tumor suppressor genes or the activation of oncogenes, which can eliminate the control on cell growth and proliferation and eventually transform a normal cell into a cancerous cell (Hahn and Weinberg 2002). Tumor suppressor genes function by keeping a constant check on cell proliferation and inducing apoptosis to maintain the tissue homeostasis, which is a native suppression of tumor formation. So, inactivation or loss of tumor suppressors results in loss of control over cell growth which is considered as one of the most important hallmarks of cancer development (Hanahan and Weinberg 2011). Based on the context-dependent functions of ASXL family members as transcription activators or repressors, these proteins are proposed to be tumor suppressive or oncogenic, which depends on the genes they activate or repress (Katoh 2013). ASXL1 is mutated in several types of cancers such as lung squamous and bladder cancer (Futreal, Coin et al. 2004, Kandoth, McLellan et al. 2013) ASXL1 is frequently mutated in myeloid malignancies and hematological cancers. ASXL1 mutations are associated with disease progression and poor clinical outcome (Abdel-Wahab, Adli et al. 2012, Valletta, Dolatshad et al. 2015, Wu, Bekker-Jensen et al. 2015). ASXL1 mutations are strongly associated with poorer survival in patients with myeloid malignancies (Shih, Abdel-Wahab et al. 2012). 
In our study, the examination of ASXL1 mRNA expression status in a wide spectrum of breast carcinoma datasets revealed the significant downregulation of ASXL1 mRNA expression levels in breast cancer samples compared to the control samples. We have also tested the clinical utility of ASXL1 protein expression as a prognostic biomarker of breast cancer progression through examination of the association between ASXL1 and disease outcome. In our analysis, we found that the patients with low ASXL1 expression have significantly poorer relapse-free survival than the patients with high ASXL1 expression and collectively, there is an inverse correlation of ASXL1 expression with tumor malignancy. However, in-depth studies are needed to understand the role of ASXL1 in tumorigenesis completely.

\subsection{ASXL1 downregulation perturbs the global gene expression}

Depletion or inactivation of tumor suppressor genes leads to the reduced function of the gene. Loss of function leads to the increase in tumor cell number by increasing cell growth or by inhibiting cell death, which is caused due to the activation of genes regulating cell cycle or by suppressing the genes involved in apoptosis (Vogelstein and Kinzler 2004). As an initial step to understand the functional role of ASXL1 in breast cancer development and progression, the transcriptional effect of ASXL1 depletion was obtained through mRNA expression profiling in normal breast epithelial cells, MCF10A. The transcriptome-wide sequencing analysis revealed that the expression of ASXL1 activates or suppresses a distinct set of genes which includes a small portion of genes involved in EMT process such as fibronectin, Vimentin, TJP-3, N-cadherin and E-cadherin and also stem cell marker, CD24. Other than EMT genes, a small subset of genes is also differentially expressed. PTEN, a tumor suppressor is one among them which is known to be mutated or lost in several 
cancers, including breast cancer and involved in tumorigenesis and progression (Zhang, Liang et al. 2013). ASXL1 depletion resulted in the perturbation of genes that are involved in cell adhesion, cell proliferation and cell motility. The putative tumor suppressor role of ASXL1 was confirmed by the GSEA and the DAVID functional annotation analyses. The gene Ontology analysis reveals that ASXL1 controls cellular activities, that are exploited during cancer progression and metastasis, such as cell adhesion, cell proliferation, angiogenesis, cell death and cell motility. Furthermore, gene expression analyses of ASXL1 depleted MCF10A cells confirms that pathways related to breast cancer, epithelial differentiation, EMT and metastasis are among the top enriched pathways. This suggests that ASXL1 depletion promotes EMT.

\subsection{The role of ASXL1 in cancer metastasis}

EMT and MET processes play an important role in the transition between epithelial and mesenchymal phenotype and are involved in the normal development and cancer metastasis. During the transition, the epithelial cells disrupt their intracellular tight junctions and cell-cell contacts and become mesenchymal cells. These cells acquire stem cell properties and enhance their invasive and migratory properties (Thiery and Sleeman 2006, Heerboth, Housman et al. 2015). Our analysis of global gene expression suggested the role of ASXL1 in EMT regulation and thus metastasis. To assess more and test further consequences, ASXL1 is either depleted or overexpressed.

\subsubsection{The role of ASXL1 in regulating EMT}

One of the important clues that have been obtained in this study is the possible involvement of ASXL1 in the EMT process. EMT is known to be indispensable for the 
development of the embryo and the formation of various tissues or organs. However, inappropriate activation of EMT in the normal cells leads to diseases, such as cancer by converting the epithelial cancer cells into stem-cell like mesenchymal cancer cells. In normal epithelial cells, the genes which make the cells in association with other cells in a tissue are expressed, however, the mesenchymal genes, which induce the cells to change morphology, and to breakaway for migration and invasion, are repressed. E-cadherin is involved in cell-cell adhesion and its loss is thought to be the initial step for enabling EMT and metastasis. In contrast, $\mathrm{N}$-cadherin is elevated in EMT and can regulate cell-cell adhesion during the invasion. The switch of E-cadherin and $\mathrm{N}$-cadherin expression induces EMT in several cancers, leading to enhanced cellular motility or invasion and decreased adhesion (Mani, Guo et al. 2008, Onder, Gupta et al. 2008, Gupta, Onder et al. 2009).

Our study reveals that depletion of ASXL1 induces a dramatic change in the morphology of MCF10A cells from epithelial to fibroblastic-like spindle-shaped phenotype. The alteration in cell morphology is one of the characteristics of EMT and is associated with changes in the expression of several genes. In our transcriptomewide analysis, we found that ASXL1 depletion significantly upregulates or downregulates some of the EMT-associated genes, which includes a reduced expression of epithelial markers such as E-cadherin and TJP3 and an increased expression of mesenchymal markers such as Vimentin, N-cadherin and fibronectin.

To further determine the tumor suppressor role of ASXL1 in breast cancer, we evaluated the expression of ASXL1 in breast cancer cell line, MDA-MB-231 and observed that ASXL1 expression was downregulated. Furthermore, stable ASXL1 overexpression in both normal and breast cancer cells exhibited increased epithelial 
marker expression and decreased mesenchymal marker expression. The epithelial marker, E-cadherin was decreased whereas the mesenchymal markers, Vimentin, and N-cadherin were increased in ASXL1 depleted cells. In contrast, there is an increase in E-cadherin expression and a decrease in $\mathrm{N}$-cadherin and Vimentin expression in the cells with ASXL1 overexpression. Immunofluorescent staining confirmed increased expression of Vimentin and reduced expression of E-cadherin in ASXL1 depleted MCF10A cells compared to the control cells, suggesting that these factors might contribute to induction of the EMT phenotype. The migration and invasion of cancer cells is an important process of cancer metastasis. Our studies using in vitro migration assays showed an increased migration potential of the normal mammary epithelial cell lines MCF10A and MCF12A upon ASXL1 depletion, whereas ASXL1 overexpression suppressed the migratory potential of the cells (MCF10A). More importantly, ASXL1 overexpression inhibited the migration of breast cancer cells (MDA-MB-231). Enhanced cell migration is a pronounced feature of advanced tumors (Friedl, Hegerfeldt et al. 2004) and therefore decreased ASXL1 expression may potentially facilitate cancer progression.

Furthermore, due to the inverse relationship between the expression of epithelial genes and mesenchymal genes, it seems that ASXL1, with its tumor suppressor function, may suppress the mesenchymal genes. Indeed, our data highlights the same that depletion of ASXL1 results in the upregulation of mesenchymal markers whereas overexpression results in repression of mesenchymal markers. Taken together, we have shown in this study that, upon depletion of ASXL1, mammary epithelial cells undergo a series of changes through which cells lose their epithelial characteristics and acquire the mesenchymal phenotype. 


\subsubsection{ASXL1 expression regulates cancer stem cell pool}

Several reports have shown that breast cancer stem cells (BCSCs) have been implicated as the main driver for initiation, maintenance, progression and reoccurrence of breast cancer. Cancer stem cells (CSCs) are a small portion of undifferentiated malignant cancer cells within a tumor that have the capacity to selfrenew, proliferate and can differentiate/transdifferentiate into the heterogeneous population of cancer cells. (Clarke, Dick et al. 2006, Yao, Ping et al. 2011, Li, Yao et al. 2014, Liu and Fan 2015, Qiao, Liang et al. 2015). Recently, it was reported that EMT has been closely linked to the acquisition of CSCs-like characteristics such as enhanced self-renewal, CSC-gene expression and increased tumorigenicity (Mani, Guo et al. 2008, Sampieri and Fodde 2012). In our studies, we observed that the CSC marker, CD24 was downregulated in ASXL1 depleted mammary cells at both mRNA and protein level. Furthermore, ASXL1 depletion also increased the mammosphere formation which was suppressed by the overexpression of ASXL1 in MCF10A cells. Taken together, the results strongly suggest the role of ASXL1 in mammospheres formation and anchorage-independent growth by suppressing the stem cell phenotype.

\subsection{Genome-wide distribution of ASXL1}

From our transcriptome-wide data, we found the potential role of ASXL1 in regulating EMT. In order to gain mechanistic insight into the function of ASXL1 in EMT regulation, we need to understand ASXL1 mediated regulation of gene transcription. From our genome-wide analyses, we observed that the occupancy of ASXL1 was mostly confined to the distal intergenic regions. However, a significant portion of ASXL1 binding sites was confined to the promoter regions throughout the genome. 
Furthermore, aggregate plot analyses also revealed that the highest enrichment of ASXL1 was observed near the TSS region of the genes. Possible mechanistic explanations for the presumed tumor-suppressor activity of ASXL1 can be studied by identifying the ASXL1 regulated genes. In our IGV data significant ASXL1 peaks were found around the promoter regions of a small subset of ASXL1 regulated genes such as PTEN, TJP3 and ARID1B. Furthermore, the qRT-PCR analysis showed that there was a significant decrease of ASXL1 binding on the TSS of PTEN, ARID1B and TJP3 upon ASXL1 knockdown. Moreover, there was a significant increase in ASXL1 occupancy upon ASXL1 overexpression which proves the specificity of ASXL1.

A key issue that remains to be elucidated is whether ASXL1 binding has a direct function in gene transcription. Indeed, we observed that these genes were significantly downregulated upon ASXL1 depletion and upregulated upon ASXL1 overexpression. These results demonstrate that ASXL1 regulates gene transcription.

\subsection{ASXL1 regulates EMT by targeting PTEN}

PTEN, a tumor suppressor gene, is a negative regulator of (PI3K)-Akt pathway and participates in the regulation of cell cycle, proliferation, apoptosis, cell adhesion and EMT during embryonic development and cancer progression. PTEN is known to dephosphorylate Akt and inactivate PI3K/Akt pathway and its depletion leads to accumulation of an active form of Akt (Cantley and Neel 1999, Nicholson, Streuli et al. 2003). PTEN mutations were reported in several cancers and its loss leads to induction of tumor invasion and metastasis (Wang, Quah et al. 2007, Keniry and Parsons 2008, Bao, Yan et al. 2013). In our ChIP-seq analysis, we found that ASXL1 occupancy on the PTEN promoter and PTEN expression was regulated 
depending upon ASXL1 expression. Thus, loss of PTEN perturbs the EMT genes. As a result of depletion of PTEN in MCF10A and MDA-MB-231 cells, the expression of epithelial markers, CDH1 and TJP3 were downregulated and mesenchymal markers, $\mathrm{CDH} 2$, VIM and FN1 were upregulated without any effect on the expression of ASXL1. Thus, this indicates that PTEN may be one of the main downstream regulators of ASXL1. However, the ectopic expression of ASXL1 was able to suppress the expression of mesenchymal markers and retain the expression of epithelial markers. Furthermore, p-Akt was increased upon ASXL1 depletion whereas decreased upon ASXL1 overexpression. As PTEN is a downstream regulator of $A S X L 1$, knockdown of PTEN in ASXL1 overexpressing MDA-MB-231 cells rescued the phosphorylation of Akt. Thus, our results suggest that ASXL1 inhibits Akt signaling through positive regulation of PTEN expression.

\subsection{ASXL1 and post-translational modifications}

It was reported that $A S X L 1$ regulates epigenetic marks and transcription through interaction with polycomb complex proteins, thus ASXL1 loss results in the reduction of K3K27me3, the repressive mark regulated by the PRC2 complex (Cho, Kim et al. 2006, Boultwood, Perry et al. 2010, Wang, Li et al. 2014). It was also reported that ASXL1 depletion disrupts the PR-DUB complex which in turn results in an increase in H2Aub1 levels. In our study, we show that depletion of ASXL1 is associated with global changes in histone modifications such as decreased levels of global H3K4me3 levels and increased levels of global H2Aub1 levels. We also observed that there is a slight reduction of H2Bub1 levels. However, H3K27me3 levels were not affected. Global loss of H2Bub1 has been reported in breast cancer (Prenzel, Begus-Nahrmann et al. 2011) and H2Bub1 is known to recruit the methyltransferase 
complex, which is involved in H3K4 methylation (Wood, Schneider et al. 2005, Kim, Guermah et al. 2009). H3K4me3 is associated with transcription activation and enrichment of ASXL1 at promoter regions genome-wide suggesting the potential role of that $A S X L 1$ in the regulation of gene transcription.

\subsection{Loss of ASXL1 is associated with occupancy of $\mathrm{H} 3 \mathrm{~K} 4 \mathrm{me} 3$}

As it was observed in this study, depletion of ASXL1 leads to a reduction in global H3K4me3 levels. Therefore, the H3K4me3 binding on various direct ASXL1 target genes (PTEN, TJP3, and ARID1B) following ASXL1 knockdown and overexpression was analyzed. Our ChIP-qRT-PCR in ASXL1 depleted cells revealed a significant reduction of $\mathrm{H} 3 \mathrm{~K} 4 \mathrm{me} 3$ enrichment on $\mathrm{ASXL1}$ target genes whereas ASXL1 overexpression showed increased occupancy of H3K4me3. Furthermore, aggregate plot analyses of publicly available ChIP-seq data for H3K4me3 showed that the highest enrichment of H3K4me3 was observed near the TSS region of the ASXL1 bound genes. Our data suggests that ASXL1 target genes expression was altered through direct binding and also by altering the chromatin state of promoters.

\subsection{ASXL1 physically interacts with BAP1 and SET1 methyltransferase complex members}

Recent studies have identified several interacting partners for ASXL family members, such as BAP1, PRC2 complex members (EZH2 and EED) and nuclear receptors (Cho, Kim et al. 2006, Scheuermann, de Ayala Alonso et al. 2010, Park, Yoon et al. 2011, Lai and Wang 2013). In this study, we showed that ASXL1 depletion leads to the reduction in both global and promoter-associated $\mathrm{H} 3 \mathrm{~K} 4 \mathrm{me} 3$. Our results clearly demonstrate that ASXL1 interacts with BAP1, Cfp1 and Wdr82, whereas BAP1 interacts with ASXL1 and Wdr82. BAP1 is the catalytic subunit of PR-DUB, which 
deubiquitinates the H2Aub1. Furthermore, our studies demonstrated for the first time that ASXL1 physically interact with SET1 complex members. Taken together, our results indicate that ASXL1 may form a complex with SET1 complex members and co-regulate target gene expression in part through $\mathrm{H} 3 \mathrm{~K} 4 \mathrm{me} 3$.

\subsection{ASXL1 regulates expression of SET1 complex members}

It was reported that BAP1 not only interacts with HCF1 but also deubiquitinates HCF1, which is a member of SET1 complex and is involved in the recruitment of Setd1A and MLL1, which leads to transcription activation through H3K4me3 (Lee, Tate et al. 2007, Narayanan, Ruyechan et al. 2007, Tyagi, Chabes et al. 2007, Machida, Machida et al. 2009). Our results show that SET1 complex members were interacting with ASXL1 and BAP1. Interestingly, we also observed the expression of Wdr82 was significantly decreased whereas Cfp1 expression was partially decreased upon ASXL1 depletion. Furthermore, we also observed increased expression of Wdr82, but there was no change in Cfp1 expression upon ASXL1 overexpression. It was reported that depletion of Wdr82 leads to decreased expression of Setd1A, which in turn leads to the decreased H3K4me3 at the TSS as a result of decreased occupancy of Setd1A. It was also reported that depletion of Wdr82 downregulates Set1A expression and effects the stability of SET1 complex (Lee and Skalnik 2008, Wu, Wang et al. 2008). Our data suggests that ASXL1 mediates the expression of SET1 complex members and regulates H3K4me3 through SET1 complex. 


\section{Conclusion and Future Perspectives}

Altogether, in this study, we have elucidated the tumor suppressor role of ASXL1 in breast cancer, which inhibits EMT and regulates stem cell properties in mammary cells. RNA-seq analysis revealed that the loss of ASXL1 results in upregulation of EMT gene signatures and also induces migratory properties. Furthermore, based on the genome-wide and transcriptome-wide analysis, we found PTEN as a direct target gene of ASXL1 and depletion of PTEN leads to EMT phenotype. Interestingly, we also found that ASXL1 interacts with a methyltransferase, SET1 complex members and their expression were also regulated by ASXL1. More studies are required to further validate the mechanism and regulatory function of ASXL1 that is coupled with transcription and EMT. Furthermore, ChIP analysis or ChIP-seq against SET1 complex members to determine genome-wide co-occupancy of ASXL1 and SET1 complex members on ASXL1 target genes to promote transcription activation. 


\section{References}

Abdel-Wahab, O., M. Adli, L. M. LaFave, J. Gao, T. Hricik, A. H. Shih, S. Pandey, J. P. Patel, Y. R. Chung, R. Koche, F. Perna, X. Zhao, J. E. Taylor, C. Y. Park, M. Carroll, A. Melnick, S. D. Nimer, J. D. Jaffe, I. Aifantis, B. E. Bernstein and R. L. Levine (2012). "ASXL1 mutations promote myeloid transformation through loss of PRC2-mediated gene repression." Cancer Cell 22(2): 180-193.

Abdel-Wahab, O. and A. Dey (2013). "The ASXL-BAP1 axis: new factors in myelopoiesis, cancer and epigenetics." Leukemia 27(1): 10-15.

Abdel-Wahab, O., J. Gao, M. Adli, A. Dey, T. Trimarchi, Y. R. Chung, C. Kuscu, T. Hricik, D. Ndiaye-Lobry, L. M. Lafave, R. Koche, A. H. Shih, O. A. Guryanova, E. Kim, S. Li, S. Pandey, J. Y. Shin, L. Telis, J. Liu, P. K. Bhatt, S. Monette, X. Zhao, C. E. Mason, C. Y. Park, B. E. Bernstein, I. Aifantis and R. L. Levine (2013). "Deletion of Asxl1 results in myelodysplasia and severe developmental defects in vivo." J Exp Med 210(12): 26412659.

Al-Hajj, M., M. S. Wicha, A. Benito-Hernandez, S. J. Morrison and M. F. Clarke (2003). "Prospective identification of tumorigenic breast cancer cells." Proc Natl Acad Sci U S A 100(7): 3983-3988.

Albert, M. and K. Helin (2010). "Histone methyltransferases in cancer." Semin Cell Dev Biol 21(2): 209-220.

Allan, J., G. J. Cowling, N. Harborne, P. Cattini, R. Craigie and H. Gould (1981). "Regulation of the higher-order structure of chromatin by histones $\mathrm{H} 1$ and $\mathrm{H} 5$." $\underline{\mathrm{J} \text { Cell Biol }}$ 90(2): 279-288.

Amente, S., L. Lania and B. Majello (2013). "The histone LSD1 demethylase in stemness and cancer transcription programs." Biochim Biophys Acta 1829(10): 981-986.

Anastas, J. N. and R. T. Moon (2013). "WNT signalling pathways as therapeutic targets in cancer." Nat Rev Cancer 13(1): 11-26.

Anders, S., P. T. Pyl and W. Huber (2015). "HTSeq--a Python framework to work with highthroughput sequencing data." Bioinformatics 31(2): 166-169.

Aumann, S. and O. Abdel-Wahab (2014). "Somatic alterations and dysregulation of epigenetic modifiers in cancers." Biochem Biophys Res Commun 455(1-2): 24-34.

Bacac, M. and I. Stamenkovic (2008). "Metastatic cancer cell." Annu Rev Pathol 3: 221-247.

Bainbridge, M. N., H. Hu, D. M. Muzny, L. Musante, J. R. Lupski, B. H. Graham, W. Chen, K. W. Gripp, K. Jenny, T. F. Wienker, Y. Yang, V. R. Sutton, R. A. Gibbs and H. H. Ropers (2013). "De novo truncating mutations in ASXL3 are associated with a novel clinical phenotype with similarities to Bohring-Opitz syndrome." Genome Med 5(2): 11.

Balasubramani, A., A. Larjo, J. A. Bassein, X. Chang, R. B. Hastie, S. M. Togher, H. Lahdesmaki and A. Rao (2015). "Cancer-associated ASXL1 mutations may act as gainof-function mutations of the ASXL1-BAP1 complex." Nat Commun 6: 7307. 
Bannister, A. J., R. Schneider and T. Kouzarides (2002). "Histone methylation: dynamic or static?" Cell 109(7): 801-806.

Bao, L., Y. Yan, C. Xu, W. Ji, S. Shen, G. Xu, Y. Zeng, B. Sun, H. Qian, L. Chen, M. Wu, C. Su and J. Chen (2013). "MicroRNA-21 suppresses PTEN and hSulf-1 expression and promotes hepatocellular carcinoma progression through AKT/ERK pathways." Cancer Lett 337(2): 226-236.

Batlle, E., E. Sancho, C. Franci, D. Dominguez, M. Monfar, J. Baulida and A. Garcia De Herreros (2000). "The transcription factor snail is a repressor of E-cadherin gene expression in epithelial tumour cells." Nat Cell Biol 2(2): 84-89.

Bedford, M. T. and S. G. Clarke (2009). "Protein arginine methylation in mammals: who, what, and why." Mol Cell 33(1): 1-13.

Beisel, C. and R. Paro (2011). "Silencing chromatin: comparing modes and mechanisms." Nat Rev Genet 12(2): 123-135.

Bellacosa, A., D. de Feo, A. K. Godwin, D. W. Bell, J. Q. Cheng, D. A. Altomare, M. Wan, L. Dubeau, G. Scambia, V. Masciullo, G. Ferrandina, P. Benedetti Panici, S. Mancuso, G. Neri and J. R. Testa (1995). "Molecular alterations of the AKT2 oncogene in ovarian and breast carcinomas." Int J Cancer 64(4): 280-285.

Bhanot, P., M. Brink, C. H. Samos, J. C. Hsieh, Y. Wang, J. P. Macke, D. Andrew, J. Nathans and R. Nusse (1996). "A new member of the frizzled family from Drosophila functions as a Wingless receptor." Nature 382(6588): 225-230.

Bird, A. (2002). "DNA methylation patterns and epigenetic memory." Genes Dev 16(1): 6-21.

Boultwood, J., J. Perry, A. Pellagatti, M. Fernandez-Mercado, C. Fernandez-Santamaria, M. J. Calasanz, M. J. Larrayoz, M. Garcia-Delgado, A. Giagounidis, L. Malcovati, M. G. Della Porta, M. Jadersten, S. Killick, E. Hellstrom-Lindberg, M. Cazzola and J. S. Wainscoat (2010). "Frequent mutation of the polycomb-associated gene ASXL1 in the myelodysplastic syndromes and in acute myeloid leukemia." Leukemia 24(5): 10621065.

Bowen, K. A., H. Q. Doan, B. P. Zhou, Q. Wang, Y. Zhou, P. G. Rychahou and B. M. Evers (2009). "PTEN loss induces epithelial--mesenchymal transition in human colon cancer cells." Anticancer Res 29(11): 4439-4449.

Boyer, B. and J. P. Thiery (1993). "Epithelium-mesenchyme interconversion as example of epithelial plasticity." APMIS 101(4): 257-268.

Brock, H. W. and M. van Lohuizen (2001). "The Polycomb group--no longer an exclusive club?" Curr Opin Genet Dev 11(2): 175-181.

Burgoyne, L. A., J. D. Mobbs and A. J. Marshall (1976). "Chromatin structure: a property of the higher structures of chromatin and in the time course of its formation during chromatin replication." Nucleic Acids Res 3(12): 3293-3304.

Buus, R., M. Faronato, D. E. Hammond, S. Urbe and M. J. Clague (2009). "Deubiquitinase activities required for hepatocyte growth factor-induced scattering of epithelial cells." Curr Biol 19(17): 1463-1466. 
Byles, V., L. Zhu, J. D. Lovaas, L. K. Chmilewski, J. Wang, D. V. Faller and Y. Dai (2012). "SIRT1 induces EMT by cooperating with EMT transcription factors and enhances prostate cancer cell migration and metastasis." Oncogene 31(43): 4619-4629.

Cadigan, K. M. and R. Nusse (1997). "Wnt signaling: a common theme in animal development." Genes Dev 11(24): 3286-3305.

Caja, L., E. Bertran, J. Campbell, N. Fausto and I. Fabregat (2011). "The transforming growth factor-beta (TGF-beta) mediates acquisition of a mesenchymal stem cell-like phenotype in human liver cells." J Cell Physiol 226(5): 1214-1223.

Caldeira, J. R., E. C. Prando, F. C. Quevedo, F. A. Neto, C. A. Rainho and S. R. Rogatto (2006). "CDH1 promoter hypermethylation and E-cadherin protein expression in infiltrating breast cancer." BMC Cancer 6: 48.

Campos, E. I. and D. Reinberg (2009). "Histones: annotating chromatin." Annu Rev Genet 43: 559-599.

Cano, A., M. A. Perez-Moreno, I. Rodrigo, A. Locascio, M. J. Blanco, M. G. del Barrio, F. Portillo and M. A. Nieto (2000). "The transcription factor snail controls epithelialmesenchymal transitions by repressing E-cadherin expression." Nat Cell Biol 2(2): 7683.

Cantley, L. C. and B. G. Neel (1999). "New insights into tumor suppression: PTEN suppresses tumor formation by restraining the phosphoinositide 3-kinase/AKT pathway." Proc Natl Acad Sci U S A 96(8): 4240-4245.

Cao, R., Y. Tsukada and Y. Zhang (2005). "Role of Bmi-1 and Ring1A in H2A ubiquitylation and Hox gene silencing." Mol Cell 20(6): 845-854.

Carbuccia, N., A. Murati, V. Trouplin, M. Brecqueville, J. Adelaide, J. Rey, W. Vainchenker, O. A. Bernard, M. Chaffanet, N. Vey, D. Birnbaum and M. J. Mozziconacci (2009). "Mutations of ASXL1 gene in myeloproliferative neoplasms." Leukemia 23(11): 21832186.

Cedar, H. and Y. Bergman (2009). "Linking DNA methylation and histone modification: patterns and paradigms." Nat Rev Genet 10(5): 295-304.

Chambers, A. F., A. C. Groom and I. C. MacDonald (2002). "Dissemination and growth of cancer cells in metastatic sites." Nat Rev Cancer 2(8): 563-572.

Chang, B., Y. Chen, Y. Zhao and R. K. Bruick (2007). "JMJD6 is a histone arginine demethylase." Science 318(5849): 444-447.

Chen, L., T. Kasai, Y. Li, Y. Sugii, G. Jin, M. Okada, A. Vaidyanath, A. Mizutani, A. Satoh, T. Kudoh, M. J. Hendrix, D. S. Salomon, L. Fu and M. Seno (2012). "A model of cancer stem cells derived from mouse induced pluripotent stem cells." PLoS One 7(4): e33544.

Chen, T., K. Yang, J. Yu, W. Meng, D. Yuan, F. Bi, F. Liu, J. Liu, B. Dai, X. Chen, F. Wang, F. Zeng, H. Xu, J. Hu and X. Mo (2012). "Identification and expansion of cancer stem cells in tumor tissues and peripheral blood derived from gastric adenocarcinoma patients." Cell Res 22(1): 248-258. 
Cheng, J. Q., A. K. Godwin, A. Bellacosa, T. Taguchi, T. F. Franke, T. C. Hamilton, P. N. Tsichlis and J. R. Testa (1992). "AKT2, a putative oncogene encoding a member of a subfamily of protein-serine/threonine kinases, is amplified in human ovarian carcinomas." Proc Natl Acad Sci U S A 89(19): 9267-9271.

Chi, P., C. D. Allis and G. G. Wang (2010). "Covalent histone modifications--miswritten, misinterpreted and mis-erased in human cancers." Nat Rev Cancer 10(7): 457-469.

Cho, Y. S., E. J. Kim, U. H. Park, H. S. Sin and S. J. Um (2006). "Additional sex comb-like 1 (ASXL1), in cooperation with SRC-1, acts as a ligand-dependent coactivator for retinoic acid receptor." J Biol Chem 281(26): 17588-17598.

Choe, M. K., C. P. Hong, J. Park, S. H. Seo and T. Y. Roh (2012). "Functional elements demarcated by histone modifications in breast cancer cells." Biochem Biophys Res Commun 418(3): 475-482.

Choudhary, C., C. Kumar, F. Gnad, M. L. Nielsen, M. Rehman, T. C. Walther, J. V. Olsen and M. Mann (2009). "Lysine acetylation targets protein complexes and co-regulates major cellular functions." Science 325(5942): 834-840.

Chung, Y. R., E. Schatoff and O. Abdel-Wahab (2012). "Epigenetic alterations in hematopoietic malignancies." Int J Hematol 96(4): 413-427.

Clarke, M. F., J. E. Dick, P. B. Dirks, C. J. Eaves, C. H. Jamieson, D. L. Jones, J. Visvader, I. L. Weissman and G. M. Wahl (2006). "Cancer stem cells--perspectives on current status and future directions: AACR Workshop on cancer stem cells." $\underline{\text { Cancer Res }}$ 66(19): 9339-9344.

Comijn, J., G. Berx, P. Vermassen, K. Verschueren, L. van Grunsven, E. Bruyneel, M. Mareel, D. Huylebroeck and F. van Roy (2001). "The two-handed E box binding zinc finger protein SIP1 downregulates E-cadherin and induces invasion." Mol Cell 7(6): 1267-1278.

Das, C., M. S. Lucia, K. C. Hansen and J. K. Tyler (2009). "CBP/p300-mediated acetylation of histone H3 on lysine 56." Nature 459(7243): 113-117.

De Craene, B. and G. Berx (2013). "Regulatory networks defining EMT during cancer initiation and progression." Nat Rev Cancer 13(2): 97-110.

de la Cruz, X., S. Lois, S. Sanchez-Molina and M. A. Martinez-Balbas (2005). "Do protein motifs read the histone code?" Bioessays 27(2): 164-175.

Derynck, R. and R. J. Akhurst (2007). "Differentiation plasticity regulated by TGF-beta family proteins in development and disease." Nat Cell Biol 9(9): 1000-1004.

Dey, A., D. Seshasayee, R. Noubade, D. M. French, J. Liu, M. S. Chaurushiya, D. S. Kirkpatrick, V. C. Pham, J. R. Lill, C. E. Bakalarski, J. Wu, L. Phu, P. Katavolos, L. M. LaFave, O. Abdel-Wahab, Z. Modrusan, S. Seshagiri, K. Dong, Z. Lin, M. Balazs, R. Suriben, K. Newton, S. Hymowitz, G. Garcia-Manero, F. Martin, R. L. Levine and V. M. Dixit (2012). "Loss of the tumor suppressor BAP1 causes myeloid transformation." Science 337(6101): 1541-1546.

Di Cerbo, V. and R. Schneider (2013). "Cancers with wrong HATs: the impact of acetylation." Brief Funct Genomics 12(3): 231-243. 
Dietrich, B. H., J. Moore, M. Kyba, G. dosSantos, F. McCloskey, T. A. Milne, H. W. Brock and $\mathrm{H}$. M. Krause (2001). "Tantalus, a novel ASX-interacting protein with tissue-specific functions." Dev Biol 234(2): 441-453.

Dong, C., Y. Wu, J. Yao, Y. Wang, Y. Yu, P. G. Rychahou, B. M. Evers and B. P. Zhou (2012). "G9a interacts with Snail and is critical for Snail-mediated E-cadherin repression in human breast cancer." J Clin Invest 122(4): 1469-1486.

Dong, P., Y. Konno, H. Watari, M. Hosaka, M. Noguchi and N. Sakuragi (2014). "The impact of microRNA-mediated PI3K/AKT signaling on epithelial-mesenchymal transition and cancer stemness in endometrial cancer." J Transl Med 12: 231.

Dong, X., J. Tsuji, A. Labadorf, P. Roussos, J. F. Chen, R. H. Myers, S. Akbarian and Z. Weng (2015). "The Role of H3K4me3 in Transcriptional Regulation Is Altered in Huntington's Disease." PLoS One 10(12): e0144398.

Dragu, D. L., L. G. Necula, C. Bleotu, C. C. Diaconu and M. Chivu-Economescu (2015). "Therapies targeting cancer stem cells: Current trends and future challenges." World J Stem Cells 7(9): 1185-1201.

Dutnall, R. N. and V. Ramakrishnan (1997). "Twists and turns of the nucleosome: tails without ends." Structure 5(10): 1255-1259.

Easwaran, V., M. Pishvaian, Salimuddin and S. Byers (1999). "Cross-regulation of betacatenin-LEF/TCF and retinoid signaling pathways." Curr Biol 9(23): 1415-1418.

Eccles, S. A. and D. R. Welch (2007). "Metastasis: recent discoveries and novel treatment strategies." Lancet 369(9574): 1742-1757.

Eger, A., K. Aigner, S. Sonderegger, B. Dampier, S. Oehler, M. Schreiber, G. Berx, A. Cano, $H$. Beug and R. Foisner (2005). "DeltaEF1 is a transcriptional repressor of E-cadherin and regulates epithelial plasticity in breast cancer cells." Oncogene 24(14): 2375-2385.

Egger, G., G. Liang, A. Aparicio and P. A. Jones (2004). "Epigenetics in human disease and prospects for epigenetic therapy." Nature 429(6990): 457-463.

Enkhbaatar, Z., M. Terashima, D. Oktyabri, S. Tange, A. Ishimura, S. Yano and T. Suzuki (2013). "KDM5B histone demethylase controls epithelial-mesenchymal transition of cancer cells by regulating the expression of the microRNA-200 family." Cell Cycle 12(13): 2100-2112.

Esquela-Kerscher, A. and F. J. Slack (2006). "Oncomirs - microRNAs with a role in cancer." Nat Rev Cancer 6(4): 259-269.

Feinberg, A. P., R. Ohlsson and S. Henikoff (2006). "The epigenetic progenitor origin of human cancer." Nat Rev Genet 7(1): 21-33.

Felsenfeld, G. and M. Groudine (2003). "Controlling the double helix." Nature 421(6921): 448-453.

Fernando, J., A. Malfettone, E. B. Cepeda, R. Vilarrasa-Blasi, E. Bertran, G. Raimondi, A. Fabra, A. Alvarez-Barrientos, P. Fernandez-Salguero, C. M. Fernandez-Rodriguez, G. Giannelli, P. Sancho and I. Fabregat (2015). "A mesenchymal-like phenotype and 
expression of CD44 predict lack of apoptotic response to sorafenib in liver tumor cells." Int J Cancer 136(4): E161-172.

Ferrari-Amorotti, G., V. Fragliasso, R. Esteki, Z. Prudente, A. R. Soliera, S. Cattelani, G. Manzotti, G. Grisendi, M. Dominici, M. Pieraccioli, G. Raschella, C. Chiodoni, M. P. Colombo and B. Calabretta (2013). "Inhibiting interactions of lysine demethylase LSD1 with snail/slug blocks cancer cell invasion." Cancer Res 73(1): 235-245.

Firestein, R., A. J. Bass, S. Y. Kim, I. F. Dunn, S. J. Silver, I. Guney, E. Freed, A. H. Ligon, N. Vena, S. Ogino, M. G. Chheda, P. Tamayo, S. Finn, Y. Shrestha, J. S. Boehm, S. Jain, E. Bojarski, C. Mermel, J. Barretina, J. A. Chan, J. Baselga, J. Tabernero, D. E. Root, C. S. Fuchs, M. Loda, R. A. Shivdasani, M. Meyerson and W. C. Hahn (2008). "CDK8 is a colorectal cancer oncogene that regulates beta-catenin activity." Nature 455(7212): 547-551.

Fischle, W., Y. Wang and C. D. Allis (2003). "Histone and chromatin cross-talk." Curr Opin Cell Biol 15(2): 172-183.

Fisher, C. L., J. Berger, F. Randazzo and H. W. Brock (2003). "A human homolog of Additional sex combs, ADDITIONAL SEX COMBS-LIKE 1, maps to chromosome 20q11." Gene 306: 115-126.

Fisher, C. L., I. Lee, S. Bloyer, S. Bozza, J. Chevalier, A. Dahl, C. Bodner, C. D. Helgason, J. L. Hess, R. K. Humphries and H. W. Brock (2010). "Additional sex combs-like 1 belongs to the enhancer of trithorax and polycomb group and genetically interacts with Cbx2 in mice." Dev Biol 337(1): 9-15.

Fisher, C. L., N. Pineault, C. Brookes, C. D. Helgason, H. Ohta, C. Bodner, J. L. Hess, R. K. Humphries and H. W. Brock (2010). "Loss-of-function Additional sex combs like 1 mutations disrupt hematopoiesis but do not cause severe myelodysplasia or leukemia." Blood 115(1): 38-46.

Fisher, C. L., F. Randazzo, R. K. Humphries and H. W. Brock (2006). "Characterization of Asxl1, a murine homolog of Additional sex combs, and analysis of the Asx-like gene family." Gene 369: 109-118.

Fletcher, T. M. and J. C. Hansen (1996). "The nucleosomal array: structure/function relationships." Crit Rev Eukaryot Gene Expr 6(2-3): 149-188.

Fournier, M. V., J. E. Fata, K. J. Martin, P. Yaswen and M. J. Bissell (2009). "Interaction of E-cadherin and PTEN regulates morphogenesis and growth arrest in human mammary epithelial cells." Cancer Res 69(10): 4545-4552.

Fraga, M. F. and M. Esteller (2005). "Towards the human cancer epigenome: a first draft of histone modifications." Cell Cycle 4(10): 1377-1381.

Fraile, J. M., V. Quesada, D. Rodriguez, J. M. Freije and C. Lopez-Otin (2012). "Deubiquitinases in cancer: new functions and therapeutic options." Oncogene 31(19): 2373-2388.

Franke, W. W., C. Grund, C. Kuhn, B. W. Jackson and K. Illmensee (1982). "Formation of cytoskeletal elements during mouse embryogenesis. III. Primary mesenchymal cells and the first appearance of vimentin filaments." Differentiation 23(1): 43-59. 
Friedl, P., Y. Hegerfeldt and M. Tusch (2004). "Collective cell migration in morphogenesis and cancer." Int J Dev Biol 48(5-6): 441-449.

Futreal, P. A., L. Coin, M. Marshall, T. Down, T. Hubbard, R. Wooster, N. Rahman and M. R. Stratton (2004). "A census of human cancer genes." Nat Rev Cancer 4(3): 177-183.

Fuxe, J., T. Vincent and A. Garcia de Herreros (2010). "Transcriptional crosstalk between TGF-beta and stem cell pathways in tumor cell invasion: role of EMT promoting Smad complexes." Cell Cycle 9(12): 2363-2374.

Gallahan, D. and R. Callahan (1997). "The mouse mammary tumor associated gene INT3 is a unique member of the NOTCH gene family (NOTCH4)." Oncogene 14(16): 18831890.

Gelsi-Boyer, V., M. Brecqueville, R. Devillier, A. Murati, M. J. Mozziconacci and D. Birnbaum (2012). "Mutations in ASXL1 are associated with poor prognosis across the spectrum of malignant myeloid diseases." $\mathrm{J}$ Hematol Oncol 5: 12.

Gelsi-Boyer, V., V. Trouplin, J. Adelaide, J. Bonansea, N. Cervera, N. Carbuccia, A. Lagarde, T. Prebet, M. Nezri, D. Sainty, S. Olschwang, L. Xerri, M. Chaffanet, M. J. Mozziconacci, N. Vey and D. Birnbaum (2009). "Mutations of polycomb-associated gene ASXL1 in myelodysplastic syndromes and chronic myelomonocytic leukaemia." $\mathrm{Br}$ J Haematol 145(6): 788-800.

Gigi, O., B. Geiger, Z. Eshhar, R. Moll, E. Schmid, S. Winter, D. L. Schiller and W. W. Franke (1982). "Detection of a cytokeratin determinant common to diverse epithelial cells by a broadly cross-reacting monoclonal antibody." EMBO J 1(11): 1429-1437.

Gildea, J. J., R. Lopez and A. Shearn (2000). "A screen for new trithorax group genes identified little imaginal discs, the Drosophila melanogaster homologue of human retinoblastoma binding protein 2." Genetics 156(2): 645-663.

Giroldi, L. A., P. P. Bringuier, M. de Weijert, C. Jansen, A. van Bokhoven and J. A. Schalken (1997). "Role of $E$ boxes in the repression of E-cadherin expression." Biochem Biophys Res Commun 241(2): 453-458.

Glinsky, G. V., O. Berezovska and A. B. Glinskii (2005). "Microarray analysis identifies a death-from-cancer signature predicting therapy failure in patients with multiple types of cancer." J Clin Invest 115(6): 1503-1521.

Gomis, R. R., C. Alarcon, C. Nadal, C. Van Poznak and J. Massague (2006). "C/EBPbeta at the core of the TGFbeta cytostatic response and its evasion in metastatic breast cancer cells." Cancer Cell 10(3): 203-214.

Gonzalo, S. (2010). "Epigenetic alterations in aging." J Appl Physiol (1985) 109(2): 586-597.

Grabher, C., H. von Boehmer and A. T. Look (2006). "Notch 1 activation in the molecular pathogenesis of T-cell acute lymphoblastic leukaemia." Nat Rev Cancer 6(5): 347-359.

Grasso, C. S., Y. M. Wu, D. R. Robinson, X. Cao, S. M. Dhanasekaran, A. P. Khan, M. J. Quist, X. Jing, R. J. Lonigro, J. C. Brenner, I. A. Asangani, B. Ateeq, S. Y. Chun, J. Siddiqui, L. Sam, M. Anstett, R. Mehra, J. R. Prensner, N. Palanisamy, G. A. Ryslik, F. Vandin, B. J. Raphael, L. P. Kunju, D. R. Rhodes, K. J. Pienta, A. M. Chinnaiyan and S. 
A. Tomlins (2012). "The mutational landscape of lethal castration-resistant prostate cancer." Nature 487(7406): 239-243.

Grewal, S. I. and S. C. Elgin (2007). "Transcription and RNA interference in the formation of heterochromatin." Nature 447(7143): 399-406.

Grille, S. J., A. Bellacosa, J. Upson, A. J. Klein-Szanto, F. van Roy, W. Lee-Kwon, M. Donowitz, P. N. Tsichlis and L. Larue (2003). "The protein kinase Akt induces epithelial mesenchymal transition and promotes enhanced motility and invasiveness of squamous cell carcinoma lines." Cancer Res 63(9): 2172-2178.

Grimaud, C., N. Negre and G. Cavalli (2006). "From genetics to epigenetics: the tale of Polycomb group and trithorax group genes." Chromosome Res 14(4): 363-375.

Grimshaw, M. J., L. Cooper, K. Papazisis, J. A. Coleman, H. R. Bohnenkamp, L. ChiaperoStanke, J. Taylor-Papadimitriou and J. M. Burchell (2008). "Mammosphere culture of metastatic breast cancer cells enriches for tumorigenic breast cancer cells." Breast Cancer Res 10(3): R52.

Grunstein, M. (1997). "Histone acetylation in chromatin structure and transcription." Nature 389(6649): 349-352.

Gu, G., J. Yuan, M. Wills and S. Kasper (2007). "Prostate cancer cells with stem cell characteristics reconstitute the original human tumor in vivo." Cancer Res 67(10): 48074815.

Guo, X. and X. F. Wang (2009). "Signaling cross-talk between TGF-beta/BMP and other pathways." Cell Res 19(1): 71-88.

Gupta, G. P. and J. Massague (2006). "Cancer metastasis: building a framework." Cell 127(4): 679-695.

Gupta, P. B., T. T. Onder, G. Jiang, K. Tao, C. Kuperwasser, R. A. Weinberg and E. S. Lander (2009). "Identification of selective inhibitors of cancer stem cells by highthroughput screening." Cell 138(4): 645-659.

Hahn, W. C. and R. A. Weinberg (2002). "Modelling the molecular circuitry of cancer." Nat Rev Cancer 2(5): 331-341.

Hajra, K. M., D. Y. Chen and E. R. Fearon (2002). "The SLUG zinc-finger protein represses E-cadherin in breast cancer." Cancer Res 62(6): 1613-1618.

Halachmi, N., K. L. Schulze, A. Inbal and A. Salzberg (2007). "Additional sex combs affects antennal development by means of spatially restricted repression of Antp and wg." Dev Dyn 236(8): 2118-2130.

Hanahan, D. and R. A. Weinberg (2011). "Hallmarks of cancer: the next generation." Cell 144(5): 646-674.

Hargreaves, D. C. and G. R. Crabtree (2011). "ATP-dependent chromatin remodeling: genetics, genomics and mechanisms." Cell Res 21(3): 396-420.

Hay, E. D. (1995). "An overview of epithelio-mesenchymal transformation." Acta Anat (Basel) 154(1): 8-20. 
He, X., J. P. Saint-Jeannet, Y. Wang, J. Nathans, I. Dawid and H. Varmus (1997). "A member of the Frizzled protein family mediating axis induction by Wnt-5A." Science 275(5306): 1652-1654.

Heerboth, S., G. Housman, M. Leary, M. Longacre, S. Byler, K. Lapinska, A. Willbanks and S. Sarkar (2015). "EMT and tumor metastasis." Clin Transl Med 4: 6.

Heldin, C. H., M. Vanlandewijck and A. Moustakas (2012). "Regulation of EMT by TGFbeta in cancer." FEBS Lett 586(14): 1959-1970.

Henikoff, S. (2000). "Heterochromatin function in complex genomes." Biochim Biophys Acta 1470(1): 01-8.

Hennig, G., J. Behrens, M. Truss, S. Frisch, E. Reichmann and W. Birchmeier (1995). "Progression of carcinoma cells is associated with alterations in chromatin structure and factor binding at the E-cadherin promoter in vivo." Oncogene 11(3): 475-484.

Herranz, N., D. Pasini, V. M. Diaz, C. Franci, A. Gutierrez, N. Dave, M. Escriva, I. Hernandez-Munoz, L. Di Croce, K. Helin, A. Garcia de Herreros and S. Peiro (2008). "Polycomb complex 2 is required for E-cadherin repression by the Snail 1 transcription factor." Mol Cell Biol 28(15): 4772-4781.

Hershko, A. and A. Ciechanover (1998). "The ubiquitin system." Annu Rev Biochem 67: 425479.

Horn, P. J. and C. L. Peterson (2002). "Molecular biology. Chromatin higher order folding-wrapping up transcription." Science 297(5588): 1824-1827.

Huang, J., T. Fan, Q. Yan, H. Zhu, S. Fox, H. J. Issaq, L. Best, L. Gangi, D. Munroe and K. Muegge (2004). "Lsh, an epigenetic guardian of repetitive elements." Nucleic Acids Res 32(17): 5019-5028.

Huang, J., L. Zhang, J. Greshock, T. A. Colligon, Y. Wang, R. Ward, D. Katsaros, H. Lassus, R. Butzow, A. K. Godwin, J. R. Testa, K. L. Nathanson, P. A. Gimotty, G. Coukos, B. L. Weber and Y. Degenhardt (2011). "Frequent genetic abnormalities of the PI3K/AKT pathway in primary ovarian cancer predict patient outcome." Genes Chromosomes Cancer 50(8): 606-618.

Huang, R. Y., P. Guilford and J. P. Thiery (2012). "Early events in cell adhesion and polarity during epithelial-mesenchymal transition." J Cell Sci 125(Pt 19): 4417-4422.

Imhof, A., X. J. Yang, V. V. Ogryzko, Y. Nakatani, A. P. Wolffe and H. Ge (1997). "Acetylation of general transcription factors by histone acetyltransferases." Curr Biol 7(9): 689-692.

Inoue, Y., Y. Itoh, K. Sato, F. Kawasaki, C. Sumita, T. Tanaka, D. Morishita and H. Hayashi (2016). "Regulation of Epithelial-Mesenchymal Transition by E3 Ubiquitin Ligases and Deubiquitinase in Cancer." Curr Cancer Drug Targets 16(2): 110-118.

Jaggupilli, A. and E. Elkord (2012). "Significance of CD44 and CD24 as cancer stem cell markers: an enduring ambiguity." Clin Dev Immunol 2012: 708036.

Jemal, A., F. Bray, M. M. Center, J. Ferlay, E. Ward and D. Forman (2011). "Global cancer statistics." CA Cancer J Clin 61(2): 69-90. 
Jenuwein, T. and C. D. Allis (2001). "Translating the histone code." Science 293(5532): 1074-1080.

Johnsen, S. A. (2012). "The enigmatic role of H2Bub1 in cancer." FEBS Lett 586(11): 15921601.

Jones, P. A. and S. B. Baylin (2007). "The epigenomics of cancer." Cell 128(4): 683-692.

Jones, P. A. and P. W. Laird (1999). "Cancer epigenetics comes of age." Nat Genet 21(2): 163-167.

Jones, S., X. Zhang, D. W. Parsons, J. C. Lin, R. J. Leary, P. Angenendt, P. Mankoo, H. Carter, H. Kamiyama, A. Jimeno, S. M. Hong, B. Fu, M. T. Lin, E. S. Calhoun, M. Kamiyama, K. Walter, T. Nikolskaya, Y. Nikolsky, J. Hartigan, D. R. Smith, M. Hidalgo, S. D. Leach, A. P. Klein, E. M. Jaffee, M. Goggins, A. Maitra, C. lacobuzio-Donahue, J. R. Eshleman, S. E. Kern, R. H. Hruban, R. Karchin, N. Papadopoulos, G. Parmigiani, B. Vogelstein, V. E. Velculescu and K. W. Kinzler (2008). "Core signaling pathways in human pancreatic cancers revealed by global genomic analyses." Science 321(5897): 1801-1806.

Kanamori, Y., J. Kigawa, H. Itamochi, M. Shimada, M. Takahashi, S. Kamazawa, S. Sato, R. Akeshima and N. Terakawa (2001). "Correlation between loss of PTEN expression and Akt phosphorylation in endometrial carcinoma." Clin Cancer Res 7(4): 892-895.

Kandoth, C., M. D. McLellan, F. Vandin, K. Ye, B. Niu, C. Lu, M. Xie, Q. Zhang, J. F. McMichael, M. A. Wyczalkowski, M. D. Leiserson, C. A. Miller, J. S. Welch, M. J. Walter, M. C. Wendl, T. J. Ley, R. K. Wilson, B. J. Raphael and L. Ding (2013). "Mutational landscape and significance across 12 major cancer types." Nature 502(7471): 333-339.

Kang, Y. and J. Massague (2004). "Epithelial-mesenchymal transitions: twist in development and metastasis." Cell 118(3): 277-279.

Kangaspeska, S., B. Stride, R. Metivier, M. Polycarpou-Schwarz, D. Ibberson, R. P. Carmouche, V. Benes, F. Gannon and G. Reid (2008). "Transient cyclical methylation of promoter DNA." Nature 452(7183): 112-115.

Karpiuk, O., Z. Najafova, F. Kramer, M. Hennion, C. Galonska, A. Konig, N. Snaidero, T. Vogel, A. Shchebet, Y. Begus-Nahrmann, M. Kassem, M. Simons, H. Shcherbata, T. Beissbarth and S. A. Johnsen (2012). "The histone H2B monoubiquitination regulatory pathway is required for differentiation of multipotent stem cells." Mol Cell 46(5): 705713.

Katoh, M. (2013). "Functional and cancer genomics of ASXL family members." $\mathrm{Br} \mathrm{J}$ Cancer 109(2): 299-306.

Katoh, M. and M. Katoh (2003). "Identification and characterization of ASXL2 gene in silico." Int J Oncol 23(3): 845-850.

Katoh, M. and M. Katoh (2004). "Identification and characterization of ASXL3 gene in silico." Int J Oncol 24(6): 1617-1622.

Katsuno, Y., S. Lamouille and R. Derynck (2013). "TGF-beta signaling and epithelialmesenchymal transition in cancer progression." Curr Opin Oncol 25(1): 76-84. 
Keil, K. P., L. L. Abler, V. Mehta, H. M. Altmann, J. Laporta, E. H. Plisch, M. Suresh, L. L. Hernandez and C. M. Vezina (2014). "DNA methylation of E-cadherin is a priming mechanism for prostate development." Dev Biol 387(2): 142-153.

Keniry, M. and R. Parsons (2008). "The role of PTEN signaling perturbations in cancer and in targeted therapy." Oncogene 27(41): 5477-5485.

Kim, D., G. Pertea, C. Trapnell, H. Pimentel, R. Kelley and S. L. Salzberg (2013). "TopHat2: accurate alignment of transcriptomes in the presence of insertions, deletions and gene fusions." Genome Biol 14(4): R36.

Kim, H. J., B. C. Litzenburger, X. Cui, D. A. Delgado, B. C. Grabiner, X. Lin, M. T. Lewis, M. M. Gottardis, T. W. Wong, R. M. Attar, J. M. Carboni and A. V. Lee (2007). "Constitutively active type I insulin-like growth factor receptor causes transformation and xenograft growth of immortalized mammary epithelial cells and is accompanied by an epithelial-to-mesenchymal transition mediated by NF-kappaB and snail." Mol Cell Biol 27(8): 3165-3175.

Kim, J., M. Guermah, R. K. McGinty, J. S. Lee, Z. Tang, T. A. Milne, A. Shilatifard, T. W. Muir and R. G. Roeder (2009). "RAD6-Mediated transcription-coupled H2B ubiquitylation directly stimulates H3K4 methylation in human cells." Cell 137(3): 459471.

Kim, Y. M. and M. Kahn (2014). "The role of the Wnt signaling pathway in cancer stem cells: prospects for drug development." Res Rep Biochem 4: 1-12.

Komatsu, H., M. Y. Chao, J. Larkins-Ford, M. E. Corkins, G. A. Somers, T. Tucey, H. M. Dionne, J. Q. White, K. Wani, M. Boxem and A. C. Hart (2008). "OSM-11 facilitates LIN12 Notch signaling during Caenorhabditis elegans vulval development." PLoS Biol 6(8): e196.

Kopper, L. and M. Hajdu (2004). "Tumor stem cells." Pathol Oncol Res 10(2): 69-73.

Kornberg, R. D. (1974). "Chromatin structure: a repeating unit of histones and DNA." Science 184(4139): 868-871.

Kouzarides, T. (2007). "SnapShot: Histone-modifying enzymes." Cell 131(4): 822.

Laemmli, U. K. (1970). "Cleavage of structural proteins during the assembly of the head of bacteriophage T4." Nature 227(5259): 680-685.

LaFave, L. M., W. Beguelin, R. Koche, M. Teater, B. Spitzer, A. Chramiec, E. Papalexi, M. D. Keller, T. Hricik, K. Konstantinoff, J. B. Micol, B. Durham, S. K. Knutson, J. E. Campbell, G. Blum, X. Shi, E. H. Doud, A. V. Krivtsov, Y. R. Chung, I. Khodos, E. de Stanchina, O. Ouerfelli, P. S. Adusumilli, P. M. Thomas, N. L. Kelleher, M. Luo, H. Keilhack, O. Abdel-Wahab, A. Melnick, S. A. Armstrong and R. L. Levine (2015). "Loss of BAP1 function leads to EZH2-dependent transformation." Nat Med 21(11): 13441349.

Lai, H. L. and Q. T. Wang (2013). "Additional sex combs-like 2 is required for polycomb repressive complex 2 binding at select targets." PLoS One 8(9): e73983.

Lamouille, S., J. Xu and R. Derynck (2014). "Molecular mechanisms of epithelialmesenchymal transition." Nat Rev Mol Cell Biol 15(3): 178-196. 
Langmead, B. and S. L. Salzberg (2012). "Fast gapped-read alignment with Bowtie 2." Nat Methods 9(4): 357-359.

Lapidot, T., C. Sirard, J. Vormoor, B. Murdoch, T. Hoang, J. Caceres-Cortes, M. Minden, B. Paterson, M. A. Caligiuri and J. E. Dick (1994). "A cell initiating human acute myeloid leukaemia after transplantation into SCID mice." Nature 367(6464): 645-648.

Latham, J. A. and S. Y. Dent (2007). "Cross-regulation of histone modifications." Nat Struct Mol Biol 14(11): 1017-1024.

Lee, J. H. and D. G. Skalnik (2008). "Wdr82 is a C-terminal domain-binding protein that recruits the Setd1A Histone H3-Lys4 methyltransferase complex to transcription start sites of transcribed human genes." Mol Cell Biol 28(2): 609-618.

Lee, J. H., C. M. Tate, J. S. You and D. G. Skalnik (2007). "Identification and characterization of the human Set1B histone H3-Lys4 methyltransferase complex." $\underline{\mathrm{J}}$ Biol Chem 282(18): 13419-13428.

Lee, S. W., Y. S. Cho, J. M. Na, U. H. Park, M. Kang, E. J. Kim and S. J. Um (2010). "ASXL1 represses retinoic acid receptor-mediated transcription through associating with HP1 and LSD1." J Biol Chem 285(1): 18-29.

Lei, W., K. Zhang, X. Pan, Y. Hu, D. Wang, X. Yuan, G. Shu and J. Song (2010). "Histone deacetylase 1 is required for transforming growth factor-beta1-induced epithelialmesenchymal transition." Int J Biochem Cell Biol 42(9): 1489-1497.

Li, B., Y. W. Zheng, Y. Sano and H. Taniguchi (2011). "Evidence for mesenchymal-epithelial transition associated with mouse hepatic stem cell differentiation." PLoS One 6(2): e17092.

Li, H., B. Handsaker, A. Wysoker, T. Fennell, J. Ruan, N. Homer, G. Marth, G. Abecasis, R. Durbin and S. Genome Project Data Processing (2009). "The Sequence Alignment/Map format and SAMtools." Bioinformatics 25(16): 2078-2079.

Li, M., H. Zhao, X. Zhang, L. D. Wood, R. A. Anders, M. A. Choti, T. M. Pawlik, H. D. Daniel, R. Kannangai, G. J. Offerhaus, V. E. Velculescu, L. Wang, S. Zhou, B. Vogelstein, R. H. Hruban, N. Papadopoulos, J. Cai, M. S. Torbenson and K. W. Kinzler (2011). "Inactivating mutations of the chromatin remodeling gene ARID2 in hepatocellular carcinoma." Nat Genet 43(9): 828-829.

Li, Q., Y. Yao, G. Eades, Z. Liu, Y. Zhang and Q. Zhou (2014). "Downregulation of miR-140 promotes cancer stem cell formation in basal-like early stage breast cancer." Oncogene 33(20): 2589-2600.

$\mathrm{Li}, \mathrm{Y}$. and H. Li (2012). "Many keys to push: diversifying the 'readership' of plant homeodomain fingers." Acta Biochim Biophys Sin (Shanghai) 44(1): 28-39.

Lin, T., A. Ponn, X. Hu, B. K. Law and J. Lu (2010). "Requirement of the histone demethylase LSD1 in Snai1-mediated transcriptional repression during epithelialmesenchymal transition." Oncogene 29(35): 4896-4904.

Liu, L., S. Qin, J. Zhang, P. Ji, Y. Shi and J. Wu (2012). "Solution structure of an atypical PHD finger in BRPF2 and its interaction with DNA." J Struct Biol 180(1): 165-173. 
Liu, X. and D. Fan (2015). "The epithelial-mesenchymal transition and cancer stem cells: functional and mechanistic links." Curr Pharm Des 21(10): 1279-1291.

Liu, Y. L., Y. M. Yang, H. Xu and X. S. Dong (2011). "Aberrant expression of USP22 is associated with liver metastasis and poor prognosis of colorectal cancer." J Surg Oncol 103(3): 283-289.

Logan, C. Y. and R. Nusse (2004). "The Wnt signaling pathway in development and disease." Annu Rev Cell Dev Biol 20: 781-810.

Love, M. I., W. Huber and S. Anders (2014). "Moderated estimation of fold change and dispersion for RNA-seq data with DESeq2." Genome Biol 15(12): 550.

Loyola, A. and G. Almouzni (2004). "Histone chaperones, a supporting role in the limelight." Biochim Biophys Acta 1677(1-3): 3-11.

Luger, K., A. W. Mader, R. K. Richmond, D. F. Sargent and T. J. Richmond (1997). "Crystal structure of the nucleosome core particle at 2.8 A resolution." Nature 389(6648): 251260.

Machida, Y. J., Y. Machida, A. A. Vashisht, J. A. Wohlschlegel and A. Dutta (2009). "The deubiquitinating enzyme BAP1 regulates cell growth via interaction with HCF-1." $\underline{\mathrm{J} \text { Biol }}$ Chem 284(49): 34179-34188.

Mani, S. A., W. Guo, M. J. Liao, E. N. Eaton, A. Ayyanan, A. Y. Zhou, M. Brooks, F. Reinhard, C. C. Zhang, M. Shipitsin, L. L. Campbell, K. Polyak, C. Brisken, J. Yang and R. A. Weinberg (2008). "The epithelial-mesenchymal transition generates cells with properties of stem cells." Cell 133(4): 704-715.

Marsden, M. P. and U. K. Laemmli (1979). "Metaphase chromosome structure: evidence for a radial loop model." Cell 17(4): 849-858.

Martin, F. T., R. M. Dwyer, J. Kelly, S. Khan, J. M. Murphy, C. Curran, N. Miller, E. Hennessy, P. Dockery, F. P. Barry, T. O'Brien and M. J. Kerin (2010). "Potential role of mesenchymal stem cells (MSCs) in the breast tumour microenvironment: stimulation of epithelial to mesenchymal transition (EMT)." Breast Cancer Res Treat 124(2): 317-326.

Maschler, S., G. Wirl, H. Spring, D. V. Bredow, I. Sordat, H. Beug and E. Reichmann (2005). "Tumor cell invasiveness correlates with changes in integrin expression and localization." Oncogene 24(12): 2032-2041.

Massague, J. (2000). "How cells read TGF-beta signals." Nat Rev Mol Cell Biol 1(3): 169178.

Mayer, I. A. and C. L. Arteaga (2016). "The PI3K/AKT Pathway as a Target for Cancer Treatment." Annu Rev Med 67: 11-28.

Metivier, R., R. Gallais, C. Tiffoche, C. Le Peron, R. Z. Jurkowska, R. P. Carmouche, D. Ibberson, P. Barath, F. Demay, G. Reid, V. Benes, A. Jeltsch, F. Gannon and G. Salbert (2008). "Cyclical DNA methylation of a transcriptionally active promoter." Nature 452(7183): 45-50. 
Micalizzi, D. S., S. M. Farabaugh and H. L. Ford (2010). "Epithelial-mesenchymal transition in cancer: parallels between normal development and tumor progression." $\underline{\mathrm{J} \text { Mammary }}$ Gland Biol Neoplasia 15(2): 117-134.

Miele, L. (2006). "Notch signaling." Clin Cancer Res 12(4): 1074-1079.

Miele, L., H. Miao and B. J. Nickoloff (2006). "NOTCH signaling as a novel cancer therapeutic target." Curr Cancer Drug Targets 6(4): 313-323.

Miele, L. and B. Osborne (1999). "Arbiter of differentiation and death: Notch signaling meets apoptosis." J Cell Physiol 181(3): 393-409.

Miller, T., N. J. Krogan, J. Dover, H. Erdjument-Bromage, P. Tempst, M. Johnston, J. F. Greenblatt and A. Shilatifard (2001). "COMPASS: a complex of proteins associated with a trithorax-related SET domain protein." Proc Natl Acad Sci U S A 98(23): 12902-12907.

Minsky, N., E. Shema, Y. Field, M. Schuster, E. Segal and M. Oren (2008). "Monoubiquitinated $\mathrm{H} 2 \mathrm{~B}$ is associated with the transcribed region of highly expressed genes in human cells." Nat Cell Biol 10(4): 483-488.

Morrison, A. J. and X. Shen (2009). "Chromatin remodelling beyond transcription: the INO80 and SWR1 complexes." Nat Rev Mol Cell Biol 10(6): 373-384.

Muegge, K. (2005). "Lsh, a guardian of heterochromatin at repeat elements." Biochem Cell Biol 83(4): 548-554.

Nagaraj, N. S. and P. K. Datta (2010). "Targeting the transforming growth factor-beta signaling pathway in human cancer." Expert Opin Investig Drugs 19(1): 77-91.

Nakajima, S., R. Doi, E. Toyoda, S. Tsuji, M. Wada, M. Koizumi, S. S. Tulachan, D. Ito, K. Kami, T. Mori, Y. Kawaguchi, K. Fujimoto, R. Hosotani and M. Imamura (2004). "Ncadherin expression and epithelial-mesenchymal transition in pancreatic carcinoma." Clin Cancer Res 10(12 Pt 1): 4125-4133.

Nakajima, Y., T. Yamagishi, S. Hokari and H. Nakamura (2000). "Mechanisms involved in valvuloseptal endocardial cushion formation in early cardiogenesis: roles of transforming growth factor (TGF)-beta and bone morphogenetic protein (BMP)." Anat Rec 258(2): 119-127.

Nakaya, Y., S. Kuroda, Y. T. Katagiri, K. Kaibuchi and Y. Takahashi (2004). "Mesenchymalepithelial transition during somitic segmentation is regulated by differential roles of Cdc42 and Rac1." Dev Cell 7(3): 425-438.

Narayanan, A., W. T. Ruyechan and T. M. Kristie (2007). "The coactivator host cell factor-1 mediates Set1 and MLL1 H3K4 trimethylation at herpesvirus immediate early promoters for initiation of infection." Proc Natl Acad Sci U S A 104(26): 10835-10840.

Narlikar, G. J., H. Y. Fan and R. E. Kingston (2002). "Cooperation between complexes that regulate chromatin structure and transcription." Cell 108(4): 475-487.

Nguyen, L. V., R. Vanner, P. Dirks and C. J. Eaves (2012). "Cancer stem cells: an evolving concept." Nat Rev Cancer 12(2): 133-143. 
Nicassio, F., N. Corrado, J. H. Vissers, L. B. Areces, S. Bergink, J. A. Marteijn, B. Geverts, A. B. Houtsmuller, W. Vermeulen, P. P. Di Fiore and E. Citterio (2007). "Human USP3 is a chromatin modifier required for $S$ phase progression and genome stability." Curr Biol 17(22): 1972-1977.

Nichols, J. T., A. Miyamoto, S. L. Olsen, B. D'Souza, C. Yao and G. Weinmaster (2007). "DSL ligand endocytosis physically dissociates Notch1 heterodimers before activating

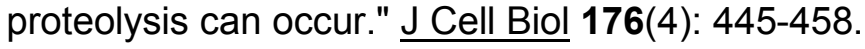

Nicholson, K. M., C. H. Streuli and N. G. Anderson (2003). "Autocrine signalling through erbB receptors promotes constitutive activation of protein kinase B/Akt in breast cancer cell lines." Breast Cancer Res Treat 81(2): 117-128.

Niessen, H. E., J. A. Demmers and J. W. Voncken (2009). "Talking to chromatin: posttranslational modulation of polycomb group function." Epigenetics Chromatin 2(1): 10.

Niessen, K., Y. Fu, L. Chang, P. A. Hoodless, D. McFadden and A. Karsan (2008). "Slug is a direct Notch target required for initiation of cardiac cushion cellularization." $\mathrm{J}$ Cell Biol 182(2): 315-325.

Nieto, M. A. (2013). "Epithelial plasticity: a common theme in embryonic and cancer cells." Science 342(6159): 1234850.

Okitsu, C. Y., J. C. Hsieh and C. L. Hsieh (2010). "Transcriptional activity affects the H3K4me3 level and distribution in the coding region." Mol Cell Biol 30(12): 2933-2946.

Onder, T. T., P. B. Gupta, S. A. Mani, J. Yang, E. S. Lander and R. A. Weinberg (2008). "Loss of E-cadherin promotes metastasis via multiple downstream transcriptional pathways." Cancer Res 68(10): 3645-3654.

Orlando, V. (2003). "Polycomb, epigenomes, and control of cell identity." Cell 112(5): 599606.

Osley, M. A. (2004). "H2B ubiquitylation: the end is in sight." Biochim Biophys Acta 1677(13): 74-78.

Owen-Hughes, T. and M. Bruno (2004). "Molecular biology. Breaking the silence." Science 303(5656): 324-325.

Pandur, P., D. Maurus and M. Kuhl (2002). "Increasingly complex: new players enter the Wnt signaling network." Bioessays 24(10): 881-884.

Pannuti, A., K. Foreman, P. Rizzo, C. Osipo, T. Golde, B. Osborne and L. Miele (2010). "Targeting Notch to target cancer stem cells." Clin Cancer Res 16(12): 3141-3152.

Park, U. H., S. K. Yoon, T. Park, E. J. Kim and S. J. Um (2011). "Additional sex comb-like (ASXL) proteins 1 and 2 play opposite roles in adipogenesis via reciprocal regulation of peroxisome proliferator-activated receptor \{gamma\}." J Biol Chem 286(2): 1354-1363.

Passmore, L. A. and D. Barford (2004). "Getting into position: the catalytic mechanisms of protein ubiquitylation." Biochem J 379(Pt 3): 513-525.

Patel, J. H., Y. Du, P. G. Ard, C. Phillips, B. Carella, C. J. Chen, C. Rakowski, C. Chatterjee, P. M. Lieberman, W. S. Lane, G. A. Blobel and S. B. McMahon (2004). "The C-MYC 
oncoprotein is a substrate of the acetyltransferases hGCN5/PCAF and TIP60." Mol Cell Biol 24(24): 10826-10834.

Peinado, H., D. Olmeda and A. Cano (2007). "Snail, Zeb and bHLH factors in tumour progression: an alliance against the epithelial phenotype?" Nat Rev Cancer 7(6): 415428.

Pena-Llopis, S., S. Vega-Rubin-de-Celis, A. Liao, N. Leng, A. Pavia-Jimenez, S. Wang, T. Yamasaki, L. Zhrebker, S. Sivanand, P. Spence, L. Kinch, T. Hambuch, S. Jain, Y. Lotan, V. Margulis, A. I. Sagalowsky, P. B. Summerour, W. Kabbani, S. W. Wong, N. Grishin, M. Laurent, X. J. Xie, C. D. Haudenschild, M. T. Ross, D. R. Bentley, P. Kapur and J. Brugarolas (2012). "BAP1 loss defines a new class of renal cell carcinoma." Nat Genet 44(7): 751-759.

Pena, C., J. M. Garcia, V. Garcia, J. Silva, G. Dominguez, R. Rodriguez, C. Maximiano, A. Garcia de Herreros, A. Munoz and F. Bonilla (2006). "The expression levels of the transcriptional regulators $\mathrm{p} 300$ and CtBP modulate the correlations between SNAIL, ZEB1, E-cadherin and vitamin D receptor in human colon carcinomas." Int J Cancer 119(9): 2098-2104.

Perla Cota, Mehdi Shafa and Derrick E. Rancourt (2013). Stem Cells and Epigenetic Reprogramming, Pluripotent Stem Cells, Dr. Deepa Bhartiya (Ed.), InTech, DOI: 10.5772/55983. Available from: http://www.intechopen.com/books/pluripotent-stemcells/stem-cells-and-epigenetic-reprogramming

Petruk, S., S. T. Smith, Y. Sedkov and A. Mazo (2008). "Association of trxG and PcG proteins with the bxd maintenance element depends on transcriptional activity." Development 135(14): 2383-2390.

Pickart, C. M. and M. J. Eddins (2004). "Ubiquitin: structures, functions, mechanisms." Biochim Biophys Acta 1695(1-3): 55-72.

Polyak, K. and R. A. Weinberg (2009). "Transitions between epithelial and mesenchymal states: acquisition of malignant and stem cell traits." Nat Rev Cancer 9(4): 265-273.

Ponti, D., A. Costa, N. Zaffaroni, G. Pratesi, G. Petrangolini, D. Coradini, S. Pilotti, M. A. Pierotti and M. G. Daidone (2005). "Isolation and in vitro propagation of tumorigenic breast cancer cells with stem/progenitor cell properties." Cancer Res 65(13): 55065511.

Prenzel, T., Y. Begus-Nahrmann, F. Kramer, M. Hennion, C. Hsu, T. Gorsler, C. Hintermair, D. Eick, E. Kremmer, M. Simons, T. Beissbarth and S. A. Johnsen (2011). "Estrogendependent gene transcription in human breast cancer cells relies upon proteasomedependent monoubiquitination of histone H2B." Cancer Res 71(17): 5739-5753.

Prindull, G. (2005). "Hypothesis: cell plasticity, linking embryonal stem cells to adult stem cell reservoirs and metastatic cancer cells?" Exp Hematol 33(7): 738-746.

Qiao, L., N. Liang, J. Zhang, J. Xie, F. Liu, D. Xu, X. Yu and Y. Tian (2015). "Advanced research on vasculogenic mimicry in cancer." J Cell Mol Med 19(2): 315-326.

Quesada, V., L. Conde, N. Villamor, G. R. Ordonez, P. Jares, L. Bassaganyas, A. J. Ramsay, S. Bea, M. Pinyol, A. Martinez-Trillos, M. Lopez-Guerra, D. Colomer, A. Navarro, T. Baumann, M. Aymerich, M. Rozman, J. Delgado, E. Gine, J. M. Hernandez, 
M. Gonzalez-Diaz, D. A. Puente, G. Velasco, J. M. Freije, J. M. Tubio, R. Royo, J. L. Gelpi, M. Orozco, D. G. Pisano, J. Zamora, M. Vazquez, A. Valencia, H. Himmelbauer, M. Bayes, S. Heath, M. Gut, I. Gut, X. Estivill, A. Lopez-Guillermo, X. S. Puente, E. Campo and C. Lopez-Otin (2012). "Exome sequencing identifies recurrent mutations of the splicing factor SF3B1 gene in chronic lymphocytic leukemia." Nat Genet 44(1): 4752.

Ramadoss, S., X. Chen and C. Y. Wang (2012). "Histone demethylase KDM6B promotes epithelial-mesenchymal transition." J Biol Chem 287(53): 44508-44517.

Rando, O. J. and H. Y. Chang (2009). "Genome-wide views of chromatin structure." Annu Rev Biochem 78: 245-271.

Ranganathan, P., K. L. Weaver and A. J. Capobianco (2011). "Notch signalling in solid tumours: a little bit of everything but not all the time." Nat Rev Cancer 11(5): 338-351.

Reya, T. and H. Clevers (2005). "Wnt signalling in stem cells and cancer." Nature 434(7035): 843-850.

Ricci-Vitiani, L., D. G. Lombardi, E. Pilozzi, M. Biffoni, M. Todaro, C. Peschle and R. De Maria (2007). "Identification and expansion of human colon-cancer-initiating cells." Nature 445(7123): 111-115.

Richards, E. J. and S. C. Elgin (2002). "Epigenetic codes for heterochromatin formation and silencing: rounding up the usual suspects." Cell 108(4): 489-500.

Ringel, M. D., N. Hayre, J. Saito, B. Saunier, F. Schuppert, H. Burch, V. Bernet, K. D. Burman, L. D. Kohn and M. Saji (2001). "Overexpression and overactivation of Akt in thyroid carcinoma." Cancer Res 61(16): 6105-6111.

Robinson, J. T., H. Thorvaldsdottir, W. Winckler, M. Guttman, E. S. Lander, G. Getz and J. P. Mesirov (2011). "Integrative genomics viewer." Nat Biotechnol 29(1): 24-26.

Ropero, S. and M. Esteller (2007). "The role of histone deacetylases (HDACs) in human cancer." Mol Oncol 1(1): 19-25.

Sadowski, M. and B. Sarcevic (2010). "Mechanisms of mono- and poly-ubiquitination: Ubiquitination specificity depends on compatibility between the E2 catalytic core and amino acid residues proximal to the lysine." Cell Div 5: 19.

Sahtoe, D. D., W. J. van Dijk, R. Ekkebus, H. Ovaa and T. K. Sixma (2016). "BAP1/ASXL1 recruitment and activation for H2A deubiquitination." Nat Commun 7: 10292.

Samanta, D. and P. K. Datta (2012). "Alterations in the Smad pathway in human cancers." Front Biosci (Landmark Ed) 17: 1281-1293.

Sampieri, K. and R. Fodde (2012). "Cancer stem cells and metastasis." Semin Cancer Biol 22(3): 187-193.

Sanchez-Pulido, L., L. Kong and C. P. Ponting (2012). "A common ancestry for BAP1 and Uch37 regulators." Bioinformatics 28(15): 1953-1956.

Sanchez, R. and M. M. Zhou (2011). "The PHD finger: a versatile epigenome reader." Trends Biochem Sci 36(7): 364-372. 
Sandoval, J. and M. Esteller (2012). "Cancer epigenomics: beyond genomics." Curr Opin Genet Dev 22(1): 50-55.

Scheuermann, J. C., A. G. de Ayala Alonso, K. Oktaba, N. Ly-Hartig, R. K. McGinty, S. Fraterman, M. Wilm, T. W. Muir and J. Muller (2010). "Histone H2A deubiquitinase activity of the Polycomb repressive complex PR-DUB." Nature 465(7295): 243-247.

Scott, M. T., K. Korfi, P. Saffrey, L. E. Hopcroft, R. Kinstrie, F. Pellicano, C. Guenther, P. Gallipoli, M. Cruz, K. Dunn, H. G. Jorgensen, J. E. Cassels, A. Hamilton, A. Crossan, A. Sinclair, T. L. Holyoake and D. Vetrie (2016). "Epigenetic Reprogramming Sensitizes CML Stem Cells to Combined EZH2 and Tyrosine Kinase Inhibition." Cancer Discov.

Scotto, L., G. Narayan, S. V. Nandula, H. Arias-Pulido, S. Subramaniyam, A. Schneider, A. M. Kaufmann, J. D. Wright, B. Pothuri, M. Mansukhani and V. V. Murty (2008). "Identification of copy number gain and overexpressed genes on chromosome arm 20q by an integrative genomic approach in cervical cancer: potential role in progression." Genes Chromosomes Cancer 47(9): 755-765.

Seligson, D. B., S. Horvath, M. A. McBrian, V. Mah, H. Yu, S. Tze, Q. Wang, D. Chia, L. Goodglick and S. K. Kurdistani (2009). "Global levels of histone modifications predict prognosis in different cancers." Am J Pathol 174(5): 1619-1628.

Serrano-Gomez, S. J., M. Maziveyi and S. K. Alahari (2016). "Regulation of epithelialmesenchymal transition through epigenetic and post-translational modifications." Mol Cancer 15: 18.

Sharma, S., T. K. Kelly and P. A. Jones (2010). "Epigenetics in cancer." $\underline{\text { Carcinogenesis }}$ 31(1): 27-36.

Shema, E., I. Tirosh, Y. Aylon, J. Huang, C. Ye, N. Moskovits, N. Raver-Shapira, N. Minsky, J. Pirngruber, G. Tarcic, P. Hublarova, L. Moyal, M. Gana-Weisz, Y. Shiloh, Y. Yarden, S. A. Johnsen, B. Vojtesek, S. L. Berger and M. Oren (2008). "The histone H2B-specific ubiquitin ligase RNF20/hBRE1 acts as a putative tumor suppressor through selective regulation of gene expression." Genes Dev 22(19): 2664-2676.

Shen, C., Y. Wang, P. Wei and X. Du (2016). "BRCA1-associated protein 1 deficiency in lung adenocarcinoma predicts poor outcome and increased tumor invasion." BMC Cancer 16(1): 670.

Shi, Y., F. Lan, C. Matson, P. Mulligan, J. R. Whetstine, P. A. Cole, R. A. Casero and Y. Shi (2004). "Histone demethylation mediated by the nuclear amine oxidase homolog LSD1." Cell 119(7): 941-953.

Shi, Y. and J. Massague (2003). "Mechanisms of TGF-beta signaling from cell membrane to the nucleus." Cell 113(6): 685-700.

Shih, A. H., O. Abdel-Wahab, J. P. Patel and R. L. Levine (2012). "The role of mutations in epigenetic regulators in myeloid malignancies." Nat Rev Cancer 12(9): 599-612.

Shin, H., T. Liu, A. K. Manrai and X. S. Liu (2009). "CEAS: cis-regulatory element annotation system." Bioinformatics 25(19): 2605-2606.

Shukla, A., N. Stanojevic, Z. Duan, T. Shadle and S. R. Bhaumik (2006). "Functional analysis of H2B-Lys-123 ubiquitination in regulation of H3-Lys-4 methylation and 
recruitment of RNA polymerase II at the coding sequences of several active genes in vivo." J Biol Chem 281(28): 19045-19054.

Sinclair, D. A., T. A. Milne, J. W. Hodgson, J. Shellard, C. A. Salinas, M. Kyba, F. Randazzo and H. W. Brock (1998). "The Additional sex combs gene of Drosophila encodes a chromatin protein that binds to shared and unique Polycomb group sites on polytene chromosomes." Development 125(7): 1207-1216.

Song, L. B., J. Li, W. T. Liao, Y. Feng, C. P. Yu, L. J. Hu, Q. L. Kong, L. H. Xu, X. Zhang, W. L. Liu, M. Z. Li, L. Zhang, T. B. Kang, L. W. Fu, W. L. Huang, Y. F. Xia, S. W. Tsao, M. $\mathrm{Li}$, V. Band, H. Band, Q. H. Shi, Y. X. Zeng and M. S. Zeng (2009). "The polycomb group protein Bmi-1 represses the tumor suppressor PTEN and induces epithelialmesenchymal transition in human nasopharyngeal epithelial cells." $\underline{\mathrm{J} C l i n}$ Invest 119(12): 3626-3636.

Strahl, B. D. and C. D. Allis (2000). "The language of covalent histone modifications." Nature 403(6765): 41-45.

Stransky, N., A. M. Egloff, A. D. Tward, A. D. Kostic, K. Cibulskis, A. Sivachenko, G. V. Kryukov, M. S. Lawrence, C. Sougnez, A. McKenna, E. Shefler, A. H. Ramos, P. Stojanov, S. L. Carter, D. Voet, M. L. Cortes, D. Auclair, M. F. Berger, G. Saksena, C. Guiducci, R. C. Onofrio, M. Parkin, M. Romkes, J. L. Weissfeld, R. R. Seethala, L. Wang, C. Rangel-Escareno, J. C. Fernandez-Lopez, A. Hidalgo-Miranda, J. MelendezZajgla, W. Winckler, K. Ardlie, S. B. Gabriel, M. Meyerson, E. S. Lander, G. Getz, T. R. Golub, L. A. Garraway and J. R. Grandis (2011). "The mutational landscape of head and neck squamous cell carcinoma." Science 333(6046): 1157-1160.

Subramanian, A., P. Tamayo, V. K. Mootha, S. Mukherjee, B. L. Ebert, M. A. Gillette, A. Paulovich, S. L. Pomeroy, T. R. Golub, E. S. Lander and J. P. Mesirov (2005). "Gene set enrichment analysis: a knowledge-based approach for interpreting genome-wide expression profiles." Proc Natl Acad Sci U S A 102(43): 15545-15550.

Suganuma, T. and J. L. Workman (2011). "Signals and combinatorial functions of histone modifications." Annu Rev Biochem 80: 473-499.

Sugimoto, Y., H. Muramatsu, H. Makishima, C. Prince, A. M. Jankowska, N. Yoshida, Y. Xu, N. Nishio, A. Hama, H. Yagasaki, Y. Takahashi, K. Kato, A. Manabe, S. Kojima and J. P. Maciejewski (2010). "Spectrum of molecular defects in juvenile myelomonocytic leukaemia includes ASXL1 mutations." Br J Haematol 150(1): 83-87.

Sun, T., N. Zhao, X. L. Zhao, Q. Gu, S. W. Zhang, N. Che, X. H. Wang, J. Du, Y. X. Liu and B. C. Sun (2010). "Expression and functional significance of Twist1 in hepatocellular carcinoma: its role in vasculogenic mimicry." Hepatology 51(2): 545-556.

Takai, D. and P. A. Jones (2002). "Comprehensive analysis of CpG islands in human chromosomes 21 and 22." Proc Natl Acad Sci U S A 99(6): 3740-3745.

Talbert, P. B. and S. Henikoff (2006). "Spreading of silent chromatin: inaction at a distance." Nat Rev Genet 7(10): 793-803.

Tam, W. L. and R. A. Weinberg (2013). "The epigenetics of epithelial-mesenchymal plasticity in cancer." Nat Med 19(11): 1438-1449. 
Taverna, S. D., H. Li, A. J. Ruthenburg, C. D. Allis and D. J. Patel (2007). "How chromatinbinding modules interpret histone modifications: lessons from professional pocket pickers." Nat Struct Mol Biol 14(11): 1025-1040.

ten Berge, D., W. Koole, C. Fuerer, M. Fish, E. Eroglu and R. Nusse (2008). "Wnt signaling mediates self-organization and axis formation in embryoid bodies." Cell Stem Cell 3(5): 508-518.

Testa, J. R. and A. Bellacosa (2001). "AKT plays a central role in tumorigenesis." Proc Natl Acad Sci U S A 98(20): 10983-10985.

Thiery, J. P., H. Acloque, R. Y. Huang and M. A. Nieto (2009). "Epithelial-mesenchymal transitions in development and disease." Cell 139(5): 871-890.

Thiery, J. P. and J. P. Sleeman (2006). "Complex networks orchestrate epithelialmesenchymal transitions." Nat Rev Mol Cell Biol 7(2): 131-142.

Thomas, J. O. (1999). "Histone H1: location and role." Curr Opin Cell Biol 11(3): 312-317.

Thornton, J. L., G. H. Westfield, Y. H. Takahashi, M. Cook, X. Gao, A. R. Woodfin, J. S. Lee, M. A. Morgan, J. Jackson, E. R. Smith, J. F. Couture, G. Skiniotis and A. Shilatifard (2014). "Context dependency of Set1/COMPASS-mediated histone H3 Lys4 trimethylation." Genes Dev 28(2): 115-120.

Timmerman, L. A., J. Grego-Bessa, A. Raya, E. Bertran, J. M. Perez-Pomares, J. Diez, S. Aranda, S. Palomo, F. McCormick, J. C. Izpisua-Belmonte and J. L. de la Pompa (2004). "Notch promotes epithelial-mesenchymal transition during cardiac development and oncogenic transformation." Genes Dev 18(1): 99-115.

Towbin, H., T. Staehelin and J. Gordon (1992). "Electrophoretic transfer of proteins from polyacrylamide gels to nitrocellulose sheets: procedure and some applications. 1979." Biotechnology 24: 145-149.

Tropberger, P. and R. Schneider (2010). "Going global: novel histone modifications in the globular domain of H3." Epigenetics 5(2): 112-117.

Tsai, J. H. and J. Yang (2013). "Epithelial-mesenchymal plasticity in carcinoma metastasis." Genes Dev 27(20): 2192-2206.

Turner, B. M. (1993). "Decoding the nucleosome." Cell 75(1): 5-8.

Tyagi, S., A. L. Chabes, J. Wysocka and W. Herr (2007). "E2F activation of S phase promoters via association with HCF-1 and the MLL family of histone H3K4 methyltransferases." Mol Cell 27(1): 107-119.

Valletta, S., H. Dolatshad, M. Bartenstein, B. H. Yip, E. Bello, S. Gordon, Y. Yu, J. Shaw, S. Roy, L. Scifo, A. Schuh, A. Pellagatti, T. A. Fulga, A. Verma and J. Boultwood (2015). "ASXL1 mutation correction by CRISPR/Cas9 restores gene function in leukemia cells and increases survival in mouse xenografts." Oncotarget 6(42): 44061-44071.

Van de Walle, I., G. De Smet, M. Gartner, M. De Smedt, E. Waegemans, B. Vandekerckhove, G. Leclercq, J. Plum, J. C. Aster, I. D. Bernstein, C. J. Guidos, B. Kyewski and T. Taghon (2011). "Jagged2 acts as a Delta-like Notch ligand during early hematopoietic cell fate decisions." Blood 117(17): 4449-4459. 
Vempati, R. K., R. S. Jayani, D. Notani, A. Sengupta, S. Galande and D. Haldar (2010). "p300-mediated acetylation of histone $\mathrm{H} 3$ lysine 56 functions in DNA damage response in mammals." J Biol Chem 285(37): 28553-28564.

Vicente-Duenas, C., J. Gutierrez de Diego, F. D. Rodriguez, R. Jimenez and C. Cobaleda (2009). "The role of cellular plasticity in cancer development." Curr Med Chem 16(28): 3676-3685.

Visvader, J. E. and G. J. Lindeman (2008). "Cancer stem cells in solid tumours: accumulating evidence and unresolved questions." Nat Rev Cancer 8(10): 755-768.

Vogelstein, B. and K. W. Kinzler (2004). "Cancer genes and the pathways they control." Nat Med 10(8): 789-799.

Volinia, S., G. A. Calin, C. G. Liu, S. Ambs, A. Cimmino, F. Petrocca, R. Visone, M. Iorio, C. Roldo, M. Ferracin, R. L. Prueitt, N. Yanaihara, G. Lanza, A. Scarpa, A. Vecchione, M. Negrini, C. C. Harris and C. M. Croce (2006). "A microRNA expression signature of human solid tumors defines cancer gene targets." Proc Natl Acad Sci U S A 103(7): 2257-2261.

Wang, G. G., C. D. Allis and P. Chi (2007). "Chromatin remodeling and cancer, Part I: Covalent histone modifications." Trends Mol Med 13(9): 363-372.

Wang, H., S. Y. Quah, J. M. Dong, E. Manser, J. P. Tang and Q. Zeng (2007). "PRL-3 downregulates PTEN expression and signals through PI3K to promote epithelialmesenchymal transition." Cancer Res 67(7): 2922-2926.

Wang, H., L. Wang, H. Erdjument-Bromage, M. Vidal, P. Tempst, R. S. Jones and Y. Zhang (2004). "Role of histone H2A ubiquitination in Polycomb silencing." Nature 431(7010): 873-878.

Wang, J., Z. Li, Y. He, F. Pan, S. Chen, S. Rhodes, L. Nguyen, J. Yuan, L. Jiang, X. Yang, O. Weeks, Z. Liu, J. Zhou, H. Ni, C. L. Cai, M. Xu and F. C. Yang (2014). "Loss of Asxl1 leads to myelodysplastic syndrome-like disease in mice." Blood 123(4): 541-553.

Wang, Y. and F. C. Leung (2004). "An evaluation of new criteria for CpG islands in the human genome as gene markers." Bioinformatics 20(7): 1170-1177.

Wang, Y. and Y. Shang (2013). "Epigenetic control of epithelial-to-mesenchymal transition and cancer metastasis." Exp Cell Res 319(2): 160-169.

Wang, Y. and B. P. Zhou (2013). "Epithelial-mesenchymal Transition---A Hallmark of Breast Cancer Metastasis." Cancer Hallm 1(1): 38-49.

Wang, Z., S. Banerjee, Y. Li, K. M. Rahman, Y. Zhang and F. H. Sarkar (2006). "Downregulation of notch-1 inhibits invasion by inactivation of nuclear factor-kappaB, vascular endothelial growth factor, and matrix metalloproteinase- 9 in pancreatic cancer cells." Cancer Res 66(5): 2778-2784.

Wang, Z., Y. Zhang, S. Banerjee, Y. Li and F. H. Sarkar (2006). "Inhibition of nuclear factor kappab activity by genistein is mediated via Notch-1 signaling pathway in pancreatic cancer cells." Int J Cancer 118(8): 1930-1936. 
Wellner, U., J. Schubert, U. C. Burk, O. Schmalhofer, F. Zhu, A. Sonntag, B. Waldvogel, C. Vannier, D. Darling, A. zur Hausen, V. G. Brunton, J. Morton, O. Sansom, J. Schuler, M. P. Stemmler, C. Herzberger, U. Hopt, T. Keck, S. Brabletz and T. Brabletz (2009). "The EMT-activator ZEB1 promotes tumorigenicity by repressing stemness-inhibiting microRNAs." Nat Cell Biol 11(12): 1487-1495.

Wheelock, M. J., Y. Shintani, M. Maeda, Y. Fukumoto and K. R. Johnson (2008). "Cadherin switching." J Cell Sci 121(Pt 6): 727-735.

Widschwendter, M. and P. A. Jones (2002). "DNA methylation and breast carcinogenesis." Oncogene 21(35): 5462-5482.

Williams, D. S., M. J. Bird, R. N. Jorissen, Y. L. Yu, F. Walker, H. H. Zhang, E. C. Nice and A. W. Burgess (2010). "Nonsense mediated decay resistant mutations are a source of expressed mutant proteins in colon cancer cell lines with microsatellite instability." PLoS One 5(12): e16012.

Wilson, B. G. and C. W. Roberts (2011). "SWI/SNF nucleosome remodellers and cancer." Nat Rev Cancer 11(7): 481-492.

Wood, A., J. Schneider and A. Shilatifard (2005). "Cross-talking histones: implications for the regulation of gene expression and DNA repair." Biochem Cell Biol 83(4): 460-467.

Wood, L. D., D. W. Parsons, S. Jones, J. Lin, T. Sjoblom, R. J. Leary, D. Shen, S. M. Boca, T. Barber, J. Ptak, N. Silliman, S. Szabo, Z. Dezso, V. Ustyanksky, T. Nikolskaya, Y. Nikolsky, R. Karchin, P. A. Wilson, J. S. Kaminker, Z. Zhang, R. Croshaw, J. Willis, D. Dawson, M. Shipitsin, J. K. Willson, S. Sukumar, K. Polyak, B. H. Park, C. L. Pethiyagoda, P. V. Pant, D. G. Ballinger, A. B. Sparks, J. Hartigan, D. R. Smith, E. Suh, N. Papadopoulos, P. Buckhaults, S. D. Markowitz, G. Parmigiani, K. W. Kinzler, V. E. Velculescu and B. Vogelstein (2007). "The genomic landscapes of human breast and colorectal cancers." Science 318(5853): 1108-1113.

Wu, M., P. F. Wang, J. S. Lee, S. Martin-Brown, L. Florens, M. Washburn and A. Shilatifard (2008). "Molecular regulation of H3K4 trimethylation by Wdr82, a component of human Set1/COMPASS." Mol Cell Biol 28(24): 7337-7344.

Wu, M. Z., Y. P. Tsai, M. H. Yang, C. H. Huang, S. Y. Chang, C. C. Chang, S. C. Teng and K. J. Wu (2011). "Interplay between HDAC3 and WDR5 is essential for hypoxia-induced epithelial-mesenchymal transition." Mol Cell 43(5): 811-822.

Wu, X., I. H. Bekker-Jensen, J. Christensen, K. D. Rasmussen, S. Sidoli, Y. Qi, Y. Kong, X. Wang, Y. Cui, Z. Xiao, G. Xu, K. Williams, J. Rappsilber, C. K. Sonderby, O. Winther, O. N. Jensen and K. Helin (2015). "Tumor suppressor ASXL1 is essential for the activation of INK4B expression in response to oncogene activity and anti-proliferative signals." Cell Res 25(11): 1205-1218.

Xiao, T., C. F. Kao, N. J. Krogan, Z. W. Sun, J. F. Greenblatt, M. A. Osley and B. D. Strahl (2005). "Histone H2B ubiquitylation is associated with elongating RNA polymerase II." Mol Cell Biol 25(2): 637-651.

Yang-Snyder, J., J. R. Miller, J. D. Brown, C. J. Lai and R. T. Moon (1996). "A frizzled homolog functions in a vertebrate Wnt signaling pathway." Curr Biol 6(10): 1302-1306. 
Yang, M. H., C. L. Chen, G. Y. Chau, S. H. Chiou, C. W. Su, T. Y. Chou, W. L. Peng and J. C. Wu (2009). "Comprehensive analysis of the independent effect of twist and snail in promoting metastasis of hepatocellular carcinoma." Hepatology 50(5): 1464-1474.

Yang, M. H., D. S. Hsu, H. W. Wang, H. J. Wang, H. Y. Lan, W. H. Yang, C. H. Huang, S. Y. Kao, C. H. Tzeng, S. K. Tai, S. Y. Chang, O. K. Lee and K. J. Wu (2010). "Bmi1 is essential in Twist1-induced epithelial-mesenchymal transition." Nat Cell Biol 12(10): 982-992.

Yang, X. J. (2005). "Multisite protein modification and intramolecular signaling." Oncogene 24(10): 1653-1662.

Yang, X. J. and E. Seto (2007). "HATs and HDACs: from structure, function and regulation to novel strategies for therapy and prevention." Oncogene 26(37): 5310-5318.

Yao, X. H., Y. F. Ping and X. W. Bian (2011). "Contribution of cancer stem cells to tumor vasculogenic mimicry." Protein Cell 2(4): 266-272.

Yasui, W., N. Oue, S. Ono, Y. Mitani, R. Ito and H. Nakayama (2003). "Histone acetylation and gastrointestinal carcinogenesis." Ann N Y Acad Sci 983: 220-231.

Yilmaz, M. and G. Christofori (2009). "EMT, the cytoskeleton, and cancer cell invasion." Cancer Metastasis Rev 28(1-2): 15-33.

Yoshikawa, M., K. Hishikawa, T. Marumo and T. Fujita (2007). "Inhibition of histone deacetylase activity suppresses epithelial-to-mesenchymal transition induced by TGFbeta1 in human renal epithelial cells." J Am Soc Nephrol 18(1): 58-65.

Zajicek, G. (1996). "A new cancer hypothesis." Med Hypotheses 47(2): 111-115.

Zavadil, J. and E. P. Bottinger (2005). "TGF-beta and epithelial-to-mesenchymal transitions." Oncogene 24(37): 5764-5774.

Zhang, F. F., R. Cardarelli, J. Carroll, S. Zhang, K. G. Fulda, K. Gonzalez, J. K. Vishwanatha, A. Morabia and R. M. Santella (2011). "Physical activity and global genomic DNA methylation in a cancer-free population." Epigenetics 6(3): 293-299.

Zhang, H. Y., F. Liang, Z. L. Jia, S. T. Song and Z. F. Jiang (2013). "PTEN mutation, methylation and expression in breast cancer patients." Oncol Lett 6(1): 161-168.

Zhang, P., C. Xing, S. D. Rhodes, Y. He, K. Deng, Z. Li, F. He, C. Zhu, L. Nguyen, Y. Zhou, S. Chen, K. S. Mohammad, T. A. Guise, O. Abdel-Wahab, M. Xu, Q. F. Wang and F. C. Yang (2016). "Loss of Asxl1 Alters Self-Renewal and Cell Fate of Bone Marrow Stromal Cell, Leading to Bohring-Opitz-like Syndrome in Mice." Stem Cell Reports 6(6): 914925.

Zhang, S., C. Balch, M. W. Chan, H. C. Lai, D. Matei, J. M. Schilder, P. S. Yan, T. H. Huang and K. P. Nephew (2008). "Identification and characterization of ovarian cancerinitiating cells from primary human tumors." Cancer Res 68(11): 4311-4320.

Zhang, X. Y., M. Varthi, S. M. Sykes, C. Phillips, C. Warzecha, W. Zhu, A. Wyce, A. W. Thorne, S. L. Berger and S. B. McMahon (2008). "The putative cancer stem cell marker USP22 is a subunit of the human SAGA complex required for activated transcription and cell-cycle progression." Mol Cell 29(1): 102-111. 
Zhang, Y., T. Liu, C. A. Meyer, J. Eeckhoute, D. S. Johnson, B. E. Bernstein, C. Nusbaum, R. M. Myers, M. Brown, W. Li and X. S. Liu (2008). "Model-based analysis of ChIP-Seq (MACS)." Genome Biol 9(9): R137.

Zhao, L., W. Li, W. Zang, Z. Liu, X. Xu, H. Yu, Q. Yang and J. Jia (2013). "JMJD2B promotes epithelial-mesenchymal transition by cooperating with beta-catenin and enhances gastric cancer metastasis." Clin Cancer Res 19(23): 6419-6429.

Zhu, B., Y. Zheng, A. D. Pham, S. S. Mandal, H. Erdjument-Bromage, P. Tempst and D. Reinberg (2005). "Monoubiquitination of human histone H2B: the factors involved and their roles in HOX gene regulation." Mol Cell 20(4): 601-611. 
